DEVELOPMENT OF AN ADVANCED 2D THGEM MICRODOSIMETRIC DETECTOR 


\title{
DEVELOPMENT OF AN ADVANCED TWO-DIMENSIONAL MICRODOSIMETRIC DETECTOR BASED ON THICK GAS ELECTRON MULTIPLIERS
}

\author{
By \\ SAHAR DARVISH-MOLLA, B.Sc, M.Sc
}

\author{
A Thesis \\ Submitted to the School of Graduate Studies \\ in Partial Fulfilment of the Requirements \\ for the Degree \\ Doctor of Philosophy
}

McMaster University

(C) Copyright by Sahar Darvish-Molla, 2016 
DOCTOR OF PHILOSOPHY (2016)

Medical Physics
McMaster University

Hamilton, Ontario

TITLE: Development of an Advanced Two-Dimensional Microdosimetric Detector based on THick Gas Electron Multipliers
AUTHOR:
Sahar Darvish-Molla, B.Sc
M.Sc
SUPERVISOR:
Dr. Soo Hyun Byun
NUMBER OF PAGES: $\quad \mathrm{xx}, 187$ 


\section{Abstract}

The THick Gas Electron Multiplier (THGEM) based tissue-equivalent proportional counter (TEPC) has been proven to be useful for microdosimetry due to its flexibility in varying the gaseous sensitive volume and achieving high multiplication gain. Aiming at measuring the spatial distribution of radiation dose for mixed neutron-gamma fields, an advanced two-dimensional (2D) THGEM TEPC was designed and constructed at McMaster University which will enable us to overcome the operational limitation of the classical TEPCs, particularly for high dose rate fields. Compared to the traditional TEPCs, anode wire electrodes were replaced by THGEM layer, which not only enhances the gas multiplication gain but also offers a flexible and convenient fabrication or building 2D detectors.

The 2D THGEM TEPC consists of an array of $3 \times 3$ sensitive volumes, equivalent to 9 individual TEPCs, each of which has a dimension of $5 \mathrm{~mm}$ diameter and length. Taking the overall cost, size and flexibility into account, to process 9 detectors signals simultaneously, a multi-input digital pulse processing system was developed by using modern microcontrollers, each of which is coupled to a 12-bit sampling ADC with a sampling rate of $42 \mathrm{Msps}$. The signal processing system was tested using a $\mathrm{NaI}(\mathrm{Tl})$ detector, which has proven that is it faster than a traditional analogue system and a commercial digital system. Using the McMaster Tandetron ${ }^{7} \operatorname{Li}(\mathrm{p}, \mathrm{n})$ accelerator neutron source, both fundamental detector performance, as well as neutron dosimetric response of the 2D THGEM TEPC, has been extensively investigated and compared to the data acquired by a spherical TEPC. It was shown that the microdosimetric response and the measured absorbed dose rate of the 2D THGEM detector developed in this study are comparable to the standard 1/2" TEPC which is commercially available. 


\section{Acknowledgements}

I owe a debt of gratitude to a number of people who assisted me materially and spiritually during four years of studying, researching and writing my $\mathrm{PhD}$ thesis.

First among equals, is Dr. Soo Hyun Byun, my experienced caring supervisor who has supported me during these four years with his immense knowledge, patience and motivation. His positive outlooks and encouragements in my research inspired me and gave me confidence. Thank you for believing in me and giving me a unique opportunity to work on such an important interesting topic. Thank you for your precious empathy and guidance during the numerous moments of frustration that I experienced all through my research.

Dr. William Prestwich, Dr. David Chettle, Dr. Anthony Waker and Jovica Atanakovic, my enlightened committee supervisors, for offering fruitful discussions and invaluable advice during the course of my research. Kenrick Chin, an astute, gracious and proficient man, for his hard work for designing and constructing the multi-input digital signal processing system, without which, processing of 9 detectors signals would have been extremely expensive and not really possible. Thank you for your great work Kenrick!

Fiona Ahlang, Linda Ellis, Nancy Brand and Wendy Malarek, the medical physics department staff, for their motherly support during the past six years of being at McMaster University. Justin Bennett, for running the Tandetron accelerator, allowing me access to different tools and equipment that I was desperately looking for. Justin, Thank you for all your efforts to keep up with my measurements and long day accelerator runs, even during your precious lunch times! Dr. Andrei Hanu, whose experience in developing a THGEM detector was a great help to me. Mr. Yousef Shidfar, my great friend, for all his technical supports at the very beginning of my research. All my friends, for keeping me company and for being there for me. Specially, Niloufar and Iman, two of the best loyal and warmhearted friends, who have been there for me in all my hard and disappointed times. Thank you for making me laugh loud and thank you for all your wonderful prayers and good wishes. 
Vasy and Jim Daniolos my beloved people for their great support and compassion. Thank you for accepting me in your home and heart. Because of you and your unconditional love and support, I never felt lonely. Spiro, Kevin and Angie my Canadian brothers and sister. Thank you for keep saying that I am smart! Thank you all for being my Canadian family.

My only brother, Saman, for his supportive gracious spirit. Thank you for being with me in my hard parts of life, which indeed without you and your accompaniment and compassion it would not be possible to leave them all behind.

I am truly grateful to my lovely caring parents: to my mother, Soheila, whose continuous uncountable sacrifices, encouragements and supports were an unremitting source of inspiration for me. Being away from her is a real torture in my life, but thank you for being virtually with me through internet early mornings and late nights. You gave up the most important things in your life so that I could take on the most important things in mine. Love you mom! To my father, Bijan, who I don't know if I would be able to thank him for any words, for his true love and advice. Thank you for putting hard on yourself to make my life comfortable. Thank you for all you gave me materially and spiritually. You always called me Dr. Sahar, since I started high school, and now, I want to thank you and all your sacrifices, by becoming a real Dr. Sahar!

To my amiable husband, my first and last love, Fariborz, for his unquestioning support, patience and love. Thank you for your countless trust, positive attitude and soothing words in all my frustrating moments in life. You taught me there is more to the life than what I feel. I appreciate your big picture and how you deal with my detailed view. Thank you so much!

During years of my research, I have learned that there is always a solution to issues that confront you in your project, no matter how hard or beyond your skills they are, there is no room for disappointment and you have to conquer each challenge step by step. Thank you, my God, for everything you have made to happen in my life and for always leading me towards my best. Thank you for giving me the chance and strength to make mine and my parent's dream come true. 


\section{Table of Contents}

Abstract

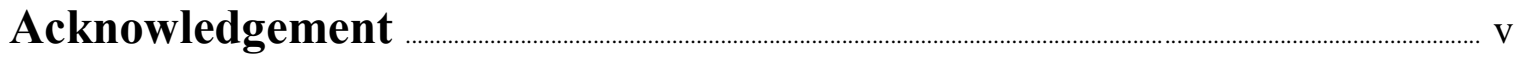

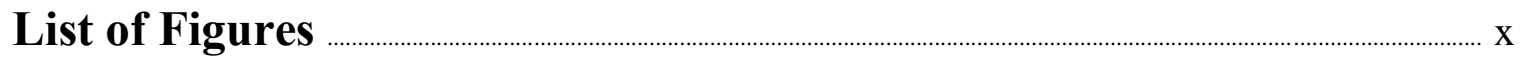

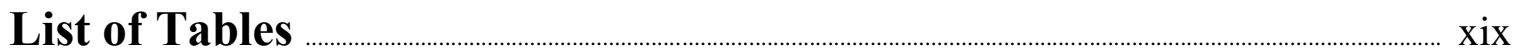

Chapter 1. Introduction $\ldots$

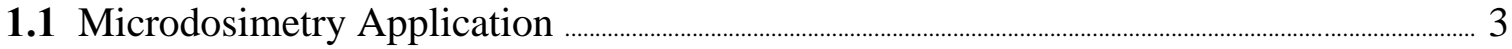

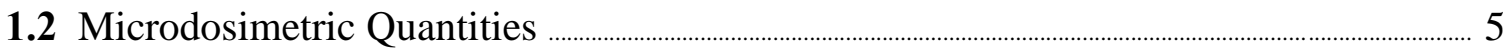

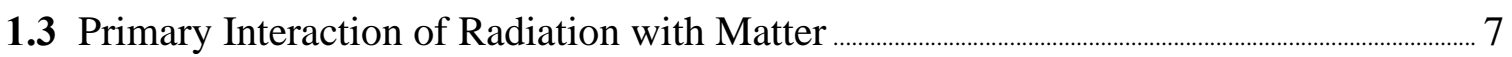

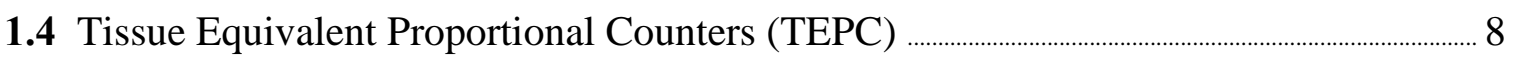

1.4.1 Traditional Generation of TEPCs ……........................................................... 9

1.4.2 New Generation of TEPCs ............................................................... 10

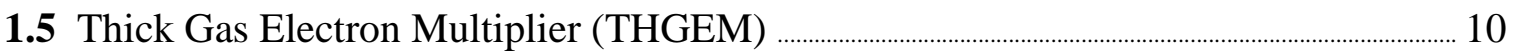

1.6 Motivation, Goal and Approach of the Thesis …….............................................................. 14

Chapter 2. Detector Design and Construction ………… 16

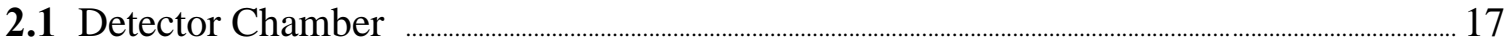

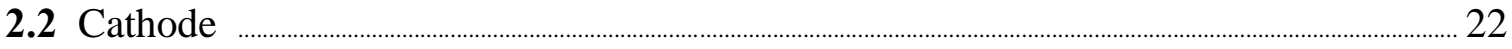

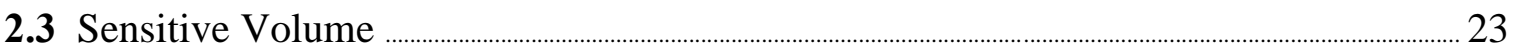

2.4 THick Gas Electron Multiplier (THGEM) …_................................................. 24

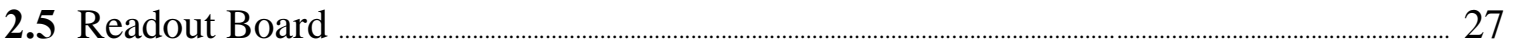

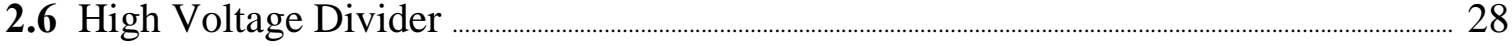

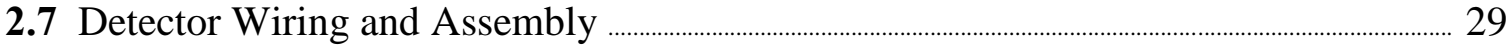




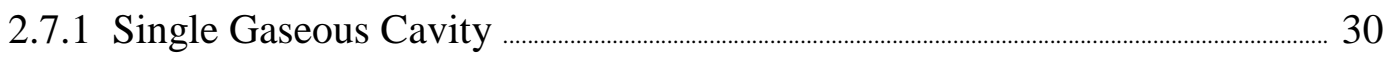

2.7.2 2D Multi-volume (Array of $3 \times 3$ Gaseous Cavities) …................................... 31

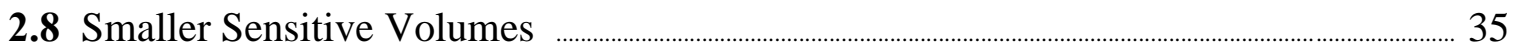

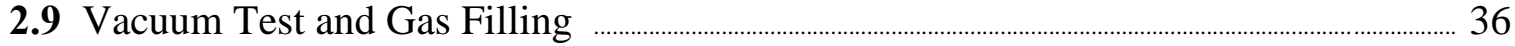

Chapter 3. Development of McMaster Multi-Input Digital Signal

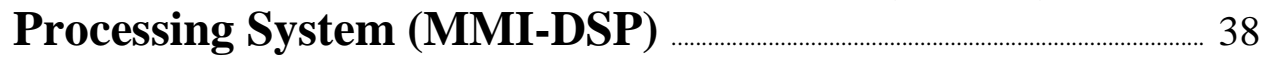

3.1 Design and Fabrication of McMaster Prototype Single-Input DSP (MSI-DSP) ......... 39

3.2 Design Extension to the McMaster Multi-Input DSP (MMI-DSP) ………………….. 53

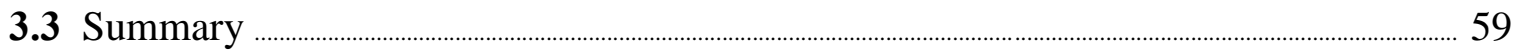

Chapter 4. Experimental Methodology …__ _ 60

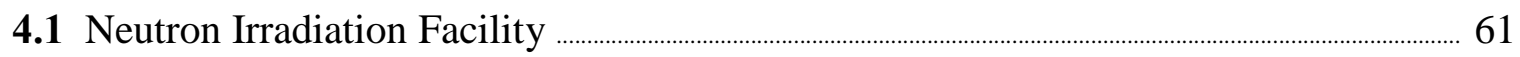

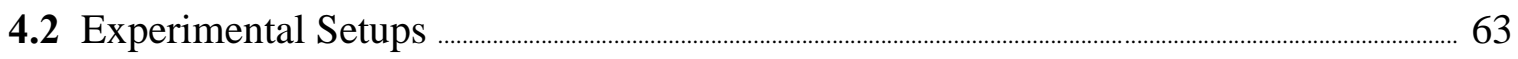

4.2.1 Single gaseous cavity: Preliminary neutron measurements with commercial DSP ……

4.2.2 Single gaseous cavity: Preliminary neutron measurements with prototype MSI-DSP

4.2.3 $3 \times 3$ cavity array: Neutron measurements with MMI-DSP

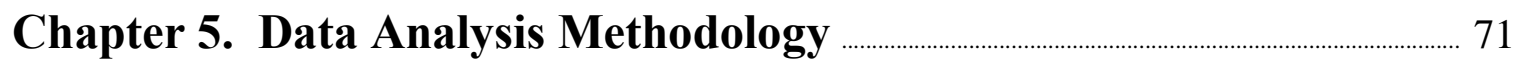

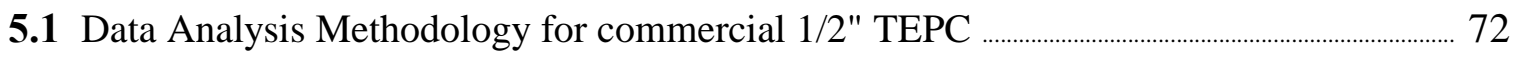

5.2 Data Analysis Methodology for 2D THGEM Detector …..................................................

Chapter 6. Comprehensive Dose Measurement for the ${ }^{7} \mathrm{Li}(\mathbf{p}, \mathbf{n})$

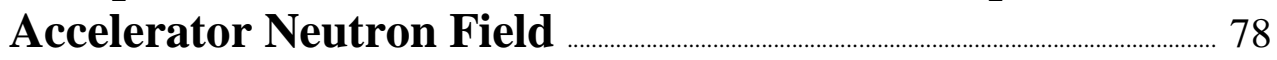

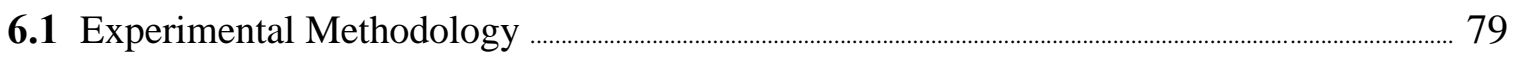

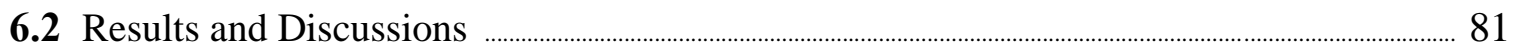


6.3 Conclusion

Chapter 7. Results and Discussions 100

7.1 Single Gaseous Cavity Detector 103

7.1.1 Signal Performance and Multiplication Gain 105

7.1.2 Stability and Reproducibility 107

7.1.3 Microdosimetric Spectrum 115

7.1.4 Chemically Cleaned THGEM Data 120

7.1.5 Performance Test Results using prototype MSI-DSP vs. Commercial DSP 122

7.1.6 2k Data vs. 16k Data 124

7.1.7 Summary 127

7.2 2D Multi-Volume Detector $(3 \times 3$ gaseous Cavity Array) 128

7.2.1 2D Single-Layer THGEM Detector Performance 129

7.2.2 2D Double-Layer THGEM Detector Performance 162

7.3 Absorbed Dose Distribution 169

7.4 2D THGEM Detector with Smaller Size Sensitive Volume 172

Chapter 8. Future Works and Conclusions 177

8.1 Encountered Challenges and Proposed Future Works 178

8.2 Thesis Conclusion 181 


\section{List of Figures}

Fig. 1.1 a. Electron microscope picture of a section of typical GEM electrode, $50 \mu \mathrm{m}$ thick. The holes pitch and diameter are 140 and $70 \mathrm{~mm}$, respectively, b. Electric field in the region of the holes of a GEM electrode

Fig. 1.2 Simulated electron avalanche process for a THGEM with $0.9 \mathrm{~mm}$ thickness and $0.6 \mathrm{~mm}$ hole diameter and $1200 \mathrm{~V}$ bias voltage

Fig. 1.3 a. Conceptual drawing of the prototype THGEM detector (Byun et al, 2009), b. constructed and assembled prototype THGEM-based microdosimetric detector

Fig. 2.1 a. Detector chamber lid design, b. Detector chamber baseplate design 18

Fig. 2.2 Multi-pin installation on the detector chamber baseplate

Fig. 2.3 a. Fabricated detector chamber lid and air side of the baseplate, b. Fabricated detector chamber baseplate (vacuum side)

Fig. 2.4 a. Fabricated detector chamber baseplate with 3 signal input (vacuum side) b. Assembled detector chamber with 3 signal input

Fig. 2.5 Detector Chamber baseplate design with 3 signal input

Fig. 2.6 A-150 layer as a cathode a. Designed, b \& c. Fabricated 22

Fig. 2.7 Rexolite layer as sensitive volume a. designed, b \& c. fabricated 23

Fig. 2.8 a. Old THGEM (fabricated by MyRoPCB Company, China), b. New THGEM (fabricated by Milplex Company, Canada), c. THGEM holes within a sensitive volume under a microscope, d. Typical acceptable clean and uniform THGEM holes, e. Typical imperfections of THGEM holes, f. Sparked induced damaged THGEM hole, g. A dusty layer on the surface of THGEM, h. Zoom in view of dust on the surface under the microscope

Fig. 2.9 Readout board layer a. designed, b. fabricated 27

Fig. 2.10 HV divider board 28

Fig. 2.11 Exploded view of the detector final design 29 
Fig. 2.12 High voltage connections circuit diagram for single THGEM layer

Fig. 2.13 Single volume detector assembly, a. 3 BNCs soldered to readout board for signal collection, b. Final detector assembly

Fig. 2.14 a. \& b. A 2-dimential multi-volume detector assembly, c. sealed and closed detector, d. \& e. Multi-pin plug (RG-170 cable soldered to the pin and inserted into the plug), f. custom made required signal cables: multi-pin plug - 9 BNCs, 9 signal cables

LIMO - BNC

Fig. 2.15 Double layer THGEM assembly a. Top view, b. Side view 33

Fig. 2.16 High voltage connections circuit diagram for double THGEM layers 34

Fig. 2.17 Array of $3 \times 3$ gas cavities with diameter of a. $2.5 \mathrm{~mm}$ and b. $1 \mathrm{~mm}$ 35

Fig. 2.18 Vacuum pump and filling gas station 37

Fig. 3.1 Block diagram of the pulse processing system and prototype MSI-DSP, showing the major functional sections 40

Fig. 3.2 a. MSI-DSP prototype printed circuit board, b. MSI-DSP prototype box 41

Fig. 3.3 Experimental setup using NaI detector, MSI-DSP, ORTEC DSPEC and Analogue signal processing systems

Fig. 3.4 MSI-DSP controller interface 43

Fig. 3.5 MSI-DSP display screen 44

Fig. 3.6 MSI-DSP gain setting block diagram

Fig. 3.7 a. Gamma-ray spectrum for low count rate: $3.1 \mathrm{kcps}$, b. Zoom in of lower channel numbers to show ${ }^{137} \mathrm{Cs}$ peak, c. Zoom in of higher channel numbers to show ${ }^{60} \mathrm{Co}$ peak ....47

Fig. 3.8 a. Gamma-ray spectrum for low count rate: $41 \mathrm{kcps}$, b. Zoom in of lower channel numbers to show ${ }^{137} \mathrm{Cs}$ peak, c. Zoom in of higher channel numbers to show ${ }^{60} \mathrm{Co}$ peak ....48

Fig. 3.9 a. Gamma-ray spectrum for low count rate: $76 \mathrm{kcps}$, b. Zoom in of lower channel numbers to show ${ }^{137} \mathrm{Cs}$ peak, c. Zoom in of higher channel numbers to show ${ }^{60} \mathrm{Co}$ peak ....49 
Fig. 3.10 a. Gamma-ray spectrum for low count rate: $142 \mathrm{kcps}$, b. Zoom in of lower channel numbers to show ${ }^{137} \mathrm{Cs}$ peak, c. Zoom in of higher channel numbers to show ${ }^{60} \mathrm{Co}$ peak 50

Fig. 3.11 Rate performances of MSI-DSP vs. DSPEC and Analogue systems 51

Fig. 3.12 Dead time of MSI-DSP vs. DSPEC and Analogue systems for different count rates

Fig. 3.13 Resolution of MSI-DSP vs. DSPEC and Analogue systems for different count rates

Fig. 3.14 a. A board of MMI-DSP consist of 5 channels, b. Wiring and assembly of the board on a 19-inch rack panel (top view), c. Front view, showing the BNC and power connections

Fig. 3.15 Design drawing of MMI-DSP mounting panel 55

Fig. 3.16 Experimental setup for testing MMI-DSP(5) 5-channel 56

Fig. 3.17 ${ }^{137} \mathrm{Cs}$ gamma spectrum using MMI-DSP(5) 56

Fig. 3.18 a. ${ }^{60} \mathrm{Co}$ gamma spectrum with a pulser peak at channel 900 using MSI-DSP, MMI-DSP(5) and DSPEC, b. Zoom in of spectra 57

Fig. 3.19 a. Assembly and wiring of MMI-DSP(10), b. Front panel view 58

Fig. 4.1 Tandetron Accelerator floor plan 62

Fig. 4.2 Layout of the Tandetron neutron irradiation facility at McMaster University ........ 63

Fig. 4.3 Schematic of experimental setup for single volume detector configuration 65

Fig. 4.4 a. Single volume detector placed in the irradiation cavity connected to the ORTEC charge sensitive preamplifier, b. Control room: accelerator operator desk and electronics c. electronics NIM bin: dual HV, pulser, amplifier 66

Fig. 4.5 Prototype custom made single input DSP (MSI-DSP) 67

Fig. 4.6 Schematic of experimental setup for multi-volume detector configuration 69 
Fig. 4.7 Experimental setup for multi-volume detector and multi-input MMI-DSP: 2D THGEM detector is placed in the irradiation cavity and connected to CAEN 8 channels and 1 channel charge sensitive preamplifier ....................................................................................................... 70

Fig. 5.1 ${ }^{244} \mathrm{Cm}$ internal Alpha source spectrum with Gaussian peak fit ….................................... 72

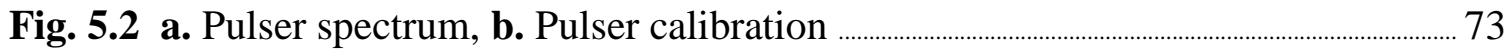

Fig. 5.3 Typical 1/2" TEPC a. raw spectrum, b. microdosimetric spectrum ……….................. 75

Fig. 5.4 A typical MATLAB output for pulser calibration of multi-volume detector ........ 77

Fig. 6.1 Experimental setup for dosimetry study using commercial TEPC. a. Far West 1/2" TEPC, b. The water bag placed around the detector in the arm access hole. Detector is connected to preamp and HV

Fig. 6.2 Microdosimetric spectra collected at the center of the irradiation position for different proton energies

Fig. 6.3 Microdosimetric spectra collected at various lateral positions for $\mathrm{E}_{\mathrm{p}}=2.3 \mathrm{MeV}$

Fig. 6.4 Microdosimetric spectra collected at various lateral positions for $\mathrm{E}_{\mathrm{p}}=1.95 \mathrm{MeV}$

Fig. 6.5 Microdosimetric spectra collected at various lateral positions for $\mathrm{E}_{\mathrm{p}}=2 \mathrm{MeV}$ 86

Fig. 6.6 Microdosimetric spectra collected at various lateral positions for $\mathrm{E}_{\mathrm{p}}=2.1 \mathrm{MeV}$

Fig. 6.7 Microdosimetric spectra collected at various lateral positions for $\mathrm{E}_{\mathrm{p}}=2.15 \mathrm{MeV}$

Fig. 6.8 Microdosimetric spectra collected at various lateral positions for $\mathrm{E}_{\mathrm{p}}=2.2 \mathrm{MeV}$

Fig. 6.9 Microdosimetric spectra collected at various lateral positions for $\mathrm{E}_{\mathrm{p}}=2.5 \mathrm{MeV}$

Fig. 6.10 Neutron fluence spectra per unit lethargy per unit proton charge simulated at two different positions: a. the center of cavity and b. $6 \mathrm{~cm}$ shifted towards arm access for various incident proton energies 
Fig. 6.11 Neutron fluence spectra per unit lethargy per unit proton charge simulated at various lateral positions along the arm for: $\mathbf{a} . \mathrm{E}_{\mathrm{p}}=2 \mathrm{MeV}$ and b. $\mathrm{E}_{\mathrm{p}}=2.3 \mathrm{MeV}$

Fig. 6.12 Neutron weighting factor as a function of the lateral position for all proton energies

Fig. 6.13 Spatial distribution of total equivalent dose for different proton energies (from 1.9 to $2.5 \mathrm{MeV}$ )

Fig. 7.1 a. An indication of discharge on oscilloscope, b. burnt Rexolit, c. THGEM, d. burnt readout board, e. burnt THGEM hole, f. rim-hole eccentricity . 104

Fig. 7.2 Growth of preamplifier (CAEN) (channel 1) and amplifier (gain of 50) (channel 2) signal on the oscilloscope with increasing the THGEM applied voltage a. Noise level without beam, b. $\mathrm{HV}_{\mathrm{THGEM}}=760 \mathrm{~V}$, c. $\mathrm{HV}_{\mathrm{THGEM}}=860 \mathrm{~V}$, d. $\mathrm{HV}_{\mathrm{THGEM}}=920 \mathrm{~V}$, e. $\mathrm{HV}_{\mathrm{THGEM}}=980 \mathrm{~V}$, f. $\mathrm{HV}_{\mathrm{THGEM}}=1020 \mathrm{~V}$ 106

Fig. 7.3 Detector signal amplitude vs. applied high voltage across the THGEM 107

Fig. 7.4 Detector gain stability over half an hour time period (27 Nov. 2014 data) a. linear scale, b.log scale 108

Fig. 7.5 Detector gain stability over half an hour time period after 2 hours from the beginning of measurement (27 Nov. 2014 data) a. linear scale, b. log scale 109

Fig. 7.6 Detector gain stability over 2 hours from the beginning of measurement (27 Nov. 2014 data)

Fig. 7.7 Detector gain stability over 3 weeks

Fig. 7.8 Raw spectrum of new THGEM\#1 which was sparked

Fig. 7.9 Liquid/oil spots on THGEM \#1 from a new batch caused spark during measurement

Fig. 7.10 Detector gain stability over 20 minutes time period a. linear scale, b.log scale

Fig. 7.11 Detector gain stability over 2 weeks a. Full scale, b. Zoom in

Fig. 7.12 THGEM detector microdosimetric spectrum using different THGEMs from old batch compared to commercial 1/2" TEPC and 2" TEPC 
Fig. 7.13 THGEM detector microdosimetric spectrum using different THGEMs from new batch compared to commercial 1/2" TEPC and 2" TEPC

Fig. 7.14 THGEM detector microdosimetric spectrum using THGEMs from old batch vs. THGEM from new batch compared to commercial 1/2" TEPC and 2" TEPC

Fig. 7.15 a. A THGEM in $20 \%$ Nitric Acid, b. the THGEM in the Nitric Acid in the ultrasonic bath for $20 \mathrm{~min}$, c. cleaned THGEMs in the oven d. THGEM surface and holes under microscope

Fig. 7.16 THGEM detector microdosimetric spectrum for the chemically cleaned THGEM \#6 compared to the spectrum for THGEM \#5 and \#10 from the new batch and commercial $1 / 2 "$ TEPC. $\Delta \mathrm{V}_{\text {THGEM } \# 6}=883.33 \mathrm{~V}, \Delta \mathrm{V}_{\text {THGEM }}=850 \mathrm{~V}, \Delta \mathrm{V}_{\text {THGEM }}=850 \mathrm{~V}$

Fig. 7.17 THGEM detector raw spectrum using a. DSPEC with three different gain settings, b. MSI-DSP with fixed gain of 10 123

Fig. 7.18 THGEM detector microdosimetry spectra: comparison between DSPEC and MSI-DSP

Fig. 7.19 2" TEPC microdosimetry spectra: comparison for DSPEC between $2 \mathrm{k}$ and $16 \mathrm{k}$ channel

Fig. 7.20 2" TEPC microdosimetry spectra: comparison for MSI-DSP between 60 and 30 bin number of bins 126

Fig. 7.21 Array of $3 \times 3$ detectors: 9 individual detectors numbered accordingly for convenience

Fig. 7.22 Detector signal in proportional region .129

Fig. 7.23 THGEM detector (D5) raw spectrum using DSPEC and CAEN preamplifier a. full scale, b. half-scale

Fig. 7.24 THGEM detector (D5) spectrum acquired using DSPEC and ORTEC preamplifier at different times for $10 \mathrm{~min}$ each a. Raw data, b. microdosimetric spectra...132

Fig. 7.25 2D THGEM detector with THGEM \#9 data compared to reference data using DSPEC: Raw spectra

Fig. 7.26 2D THGEM detector with THGEM \#9 data compared to reference data using DSPEC: Microdosimetric spectra 
Fig. 7.27 The order of detectors connections to the MMI-DSP. The array of $3 \times 3$ detector: 9 individual detectors numbered and connected to the channels of MMI-DSP 136

Fig. 7.28 The 2D THGEM detector raw spectrum using the MMI-DSP $\left(E_{p}=2.3 \mathrm{MeV}\right.$ and $\mathrm{I}_{\mathrm{p}}=50 \mu \mathrm{A}$ ). The plot on the left side in each row shows the raw spectrum of each detector and the plot on the right side is the corresponding pulser calibration for that detector and MMI-DSP input a. D1, b. D2, c. D3, d. D4, e. D5, f. D6, g. D7, h. D8, I. D9

Fig. 7.29 The 2D THGEM detector raw spectrum with pulser peak using the MMI-DSP $\left(E_{p}=2.3 \mathrm{MeV}\right.$ and $I_{p}=50 \mu$ A). a. D1, b. D2, c. D3, d. D4, e. D5, f. D6, g. D7, h. D8, I. D9

Fig. 7.30 THGEM detector (D5) a. raw spectrum and b. microdosimetric spectrum, acquired using $\mathrm{Ch} 1$ and $\mathrm{Ch} 6$ on the MMI-DSP and DSPEC individually 144

Fig. 7.31 THGEM detector (D1) a. raw spectrum and b. microdosimetric spectrum, acquired using $\mathrm{Ch} 1$ and $\mathrm{Ch} 5$ on the MMI-DSP individually 145

Fig. 7.32 MMI-DSP channels connecting individually to different detectors to check each channel on each board, a. THGEM detector (D1) raw spectrum acquired using Ch1 and Ch5 on the MMI-DSP, b. D2 raw spectrum acquired using Ch2 and Ch8, c. D3 raw spectrum acquired using Ch4 and Ch9, d. D4 raw spectrum acquired using Ch4 and Ch7, e. D6 raw spectrum acquired using $\mathrm{Ch6}$ and $\mathrm{Ch5}$, f. D7 raw spectrum acquired using $\mathrm{Ch} 7$ and $\mathbf{C h 4}$, g. D8 raw spectrum acquired using $\mathrm{Ch} 8$ and $\mathrm{Ch} 2$, h. D9 raw spectrum acquired using $\mathrm{Ch} 9$ and $\mathrm{Ch} 5$ 146

Fig. 7.33 Pulser calibration for each MMI-DSP channels connecting to different detectors a. $\mathrm{Ch} 1$, b. $\mathrm{Ch} 2$, c. $\mathrm{Ch} 4$, d. $\mathrm{Ch} 5$, e. $\mathrm{Ch} 6$, f. $\mathrm{Ch} 7$, g. $\mathrm{Ch} 8$, h. $\mathrm{Ch} 9$

Fig. 7.34 2D THGEM detector position inside the irradiation cavity at a \& c. regular position and b \& d. $90^{\circ}$ rotated position 148

Fig. 7.35 2D THGEM detector with THGEM \#1 raw spectrum compared to the $90^{\circ}$ rotation

Fig. 7.36 2D THGEM detector with THGEM \#1 microdosimetric spectrum compared to the $90^{\circ}$ rotation 150

Fig. 7.37 Comparison of the THGEM detector (D5) microdosimetric response acquired with different THGEMs (\#9, \#10 and \#11) for $\mathrm{E}_{\mathrm{p}}=2.3 \mathrm{MeV}$ and $\mathrm{I}_{\mathrm{p}}=50 \mu \mathrm{A}$ 151 
Fig. 7.38 Comparison of the 2D THGEM detectors microdosimetric response acquired with different THGEMs (\#9, \#10, \#11) for $E_{p}=2.3 \mathrm{MeV}$ and $\mathrm{I}_{\mathrm{p}}=50 \mu \mathrm{A}$

Fig. 7.39 2D THGEM detector with THGEM \#10 raw spectrum for $E_{p}=2.3 \mathrm{MeV}$ and two different proton current of $\mathrm{I}_{\mathrm{p}}=50 \mu \mathrm{A}$ and $\mathrm{I}_{\mathrm{p}}=150$

Fig. 7.40 2D THGEM detector with THGEM \#10 raw spectrum, lower lineal energy part of spectrum for $E_{p}=2.3 \mathrm{MeV}$ and two different proton currents of $I_{p}=50 \mu \mathrm{A}$ and $\mathrm{I}_{\mathrm{p}}=150 \mu \mathrm{A}$

Fig. 7.41 Count rate comparison between $50 \mu \mathrm{A}$ and $150 \mu \mathrm{A}$ proton current $\left(\mathrm{E}_{\mathrm{p}}=2.3 \mathrm{MeV}\right)$ for all detectors (Except D3)

Fig. 7.42 $2 \mathrm{D}$ THGEM detector with THGEM \#10 raw spectrum for $\mathrm{I}_{\mathrm{p}}=50 \mu \mathrm{A}$ and three different proton energies of $\mathrm{E}_{\mathrm{p}}=2 \mathrm{MeV}, \mathrm{E}_{\mathrm{p}}=2.3 \mathrm{MeV}$ and $\mathrm{E}_{\mathrm{p}}=2.5 \mathrm{MeV}$ 158

Fig. 7.43 2D THGEM detector with THGEM \#10 raw spectrum, lower lineal energy part of spectrum for $\mathrm{I}_{\mathrm{p}}=50 \mu \mathrm{A}$ and three different proton energies of $\mathrm{E}_{\mathrm{p}}=2 \mathrm{MeV}, \mathrm{E}_{\mathrm{p}}=2.3 \mathrm{MeV}$ and $\mathrm{E}_{\mathrm{p}}=2.5 \mathrm{MeV}$

Fig. 7.44 Count rate comparison between $2 \mathrm{MeV}, 2.3 \mathrm{MeV}$ and 2.5 $\mathrm{MeV}$ proton energies $\left(\mathrm{I}_{\mathrm{p}}=50 \mu \mathrm{A}\right)$ for all detectors (Except D3)

Fig. 7.45 Two different induction gap (between readout board and THGEM layer) a. $1 \mathrm{~mm}$ spacer gap, b. $2 \mathrm{~mm}$ square gap

Fig. 7.46 Microdosimetric spectrum of THGEM \#11 acquired with ORTEC DSPEC (16k) for two different induction gap size

Fig. 7.47 The THGEM detector (D5) signal response for a. single-layer THGEM \#11 with $\Delta \mathrm{V}_{\text {THGEM }}=850 \mathrm{~V}\left(\mathrm{HV}_{\text {Cathode }}=1120 \mathrm{~V}\right.$ and $\left.\mathrm{HV}_{\mathrm{THGEM}}=1020 \mathrm{~V}\right)$ : preamplifier $(\mathrm{CAEN})$ (channel 1) and amplifier (gain = 20) (channel 2), b. double-layer THGEM \#11 \& \#12 with $\Delta \mathrm{V}_{\text {THGEM1\&2 }}=733 \mathrm{~V}\left(\mathrm{HV}_{\text {Cathode }}=1760 \mathrm{~V}\right.$ and $\left.\mathrm{HV}_{\mathrm{THGEM}}=1660 \mathrm{~V}\right)$ : preamplifier $(\mathrm{CAEN})$ and amplifier $($ gain $=2)$

Fig. 7.48 Microdosimetric spectra acquired with ORTEC DSPEC (16k) for single-layer THGEM \#11 vs double-layer THGEM \#11 and \#12 164

Fig. 7.49 Background Monitoring. Raw spectra acquired with double-layer THGEM \#11 and \#12

Fig. 7.50 2D double THGEM detector with THGEM \#11 \& \#12 raw spectrum for $\mathrm{I}_{\mathrm{p}}=50$ $\mu \mathrm{A}$ and $\mathrm{E}_{\mathrm{p}}=2.3 \mathrm{MeV}$ compared to the central detector (D5) 167 
Fig. 7.51 2D double THGEM detector with THGEM \#11 \& \#12 microdosimetric spectrum for $\mathrm{I}_{\mathrm{p}}=50 \mu \mathrm{A}$ and $\mathrm{E}_{\mathrm{p}}=2.3 \mathrm{MeV}$ compared to the central detector (D5) 168

Fig. 7.52 The absorbed dose distribution inside the $5 \times 5 \mathrm{~cm}^{2}$ area of the 2D THGEM detector (an array of $3 \times 3$ gas cavity)

Fig. 7.53 THGEM detector assembly with $2.5 \mathrm{~mm}$ diameter gas cavities a. number of THGEM holes within the cavities, b. \& c. detector assembly, d. detector signal observed on the oscilloscope

Fig. 7.54 THGEM detector with $2.5 \mathrm{~mm}$ diameter gas cavities response for a. raw spectra for different applied high voltages across the THGEM, b. raw spectra for different proton energies, c. microdosimetric spectra for different applied high voltages across the THGEM, d. microdosimetric spectra for different proton energies 174

Fig. 7.55 THGEM detector assembly with $1 \mathrm{~mm}$ diameter gas cavities, a. double-THGEM assembly using $2 \mathrm{~mm}$ square gap between THGEMs b. number of THGEM holes within the cavities, c. detector assembly, d. preamp and amplifier (gain of 25) signals 175

Fig. 7.56 THGEM detector with $1 \mathrm{~mm}$ diameter gas cavity response a. raw spectrum, b. microdosimetric spectrum for $\mathrm{E}_{\mathrm{p}}=2.1 \mathrm{MeV}$ and $\mathrm{I}_{\mathrm{p}}=300 \mu \mathrm{A}$ 176 


\section{List of Tables}

Table 1-1 Elemental composition of TE compounds in \% by weight

Table 3.1 The real-time performance of the pulse pile-up recovery algorithm in MSI-DSP, in terms of rate performances, dead time and resolution for the $662 \mathrm{keV}$ energy peak from ${ }^{137} \mathrm{Cs}$

Table 5-1. Mass and dose conversion coefficients for each detector 75

Table 6-1. Neutron absorbed dose values

Table 6-2. Gamma absorbed dose values

Table 6-3. Neutron equivalent dose values 96

Table 6-4. Gamma equivalent dose values 96

Table 6-5 Total equivalent dose values 97

Table 6-6. Neutron equivalent dose values for outside 98

Table 6-7. Gamma equivalent dose values for outside 98

Table 7-1. Applied high voltages and corresponding detector signal amplitude data ...... 105

Table 7-2. Comparison of number of channels that fall into a logarithmic bin for $16 \mathrm{k}$ and 2k ADC channels

Table 7-3. Detectors (THGEM \#9) count rate for $E_{p}=2.3 \mathrm{MeV}$ and $I_{p}=50 \mu A$

Table 7-4. Central detector (D5) count rate for various proton energies and $\mathrm{I}_{\mathrm{p}}=50 \mu \mathrm{A}$

Table 7-5. Detectors (THGEM \#9) count rate for $E_{p}=2.3 \mathrm{MeV}$ and $\mathrm{I}_{\mathrm{p}}=50 \mu \mathrm{A}$

Table 7-6 Channel numbers corresponding to the redistributed y value for each detector based on the related MMI-DSP input pulser calibration

Table 7-7. Measurements carried out for single layer THGEM \#11 and double layer THGEM\#11 \&\#12 for different induction gap size and type

Table 7-8. Count rate for background monitoring 165 
Table 7-9. Dose rate for D5 obtained with different single and double layer THGEMs for $\mathrm{E}_{\mathrm{p}}=2.3 \mathrm{MeV}$ 169

Table 7-10. Dose rate distribution for D5 obtained with different single and double layer THGEMs for $\mathrm{E}_{\mathrm{p}}=2.3 \mathrm{MeV}$ 



\section{Chapter 1}

\section{Introduction}

1.1 Microdosimetry Applications

1.2 Microdosimetric Quantities

1.3 Primary Interactions of Radiation with Matter

1.4 Tissue Equivalent Proportional Counters (TEPC)

1.4.1 Traditional Generation of TEPC

1.4.2 New Generation of TEPC

1.5 Thick Gas Electron Multipliers (THGEM)

1.6 Motivation, Goal and Approach of the Thesis 


\section{Introduction}

Interactions of ionizing radiations with biological materials can induce a wide variety of effects due to damage to individual cells either singly or in multiples. Cell killing, mutation, chromosome aberration and carcinogenesis are some of the main examples of these biological effects. Humans can be irradiated either by environmental, occupational, diagnostic or therapeutic exposures. Hence, the precise understanding of the mechanism by which the ionizing radiations induce these detrimental effects is crucial. Determination of these biological effects is not possible by merely the absorbed dose information. Additional concepts and quantities are required to specify the distribution of energy deposition on microscopic scales. All physical descriptions of these microscopic patterns establish the field of microdosimetry in general, whose techniques and methods provide a physical description of radiation fields and their interactions with matter (Goodhead, 1987).

Experimental microdosimetry is the study of the effects of ionizing radiations on biological targets by investigating the probabilistic distribution of energy deposition events at cellular and subcellular levels. Microdosimetry in its present sense was originated more than 60 years ago by Harold H. Rossi when he studied energy deposition in small irradiated masses (Rossi and Zaider 1996). He realized that the range of energy depositions observed in the micrometric scale is characteristic of the energy deposited in critical biological cells resulting in the initiation of biological damage (Kellerer, 1985). Rossi and his colleagues constructed a novel spherical tissue-equivalent proportional counter (TEPC) to evaluate linear energy transfer (LET) in an unknown radiation field.

The invention of TEPCs simulating $\mu \mathrm{m}$ diameter volumes, resulted in a practical demonstration of the stochastic nature of energy deposition in small regions. TEPCs not only contributed to a better understanding of the interactions between ionising radiation and biological targets, but also have had a significant impact on applied radiation dosimetry. TEPCs have been proven to be the ideal detectors for monitoring complex radiation fields. However depending on the application they turned out to have limitations.

To overcome some of the limitations of the standard TEPCs which will be addressed later in this chapter, an advanced two-dimensional TEPC based on Thick Gas Electron 
Multipliers (THGEM) was designed, constructed and implemented at McMaster University by the author of this thesis and the detailed explanation of the research project and its achievements will be the purpose of this dissertation.

\subsection{Microdosimetry Applications}

There are many biological applications in which conventional dosimetry techniques provide a sufficient physical description of the radiation field and biological material interaction. However, in many cases specifically in the presence of mixed radiation fields, such as beams of photons, neutrons and heavy particles, absorbed dose information alone is not adequate. To understand the mechanism of radiations action with different quality, especially at the cellular and subcellular levels, microdosimetric description of the radiation has to be employed (Booz, 1984). As microdosimetry is able to provide a quantitative dose measurement along with a dose spectrum, it has been applied to a wide variety of fields, mainly radiobiology, radiation protection, aviation dosimetry, radiotherapy, etc.

Irrespective of the radiation type, ionizing radiations cause ionization or excitation with the same number of these products per unit energy deposited in a particular irradiated material. However what makes the different radiation effectiveness is the unequal spatial distributions of energy deposition in charged particle tracks. Explicitly, in radiation biology the damage to DNA and the spatial distribution of the affected molecules are the causes of radiobiological effects. Microdosimetry, which is a purely physics activity, includes no biology. To investigate the precise link between radiation physics and cellular radiobiology the "theory of dual radiation action" (TDRA) was proposed by Kellerer and Rossi in 1972 (Kellerer and Rossi, 1972 and 1978) to enable the explanation of the mechanism of radiation action for a wide variety of biological effects. A full description and formulation of TDRA can be founded in different literature (Goodhead, 1982; Kellerer and Rossi, 1972 and 1978).

After the publication of the first paper about microdosimetry, the relevance of this approach was immediately apparent in radiation protection where low doses, small number 
of events and different type of radiation are the primary concerns (Wambersie et al, 1990; Booz, 1984). Therefore it is necessary to formulate the quantity that weight absorbed doses by their biological effectiveness. ICRU defined the dose equivalent, $H$, as (ICRU, 1980 and 1993):

$$
H=Q \times D
$$

where $Q$ is the quality factor, which is not in itself an RBE (radiation biological effectiveness) but rather a synthesis of RBE values for that radiation. $D$ is the absorbed dose in tissue at the point of interest. Since $Q$ is a dimensionless quantity, $H$ has the same unit as $D$. To minimize confusions in the case of $Q=1$, a different name was admitted for dose equivalent as Sievert (Sv). Quality factors are given as a function of linear energy transfer (LET) according to ICRP 60 (ICRP, 1991), and as a function of lineal energy according to ICRU 40 (ICRU, 1986). As lineal energy is the microdosimetric quantity measured with TEPC, hence the dose equivalent can be determined directly by weighting the absorbed dose distribution, which can be measured as a function of lineal energy, with the appropriate quality factor related to each value of lineal energy (Waker et al, 2002).

Experimental microdosimetry has played a critical role in the evaluation of air-crew exposure and in the verification of cosmic radiation transport calculations in aviation dosimetry (Braby, 2015; Waker et al, 2002). Also for neutron monitoring in nuclear power plants which has been a significant challenge in operational health physics, experimental microdosimetry can be employed, however a detector design should have an improvement for higher sensitivity and neutron energy response (Waker et al, 2002).

In radiotherapy, where the doses are relatively high, the possible application of microdosimetry became more evident with the advent of neutron therapy (and high LET therapy). For low LET radiation the differences in RBE are small in contrast to high LET radiation for which the differences in RBE are considerable. Fast Neutron Therapy (FTN), Boron Neutron Capture Therapy (BNCT) (Wuu, 1992; Burmeister, 1999, 2001 and 2002; Santa Cruz and Zamenhof, 2004; Santa Cruz, 2016) and proton and heavy ion therapy (Rollet et al, 2011; Burigo et al, 2014) are the high LET radiation therapies in which microdosimetric methods have been applied. The most challenging aspect of these 
techniques is to determine the absorbed dose delivered to the patient accurately and to predict the associated RBE (Burmeister et al, 2001). To this end, microdosimetric measurements can provide the required information and a reliable description of the therapeutic beam quality for the optimization of the treatment planning (Rollet et al, 2011; Waker and Marco, 1992; Kiliauga et al, 1996). Therefore, microdosimetry is a priceless tool to understand and explain results that apparently have no suitable explanation by considering only average quantities, like absorbed dose or LET. It also delivers a proper measuring methodology useful for performing quality control tests of therapeutic radiation beams, where differences in the acquired microdosimetric spectra are almost always associated with variations in radiobiological properties of the beams (Santa Cruz, 2016).

\subsection{Microdosimetric Quantities}

Ionizing radiations consisting of charged particles and uncharged particles are capable of causing ionization by primary or secondary processes. An event in which energy is imparted to the matter in a defined volume by an ionizing particle or its secondaries, is denoted as an energy deposition event. The energy imparted, $\varepsilon$, to a matter, which is the sum of all energy transfers within a specified site $\left(\varepsilon_{i}\right)$, is subject to random fluctuations which become significant if the mass of interest is small.

$$
\varepsilon=\sum \varepsilon_{i} \quad\left(\varepsilon_{i}: \text { in a specified site }\right) \quad[\mathrm{J} \text { or } \mathrm{eV}]
$$

The specific energy, $z$, is another stochastic quantity, which is $\varepsilon$ divided by the mass of matter $m$ :

$$
z=\frac{\varepsilon}{m} \quad\left[\mathrm{~J} \mathrm{~kg}^{-1} \text { or } \mathrm{Gy}\right]
$$

The lineal energy, $y$, was originally introduced by Rossi and his colleagues as a random analogue to LET. It is a stochastic quantity and is defined as the energy imparted to the matter in a volume by a single energy-deposition event divided by the mean chord length in that volume:

$$
y=\frac{\varepsilon}{\bar{l}} \quad\left[\mathrm{~J} \mathrm{~m}^{-1} \text { or } \mathrm{keV}_{\mu \mathrm{m}^{-1}}\right]
$$


The mean chord length in a volume is the mean length of randomly oriented chords in that volume. For a convex body, it is defined as (Rossi and Zaider, 1996):

$$
\bar{l}=\frac{4 V}{S}
$$

where $V$ and $S$ are the volume and surface area of the body, respectively. For the spherical and right cylinder volumes with diameter of $d$, which we will use further, the mean chord length can be calculated as:

$$
\bar{l}=\frac{2 d}{3}
$$

When particles interact with a given volume they can release different quantities of energies with different probabilities. Dose-mean lineal energy is defined as:

$$
\bar{y}_{D}=\int_{0}^{\infty} y d(y) d y
$$

In general convention microdosimetric spectra are plotted in terms of $y d(y)$ against lineal energy in semi-log plots. As the $y d(y)$ curve in logarithmic lineal energy scale is invariant with respect to the absorbed dose, it is generally used for presenting microdosimetric data. Nevertheless, $y N(y)\left(N(y)\right.$ denotes the number of counts in the $i^{\text {th }}$ logarithmic bin) can be used rather than $y d(y)$ as the integral of the $y N(y)$ is proportional to the absorbed dose, which is a better way to show the spatial distribution of the absorbed dose spectrum. Then the absorbed dose which is defined as the total deposited energy divided by mass is given as below ( $y$ is in $\mathrm{keV}_{\mu \mathrm{m}}^{-1}$ ):

$$
D=1.602 \times 10^{-16} \frac{\bar{l}}{\rho V} \sum_{i} y_{i} N\left(y_{i}\right) \quad[\mathrm{Gy}]
$$

where $\rho$ and $V$ are the density of the gas and volume of the gas cavity. The dose calculation for the purpose of this thesis will be explained in much more detail in the data analysis chapter (chapter 5). 


\subsection{Primary Interaction of Radiation with Matter}

A knowledge of the fundamental interactions of radiation with matter is essential for microdosimetry. The sources of charged particles could be 1) photons (which for instance, generate energetic electrons), 2) neutrons which produce protons, other nuclear recoils and other charged particles via nuclear reactions and 3) charged particles themselves.

\section{Photons}

Within the photon energy range of up to about $8 \mathrm{MeV}$, all of the photon's energy-loss interactions take place by three competing interactions; photoelectric effect, Compton scattering and pair production. The cross section for photo electric effect and pair production depends upon the atomic number of the absorber. For the relatively light elements, which are of greatest interest in microdosimetry $\left({ }^{1} \mathrm{H},{ }^{12} \mathrm{C},{ }^{14} \mathrm{~N},{ }^{16} \mathrm{O}\right)$, the photo electric effect is dominant for photons up to $40 \mathrm{keV}$, and Compton scattering predominates from $40 \mathrm{keV}$ to $10 \mathrm{MeV}$. (ICRU, 1969; ICRU, 1970b; ICRU, 1973)

Compton scattering, in which a part of the primary photon's energy is transferred to a single atomic electron and a Compton scattered photon is emitted, is the primary interaction of gamma rays within the sensitive volume of the detector, resulting in release of secondary charged particles whose energy are deposited within the sensitive volume (TissueEquivalent (TE) gas) of the detector (Waker, 1995).

\section{Neutrons}

As neutrons pass through hydrogenous material, including water and the human body, secondary charged particles, such as recoil protons, heavy recoil nuclei and products of nuclear reactions, are mainly produced by elastic scattering, inelastic scattering, capture processes (important for thermal neutrons) and spallation (significant at neutron energies above $10 \mathrm{MeV}$ ). In the energy range of neutrons generated by the radiation source in this study (which will be explained in chapter 4 later on), the dominant interactions are elastic and inelastic scattering of neutrons with the TE gas molecules. The dominant interactions of 100 to few 100s keV neutrons occur with hydrogen component of the TE propane based 
gas $\left(10.3 \%{ }^{1} \mathrm{H}, 56.9 \%{ }^{12} \mathrm{C}, 29.3 \%{ }^{16} \mathrm{O}, 3.5 \%{ }^{14} \mathrm{~N}\right.$ or $\left.55 \% \mathrm{C}_{3} \mathrm{H}_{8}, 39.5 \% \mathrm{CO}_{2}, 5.5 \% \mathrm{~N}_{2}\right)$ (ICRU, 1983). However, heavy ion recoils become significant at higher neutron energies.

\subsection{Tissue Equivalent Proportional Counters (TEPC)}

TEPC is a type of proportional counter that has been widely used for microdosimetry (ICRU 36, 1983; Rossi and Zaider, 1996). This detector was originally developed by Rossi and Rozenzweig at Colombia University in the early 50's (Rossi and Rosenzweig, 1955; ICRU, 1983). Nowadays most counters in use are variation and modification of the Rossi counter. The design of a proportional counter for microdosimetric purposes is subject to some fundamental requirements, such as determination of the type of radiations that the counter will be irradiated with, TE material of construction, size of the simulating a microscopic tissue volume and finally the ease of construction. Proportional counters can be fabricated from a wide variety of metals and plastics, however for the microdosimetric objective, the tissue-equivalent plastic and gas compositions have to be employed. Table 1-1 shows the composition of TE plastics and gases which have been commonly used in TEPCs.

Table 1-1 Elemental composition of TE compounds in \% by weight

\begin{tabular}{|c|c|c|c|c|c|c|}
\hline Name & Reference & $\mathrm{H}$ & $\mathrm{C}$ & $\mathrm{N}$ & $\mathrm{O}$ & $\mathrm{F}$ \\
\hline TE conductor plastic $(\mathrm{A}-150)$ & Smathers et al, 1977 & 10.1 & 77.6 & 3.5 & 5.2 & 1.7 \\
\hline TE gas, Propane based & Srdoc, 1970 & 10.3 & 56.9 & 3.5 & 29.3 & - \\
\hline TE gas, Methane based & Rossi and Faila, 1956 & 10.2 & 45.6 & 3.5 & 40.7 & - \\
\hline
\end{tabular}

Due to the complete symmetry with respect to isotropic radiation, a sphere is the most typical shape commonly used to fabricate the sensitive volume of a TEPC. However the cylindrical counter is simplest to design and easiest to construct. For a right cylinder (a cylinder whose length is equal to its diameter) the mean chord length is the same as a sphere having the same diameter when subjected to isotropic radiation (Kellerer, 1981).

Typically when a Rossi proportional counter is exposed to a radiation field, radiation interacts with the gas molecules within the sensitive volume (TE gas) and surrounding wall, resulting in the generation of electron ion pairs. A proper electric field is required to 
accelerate the generated electron ion pairs toward their respective electrodes and induce gas multiplication to enable detection of the small number of ion pairs generated by incoming radiation. In the process of moving electrons toward the anode, additional free electrons are accelerated and generated by collisions with other neutral gas molecules, resulting in the Townsend Avalanche. Once all free electrons are collected at the anode the avalanche is terminated. In general, a charge that is proportional to the number of ion pairs created by traversing particles, is the physical quantity that is actually measured by a TEPC. Typically each interaction of the radiation with the counter gas gives rise to a measurable pulse of height approximately proportional to the energy deposited within the counter. (Knoll, 2010)

It is mostly the gas multiplication methodology that is different between the traditional and the new generation of TEPC. In the former the wire electrodes are used, and in the latter, Gas Electron Multipliers (GEM or THGEM) are used for the multiplication purpose. In the following, these two generations and techniques will be discussed.

\subsubsection{Traditional Generation of TEPC}

The traditional TEPCs consist of spherical shells, moulded of A-150 plastic as the counter wall. The anode of the detector is a fine wire, strung from pole to pole of the sphere. There are two methods to produce the constant electric field along the length of anode wire: 1) to center the anode in a cylindrical grid consisting of wire helix (Rossi and Rosenzweig, 1955) or 2) to use the field shaping electrodes (Benjamin, 1968). These detectors are commercially available and manufactured by Far West Technology Inc. in different sizes, such as 1/2" (Model LET-1/2 and LET-SW1/2), 2" (Model LET-2 and LET-SW2) and 5" (Model LET-SW5). In this research, Far West 1/2" and 2" TEPC were used to obtain the standard microdosimetric spectrum which was then used to validate the data obtained with the new prototype detector developed as the subject of this thesis. The experimental methodology and all the related settings will be fully explained in chapter 4 and 6 .

Operation, detector response and application of TEPCs have been well investigated in different research studies (Waker, 1985; Gerdung et al, 1995; Kliauga et al, 1995; Waker, 
1995; Badhwar et al, 2002; Aslam et al, 2003; Van Eijk, 2004; Zhang et al, 2014; Kowalski, 2015).

\subsubsection{New Generation of TEPC}

Although conventional TEPCs are the standard detector for microdosimetry, there are several limitations associated with their design and application. Mainly, due to their size, resulting extremely high detection count rate and consequently severe pileup and dead time issues, they cannot be operated in intense radiation fields, encountered in modern clinical radiation therapy facilities (Waker and Marco 1992). To overcome this limitation, there is a need to miniaturize the detector (Kliauga, 1996). However due to their electrode wire structure, it is extremely difficult and expensive to construct a small fine central wire in a small cavity. This causes many difficulties in construction of multi-element or 2dimensional counters. Hence, new types of TEPCs have been developed based on GEM or THGEM technologies which will be explained in the next section.

\subsection{THick Gas Electron Multipliers (THGEM)}

Gas Electron Multiplier (hereafter GEM) technology was invented in 1997 by Fabio Sauli. He introduced a new concept for gas multiplication in gas detectors: a composite grid consisting of two metal layers separated by a thin insulator, etched with a regular matrix of open channels and kept at a suitable difference of potential. When inserted in a gas detector on the path of drifting electrons, this causes the amplification of the charge moving through the channels. (Sauli, 1997) The GEM manufacturing technology has been developed at CERN by A. Gandi and R. D. Oliveira and the method of manufacturing a GEM was patented by CERN in 2013 (Oliveira et al, 2013). Fig. 1.1 a shows a picture of a typical GEM with thickness of $50 \mu \mathrm{m}$ and $70 \mu \mathrm{m}$ diameter holes with $140 \mu \mathrm{m}$ holes pitch. Once adequate voltage is applied across the conducting layers, the electric field generated across the holes as is illustrated in Fig. 1.1 b. Then electrons produced in the upper stream of the GEM are drifted into these holes and are accelerated through, resulting in an electron 
multiplication with effective gain well above $10^{2}$ (depending on the proper voltage across the GEM).

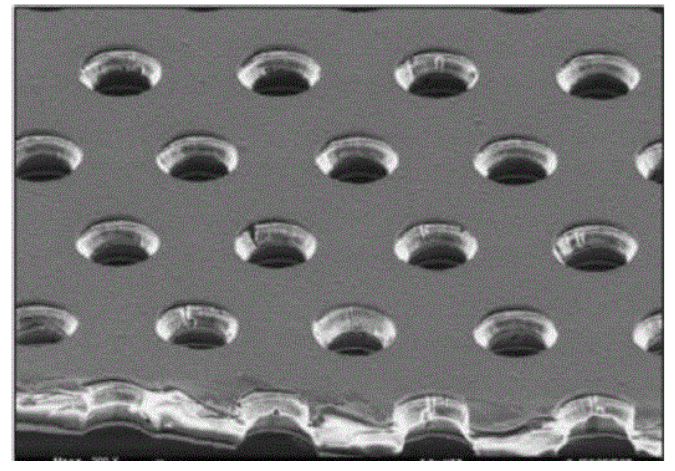

a.

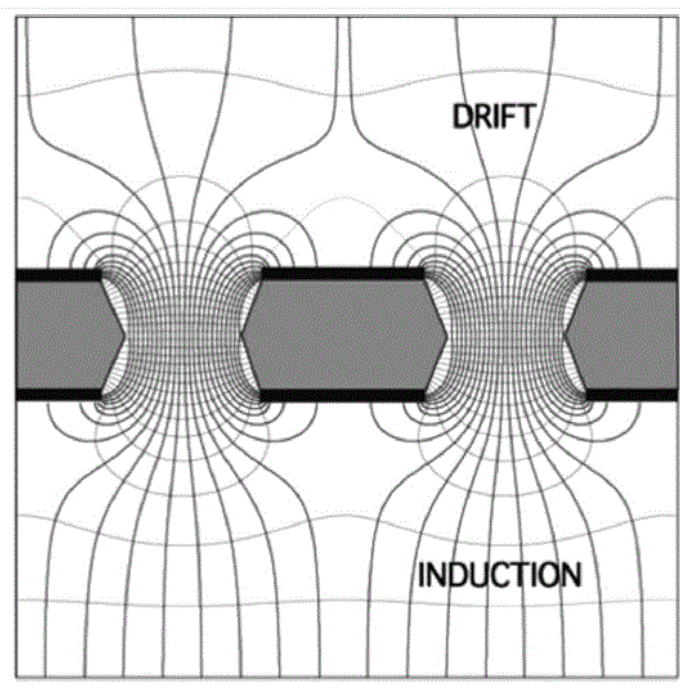

b.

Fig. 1.1 a. Electron microscope picture of a section of typical GEM electrode, $50 \mu \mathrm{m}$ thick. The holes pitch and diameter are 140 and $70 \mathrm{~mm}$, respectively, b. Electric field in the region of the holes of a GEM electrode (Sauli, 2016)

With the advent of GEMs, they have been widely used for different applications specifically gaseous detectors (Sauli, 2003; Ostling, 2006; Peskov et al, 2007; Maia et al, 2007; Marchiori, 2014). Specifically for microdosimetry purpose, Farahmand developed a mini multi-element TEPC based on GEM (Farahmand, 2004). Likewise in 2007 Seydaliev designed and constructed a GEM-based TEPC for neutron protection dosimetry (Seydaliev, 2007). Both results showed that the TEPC based on GEM is a good alternative for conventional TEPCs for microdosimetric measurements (Farahmand et al, 2003; Farahmand et al, 2004; Wang et al, 2008; Farahmand and De Nardo, 2015). More recently a Japanese group, developed a neutron detector based on GEM (Ohshita et al, 2010). Within the same group, Uno et al developed a two-dimensional imaging neutron detector based on GEM for the purpose of neutron radiography (Uno et al, 2012).

Another GEM-derivative that more recently has emerged is the thick GEM (hereafter THGEM), whose insulator is replaced by a thicker (in the order of sub $\mathrm{mm}$ to $\mathrm{mm}$ compared 
to $\mu \mathrm{m}$ in GEM) glass fibre-reinforced-epoxy plate and the holes, whose diameters are much bigger by a similar factor, are mechanically drilled (Periale et al, 2002 and 2003). Therefore THGEMs can be readily fabricated from any printed circuit boards (PCB) manufacturer, making it a very cost effective amplification technology. In addition, as the automatic drilling of the holes is a standard industry procedure, fabricating THGEMs with various holes' diameters and pitches, different shape, thickness and size is possible. As with GEM, careful cleaning, not introducing any sort of conducting debris or stains, should be used for THGEM as well. However THGEM is relatively easy to manufacture and handle and large sizes can be envisaged. Another advantage of using THGEM is that in case of any discharge happens inside the detector, the detector repair would be much easier as only the THGEM layer should be changed. Due to the structure, cascading THGEMs is possible to achieve higher gain at lower THGEM voltage, which decreases the discharge probability (Chechik et al, 2006; Breskin et al, 2010).

Due to its favorable characteristics, further studies and advances on THGEM and its applications have been carried out. Shalem et al reported advances in THGEM based gas detectors operating at atmospheric pressure (Shalem et al, 2006a) and low pressure (Shalem et al, 2006b). Within the same group, Cortesi et al developed an imaging THGEM-based detector successfully (Cortesi et al, 2006).

The McMaster group has been focusing on advanced radiation detector and nuclear instrumentation developments since 2007. The first simulation and test of THGEM-based detector for microdosimetry was carried out by Byun et al (Byun et al, 2009). They used Maxwell 2D software and GARFIELD code (Veenhof, 1998) to study the THGEM electric field configuration, the electron drift and avalanche process as is shown in Fig. 1.2.

The prototype detector was developed by Orchard (see Fig. 1.3) (Orchard, 2010; Orchard et al, 2011). The overall performance of the detector in a mixed neutron and gamma-ray field was investigated. It was shown that Orchard's detector generated the expected signal. Concurrently, Hanu et al carried out a Monte Carlo simulation of the detector neutron response (Hanu et al, 2010). Both neutron energy and angular responses were computed for various neutron beam conditions. It was shown that the energy response 
was consistent with the reported experimental microdosimetric spectra. Moreover, a THGEM X-ray imaging detector was successfully developed in 2013 (Hanu, 2013; Hanu et al, 2015).

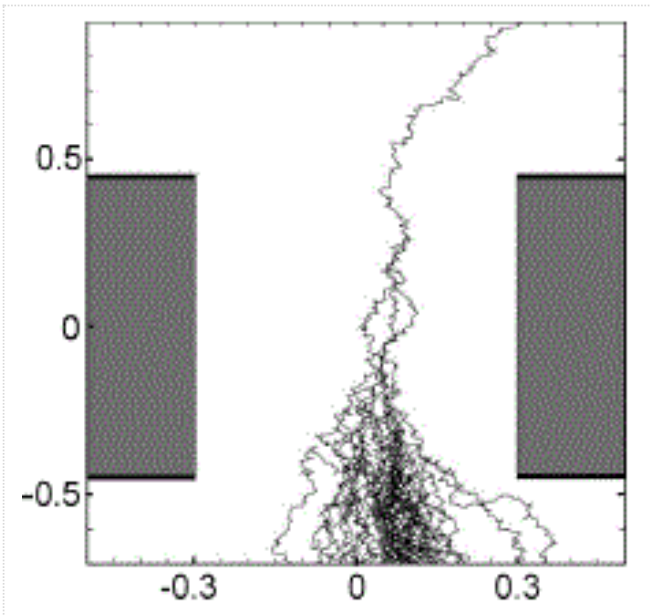

Fig. 1.2 Simulated electron avalanche process for a THGEM with $0.9 \mathrm{~mm}$ thickness and $0.6 \mathrm{~mm}$ hole diameter and $1200 \mathrm{~V}$ bias voltage (Byun et al, 2009)

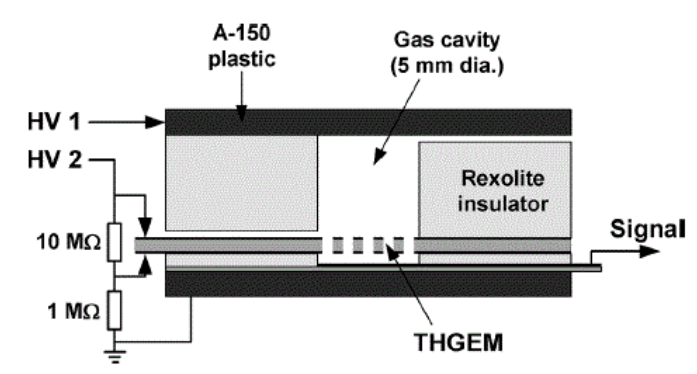

a.

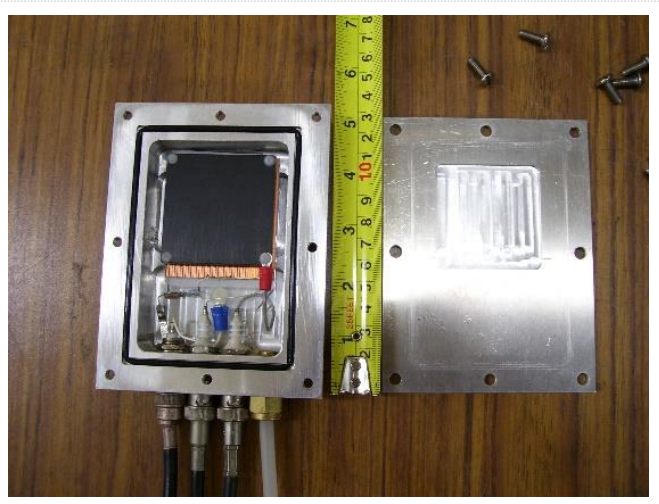

b.

Fig. 1.3 a. Conceptual drawing of the prototype THGEM detector (Byun et al, 2009), b. constructed and assembled prototype single volume THGEM-based microdosimetric detector (Orchard et al, 2011) 


\subsection{Motivation, Goal and Approach of the Thesis}

Neutron fields are mostly man-made, and are encountered near nuclear fission reactors, storage areas of highly radioactive waste and near particle accelerators, which are used for research, medical, and industrial applications. At high altitudes due to high-energy cosmic rays are where natural neutron fields are present. In this case neutrons are responsible for the most of the radiation by which the aircraft and their air crews are exposed (Waker et al, 2002; Bramlitt and Shonka, 2015; Braby, 2015). Hence, in all these cases neutron dosimetry is an important issue and the microdosimetric information of neutrons is vital in radiation protection and radiotherapy. (Van Eijk, 2004)

Although generally considered the best available detectors, the conventional TEPCs which are commercially available, have some shortcomings and hence do not always provide satisfactory results in mixed neutron-gamma fields. In weak radiation fields, where there is low dose rate, due to its low neutron detection efficiency and insensitivity to epithermal neutrons, using a conventional TEPC is impractical. Many studies have been performed to increase the sensitivity to neutrons by basically increasing the surface area of the gaseous sensitive volume by incorporating a large number of miniaturized TEPCs and combining all inputs to provide a single output. This was the concept of the so called multielement or multi-cellular TEPC. (Kliauga, 1989; Boutruche et al, 1994; Bordy et al, 1995; Waker and Aslam, 2010; Waker et al, 2011)

On the other hand, in the presence of intense mixed neutron and gamma-ray field, the dose rate is too high to be processed by the relatively large size conventional TEPC and chain of electronics, due to the severe pulse pileup and high dead time (Qashua, 2010). Hence there is a need to miniaturize the size of the detector (Burmeister et al, 2001; De Nardo et al, 2004). However, following the conventional method of electron multiplication using wire electrodes for the small size multi-element detector is extremely difficult, time consuming and quite expensive. Instead, using THGEM for electron multiplication process eliminates the need for multiple anode wires while simplifying the overall cost and fabrication of a TEPC particularly the two-dimensional detector. 
Hence, an advanced two-dimensional microdosimetric detector based on the THGEM technology was proposed for the subject of this thesis. The main objective of this work was to design, construct and implement a 2D microdosimetric TEPC using the THGEM technology to effectively measure the spatial distributions of high and low linear energy transfer radiation doses simultaneously in mixed radiation fields within a single measurement which will enable us to overcome the operational limitation of the classical TEPCs, particularly for high dose rate fields.

The main feature of the device is that it consists of multiple individual TEPCs (array of $3 \times 3$ or $5 \times 5$ or more gas cavities) within a single chamber, which accordingly requires multiple $(9,25$ or more) pulse height analyzers to process each individual signal simultaneously. This requires 9, 25 or more electronic components, such as preamplifier and digital signal processor (DSP) to process each signal individually, which is very expensive, complex and causes difficulty for the portability of the system. This issue was resolved by developing a custom made multi-input digital signal processing system using a modern microcontroller interfaced with an ADC at comparatively low cost.

This dissertation is focused on an approach towards the goal of explaining the design, construction and implementation of the two-dimensional detector based on the THGEM. In chapter 2, the detector design and construction are explained in detail. Chapter 3 presents the development of a McMaster Multi-input digital signal processing system (MMI-DSP) and the related benchmark test results. Experimental methodology and the neutron irradiation facility are introduced in chapter 4 . The details of data analyses methodology are described in chapter 5 . The main focus of chapter 6 is to present the comprehensive dose measurement of the ${ }^{7} \operatorname{Li}(\mathrm{p}, \mathrm{n})$ McMaster accelerator mixed radiation neutron and gamma-ray field using standard spherical 1/2" TEPC to effectively characterized the Tandetron Accelerator radiation field and hand irradiation facility which was used as the neutron source for this study. Chapter 7 presents and discusses the results for a single and an array of $3 \times 3$ gas cavities THGEM detector. Ultimately, the thesis will be completed with the conclusion and proposed potential future work in Chapter 8. 


\section{Chapter 2}

\section{Detector Design and Construction}

2.1 Detector chamber

2.2 Cathode

2.3 Sensitive Volume

2.4 Thick Gas Electron Multiplier (THGEM)

2.5 Readout Board

2.6 High Voltage Divider

2.7 Detector Wiring and Assembly

2.7.1 Single Gaseous Cavity

2.7.2 2D Multi-volume (Array of $3 \times 3$ Gaseous Cavities)

2.8 Smaller Sensitive Volumes

2.9 Vacuum Test and Gas filling 


\section{Detector Design and Construction}

The 2D THGEM detector consists of four layers enclosed in an Aluminum chamber with low pressure. In this chapter, the design of each layer together with the choice of material and fabrication process will be explained in detail.

\subsection{Detector Chamber}

The first essential step in the design of the 2D THGEM detector is to choose the geometry and the range of operating pressure. Fig. $2.1 a$ and $b$ show the geometry and design of the chamber lid and baseplate. The chamber consists of two parts, the baseplate which is the main body that the detector layers will be mounted on and the covering lid.

The baseplate was designed in a way that four different sizes of detector $(5 \times 5 \mathrm{~cm}, 6 \times 6 \mathrm{~cm}, 9 \times 9 \mathrm{~cm}$ and $12 \times 12 \mathrm{~cm})$ can be mounted on it. The outer diameter is $25 \mathrm{~cm}$ and the inner diameter is $20.6 \mathrm{~cm}$. There are two threaded holes for $3 / 8 " \mathrm{NTP}$ connectors (SHV), devised for high voltage connectors. There is a threaded hole for 1/4" NTP connectors, devised for a gas valve. A $4.1 \mathrm{~cm}$ diameter hole is machined for the multipin feed through. All the HV, signal and gas valve connectors used for the chamber are suitable for vacuum applications. There are 16 taps for 4-40 screws, devised for mounting detector layers with various sizes.

The covering lid is an empty cylinder (20.6 cm in diameter and $5.8 \mathrm{~cm}$ height), having a groove for placing a 1/8" (AS568B) Vitron-A O-ring for the best sealing result. For closing and sealing the chamber, 14 holes for $3 / 8 " \times 16-1$ bolts are machined around.

The detector chamber has been fabricated from 6061 Aluminum alloy at the McMaster engineering machine shop in accordance with the mentioned design. After fabrication, in order to get rid of oil residues due to machining, both parts were cleaned with ethanol. High voltage and gas valve connectors were hermetically sealed to the chamber body using low outgassing epoxy (Loctite 1C Hysol). A Multi-pin feedthrough was installed according to the drawing shown in Fig. 2.2, using a centring ring, O-ring and KF-40 flange. Fig. 2.3 illustrates the final fabricated chamber lid and baseplate. 

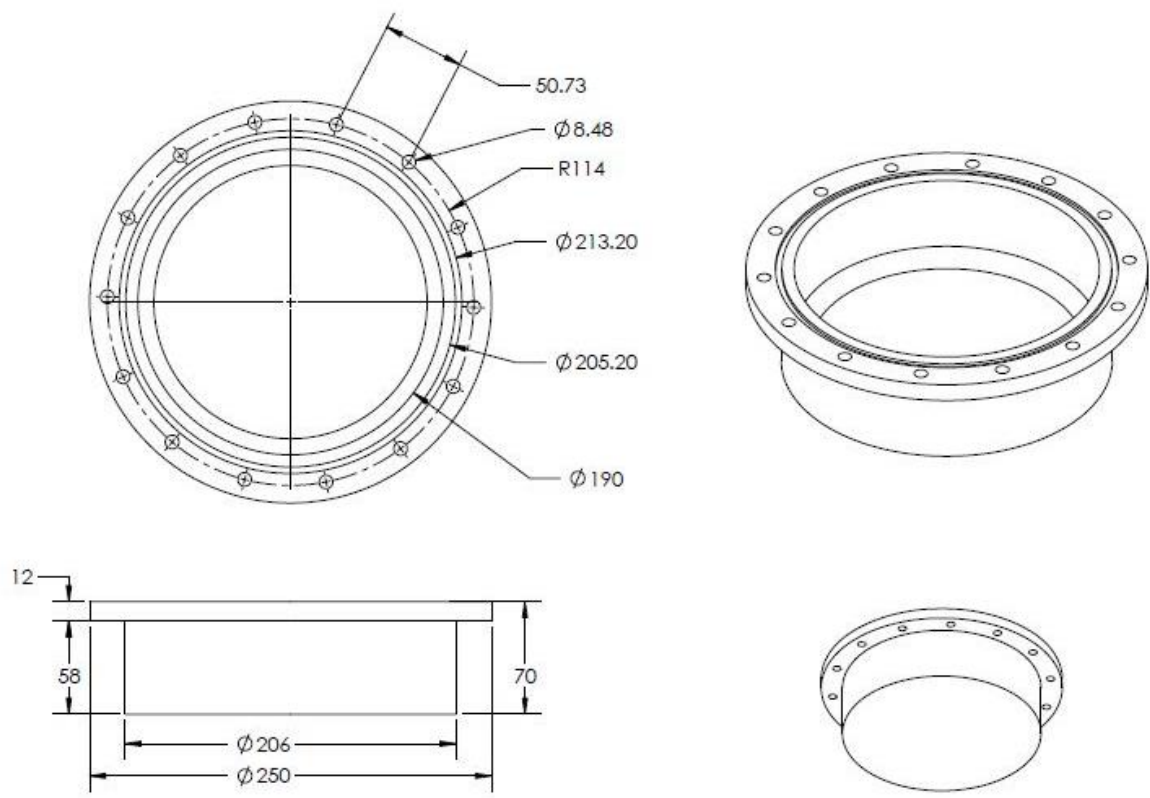

a.
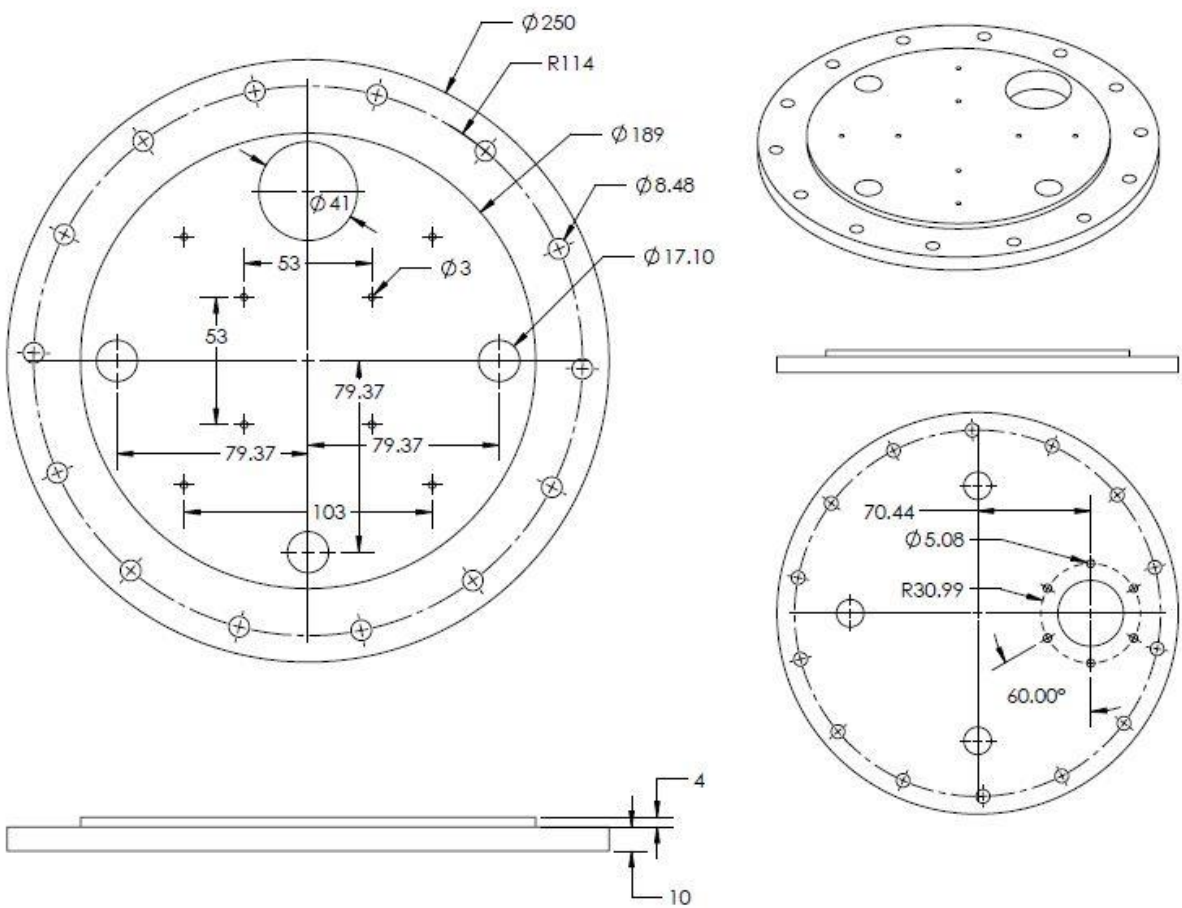

b.

Fig. 2.1 a. Detector chamber lid design, b. Detector chamber baseplate design. Note: All the dimensions are in millimetre 


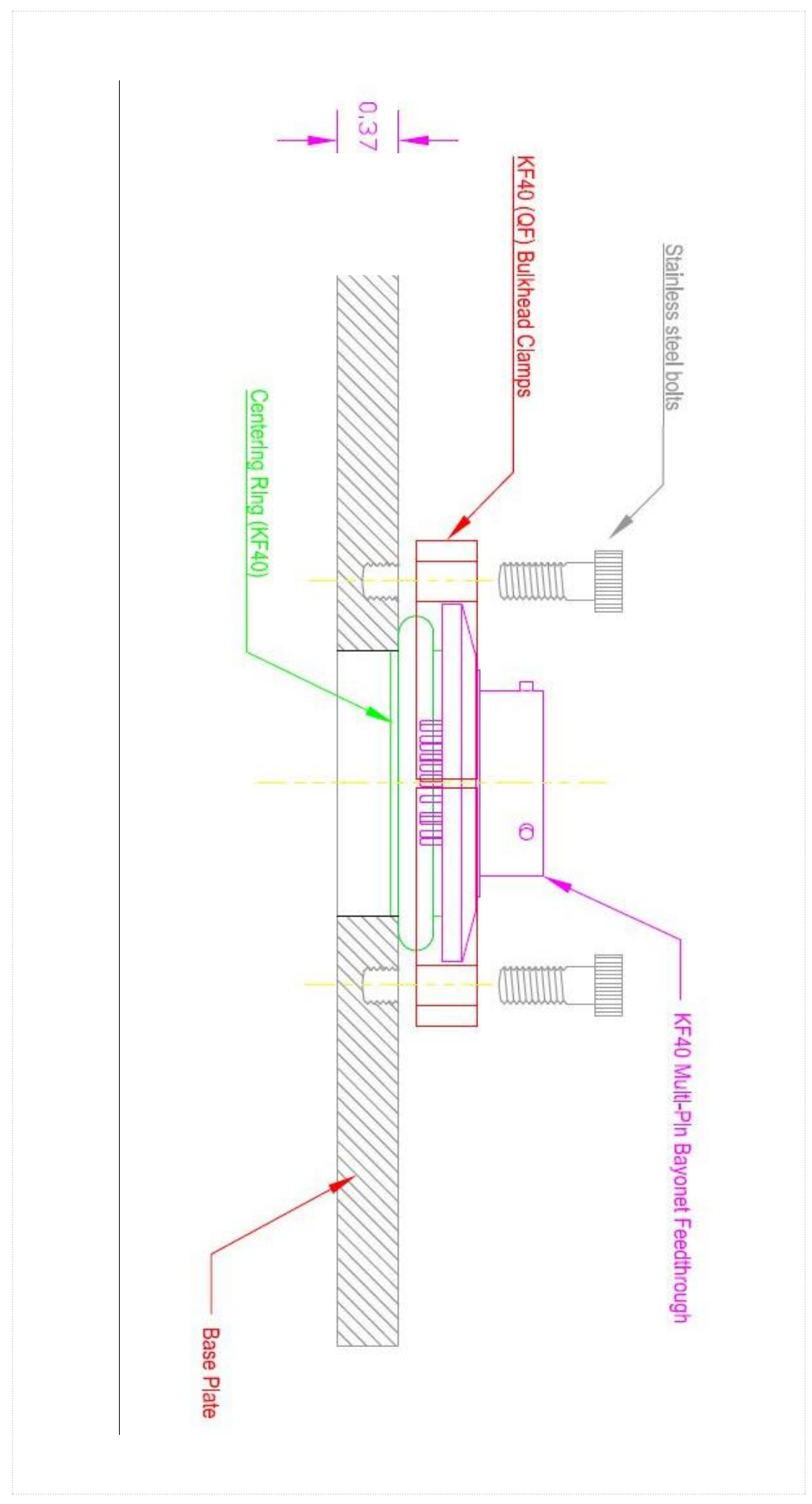

Fig. 2.2 Multi-pin installation on the detector chamber baseplate 


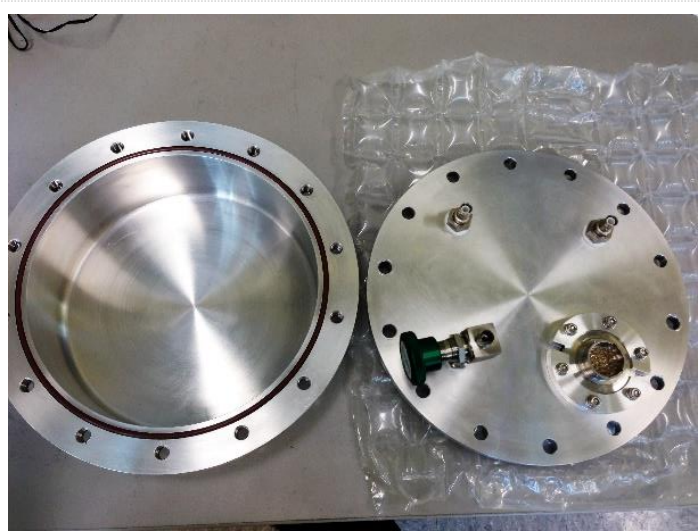

a.

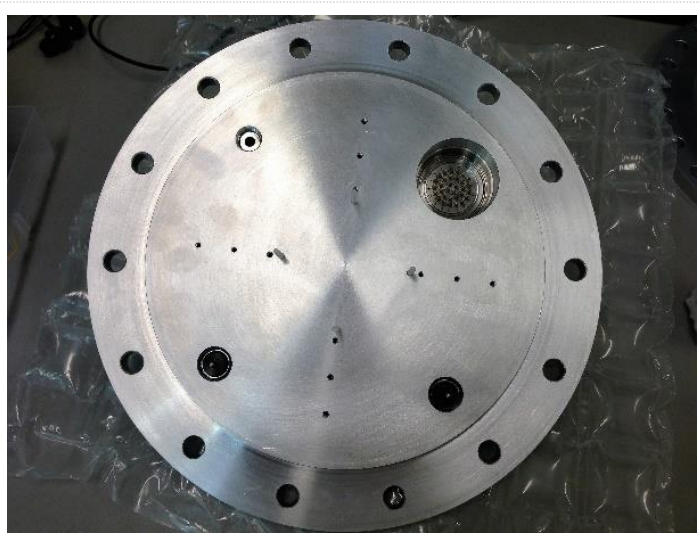

b.

Fig. 2.3 a. Fabricated detector chamber lid and air side of the baseplate, b. Fabricated detector chamber baseplate (vacuum side)

A simpler design was devised for the baseplate of the chamber for the preliminary detector response tests. In this design, the multi-pin connector was replaced by three BNC connectors for the signal. This was done to reduce the unknown factors as much as possible and just test if the detector components were working. After the preliminary tests were done and the single detector response was investigated, the baseplate was replaced by the original design with the multi-pin connector to test all 9 detectors simultaneously. The simpler baseplate design is shown in Fig. 2.4 and 2.5.

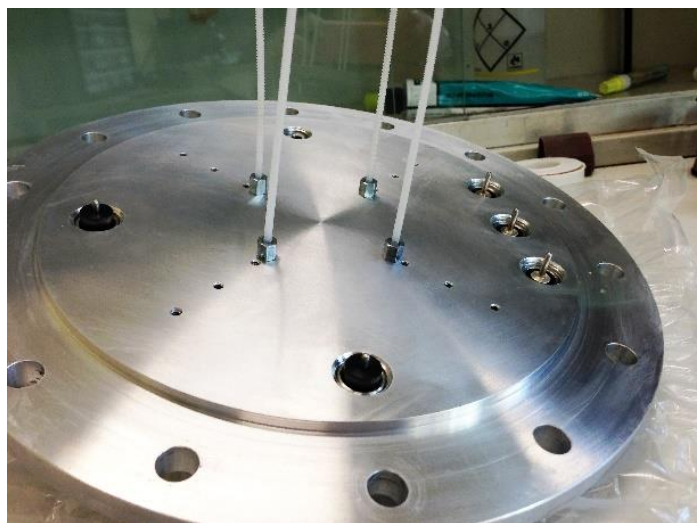

a.

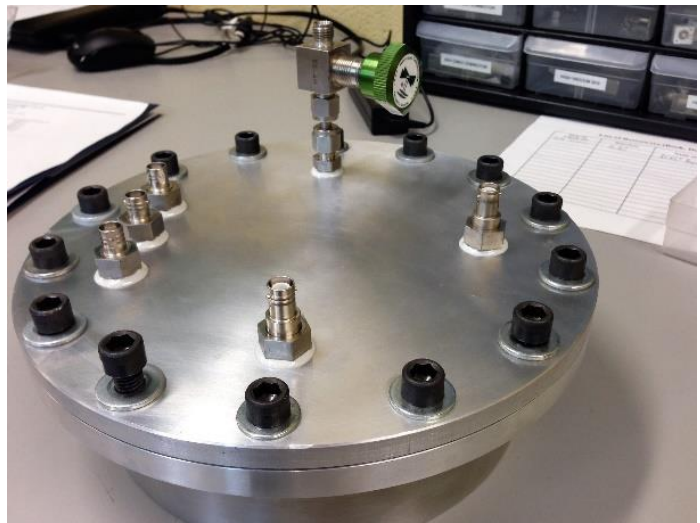

b.

Fig. 2.4 a. Fabricated detector chamber baseplate with 3 signal inputs (vacuum side),

b. Assembled detector chamber with 3 signal inputs 


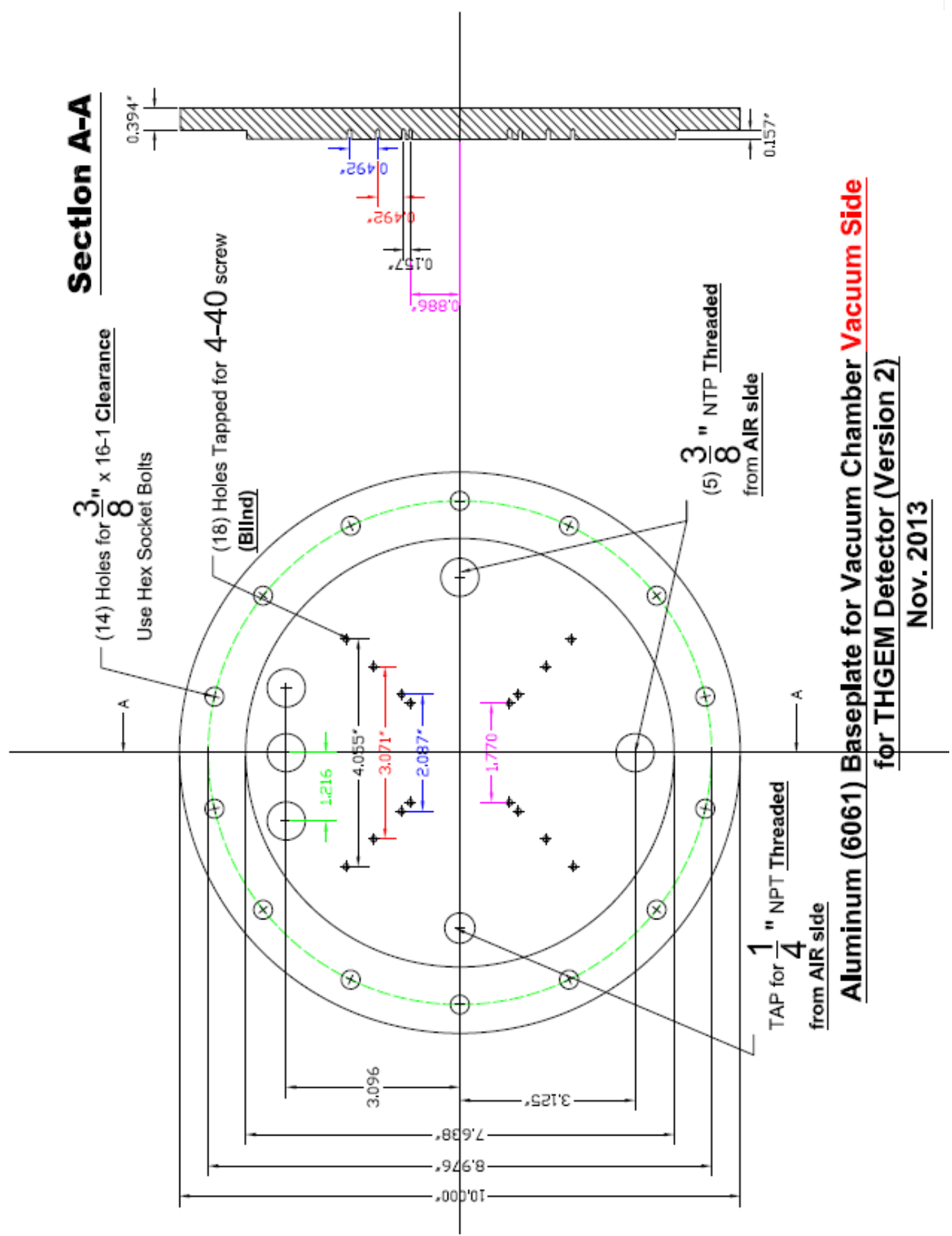

Fig. 2.5 Detector Chamber baseplate design with 3 signal inputs. Note: All dimensions are in inches. 


\subsection{Cathode}

To fulfil the requirement of utilizing both tissue equivalent and conducting material, A-150 plastic was used as the cathode and was grounded. This conducting plastic, originally developed by Shonka (1958), is a mixture of calcium fluoride, polyethylene, nylon, and carbon as a conductive replacement for Oxygen. The A-150 layer is $2 \mathrm{~mm}$ thick and $6 \times 6 \mathrm{~cm}^{2}$ in dimension. The design and fabricated cathode layer are shown in Fig. 2.6.

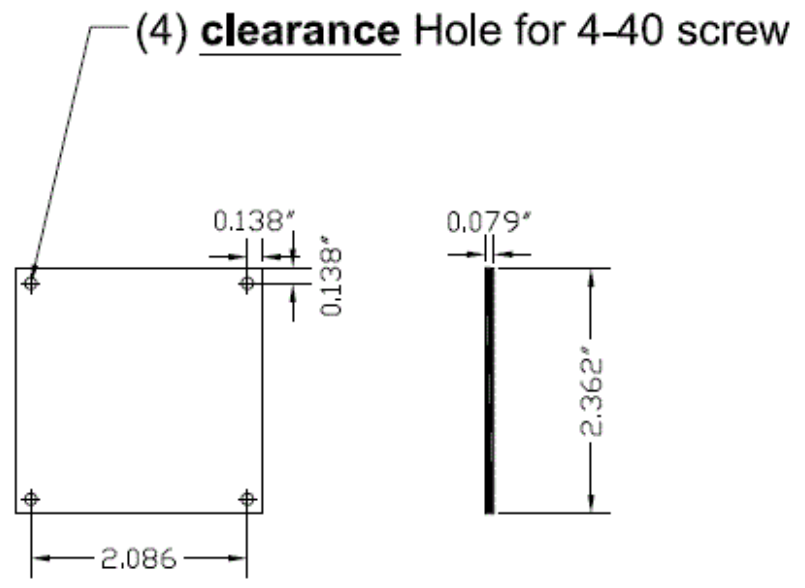

a.

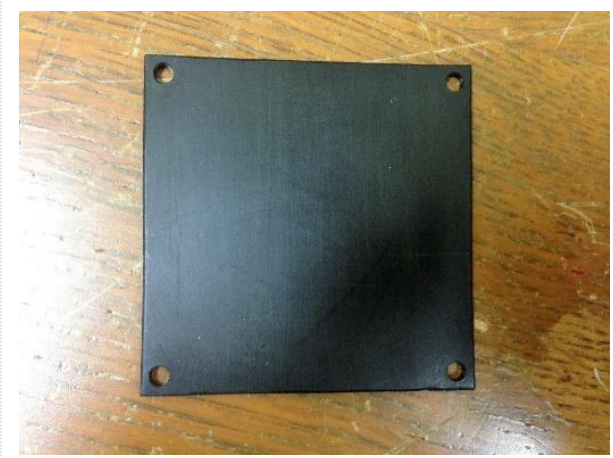

b.

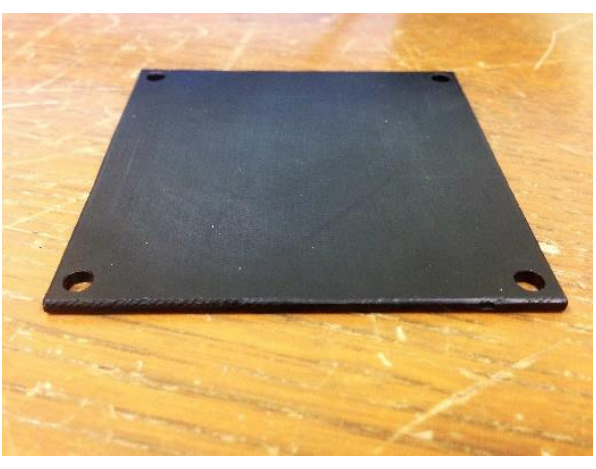

c.

Fig. 2.6 A-150 layer as a cathode a. Designed, b \& c. Fabricated. Note: All the units are in inches 


\subsection{Sensitive Volume}

The next layer which contains the actual sensitive volumes of the detector is shown in Fig. 2.7. Rexolite 1422 (hereafter Rexolite), cross-linked polystyrene (C-LEC Plastic Inc.), was chosen as an insulating tissue equivalent material, in which 9 right cylinders were machined as gas sensitive volumes. This layer is $5 \mathrm{~mm}$ thick and $6 \times 6 \mathrm{~cm}^{2}$ in dimension. There are 9 right cylinders $5 \mathrm{~mm}$ in diameter and $5 \mathrm{~mm}$ in height. For the purpose of gas filling which will be explained later in this chapter, $1.2 \mathrm{~mm}$ deep grooves were machined across the layer and holes.
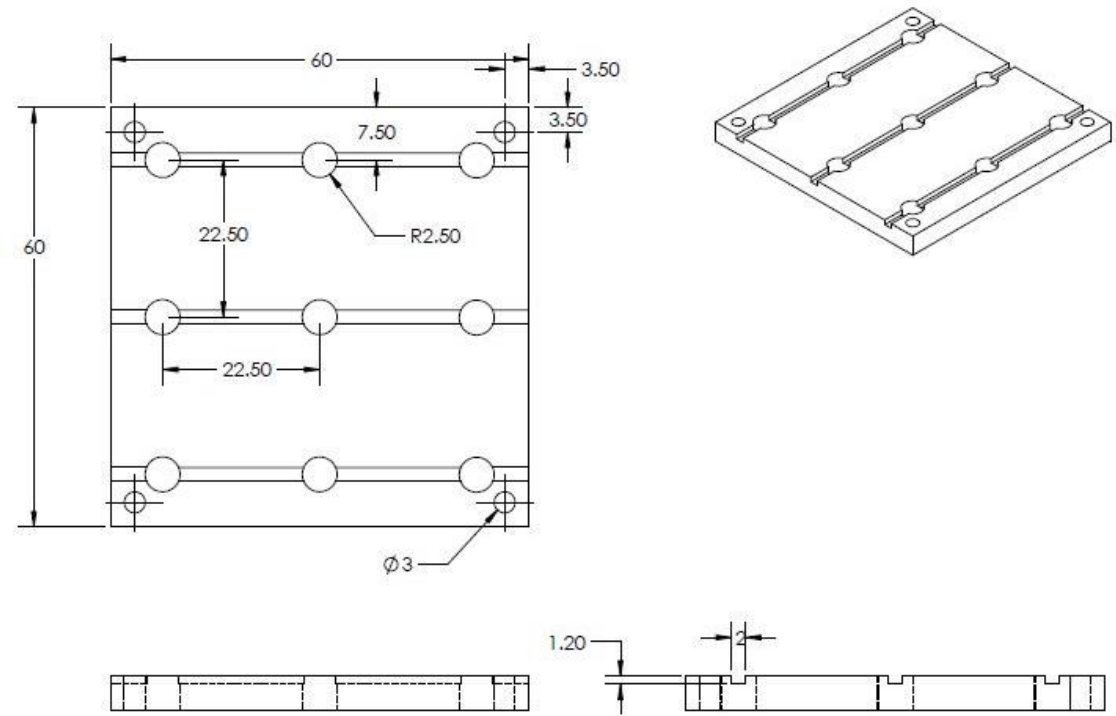

a.

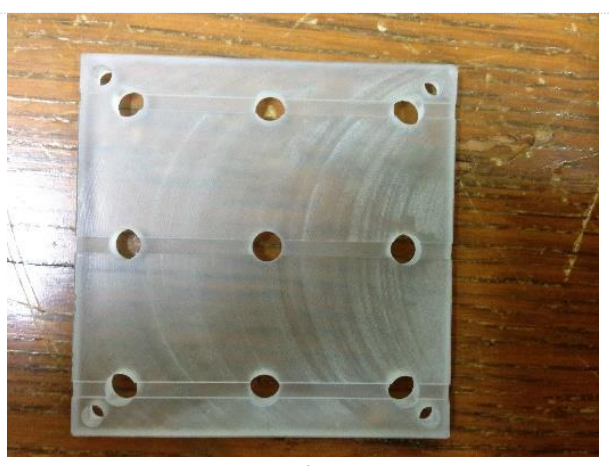

b.

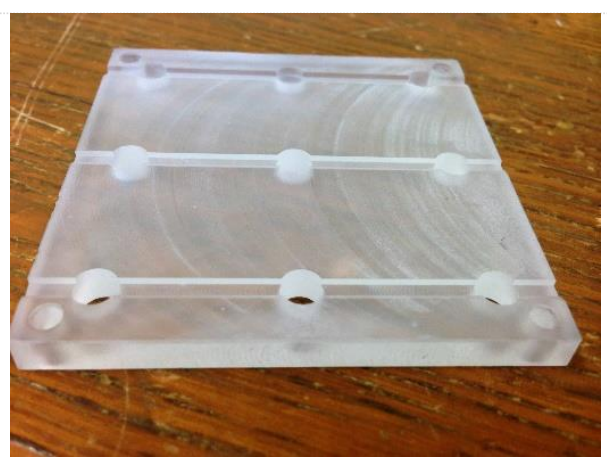

c.

Fig. 2.7 Rexolite layer as sensitive volume a. designed, b \& c. fabricated. Note: All the units are in mm 


\subsection{THGEM}

The first THGEM used for a prototype micro dosimetry detector at McMaster University was manually micro-drilled and chemically etched and cleaned in our lab (Orchard, 2010). The maximum number of holes was 32 with $0.35 \mathrm{~mm} \pm 0.01 \mathrm{~mm}$ diameter hole, the pitch of $0.64 \mathrm{~mm} \pm 0.01$ and varying insulator thickness. The results were successful and promising, however, fabrication of a greater number of holes, especially in the present case of more than 4000 holes, with such a manual technique is impractical. Thus industrial manufacturing was adopted for faster and more efficient and accurate THGEM fabrication. To this end, first, the THGEM had to be designed using a printed circuit board (PCB) software. Altium Designer which is one of the powerful software was used to design the THGEM layer. Basically, the THGEM consists of FR4 glass-reinforced epoxy laminate sheet coated with 50-micron copper on both sides. The overall thickness of THGEM is $0.41 \mathrm{~mm}$ with the outside dimension of $60 \times 60 \mathrm{~mm}$ and has $4 \times 3 \mathrm{~mm}$ copper pads on both sides allowing biasing bottom and the top layer of THGEM.

Fabrication of many holes is accomplished via computer numerical controlled (CNC) drilling machines at the industrial site. Hence fabrication of THGEM with any shapes or sizes is achievable. The most substantial challenge in the fabrication process is to drill all holes uniformly clean. Any protruding, ragged sharp edges remaining on the copper surrounding the holes can result in extremely high electric field gradients which can destroy the THGEM during a highly ionizing event (see Fig. $2.8 f$ ). To avoid this over a large number of holes, frequent tooling changes are mandatory.

The THGEMs used in this work were ordered via two different manufacturers. First sets (hereafter old THGEM) were ordered from a Chinese company via the online service offered through MyroPCB (www.myropcb.com) (see Fig. 2.8 a). In this case, we did not have any control on quality of fabrication, as it was just online ordering without having access to communicate with any technical support engineers. The second sets of THGEMs (hereafter New THGEM) were ordered from Milplex circuit Canada Inc., an expert printed circuit board (PCB) manufacturer (see Fig. 2.8 b). For improvement, we asked for more polishing and deburring process steps. Their process sequence is as below: 
1) Drilling: computer numerical controlled (CNC) drilling machines

2) Deburring: a conveyorized brushing machine equipped with 500 grit high speed rotating brushes.

3) Polishing with pumice: a conveyorized jet pumice that pumps a slurry of pumice and water from top and bottom at high pressure, on material passed along the conveyor.

4) Inspection: under a microscope for the edge of holes and deep scratches.

5) Micro-etching: a conveyorized chemical spray system. The chemical "tinlead stripper" contains approximately $10 \%$ Nitric Acid at approximately $110{ }^{\circ} \mathrm{F}$. This is repeated 3 times. A round of polishing with pumice and deburring is conducted following each round of micro-etching.

6) Final inspection under a microscope for the edge of holes to assure holes quality.

Fig. $2.8 c$ shows THGEM holes within a $5 \mathrm{~mm}$ sensitive volume under the microscope. THGEMs were inspected thoroughly each time before installation to pick up a clean, uniform THGEM without any sharp edges. This results in circumventing discharge and spark once raising applied high voltage.

For the new THGEMs, it was observed that there was a layer of dust on both sides, which is illustrated in Fig. $2.8 \mathrm{~g}$ and $h$. Thus all THGEMs were blown with high purity dry nitrogen gas to eliminate any dust and residue on the surface.

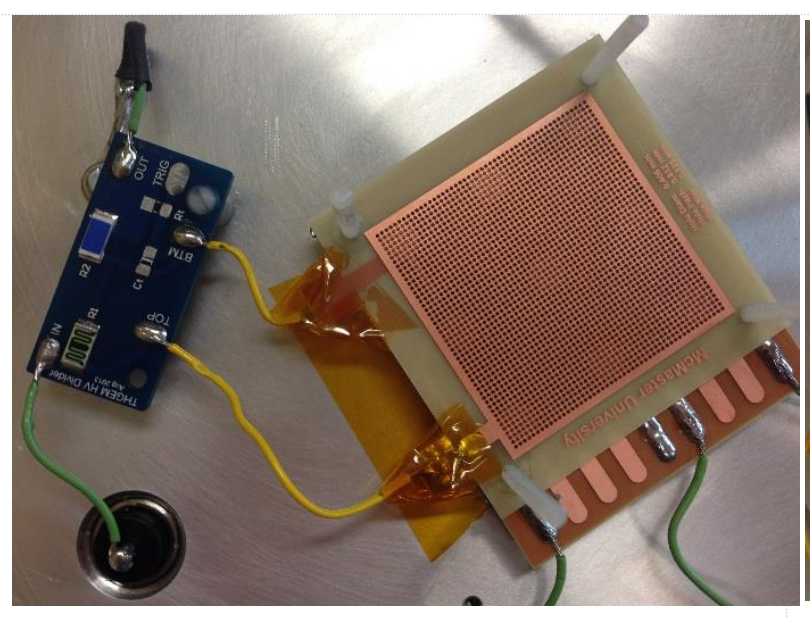

a.

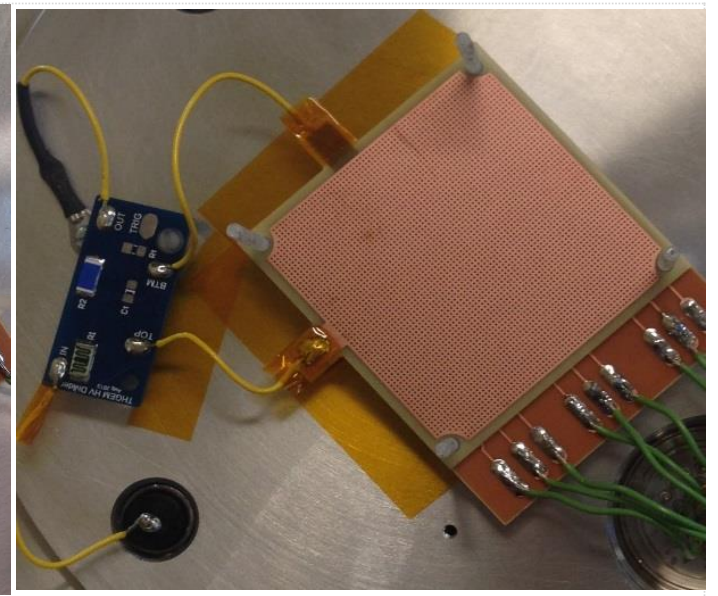

b. 


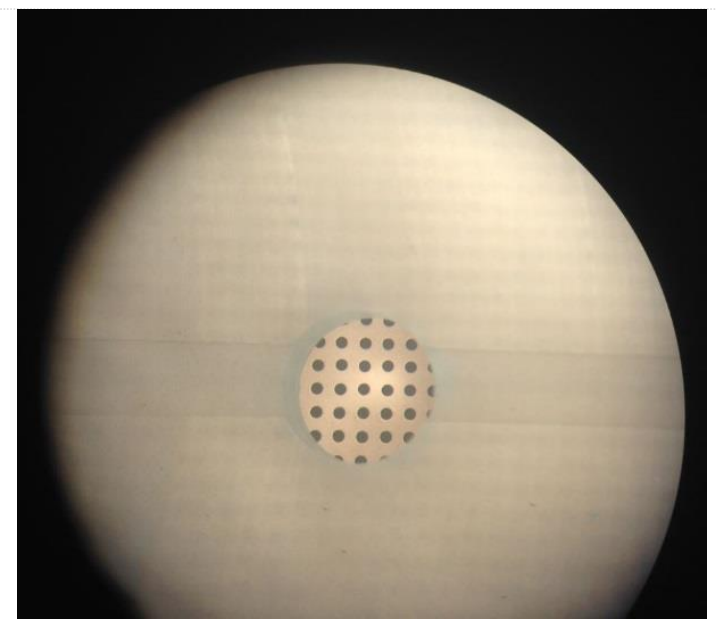

c.

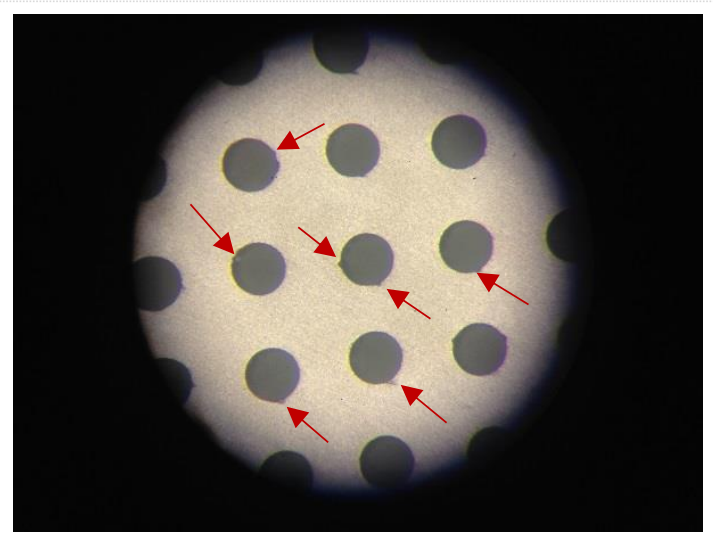

e.

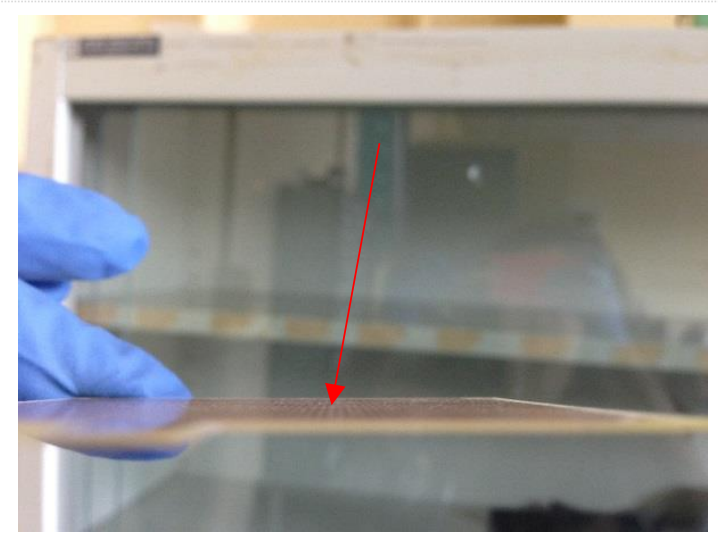

g.

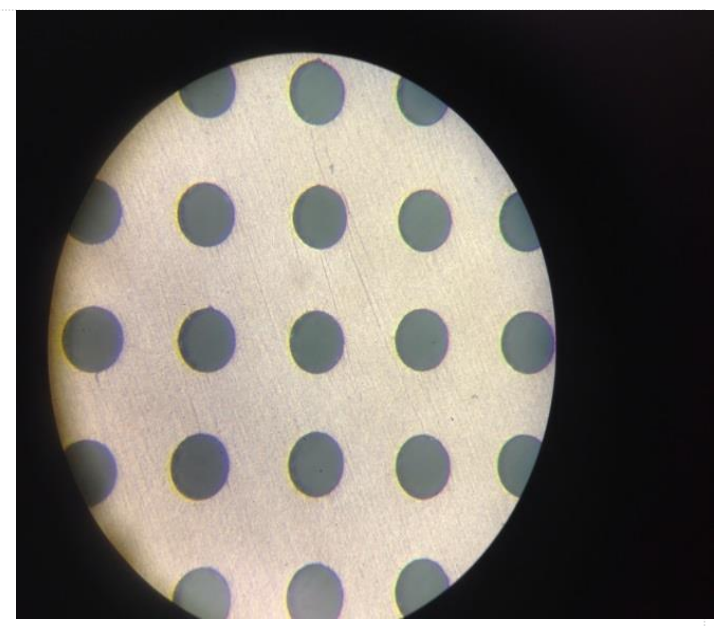

d.

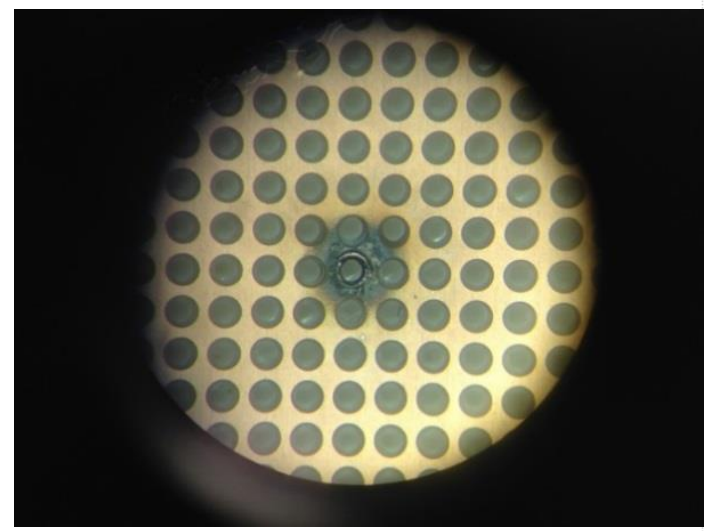

f.

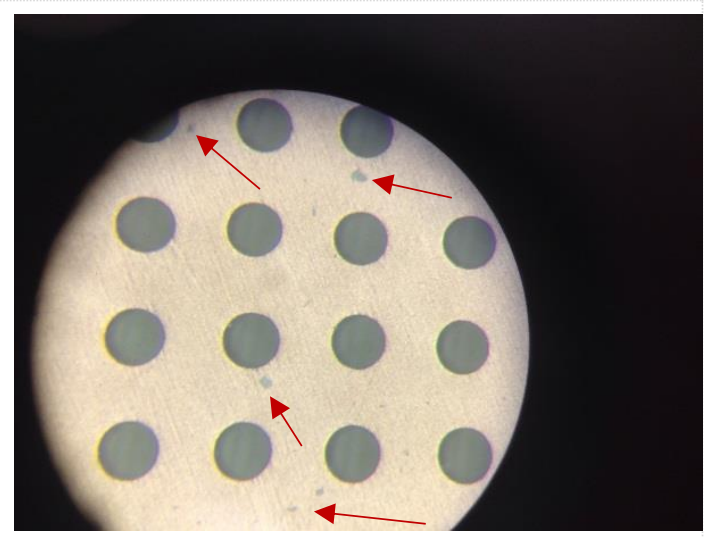

h.

Fig. 2.8 a. Old THGEM (fabricated by MyRoPCB Company, China), b. New THGEM (fabricated by Milplex Company, Canada), c. THGEM holes within a sensitive volume under a microscope, d. Typical acceptable clean and uniform THGEM holes, e. Typical imperfections of THGEM holes, f. Sparked induced damaged THGEM hole, g. A dusty layer on the surface of THGEM, h. Zoom in view of dust on the surface under microscope 


\subsection{Readout Board}

The Readout board was designed with a printed circuit board software, Altium Designer version 2.1 (Altium Ltd.). This board consists of an FR4 insulator coated with copper on both sides. The 2D pattern of 9 detectors as shown in Fig. 2.9 are etched for the collection of charges from each detector.
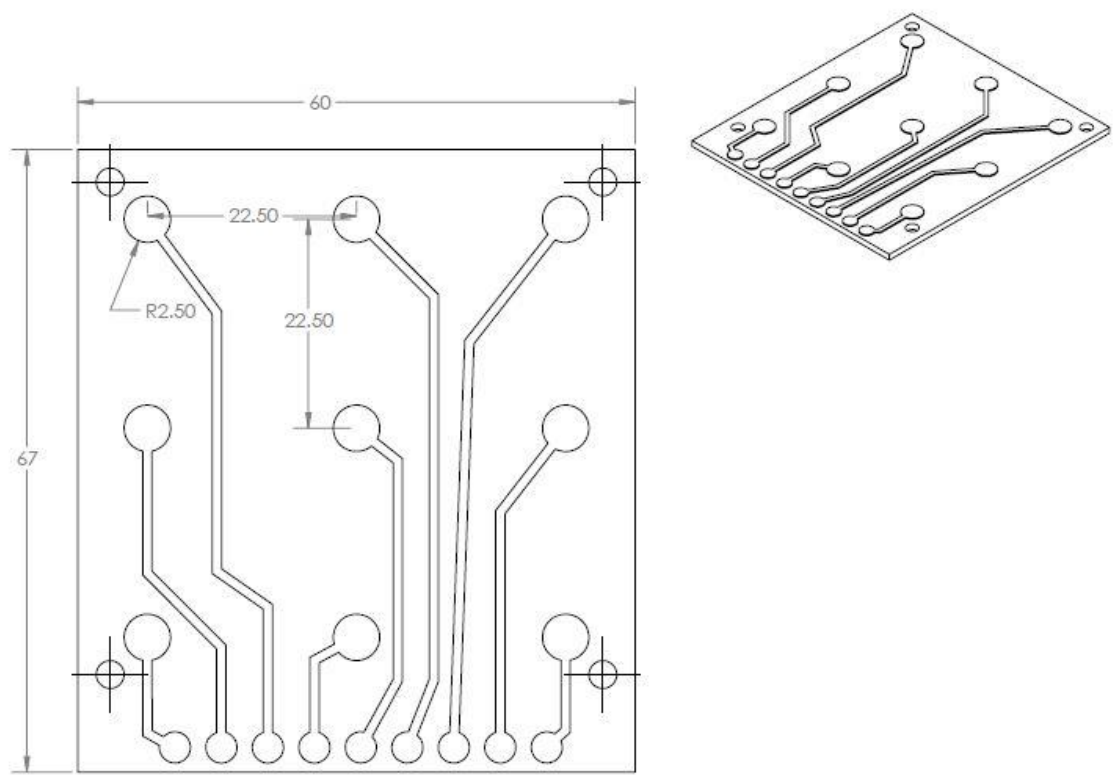

a.

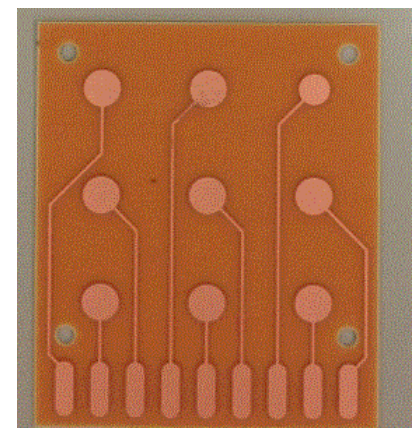

b.

Fig. 2.9 Readout board layer a. designed, b. fabricated. Note: All the units are in mm. 


\subsection{High Voltage Divider}

In order to bias THGEM and apply a high voltage to the bottom and top side of THGEM, a high voltage divider was designed (Fig. 2.10). It is a printed circuit board with two resistors of $R_{1}=10 \mathrm{M} \Omega$ and $R_{2}=2 \mathrm{M} \Omega$, dividing the input high voltage between the bottom and top layer of THGEM such that 5/6 of input high voltage would be applied across THGEM and the 1/6 remainder would be between bottom of THGEM and collection plate (see eq. 2-1 and 2-2)

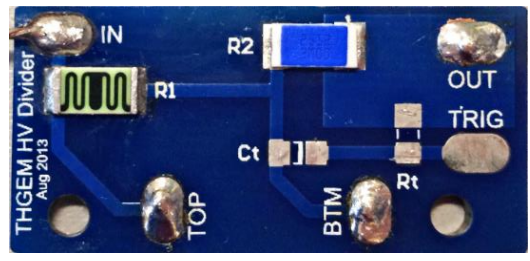

Fig. 2.10 HV divider board

The Resistors $R_{1}$ and $R_{2}$ are chosen in such a way that the majority of $\mathrm{HV}$ is applied across the THGEM and the remainder is to direct the electrons to the collection plate (CP).

$$
\begin{gathered}
\Delta V_{T H G E M}=\frac{V 1_{\text {in }} \times R_{1}}{\left(R_{1}+R_{2}\right)} \\
\Delta V_{C P}=\frac{V 1_{\text {in }} \times R_{2}}{\left(R_{1}+R_{2}\right)}
\end{gathered}
$$

where $V l_{\text {in }}$ is the input applied a high voltage to the THGEM BNC input on the detector. 


\subsection{Detector Wiring and Assembly}

After fabrication of the Aluminum chamber and installing the high voltage, signal and gas valve connectors on the plate, the detector was sealed and closed for a vacuum test. Connecting to an oil diffusion pump, the chamber was pumped down to $10^{-4}$ torr for 2 days and no leakage was observed, indicating the chamber was ready for detector assembly.

The final design of the assembled detector with a single THGEM layer is illustrated in an exploded view in Fig 2.11. Below the assembly of Single volume baseplate and 2D multi-volume baseplate will be explained.

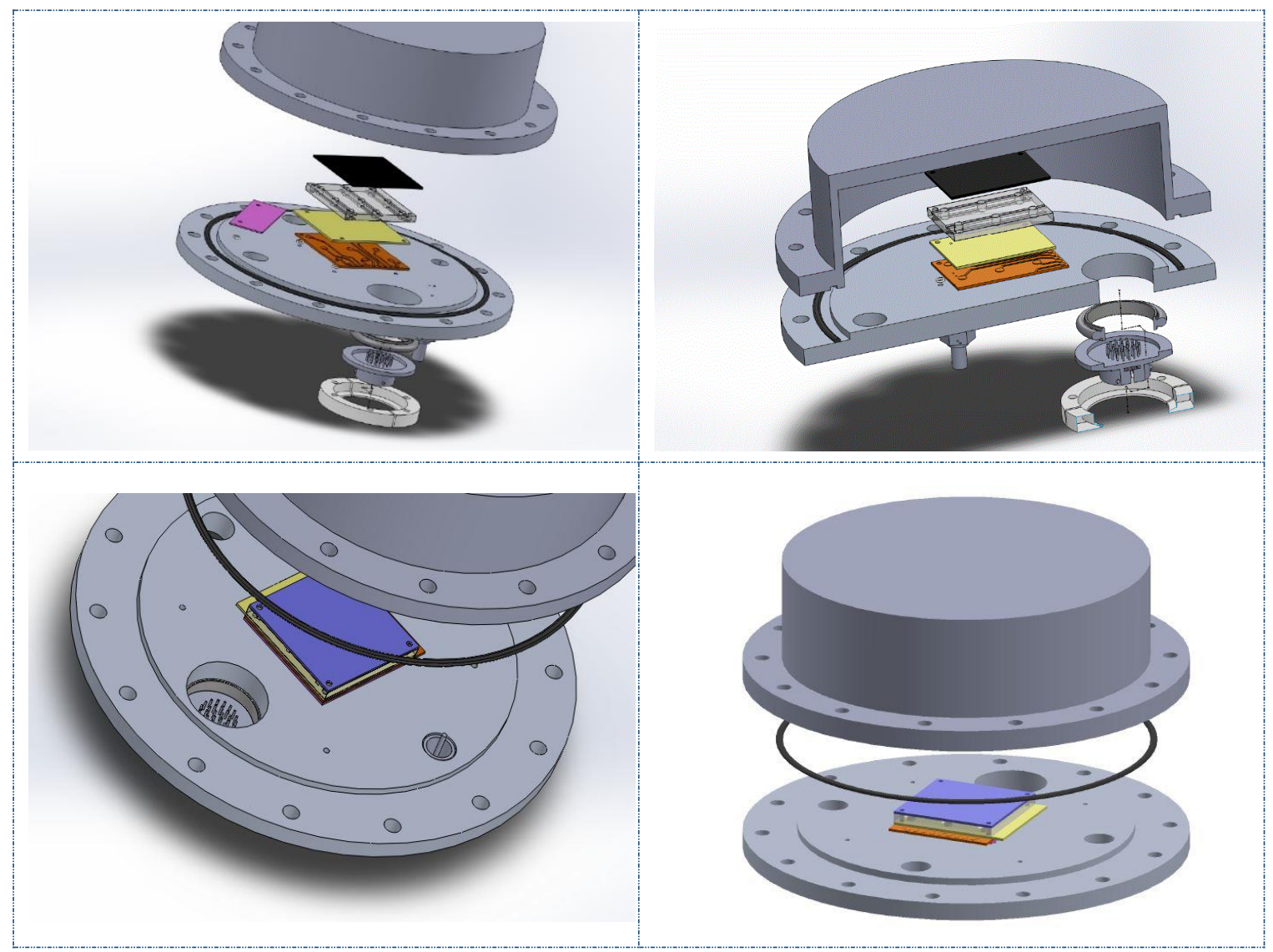

Fig. 2.11 Exploded view of the detector final design 


\subsubsection{Single volume detector configuration}

The bottom most layer is the readout board mounted on the surface of the Aluminium baseplate. For the single detector test, there are only three BNCs designated for signal on the baseplate, soldered to the three detector collection plate as shown in Fig. 2.13. Next layer is the THGEM mounted on top of the readout board with a gap of $1 \mathrm{~mm}$ made up of Teflon spacers. Using a HV divider, the top and bottom layer of THGEM will be biased from a SHV connector named THGEM HV (see Fig. 2.12). Rexolite layer as sensitive volumes is mounted directly on top of THGEM layer. Finally, A-150 layer as a cathode is mounted on top of Rexolite. A separate SHV connector is used to bias cathode individually.

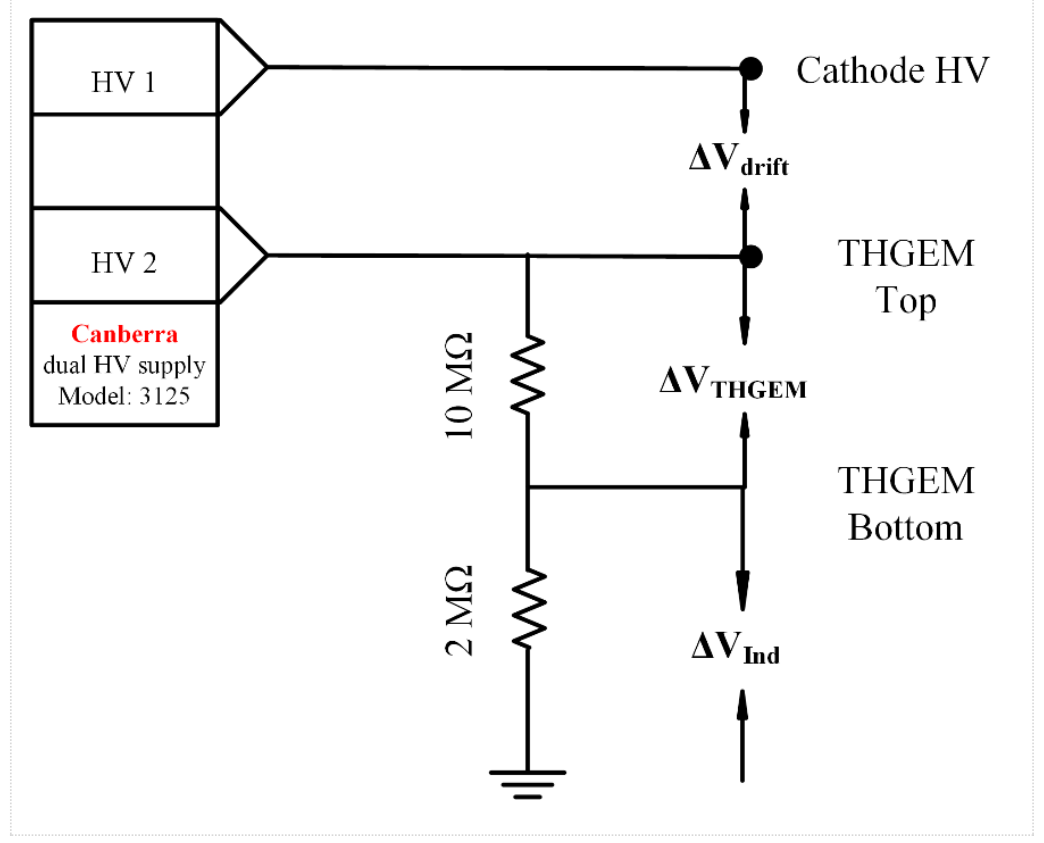

Fig. 2.12 High voltage connections circuit diagram for single THGEM layer arrangement 


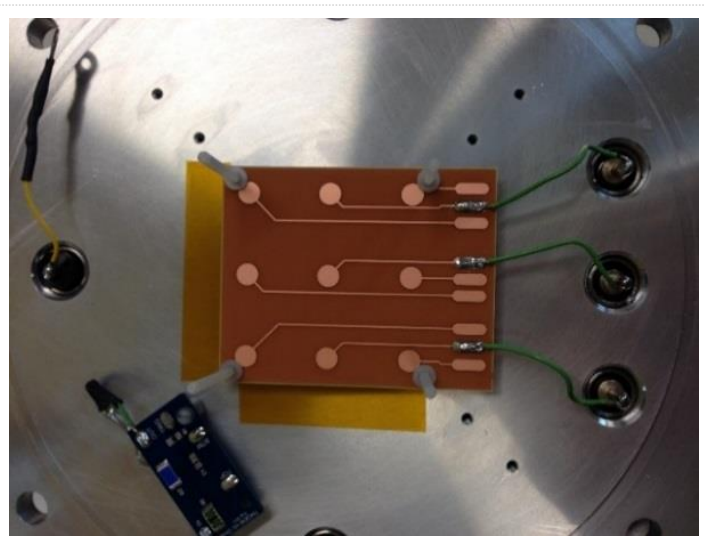

a.

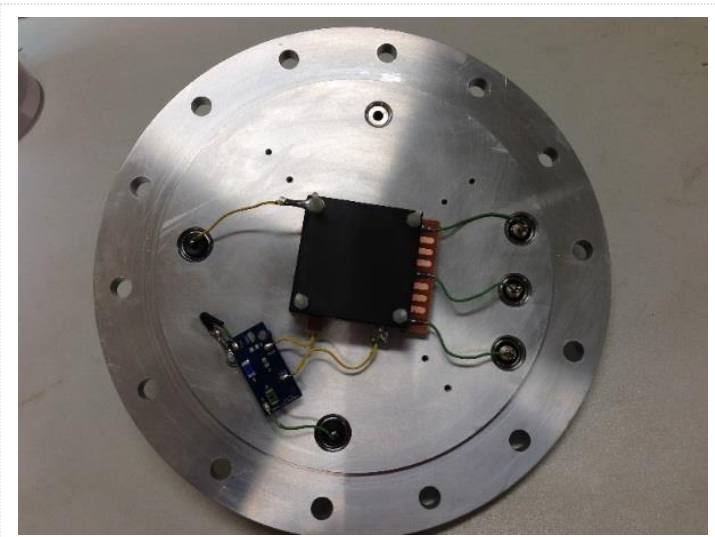

b.

Fig. 2.13 Single volume detector assembly, a. 3 BNCs soldered to readout board for signal collection, b. Final detector assembly

\subsubsection{2-Dimensional multi-volume}

After testing the detector and assuring the detector response and performance for a single detector, it is time to test 2 -dimensional multi-volume detector configuration. To this end, the new baseplate fabricated for multi-pin installation should be assembled and wired. All the layers are mounted the same as in the single detector configuration, except that all 9 channels of readout board layer have to be soldered to the 9 pins of the multi-pin feedthrough for collecting signals from all 9 detectors simultaneously.

The multi-pin instrument feedthrough (model: IFTRG327018B, Kurt J. Lesker Canada Inc.) has circular bayonet-locking connectors on the air-side and crimp pins on the vacuumside. As is illustrated in Fig. 2.2 and 2.11, the KF flange is sealed with a centring ring and O-ring combination making them high vacuum compatible. A bulkhead clamp is used for mating KF flanges to the bulkhead.

The airside crimp plug is assembled according to the manufacturer's instruction. For the wire assembly, first, one end of the RG-174 cable is stripped and soldered to the gold plated contact provided by the manufacturer. Using an insertion tool, the soldered contact is inserted into the plug. This process is repeated 9 times to have 9 individual signal cables for the 9 detectors. Each pin is named with a specific letter on both sides of plug and on the detector vacuum side, so tracking each detector would be possible. The other end of the 
cables is BNC connectors for connecting to the input of the preamplifier. All cables are labelled with the detector numbers to avoid any confusion or mistakes. Fig. 2.14 illustrates the detector assembly, multi-pin plug, and all required cables.

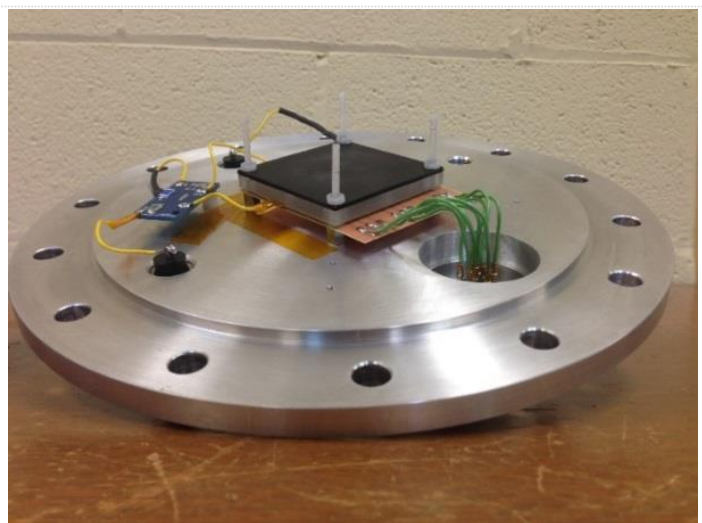

a.

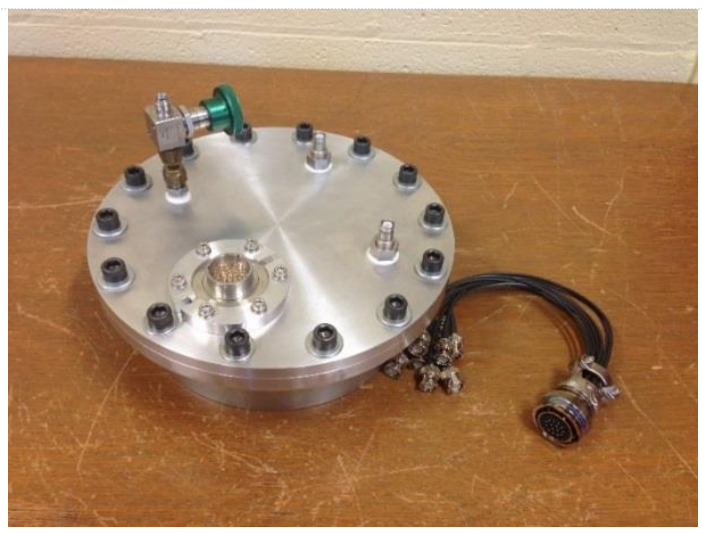

C.

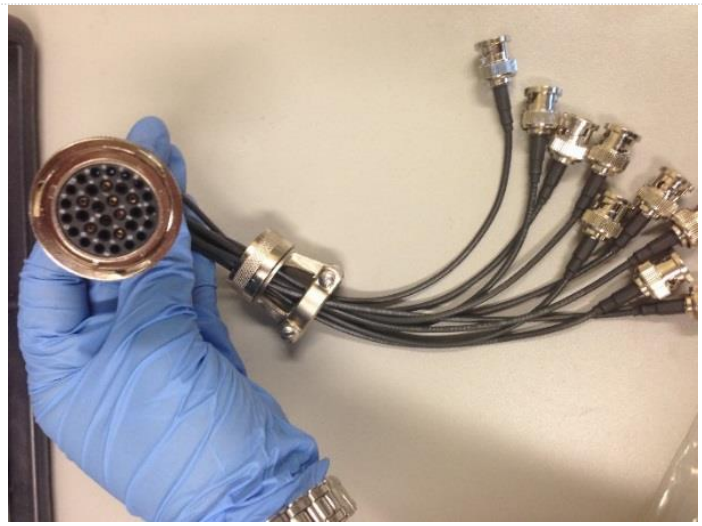

e.

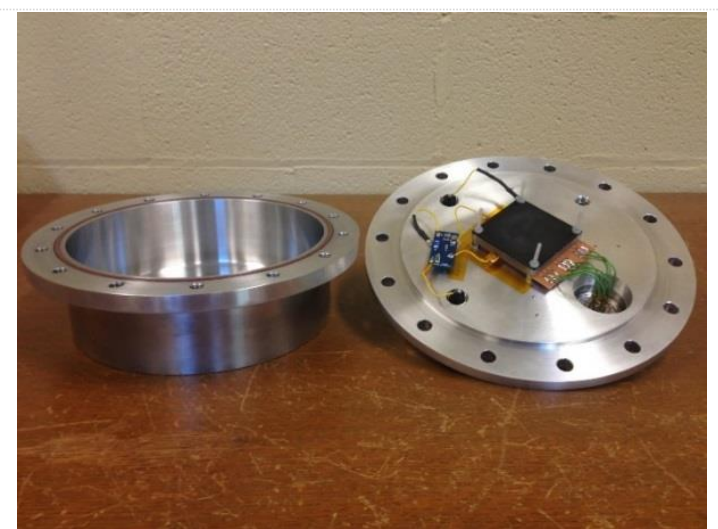

b.

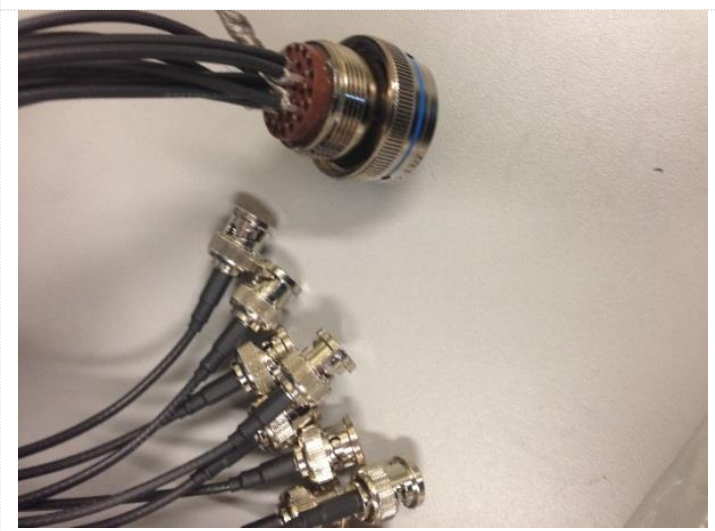

d.

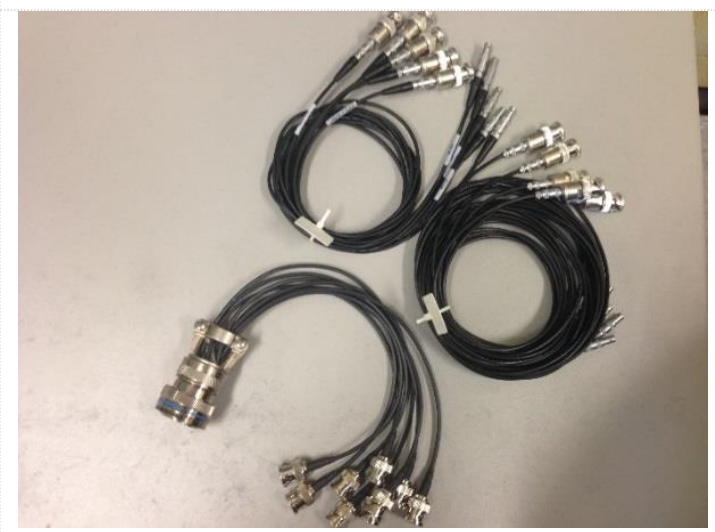

f.

Fig. 2.14 a. \& b. A 2-dimential multi-volume detector assembly, c. sealed and closed detector, d. \& e. Multi-pin plug (RG-170 cable soldered to the pin and inserted into the plug), f. custom made required signal cables: multi-pin plug - 9 BNCs, 9 signal cables LIMO - BNC 


\section{Double Layer THGEM Assembly}

For further investigation and optimization of the detector, two THGEM layers are used. This allows a better multiplication and results in a higher gain. In this case, everything is the same as before except having two layers of THGEM each biased using a separate HV divider as can be seen in Fig. 2.15 .

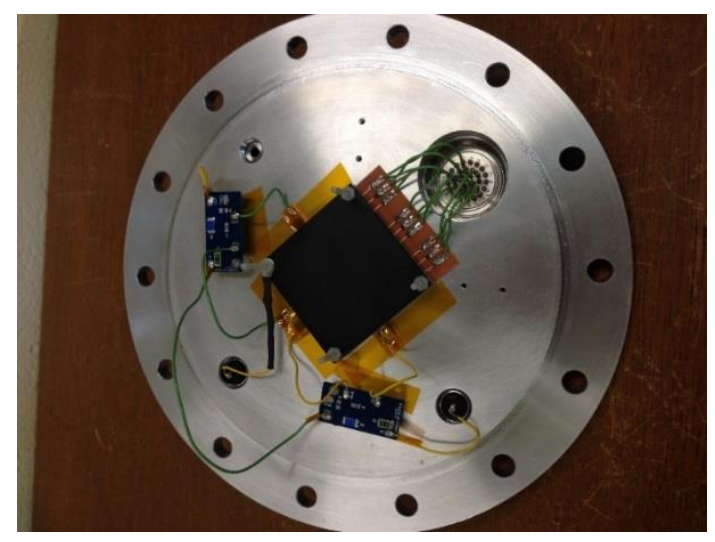

a.

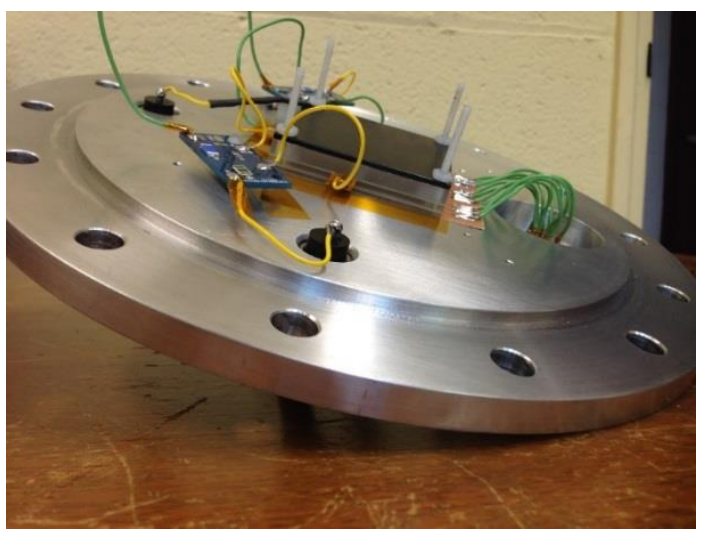

b.

Fig. 2.15 Double layer THGEM assembly a. Top view, b. Side view

The circuit diagram of detector wiring is shown in Fig. 2.16. In this case, the input HV is divided between top and bottom of two THGEMs by a factor using resistors $\mathrm{R}_{1}$ and $\mathrm{R}_{2}$ as below:

$$
\Delta V_{T H G E M 1 \& 2}=\frac{V 1_{\text {in }} \times 10 M \Omega}{(10 M \Omega+2 M \Omega) \times 2}
$$

The voltage difference between the $\mathrm{THGEM}_{1}$ bottom layer and $\mathrm{THGEM}_{2}$ top layer, known as $\Delta \mathrm{V}_{\text {Trans, }}$, and the voltage difference between the $\mathrm{THGEM}_{2}$ bottom layer and top layer of readout board, known as collection voltage, $\Delta \mathrm{V}_{\mathrm{CP}}$, are given below:

$$
\Delta V_{\text {Trans }}=\frac{V 1_{\text {in }} \times 2 M \Omega}{(10 M \Omega+2 M \Omega) \times 2}
$$




$$
\Delta V_{C P}=\frac{V 1_{\text {in }} \times 2 M \Omega}{(10 M \Omega+2 M \Omega) \times 2}
$$

Drift $\mathrm{HV}$ is the difference between the $\mathrm{V}_{\text {cathode }}$ and $\mathrm{V}_{\text {in }}$, which always is kept at least at 100 V.

$$
\Delta V_{\text {drift }}=V_{\text {cathode }}-V_{\text {in }} \geq 100 \mathrm{~V}
$$

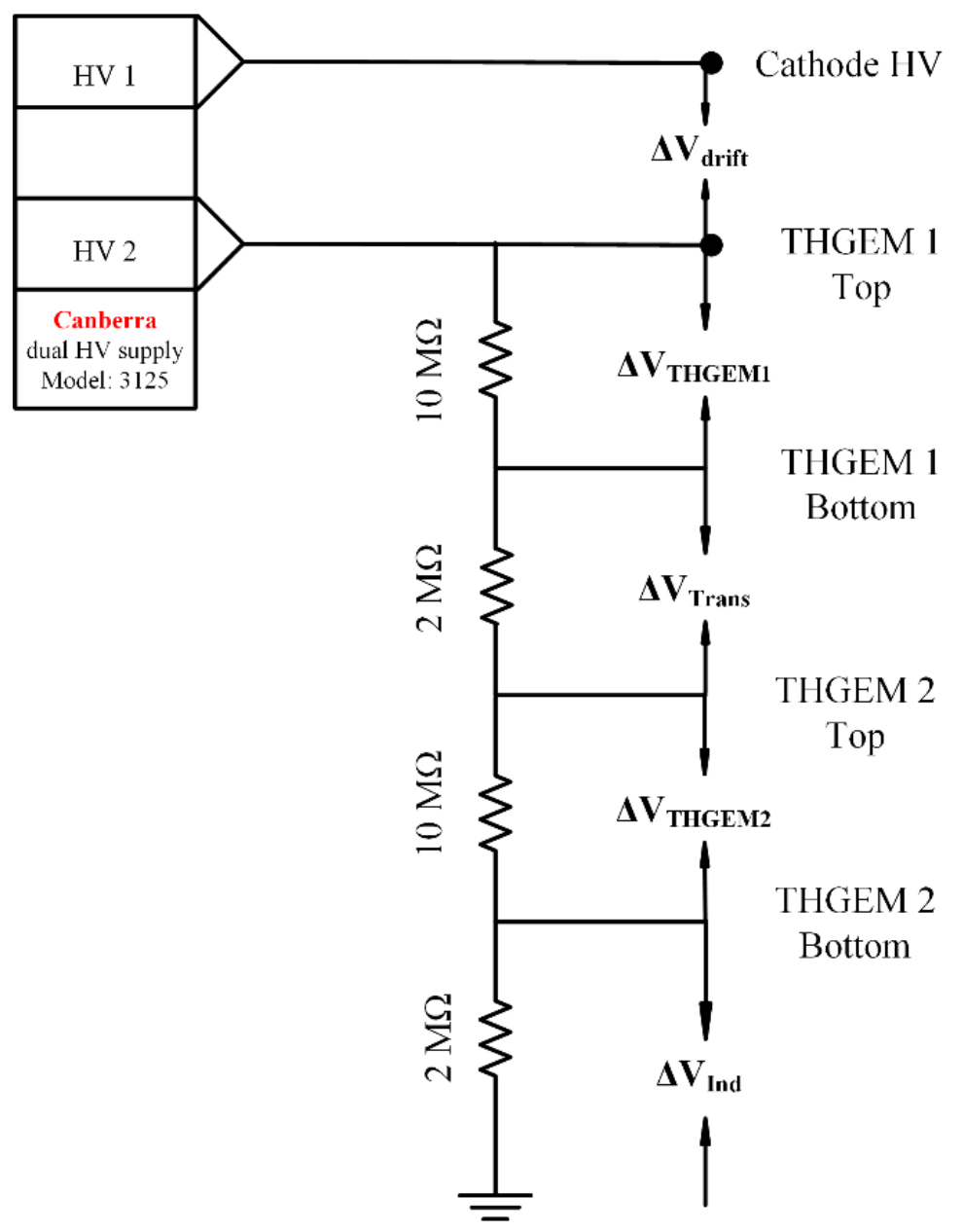

Fig. 2.16 High voltage connections circuit diagram for double THGEM layers 


\subsection{Smaller sensitive volumes}

To investigate how much smaller we can go for the gas sensitive volume and thus how much higher radiation field we would be able to measure with this detector, two different Rexolite layers were constructed with an array of $3 \times 3$, one with $2.5 \mathrm{~mm}$ and the other with $1 \mathrm{~mm}$ in diameter right cylinder sensitive volumes. As is apparent in Fig. 2.17, $2.5 \mathrm{~mm}$ size sensitive volume would cover 9 THGEM holes. However, $1 \mathrm{~mm}$ size sensitive volume only covers 1 THGEM hole. Thus it is worth testing both smaller size sensitive volume configurations. The only difference would be the pressure of filled gas which will be discussed in detail in the next section.

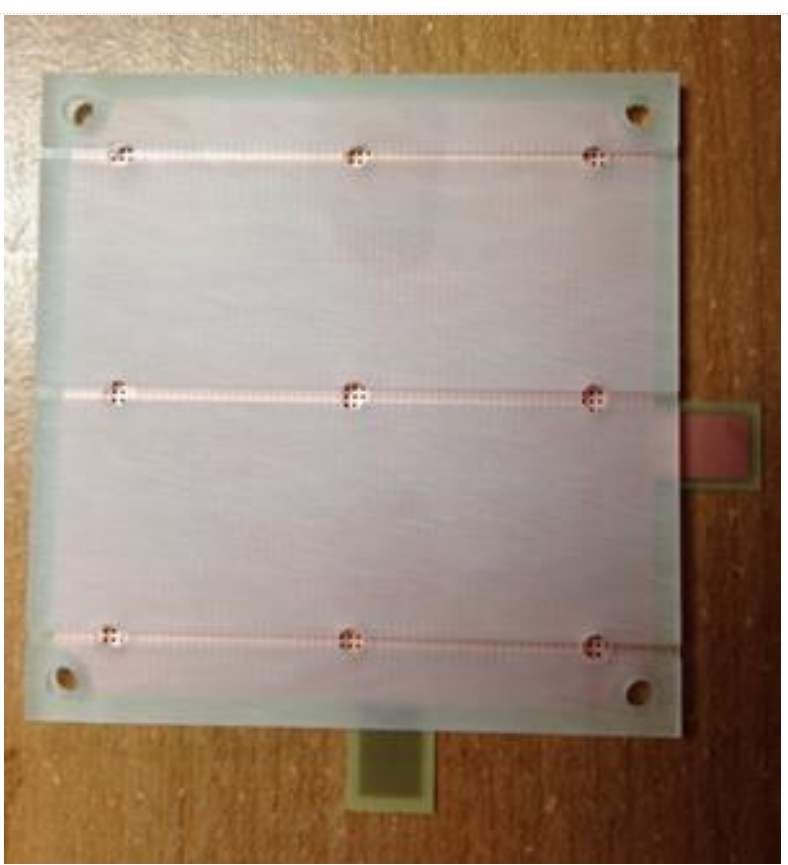

a.

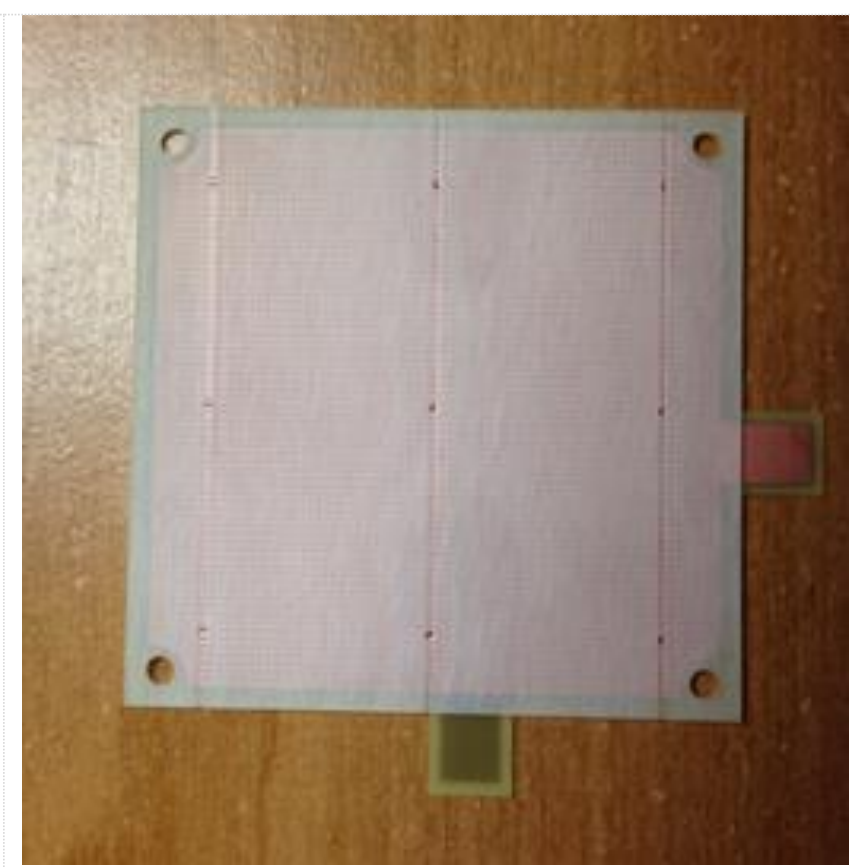

b.

Fig. 2.17 Array of $3 \times 3$ gas cavities with diameter of a. $2.5 \mathrm{~mm}$ and b. $1 \mathrm{~mm}$ 


\subsection{Vacuum test and gas filling}

Once detector wiring and assembly was performed, the chamber was sealed, closed and connected to an oil diffusion pump and pumped down to $10^{-4}$ torr for 2 days to evacuate trapped air and moisture as much as possible. Following the chamber pump down, the detector was filled with propane based tissue-equivalent gas. The pressure of the filling gas would be different depending on the intended size of the simulating unit density of soft tissue and the size of the sensitive volume. For a desired simulated diameter the gas pressure is determined by the cavity geometry and the composition of the gas. Assuming that the mass stopping powers are independent of the density, and the atomic composition of gas and walls are identical, the proper gas pressure, $\mathrm{P}_{\mathrm{g}}$, can be calculated as below:

$$
P_{g}=P_{0} \frac{\rho_{t}}{\rho_{g}} \frac{d_{t}}{d_{g}}
$$

$\mathrm{P}_{0}$ is the pressure of the gas in a standard condition, 750 torr, $\rho_{\mathrm{g}}$ is the gas density in standard

condition, $1.798 \mathrm{~kg} / \mathrm{m}^{3}, \rho_{\mathrm{t}}$ is the density of microscopic tissue volume, $1 \mathrm{~g} / \mathrm{cm}^{3} . d_{\mathrm{t}}$ and $d_{\mathrm{g}}$ denote desired simulated diameter and detector sensitive volume diameter respectively. Therefore in the cases of $5 \mathrm{~mm}, 2.5 \mathrm{~mm}$ and $1 \mathrm{~mm}$ sensitive volumes, the detector should be filled with gas at a pressure of 167 torr, 334 torr and 835 torr respectively to simulate a $2 \mu \mathrm{m}$ diameter of right cylinder unit density soft tissue.

The propane based tissue-equivalent gas is a certified gas mixture of $55 \%$ propane $\left(\mathrm{C}_{3} \mathrm{H}_{8}\right), 39.5 \%$ carbon dioxide $\left(\mathrm{CO}_{2}\right)$ and $5.5 \%$ nitrogen $\left(\mathrm{N}_{2}\right)$, purchased from MEGS Specialty Gases Inc., Canada. The vacuum pump and filling gas station which was used is shown in Fig. 2.18.

After a proper gas filling, the detector is ready to be exposed to a radiation field and be tested. 


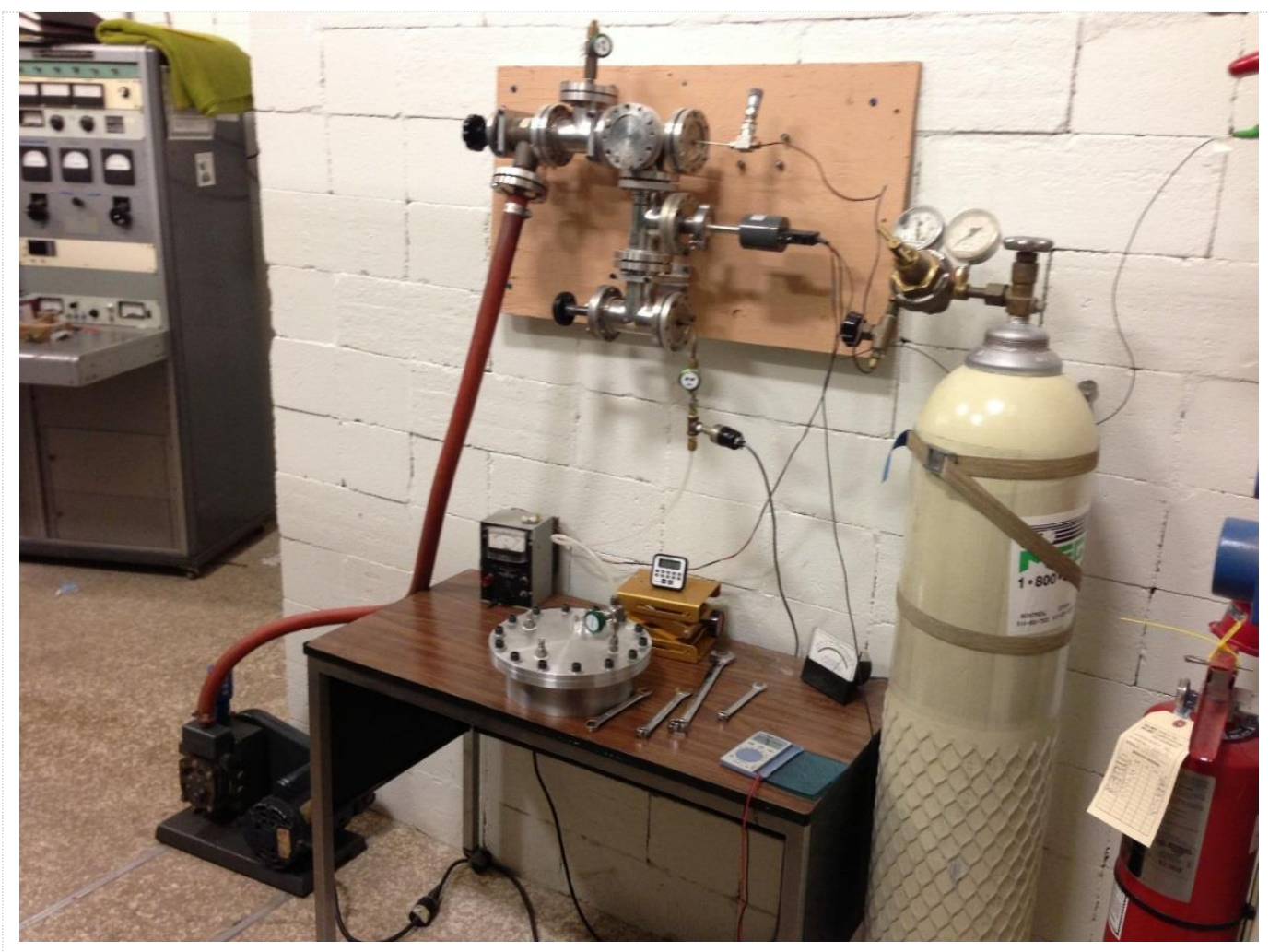

Fig. 2.18 Vacuum pump and filling gas station 


\section{Chapter 3}

\section{Development of McMaster Multi-Input Digital Signal Processing System (MMI-DSP)}

3.1 Design and Fabrication of McMaster Prototype Single-Input DSP (MSI-DSP)

3.2 Design Extension to the McMaster Multi-Input DSP (MMI-DSP)

3.3 Summary 


\section{Development of McMaster Multi-Input Digital Signal Processing System (MMI-DSP)}

The most important technical challenge that I faced in the phase of testing the $2 \mathrm{D}$ THGEM detector was how to process the signal outputs of 9 detectors simultaneously. The purchase of 9 commercial DSPs was not economically practical. Since there were no commercial products available with the required combination of sampling speed and multiinput with high throughput at very low cost, it was necessary to custom design a multiinput digital pulse processing system. The McMaster Multi-Input Digital Signal Processing System (MMI-DSP) was designed and fabricated by Kenrick Chin (department of Physics and Astronomy, McMaster University) to be used specifically with the 2D THGEM detector.

This chapter starts by explaining the architecture of the custom made prototype single input DSP (MSI-DSP) followed by a detailed presentation of the results of benchmark studies, of which the performance of MSI-DSP was compared to commercial analogue and digital signal processing systems. Ultimately, the extension of a single-input to multi-input DSP and its comparison with the commercial ORTEC DSPEC will be presented.

\subsection{McMaster Prototype Single-Input DSP (MSI-DSP)}

Commercial digital pulse height analyzers (PHA) use digital filters and trapezoidal pulse shaping to determine the pulse height. This takes time to compute in the DSP and may add to the dead time. However, MSI-DSP uses a simpler approach.

The prototype pulse processing system was developed using a microcontroller unit (MCU) (model STM32F407, STMicroelectronics) interfaced with an ADS807 12-bit sampling ADC (Analogue to Digital Converter) with a sampling rate of $42 \mathrm{Msps}$, which offers a considerably inexpensive solution for processing multiple detectors (see Fig. 3.1 and $3.2 \mathrm{a}$ ). First, the preamplifier pulse shape is sampled and averaged over a large number of pulses to create a normalized pulse shape. This is stored in the MCU as a reference pulse. The incoming signal is continuously digitized by the ADC and recorded in an 8192-word memory. Hence the data buffer contains about $195 \mu$ s worth of data. The sampling process 
is continuous and the data stored in a circular buffer, i.e. data are continuously being analyzed while the data buffer is being filled. Hence there is no loss of data. In other words, the software system does not wait for a full buffer but begins analysis once there are sufficient data in the buffer. The ADC digitizes continuously without interruption. The analysis algorithm is optimized such that analysis keeps up with data acquired. Assuming that the MCU can process 8192 data points in less than $195 \mu$ s, the theoretical dead time is zero.

The analysis consists of searching for a significant rise in signal voltage. A positive edge detected while the signal has not returned to baseline is treated as pulse pile-up (a secondary pulse). The time interval between the primary pulse and the secondary pulse is determined by detecting the peak occurrences of the two pulses. The tail of the primary pulse is estimated from the reference pulse knowing the amplitude of the primary pulse and the time of arrival of the secondary pulse. The amplitude of the secondary pulse is hence forth corrected. A similar correction is applied for any subsequent pile-up events and thus the system is capable of processing any number of pulse pile-ups in succession.

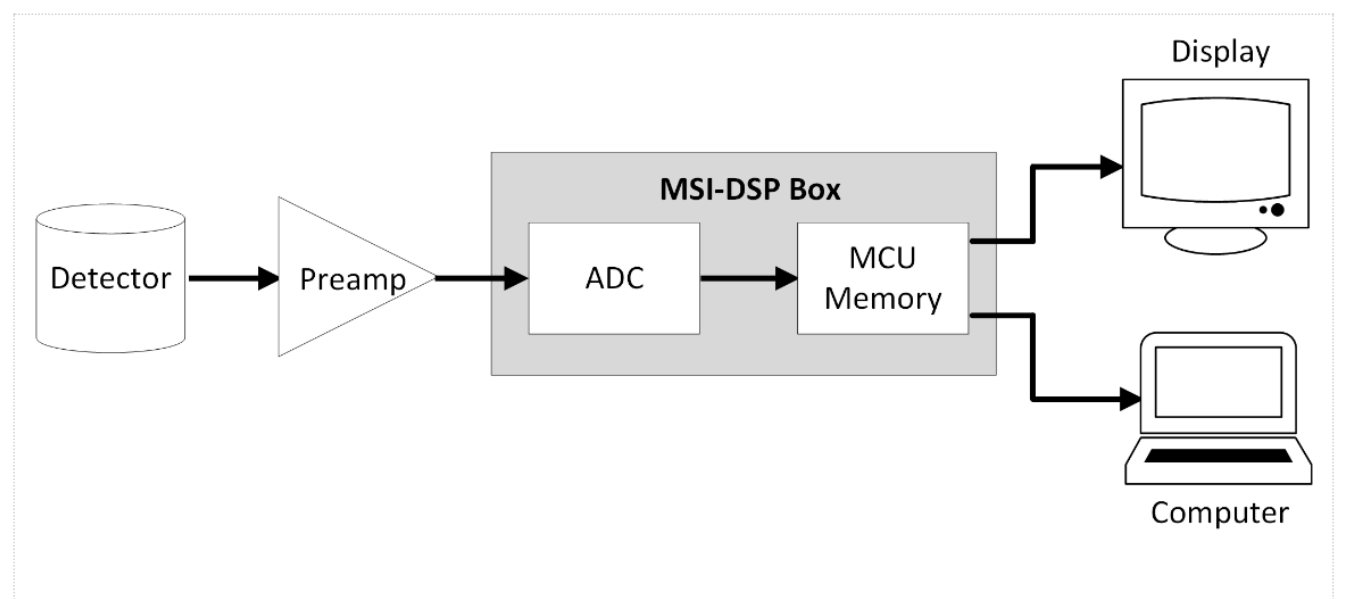

Fig. 3.1 Block diagram of the pulse processing system and prototype MSI-DSP, showing the major functional sections 
The MCU was controlled via MATLAB software and connected to a display through VGA port for real-time visualization. There is a USB-2 high-speed connectivity, which was used to connect to the Laptop for data transfer. The MSI-DSP board was enclosed in an Aluminum box, shown in Fig. 3.2 b.

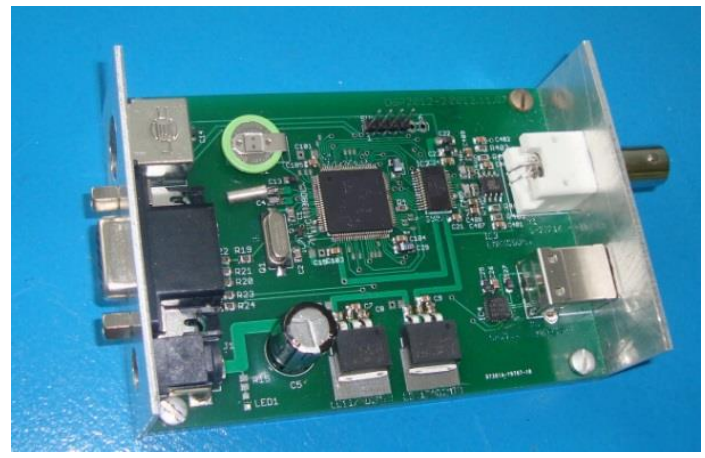

a.

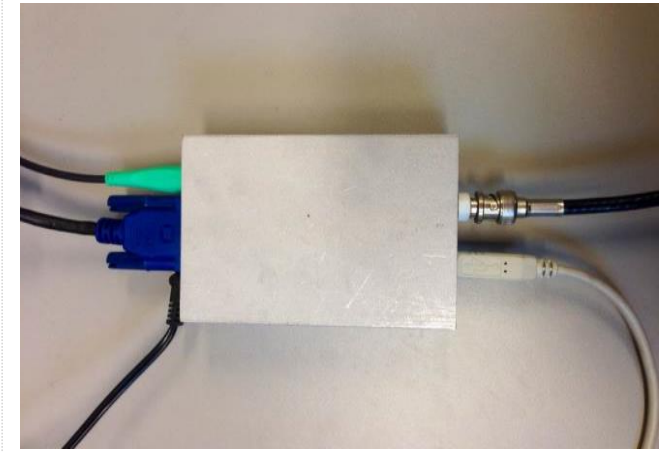

b.

Fig. 3.2 a. MSI-DSP prototype printed circuit board, b. MSI-DSP prototype box

\section{MSI-DSP vs. Commercial Digital and Analogue Signal Processing Systems (Gamma-ray calibration source)}

The functionality of the prototype system was extensively investigated using a $\mathrm{NaI}(\mathrm{Tl})$ detector and compared to a traditional ORTEC analogue system (Spectrum Master Model: 919) and ORTEC commercial digital system (Model: DSPEC).

Several benchmark tests were performed using $\mathrm{NaI}(\mathrm{Tl})$ detector and gamma-ray calibration sources, to verify the performances of our custom made DSP. Fig. 3.3 shows the experimental setup. $950 \mathrm{~V}$ high voltage was applied to a NaI detector (Harshaw chemical Co. Crystal \& Electronics products, model: 20MB16/5A, serial: MR 359). The detector was connected to a preamplifier (ORTEC, model: 113). The output of the preamplifier was connected to the three signal processing systems individually.

The sources used were ${ }^{137} \mathrm{Cs}$ and ${ }^{60} \mathrm{Co}$. The interaction rate through the detector was adjusted by using 20 different ${ }^{137} \mathrm{Cs}$ sources of varying strength and also by adjusting the distance between the source and the detector to achieve the desirable count rates, which were $3,41,76$ and $142 \mathrm{kcps}$. 


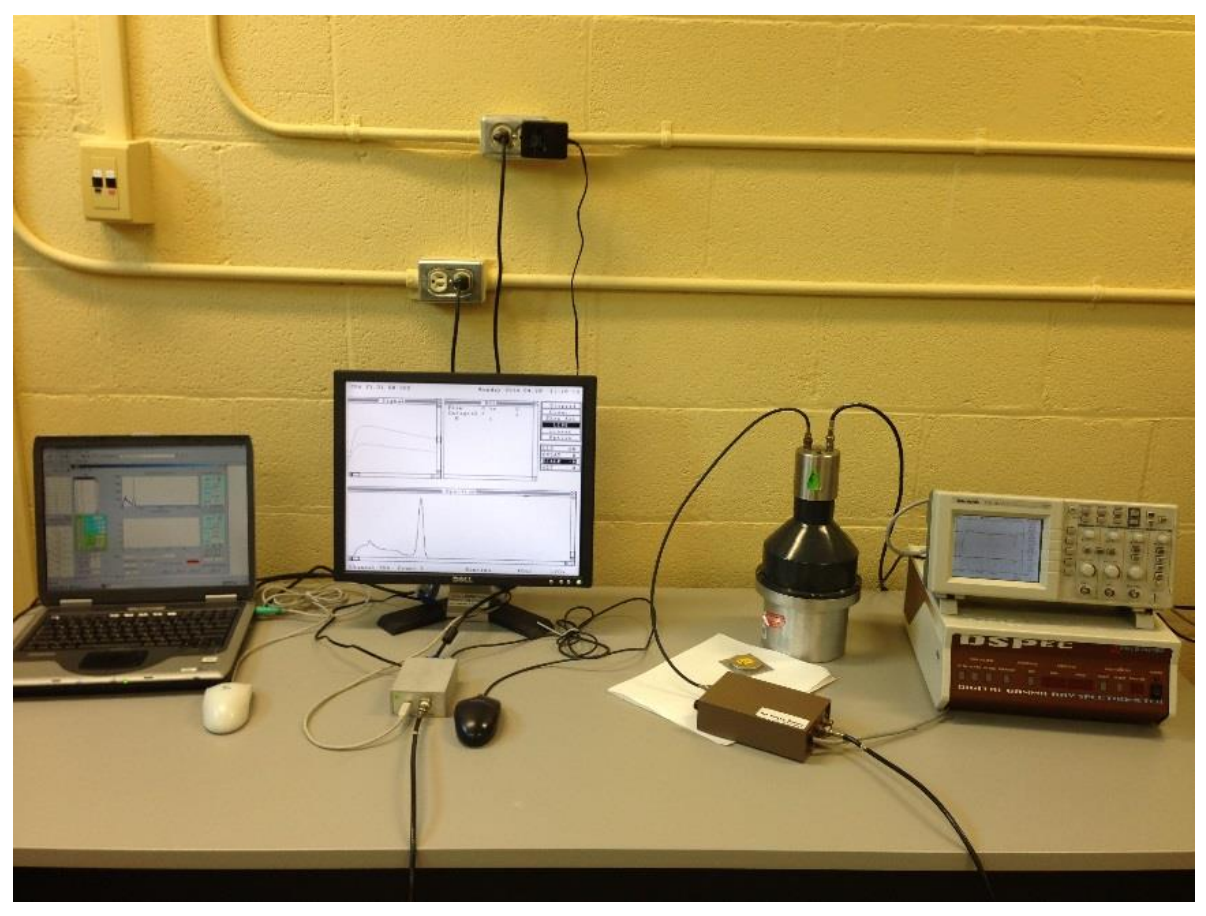

Fig. 3.3 Experimental setup using NaI detector, MSI-DSP, ORTEC DSPEC and Analogue signal processing systems

Before the results are presented, it is worth briefly explaining the controller interface of MSI-DSP and how to use it efficiently. First, through MATLAB, the program called DSP 9 should be run. The controller interface will pop up as shown in Fig. 3.4. This is the window from which everything can be controlled.

Before an experiment begins, two calibration steps must be performed:

1) Calibrate the baseline: The first measurement determines the baseline reference voltage with no signal applied to the input of the ADC. Since the input signal is AC coupled to the internal amplifier this calibration measures the "zero-signal" input level of the ADC. For the 12-bit ADC, this reference level is nominally around a digital value of 2048. The ADC is capable of measuring bipolar signals, from $-1 \mathrm{~V}$ to $+1 \mathrm{~V}$. For uni-polar signals, this reduces the effective number of ADC bits to eleven instead of twelve.

2) Calibrate the reference pulse: The second measurement requires input signals at relatively low count rates. The purpose of this measurement is to acquire a representative 
shape of all detector pulses. In the pulse-height analysis, the assumption is made that all pulses have the same characteristic shape independent of voltage. From this reference pulse shape, an estimate of the decay time is obtained. This is used in correcting for piled-up pulses.

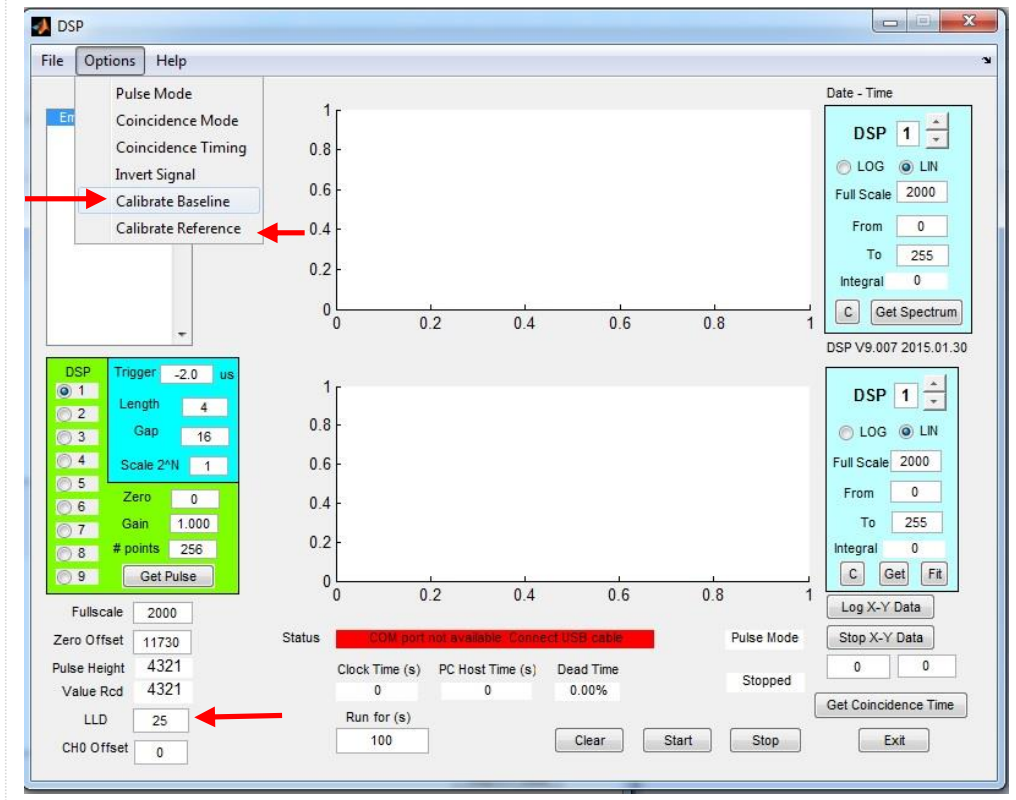

Fig. 3.4 MSI-DSP controller interface

In the prototype MSI-DSP, there is an option for the display connectivity, which allows the user to control the settings and see the spectrum in real time (see Fig. 3.5). As shown, there are 3 windows, one for displaying the preamp signal (works as an oscilloscope), one to display the real time spectrum and one for the region of interest (ROI) information. The screen can be deactivated and all the settings are done through the MATLAB DSP 9 interface.

After preliminary tests, it was found that having the display screen active, caused a $22 \%$ decrease in the total count rate compared to the case when the display screen was deactivated. Hence, all the final measurements for prototype MSI-DSP were carried out using the MATLAB DSP 9 interface with the display screen deactivated. 


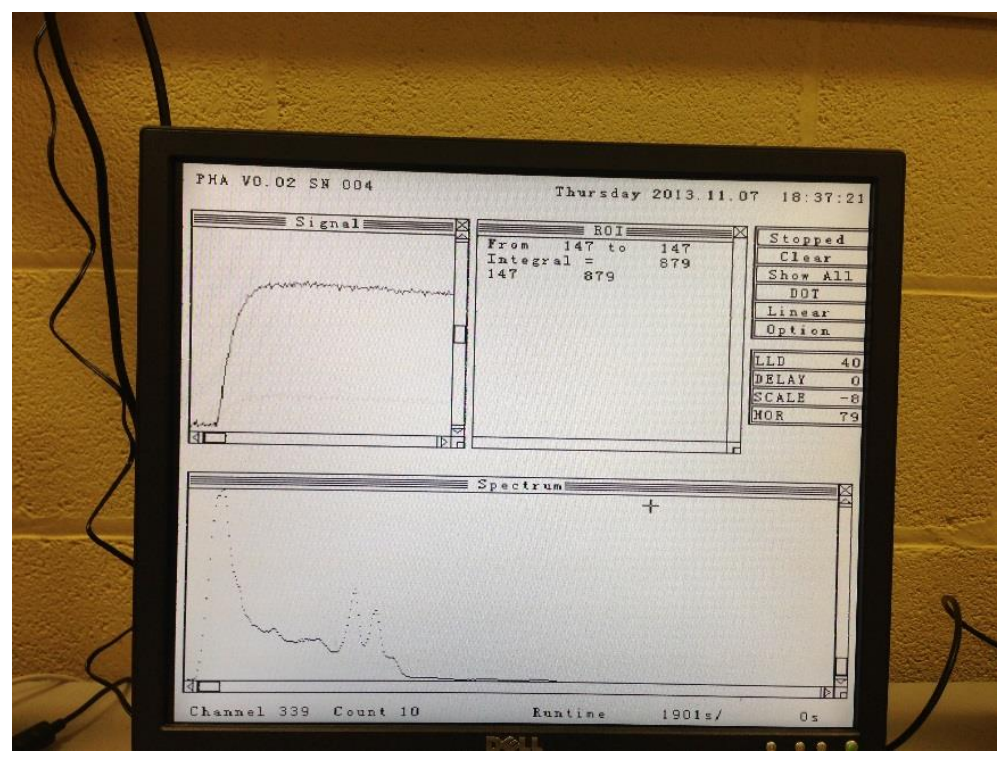

Fig. 3.5 MSI-DSP display screen

The MSI-DSP system was designed to detect and count all pulses including pulses riding on the tail of a prior pulse, what is known as "pulse pile-up". In other words, all pulses are analyzed for possible pulse pile-up. The search for a pulse begins with finding a positive first derivative that exceeds a threshold value. A refined search determines the position of the leading edge and the value of the baseline immediately prior to the leading edge. Next, the pulse peak and position of the peak is located. The tentative pulse-height is the difference between the peak value and the leading baseline value.

The second part of the analysis corrects for any possibility of the pile-up. Any difference of the baseline from the "zero-level" baseline is considered a candidate for pile-up correction. Knowing the baseline value and the time to peak, an estimate of the correction is calculated using the previously stored reference pulse shape. This correction is applied to the previously determined estimate of the pulse-height. Because the system software is capable of detecting and separating pile-ups from non-pile-up pulses, it was possible to acquire the data in separate spectra during the development of the system. By doing this, the validity and accuracy of the algorithms and analyses were ascertained. 
In MSI-DSP, the concept of "dead-time" is no longer relevant. Instead of "dead-time" the concept of "missed pulses" is more appropriate. Any pile-up that occurs within the leading edge of a prior pulse would be missed and not counted. A pulse appearing after the peak of a prior pulse is detected, corrected and counted. Thus the percentage of missed pulses is a direct function of the rise time of the pulses in coincidence. However, for convenience, we will keep the name "dead time" when presenting results.

Setting a proper gain on the DSP is another important factor that has to be considered. In the existing version of prototype MSI-DSP, there is no option of changing gain through the software. The gain change must be done on the printed circuit board by changing two resistors $\mathrm{R}_{1}$ and $\mathrm{R}_{3}$, shown in Fig. 3.6 to achieve a desirable gain using eq. 3-1:

$$
G=\frac{R_{3}}{R_{1}}
$$

$\mathrm{R}_{2}$ and $\mathrm{R}_{4}$ are also changed to match $\mathrm{R}_{1}$ and $\mathrm{R}_{3}$, respectively.

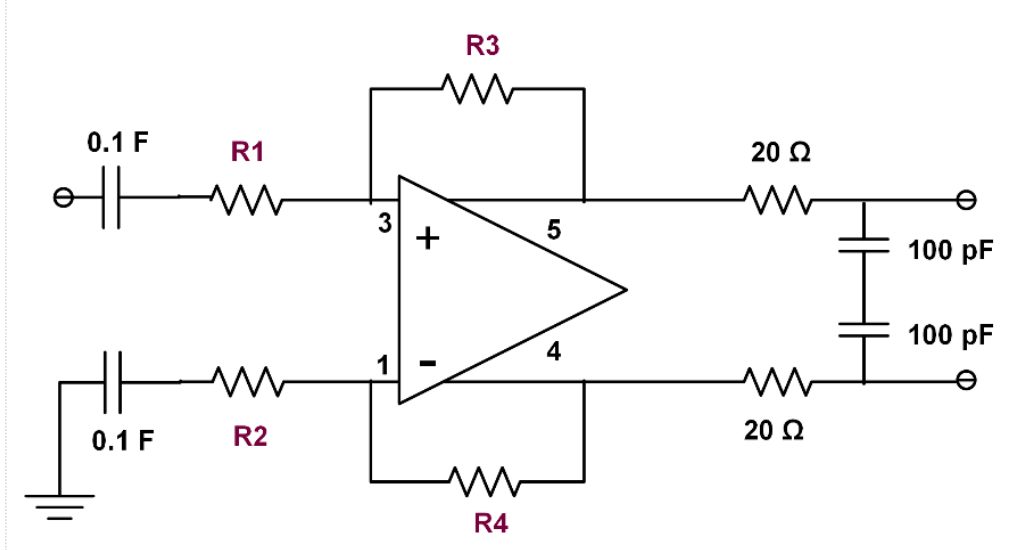

Fig. 3.6 MSI-DSP gain setting block diagram

Thus to have a gain of 10 on MSI-DSP, $R_{1}$ and $R_{3}$ were chosen to be $100 \Omega$ and $1 \mathrm{k} \Omega$ respectively. Depending on what gain is required, $\mathrm{R}_{1}$ and $\mathrm{R}_{3}$ are chosen accordingly. 
In the next subsections, comprehensive results for rate performances, dead time and resolution will be discussed and compared to commercial ORTEC analogue and digital pulse processing systems thoroughly.

\section{Test Results}

Figs. 3.7 to 3.10 show the gamma-ray spectra collected for the 4 different count rates, mentioned above (3.1 to $78 \mathrm{kcps}$ ), using 3 type of signal processing systems: MSI-DSP, ORTEC DSPEC and ORTEC analogue system. The gain settings on DSPEC and analogue amplifier were adjusted to bring the energy spectrum in alignment with that of MSI-DSP. Comparing MSI-DSP gain setting to that of DSPEC, it was found that the gain setting of 10 on MSI-DSP is almost equal to a gain setting of 8.4 on DSPEC.

The real-time performance of the pulse pile-up recovery algorithm in MSI-DSP is presented in table 3-1 (in terms of \% resolution and \% dead-time) with increasing input count rate. In Figs. 3.11 to 3.13 , these values are compared to the ones calculated for the DSPEC and analogue system.

It was found that for a relatively high count rate of $61 \mathrm{kcps}$, the dead time was $28 \%$ less than DSPEC and $144 \%$ less than analogue system. When the count rate increased to $78 \mathrm{kcps}$ the dead time increased by $11 \%$ compared to DSPEC. However, it was still $44 \%$ lower than the dead time for the analogue system.

Consequently, the area under the ${ }^{137} \mathrm{Cs}$ peak was found to be $10 \%$ higher than DSPEC and $35 \%$ higher than an analogue system for count rate of $61 \mathrm{kcps}$. For the count rate of $78 \mathrm{kcps}$, the area under the ${ }^{137} \mathrm{Cs}$ peak was $12.6 \%$ less than DSPEC and $26 \%$ higher than an anague system.

Across a 47-fold increase in input count rate, the detector resolution for the $662 \mathrm{keV}$ peak from ${ }^{137} \mathrm{Cs}$ degrades by less than $32 \%$ from a minimum of $8.5 \%$ to a maximum $12.6 \%$ for MSIDSP and degrades by $40 \%$ from a minimum of $8.6 \%$ to a maximum of $14.3 \%$ for DSPEC.

The results comparing MSI-DSP and DSPEC for NaI detector using an accelerator neutron source will be discussed in chapter 6 , where all the 2D THGEM detector results will be presented.

Therefore, for the purpose of this thesis (development of a 2D THGEM detector), in which the count rates would not be more than approximately $1 \mathrm{kcps}$, the performance of MSI-DSP 
compared to commercial digital and analogue systems is superior and the results are satisfactory. In the next section, the extension of the design to multi-input DSP will be described.

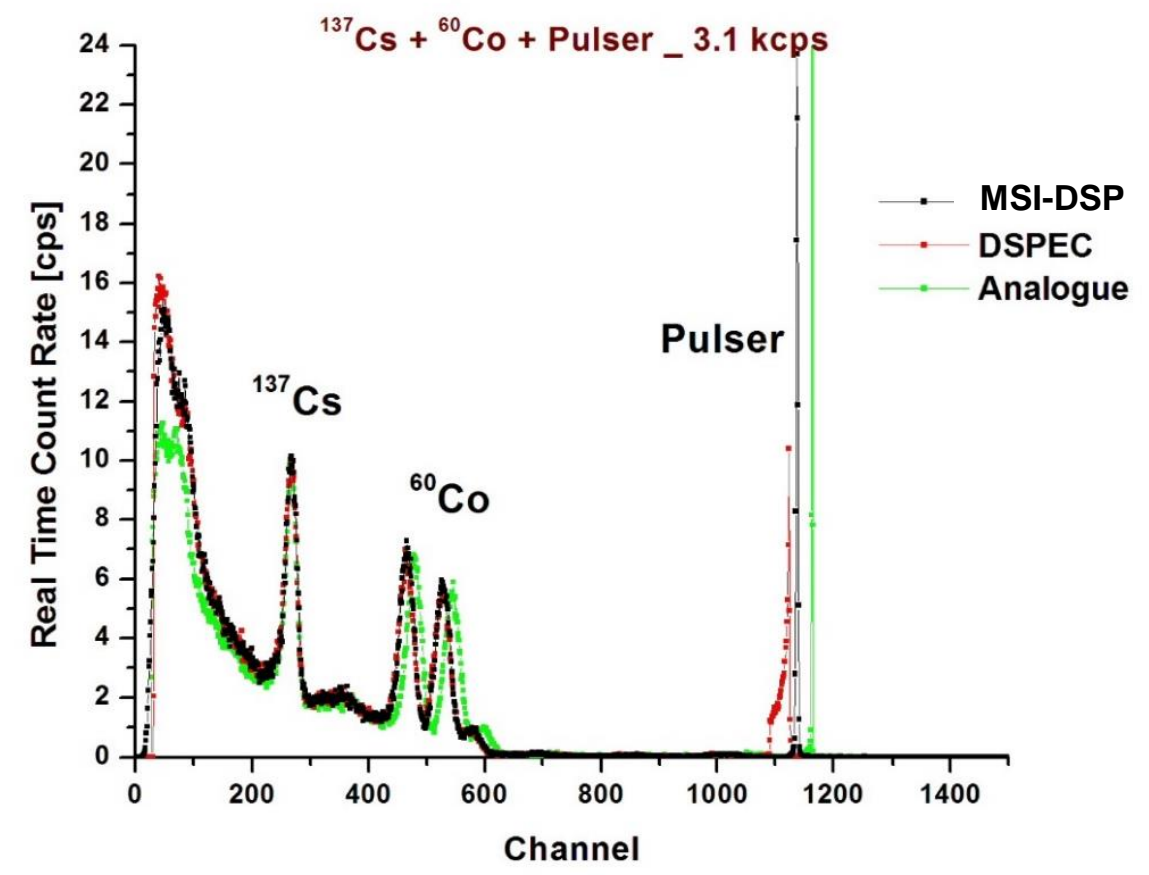

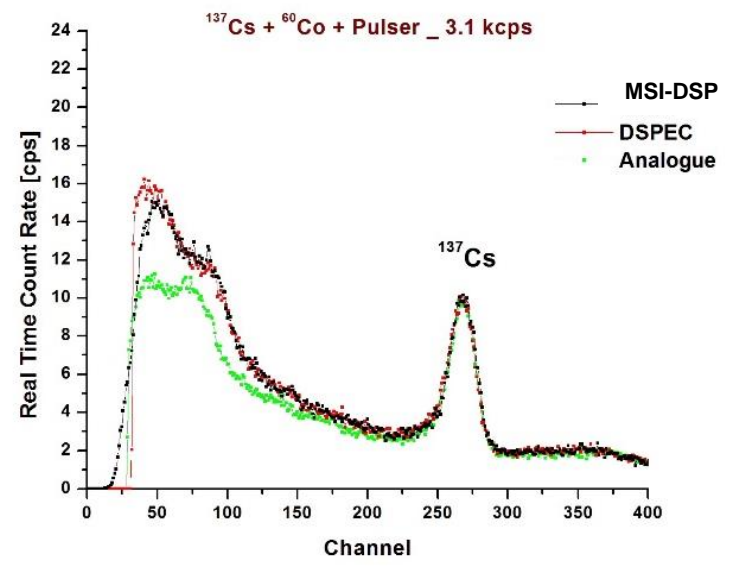

b.

a.

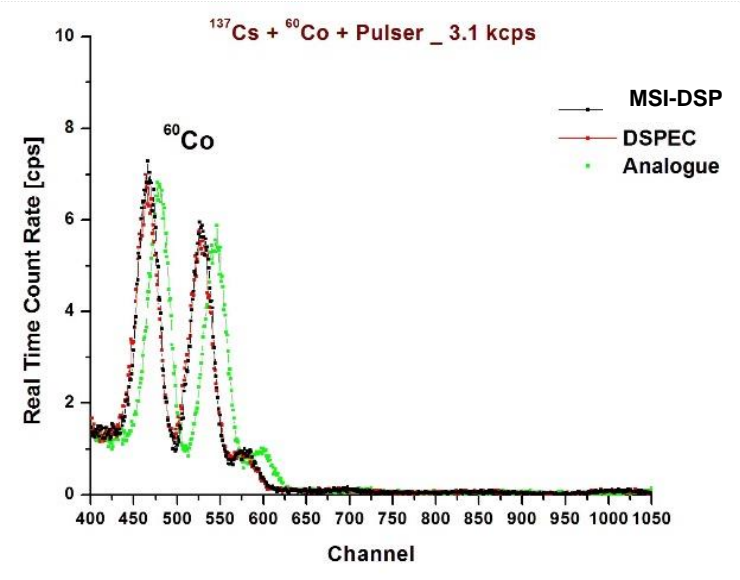

c.

Fig. 3.7 a. Gamma-ray spectrum for low count rate: $3.1 \mathrm{kcps}$, b. Zoom in of lower channel numbers to show ${ }^{137} \mathrm{Cs}$ peak, $\mathbf{c}$. Zoom in of higher channel numbers to show ${ }^{60} \mathrm{Co}$ peak 


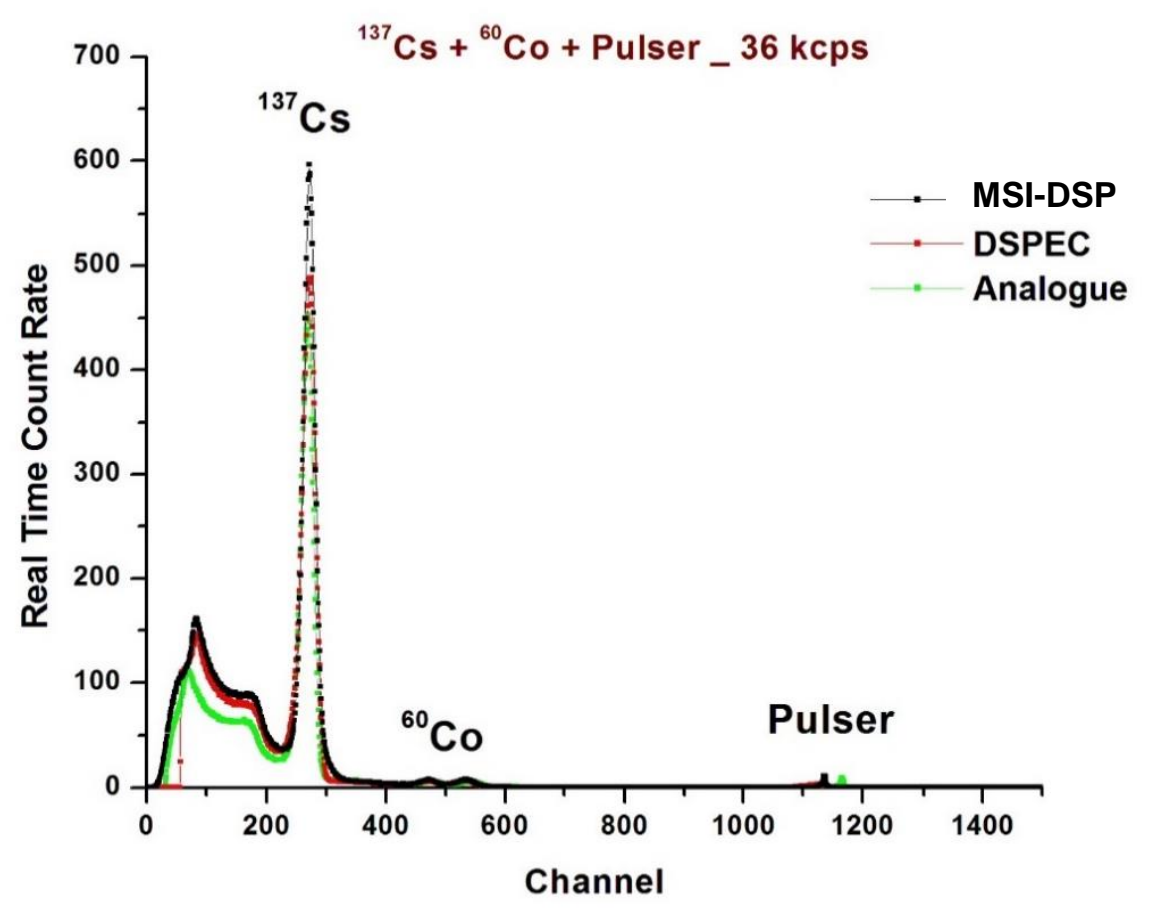

a.

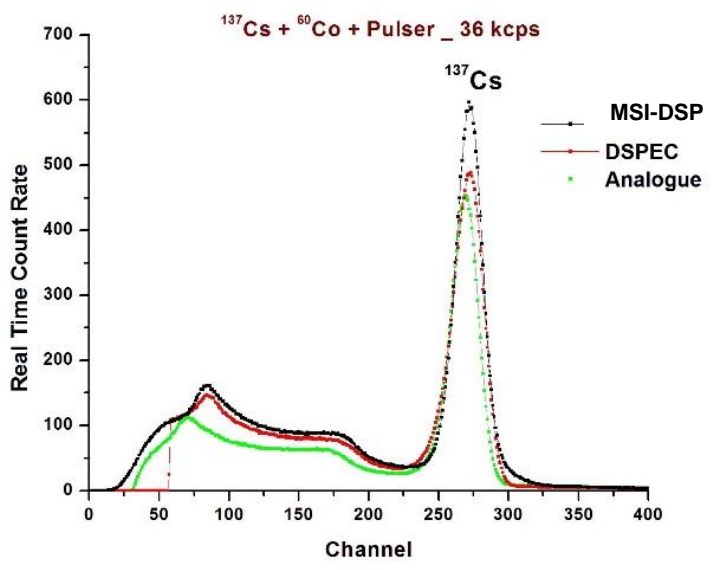

b.

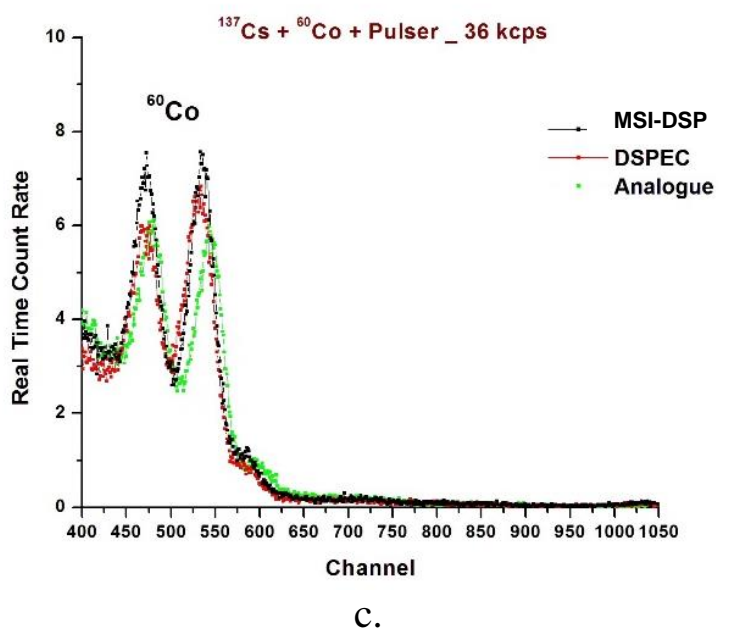

Fig. 3.8 a. Gamma-ray spectrum for count rate: $36 \mathrm{kcps}$, b. Zoom in of lower channel numbers to show ${ }^{137} \mathrm{Cs}$ peak, c. Zoom in of higher channel numbers to show ${ }^{60} \mathrm{Co}$ peak 


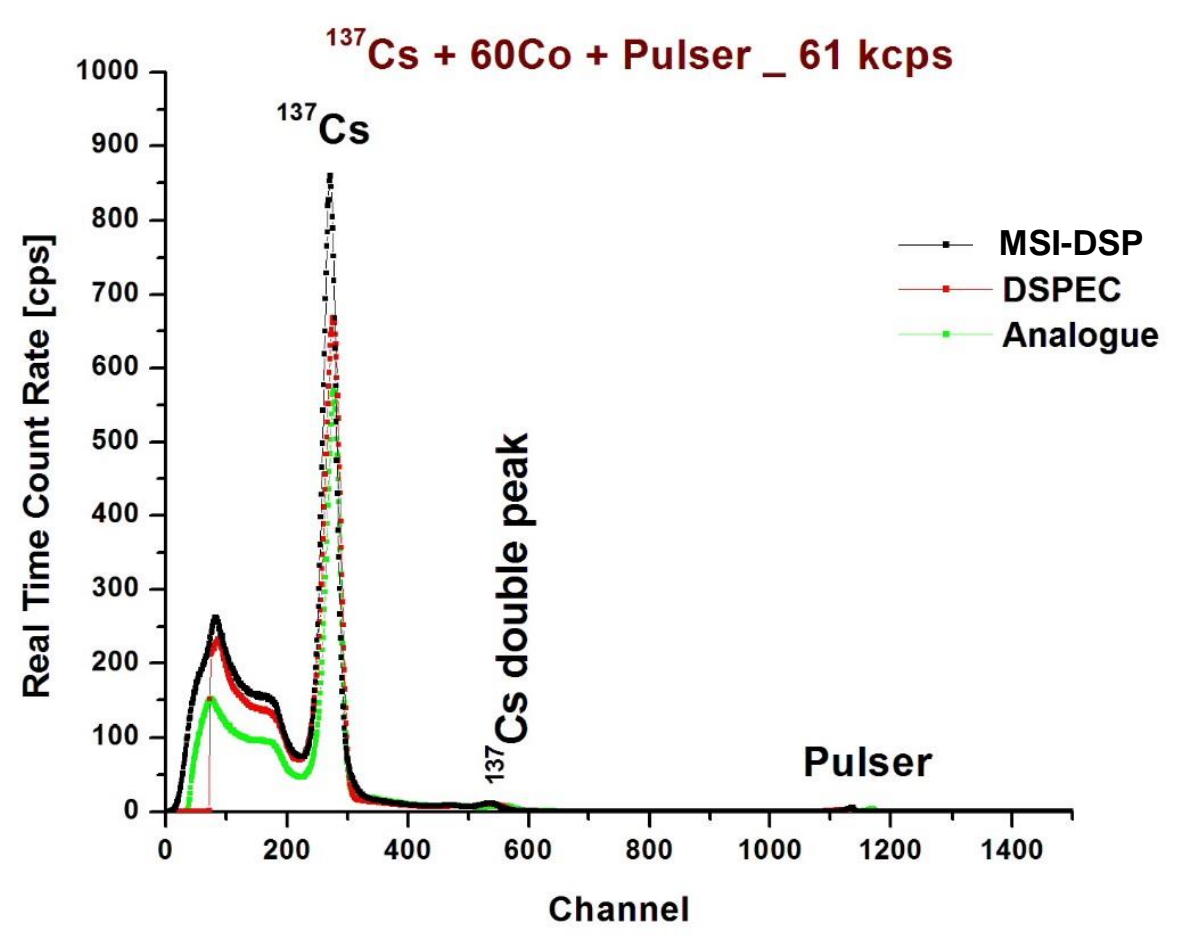

a.

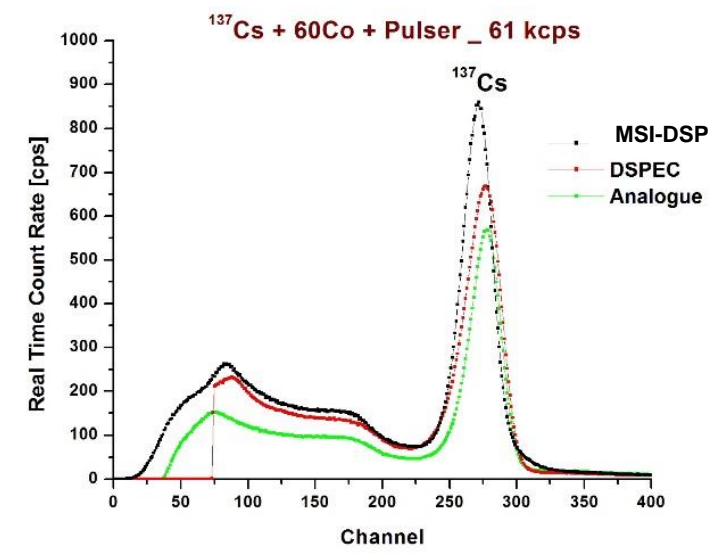

b.

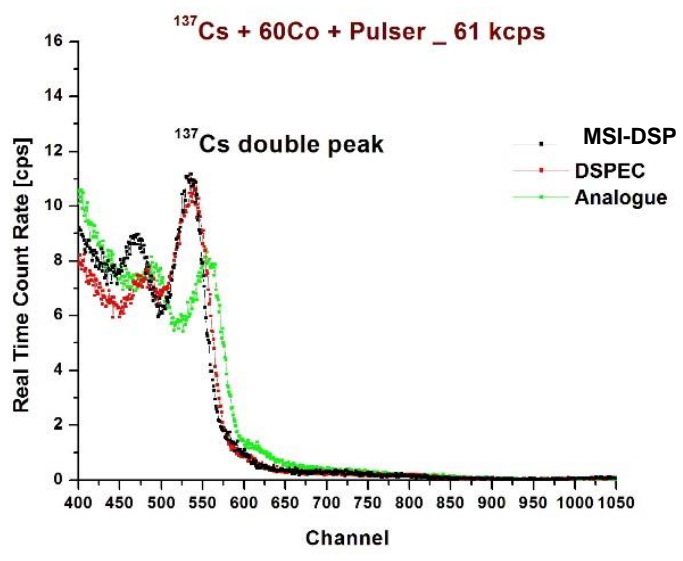

c.

Fig. 3.9 a. Gamma-ray spectrum for count rate: $61 \mathrm{kcps}$, b. Zoom in of lower channel numbers to show ${ }^{137} \mathrm{Cs}$ peak, c. Zoom in of higher channel numbers to show ${ }^{60} \mathrm{Co}$ peak 


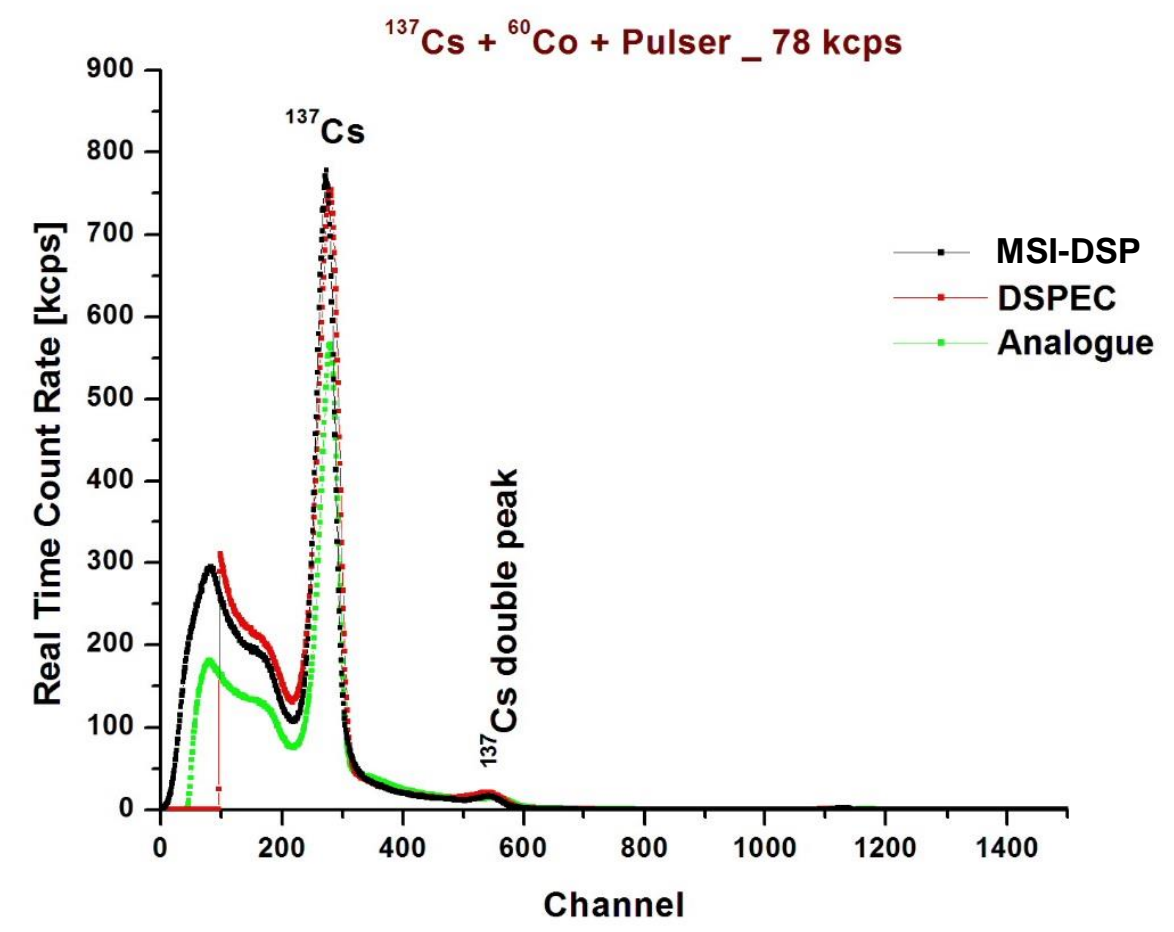

a.

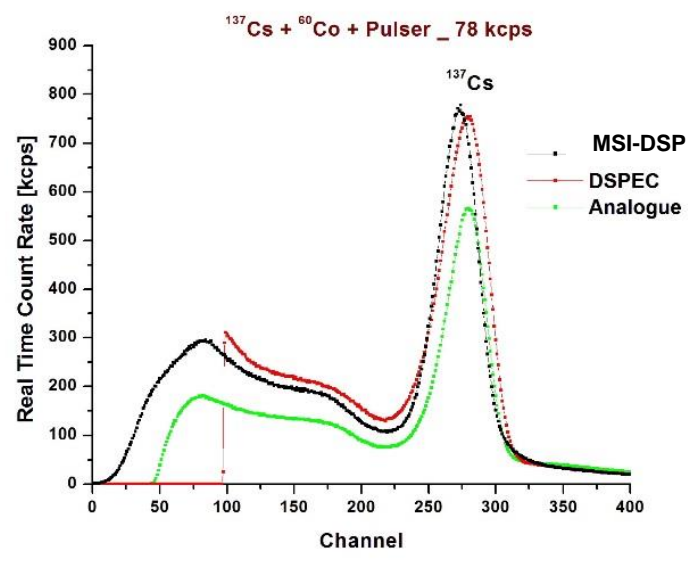

b.

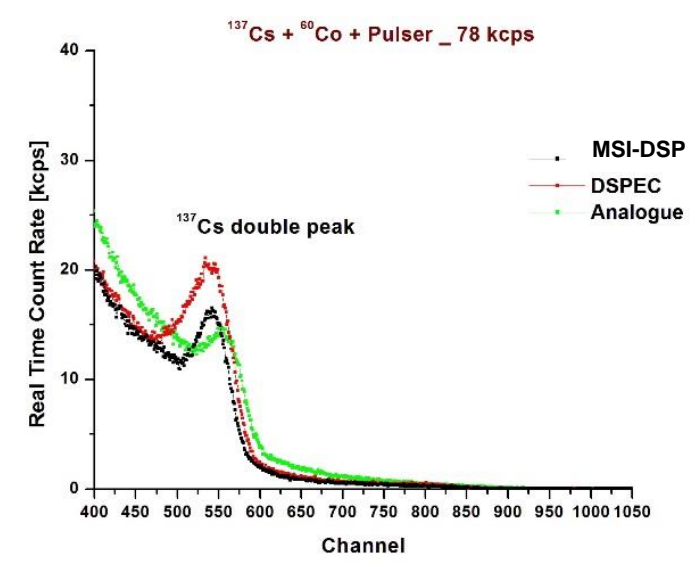

c.

Fig. 3.10 a. Gamma-ray spectrum for count rate: $78 \mathrm{kcps}$, b. Zoom in of lower channel numbers to show ${ }^{137} \mathrm{Cs}$ peak, c. Zoom in of higher channel numbers to show ${ }^{60} \mathrm{Co}$ peak 
Table 3-1 The real-time performance of the pulse pile-up recovery algorithm in MSI-DSP, in terms of rate performances, dead time and resolution for the $662 \mathrm{keV}$ energy peak from ${ }^{137} \mathrm{Cs}$

\begin{tabular}{cccc}
\hline Count Rate & \multicolumn{3}{c}{ Prototype MSI-DSP } \\
\cline { 2 - 4 }$[\mathrm{kcps}]$ & Area under ${ }^{137}$ Cs peak & Dead Time \% & Resolution \% \\
\hline $\mathbf{3 . 1}$ & 0.2 & 1.6 & 8.5 \\
$\mathbf{3 6}$ & 13 & 13 & 8.5 \\
$\mathbf{6 1}$ & 23 & 18 & 10 \\
$\mathbf{7 8}$ & 25.4 & 45 & 12.6 \\
\hline
\end{tabular}

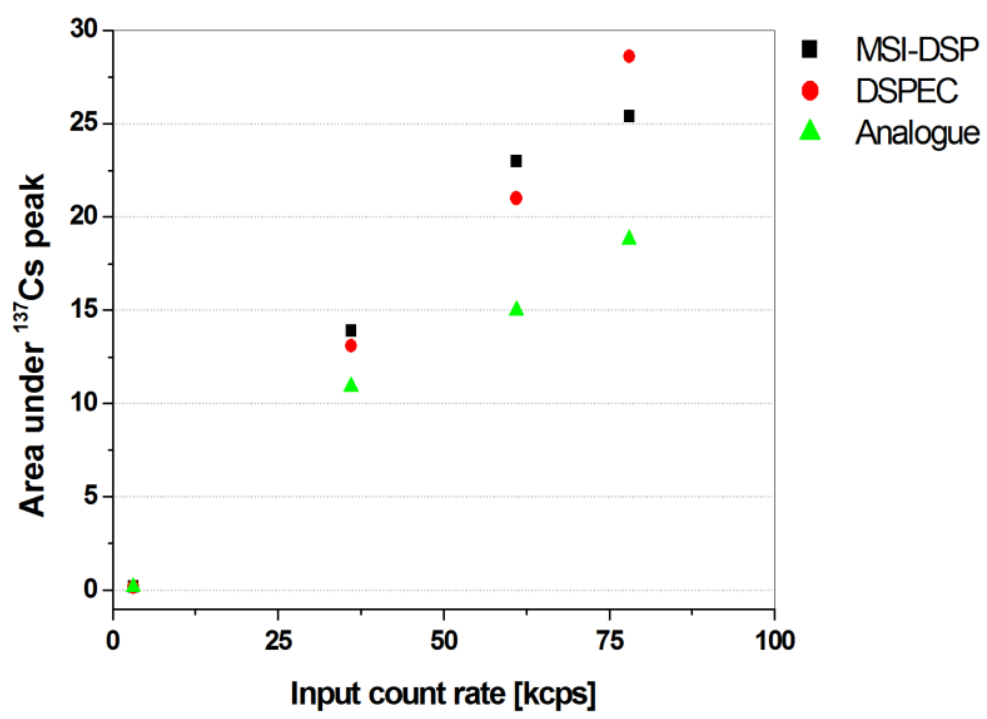

Fig. 3.11 Rate performances of MSI-DSP vs. DSPEC and Analogue systems 


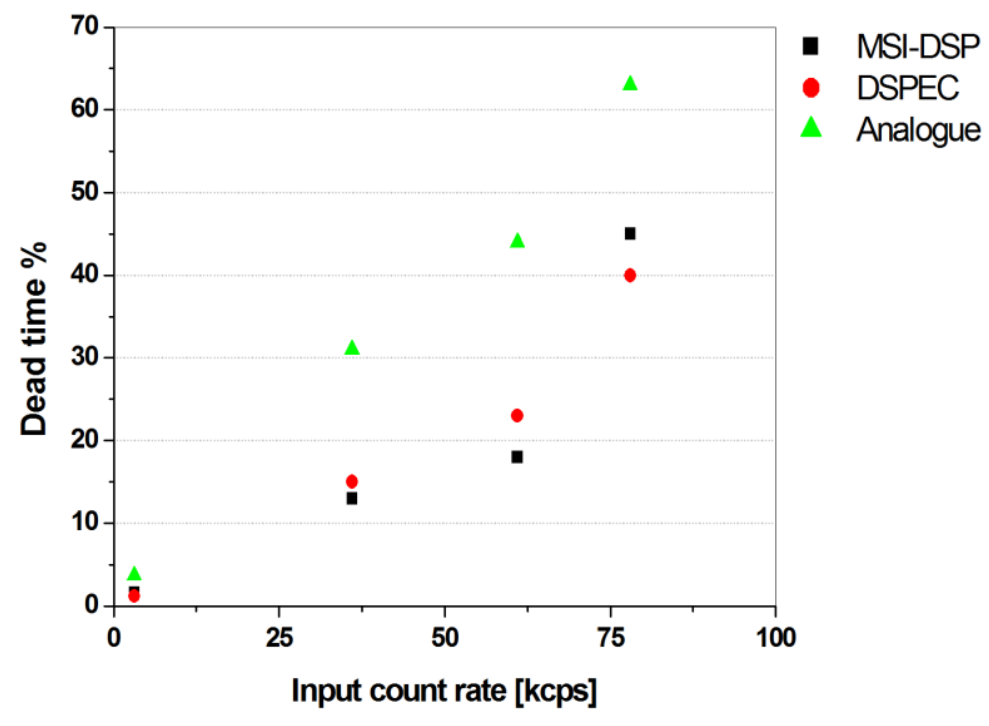

Fig. 3.12 Dead time of MSI-DSP vs. DSPEC and Analogue systems for different count rates

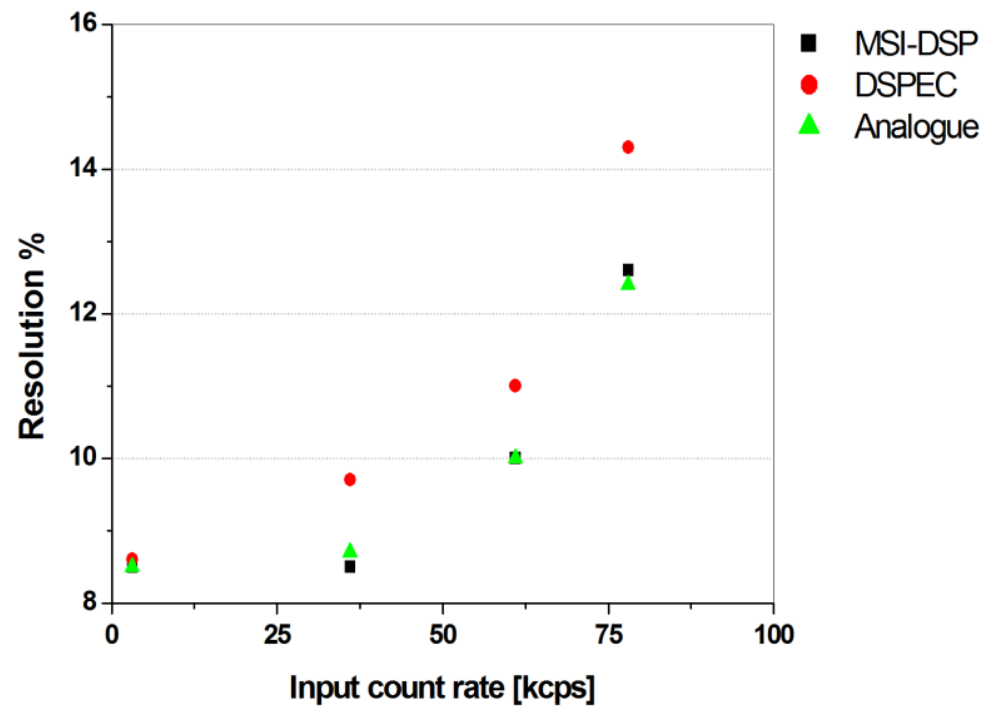

Fig. 3.13 Resolution of MSI-DSP vs. DSPEC and Analogue systems for different count rates 


\subsection{Design Extension to the McMaster Multi-Input DSP (MMI-DSP)}

As the results of the benchmark test, obtained above, demonstrated the satisfactory performance of the single input DSP, the design was extended to multi-input DSP. The prototype single-input design was slightly changed and miniaturized in the way that each board can accommodate 5 DSP circuits simultaneously, as shown in Fig. 3.14 a. In this case, on each board, there is only one power connection and one USB connection through which all 5 DSPs are biased and connected to the acquisition system. As the display has no application in multi-input DSP, the VGA port was eliminated in the extended design. This design has the capability to extend to as many DSP as are needed just by adding boards, consisting of 5 inputs. As illustrated in Fig. 3.14 b and $c$, for convenience, it was decided to assemble the DSP boards on a 3 unit panel of a 19" rack. The design drawing is shown in Fig. 3.15. To this end, grooves of 1" apart were machined into two plastic rods, bolted to the panel, so that the boards can slide in. Then a thin metal rod was passed through all the boards and was fixed at the both ends using threaded nuts. Two holes were machined on the panel for power connectors. Likewise, 5 holes of 3/8" size were machined on the panel in front of each board, for BNC connectors. Ultimately, each board was wired and each DSP was connected to one BNC and was labelled accordingly.

In order to avoid any further ambiguity, the first board performance was investigated and compared to the single-input DSP prior to assembling the second board. The experimental setup is illustrated in Fig. 3.16. Using a NaI detector the gamma spectrum of a ${ }^{137}$ Cs source was collected from each 5 channels of MMI-DSP(5) individually. As can be seen in Fig. 3.17, the spectrum of each DSP channel are aligned and consistent.

Fig. $3.18 a$ and $b$ show the gamma spectra collected with MSI-DSP, MMI-DSP(5) and DSPEC. The gain on DSPEC was set and matched to that of MSI-DSP and MMI-DSP(5) in the way that all spectra were aligned. As is apparent visually, all 3 spectra were consistent and MMI-DSP(5) functionality is as satisfactory as MSI-DSP. 


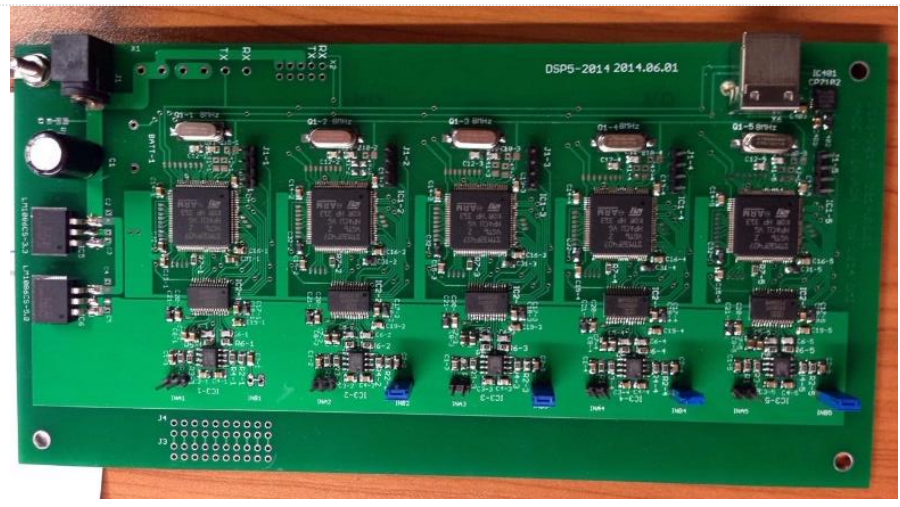

a.

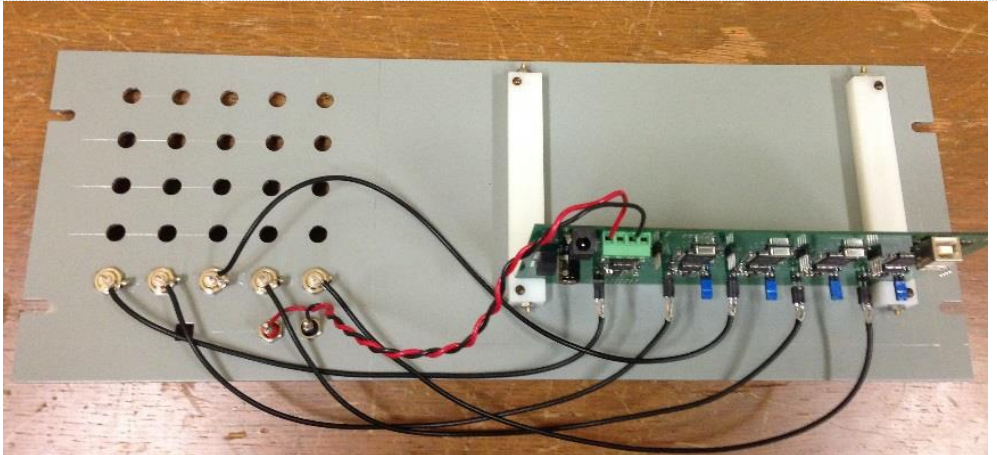

b.

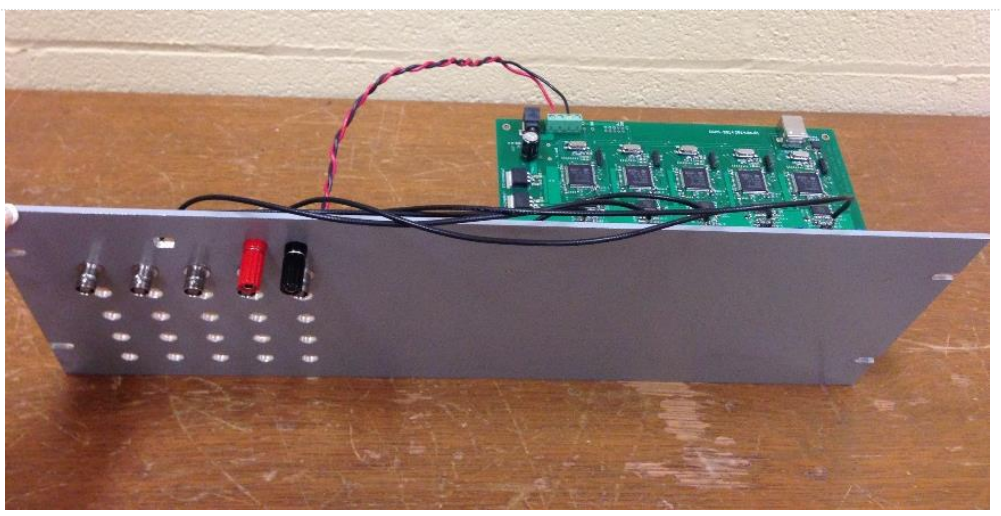

c.

Fig. 3.14 a. A board of MMI-DSP consists of 5 channels, b. Wiring and assembly of the board on a 19- inch rack panel (top view), c. Front view, showing the BNC and power connections 


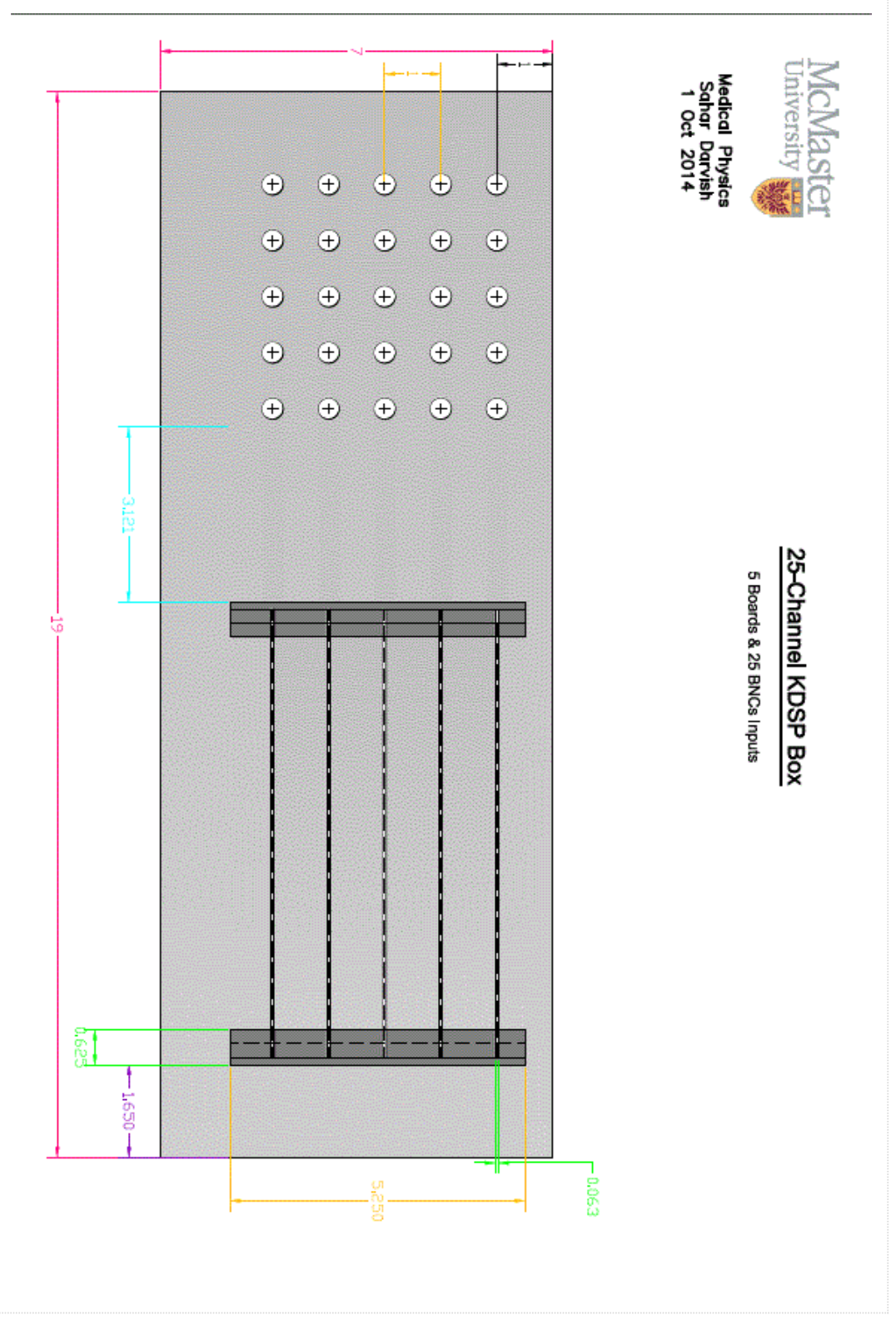

Fig. 3.15 Design drawing of MMI-DSP mounting panel 


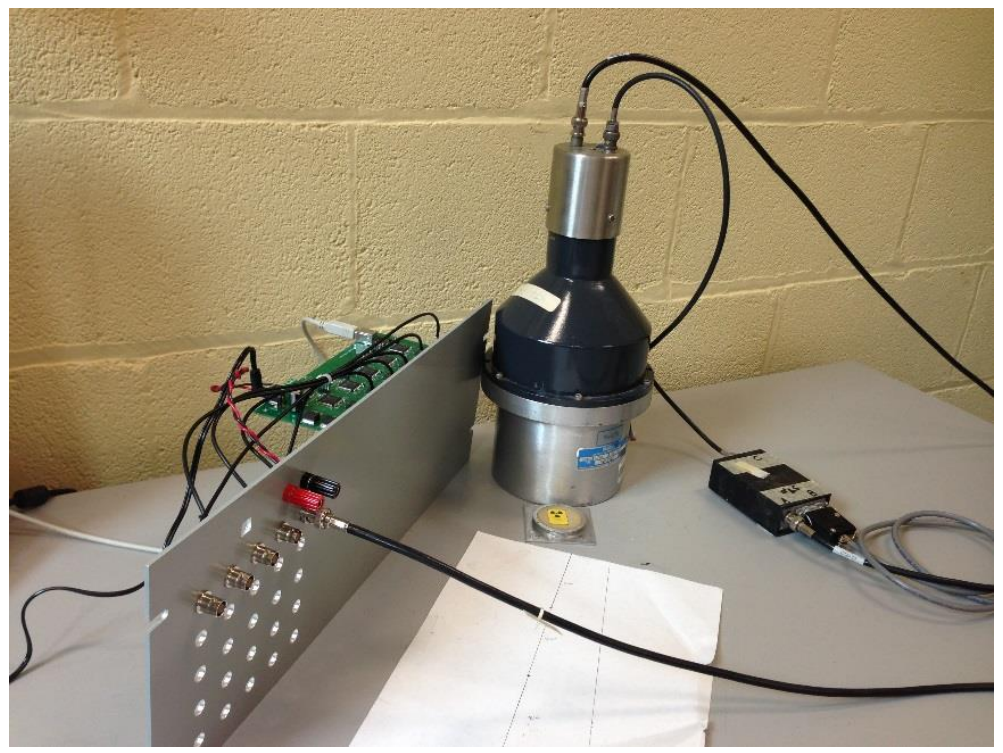

Fig. 3.16 Experimental setup for testing MMI-DSP(5) 5-channel

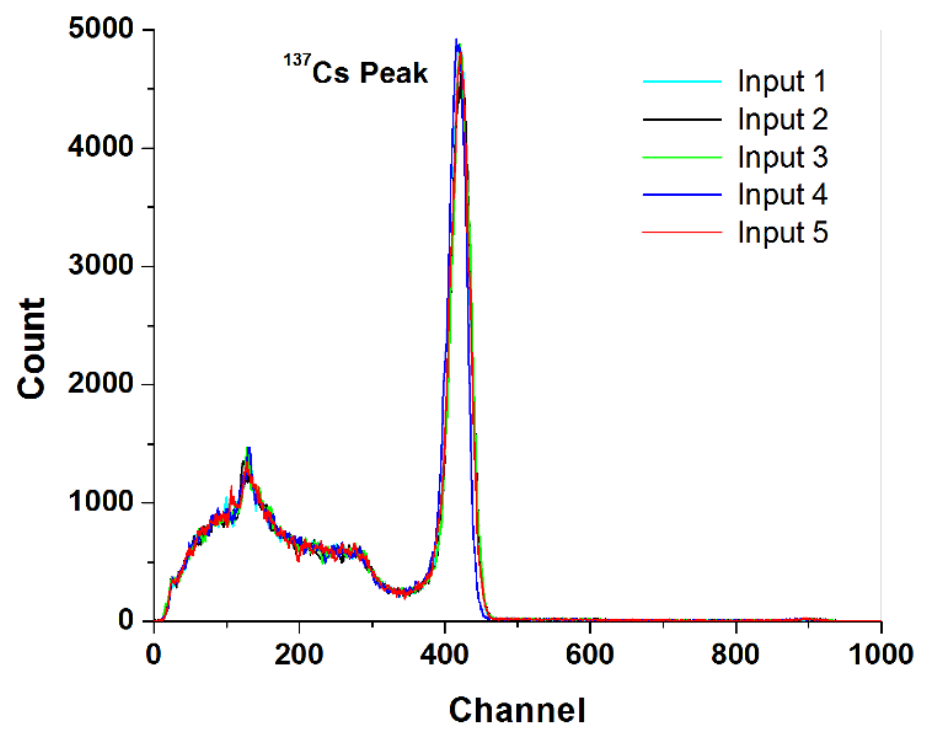

Fig. 3.17 ${ }^{137} \mathrm{Cs}$ gamma spectrum using MMI-DSP(5) 


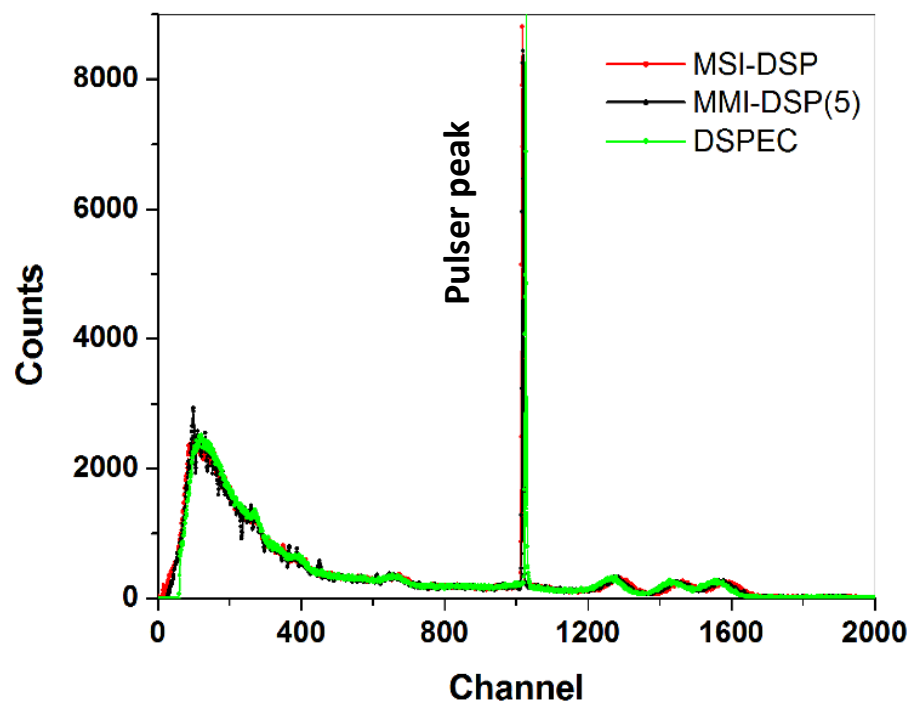

a.

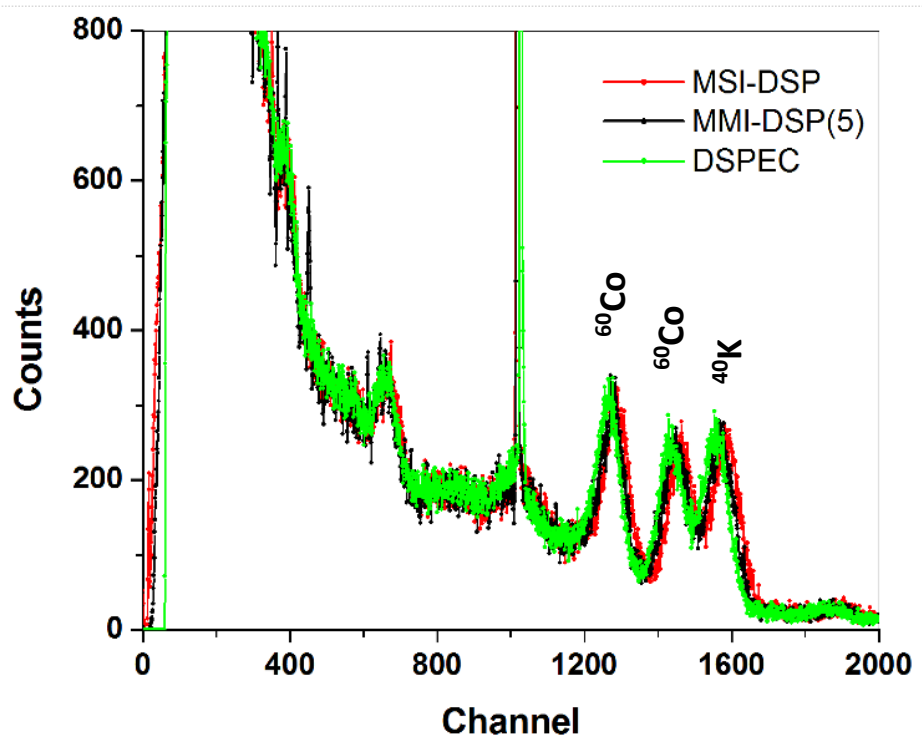

b.

Fig. 3.18 a. ${ }^{60} \mathrm{Co}$ gamma spectrum with a pulser peak at channel 900 using MSI-DSP, MMI-DSP(5) and DSPEC, b. Zoom in of spectra 
Next, the second board consisting of 5 DSPs was assembled and wired (see Fig. 3.19). The power of the second board is provided by connecting to the power pins on the first board. Also, there is no USB connector on the subsequent boards, and all boards are essentially connected to the acquisition system through the USB connector on the first board. The controlling program, in MATLAB, was also changed accordingly to control 10 DSPs simultaneously.

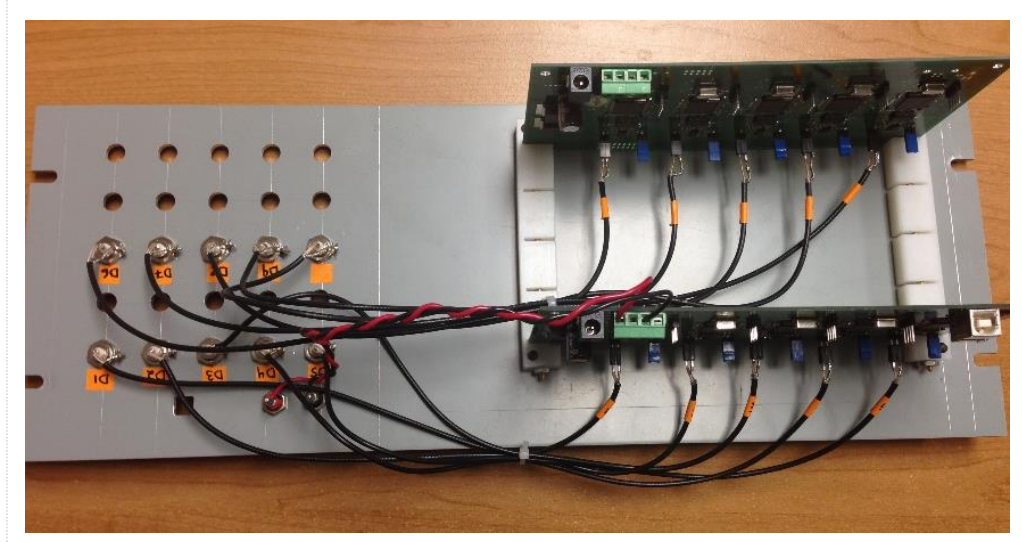

a.

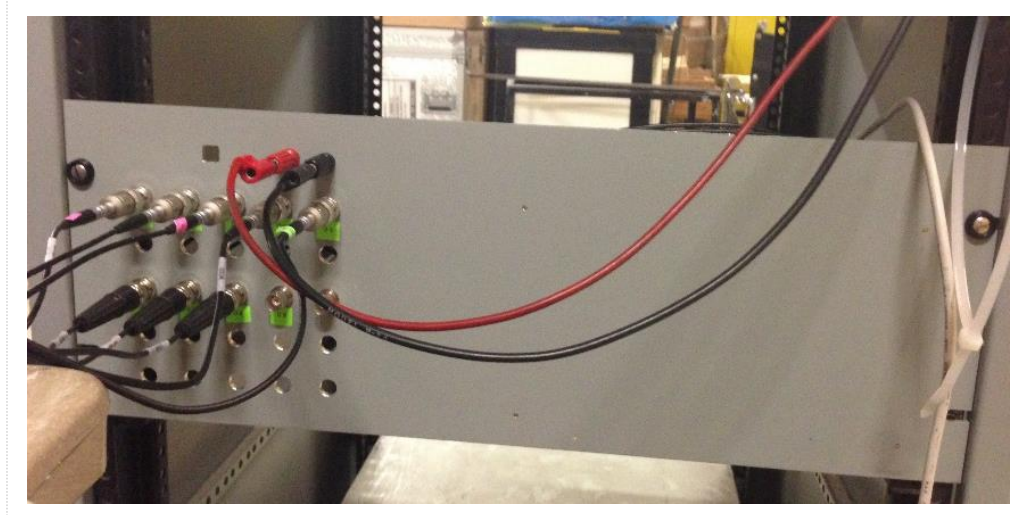

b.

Fig. 3.19 a. Assembly and wiring of MMI-DSP(10), b. Front panel view 


\subsection{Summary}

Most, if not all, commercial DSP pulse height analyzer systems (PHA) use the fast channel and slow channel approach, the fast channel being implemented in FPGA. However, MMI-DSP does not use this approach in order to keep it simple and less costly. In addition, unlike the commercial digital PHAs, which use digital filters and trapezoidal pulse shaping to determine the pulse energy, MMI-DSP uses a simpler approach to increase the processing speed. Another advantage of the McMaster prototype DSP is its excellent pileup correction instead of pileup rejection. Ultimately, considering overall cost, size and flexibility, our custom made multi-input digital signal processing system (MMI-DSP) was the best choice for the purpose of this research and overcame one of the biggest challenges of this study, which was essentially processing 9 detector signals simultaneously. 


\section{Chapter 4}

\section{Experimental Methodology}

4.1 Neutron Irradiation Facility

4.2 Experimental Setup

4.2.1 Single gaseous cavity: Preliminary neutron measurements with commercial DSP

4.2.2 Single gaseous cavity: Preliminary neutron measurements with prototype MSI-DSP

4.2.3 $3 \times 3$ cavity array: Neutron measurements with MMI-DSP 


\section{Experimental Methodology}

In this chapter, experimental setups for neutron measurements will be described in detail. The neutron source, which was used through out all measurements, as well as measurement procedures, will be explained.

\subsection{Neutron irradiation facility}

All experiments were conducted using the Tandetron Accelerator at McMaster University. It has a 1.25 MV terminal voltage (hence a total of 2.5 MV accelerating voltage) and a $0.8 \mathrm{~mA}$ capability. Fig. 4.1 shows the floor plan of the Tandetron Accelerator lab. There are two sections: 1 ) irradiation area, where the accelerator itself, irradiation box and detector were located, 2) a shielded control room where the operator and most of the electronics and acquisition systems were situated.

Fig. 4.2 shows a layout of the neutron irradiation facility located in irradiation area. The irradiation facility was built for both medical and radiobiological applications and a full description of its design principle was reported by (Pejovic'-Milic' et al, 2006; Byun et al, 2007). Fast neutrons are produced via the ${ }^{7} \mathrm{Li}(\mathrm{p}, \mathrm{n})$ reaction and then moderated by $2.4 \mathrm{~cm}$ thick polyethylene sheets. In order to reduce the radiation dose from the gamma-rays emitted from the Li target $(478 \mathrm{keV}), \mathrm{Li}\left(\mathrm{p}, \mathrm{p}^{\prime} \gamma\right)$, and from the moderator $(2.2 \mathrm{MeV}), \mathrm{H}(\mathrm{n}, \gamma)$, a lead filter is positioned right after the polyethylene moderator. A graphite reflector surrounding the irradiation site reflects neutrons back to the irradiation site, which increases the neutron fluence rate at the irradiation site. A cylindrical cavity volume of 20 $\mathrm{cm}$ diameter and $6 \mathrm{~cm}$ length is used for positioning the irradiation object of interest. The boron plastic walls and the lead absorb the outgoing neutrons and gamma-rays, respectively. The neutrons transmitting the boron plastic wall are absorbed by the shield box made of Borax, polyethylene and polyester resin. To allow the access of a subject's hand, there is a hole along the lateral axis. Therefore the radiation field of the irradiation facility consists of three different regions:

1) Inside the cavity where the palm of the hand is placed,

2) The arm access hole (in Y direction) 
3) Larger size cavity for irradiation of bigger objects like my detector (in X direction) 4) Outside the shielding box.

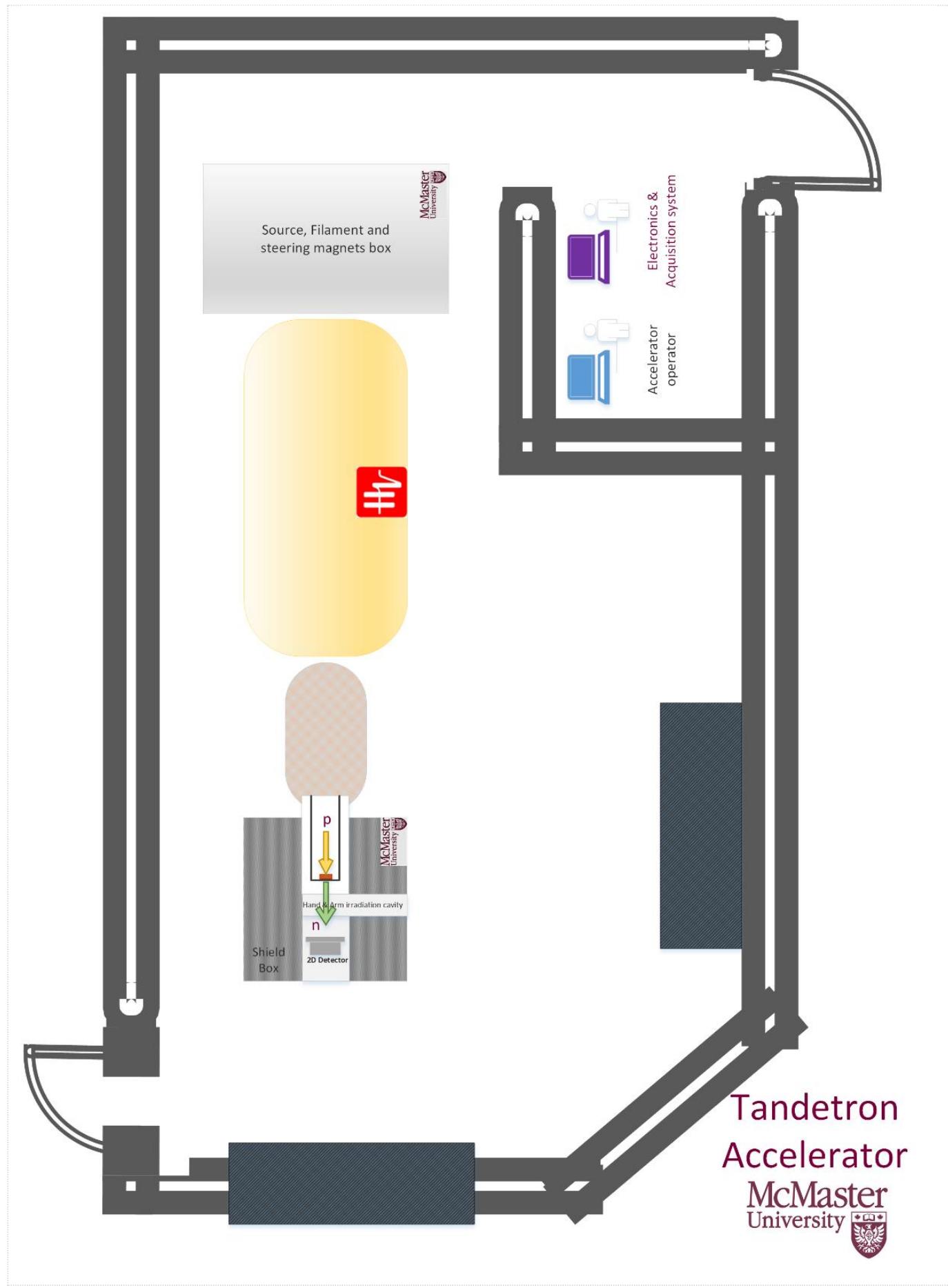

Fig. 4.1 Tandetron Accelerator floor plan 


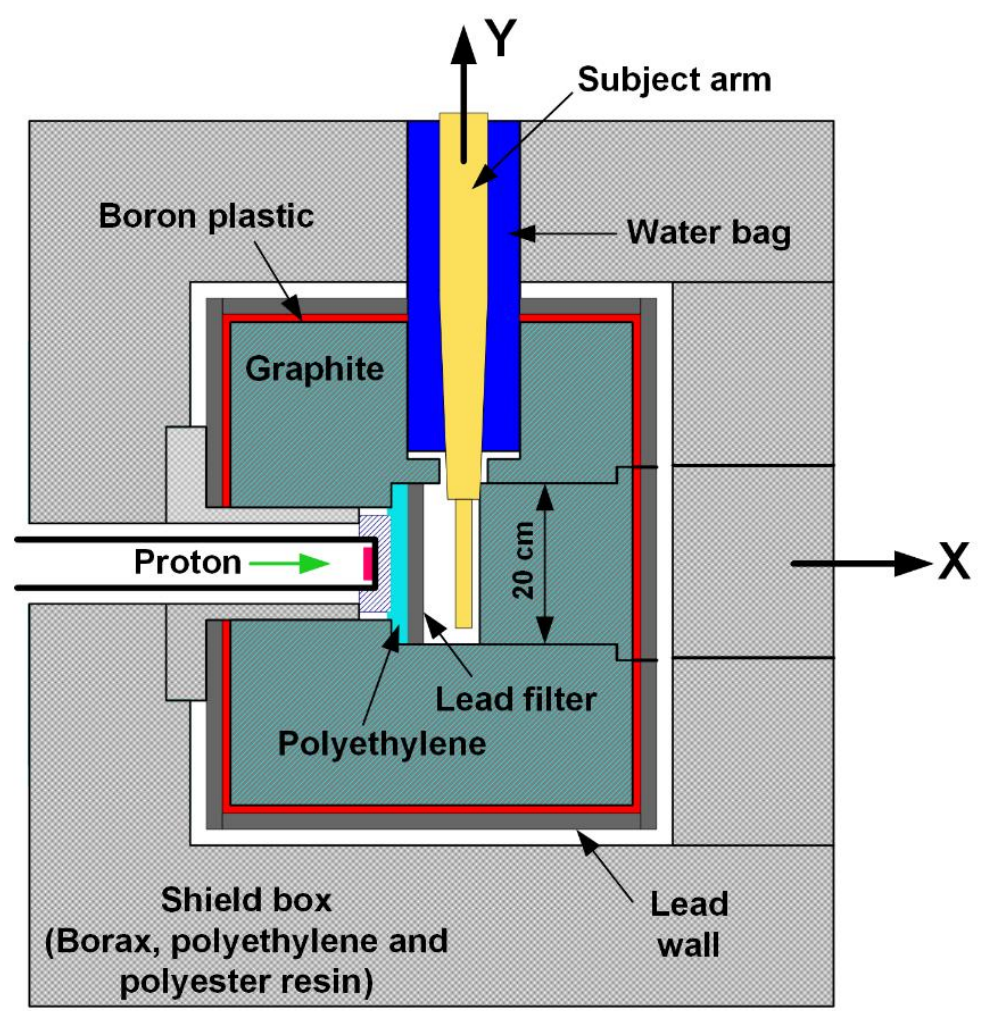

Fig. 4.2 Layout of the Tandetron neutron irradiation facility at McMaster University (Byun et al, 2007)

The overall dimension is $127 \times 130 \times 122 \mathrm{~cm}^{3}$. The detailed characteristics of the radiation field and a comprehensive dosimetric characterization of the irradiation cavity, measured by the commercial 1/2" TEPC will be discussed in chapter 6 .

\subsection{Experimental setup}

As was mentioned in the previous section, there are two approaches to test the fabricated 2D THGEM detector. 1) To test the performance of the detector and to be assured that all fabricated parts, wire solderings, connections and vacuum seals are properly done. Preliminary measurements were carried out using single a volume configuration (center detector) and commercial digital signal processing system. 2) Once satisfactory results were obtained, all 9 detectors were tested simultaneously using a 2D multi-volume configuration and custom made multi input digital signal processing system. In the next two subsections the experimental setup of each approach will be explained thoroughly. 


\subsubsection{Single gaseous cavity: Preliminary neutron measurements with commercial DSP}

Due to the detector size, using the hand and arm access cavity is impractical. Hence the THGEM detector was placed inside the irradiation cavity opening at the other side (along the $\mathrm{X}$ axis), centerd on the beam path as shown in Fig. 4.3 and $4.4 a$.

A Canberra dual high voltage supply (Model 3125, the range of each module: 0 to $\pm 5000 \mathrm{~V}$ ) with two independent outputs was connected to SHV connectors on the detector to bias the cathode and THGEM individually and were labelled as cathode HV and THGEM HV to avoid any confusion. Negative high voltage values were applied to the cathode and THGEM and the anode was kept at ground potential.

Using a short coaxial signal cable, RG-62/U, the detector was connected to the input of an ORTEC charged sensitive preamplifier (Model A190). The preamplifier output was connected to the input of an ORTEC digital signal processor (DSPEC) which is placed in the control room about $15 \mathrm{~m}$ away, across the accelerator hall (see Fig. 4.1). The output of the DSPEC was then connected simultaneously to the laptop for data acquisition and to the ORTEC amplifier for observing and monitoring the preamplifier and amplifier signals on the oscilloscope. DSPEC is fully supported and remote-controlled by a laptop with MAESTRO software for the data acquisition. The test input of the preamplifier was connected to the pulser placed in the NIM bin of electronics in the control room to test the functionality of the preamp as well as calibrating the detector signal (to verify the gain differences between each measurement).

Once the experimental setup was completed on the irradiation cavity side, all the interlock systems were activated and everything was ready to operate the accelerator with the desired settings. Fig. $4.4 \mathrm{~b}$ and $c$ illustrate the control room, accelerator operator desk and NIM bin of the electronics. The mixed neutron and gamma-ray field was generated using a proton beam with different energies of $2 \mathrm{MeV}, 2.3 \mathrm{MeV}$ and $2.5 \mathrm{MeV}$ and various proton currents of $50 \mu \mathrm{A}, 150 \mu \mathrm{A}$ and $250 \mu \mathrm{A}$. 


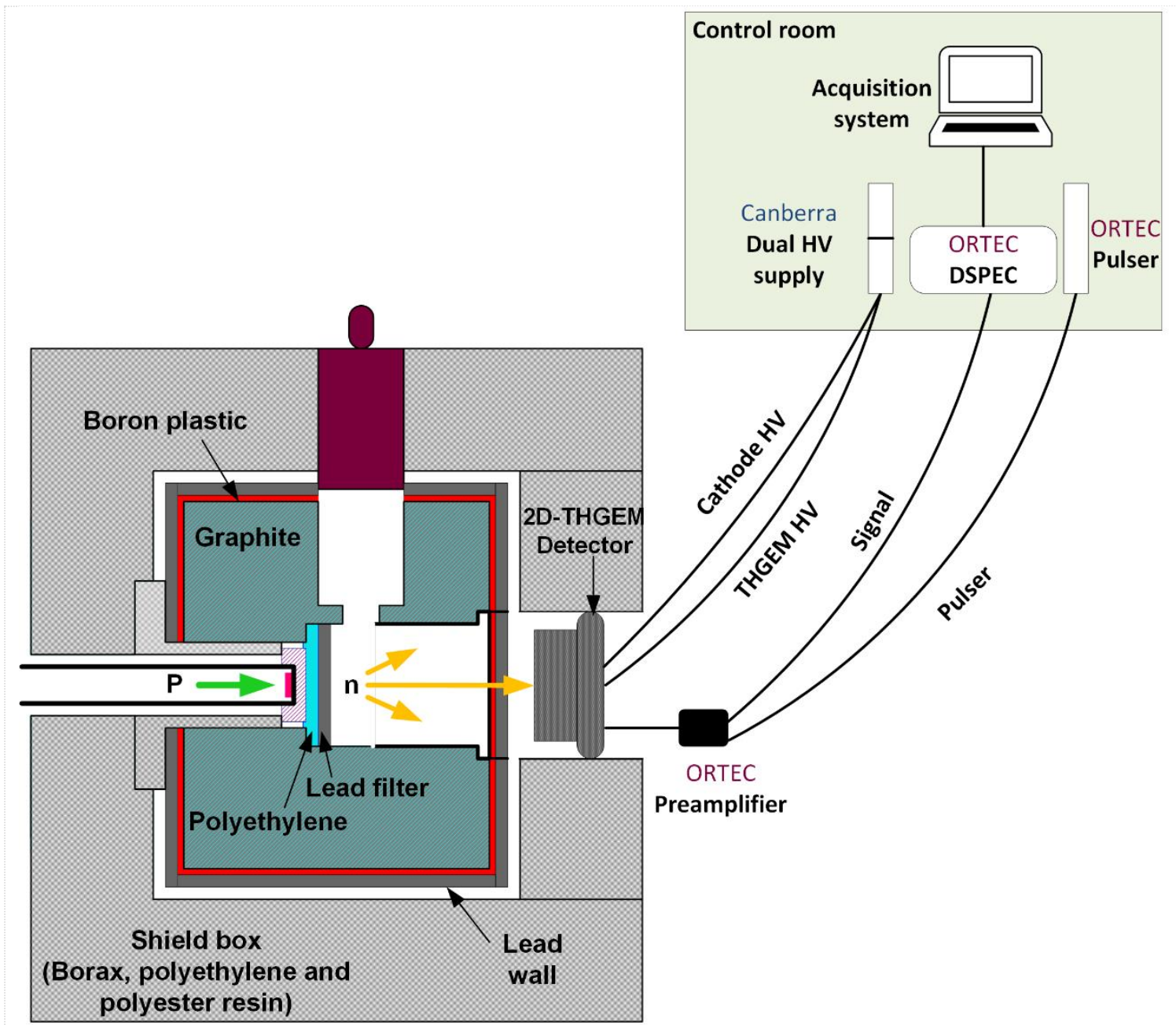

Fig. 4.3 Schematic of experimental setup for single volume detector configuration 


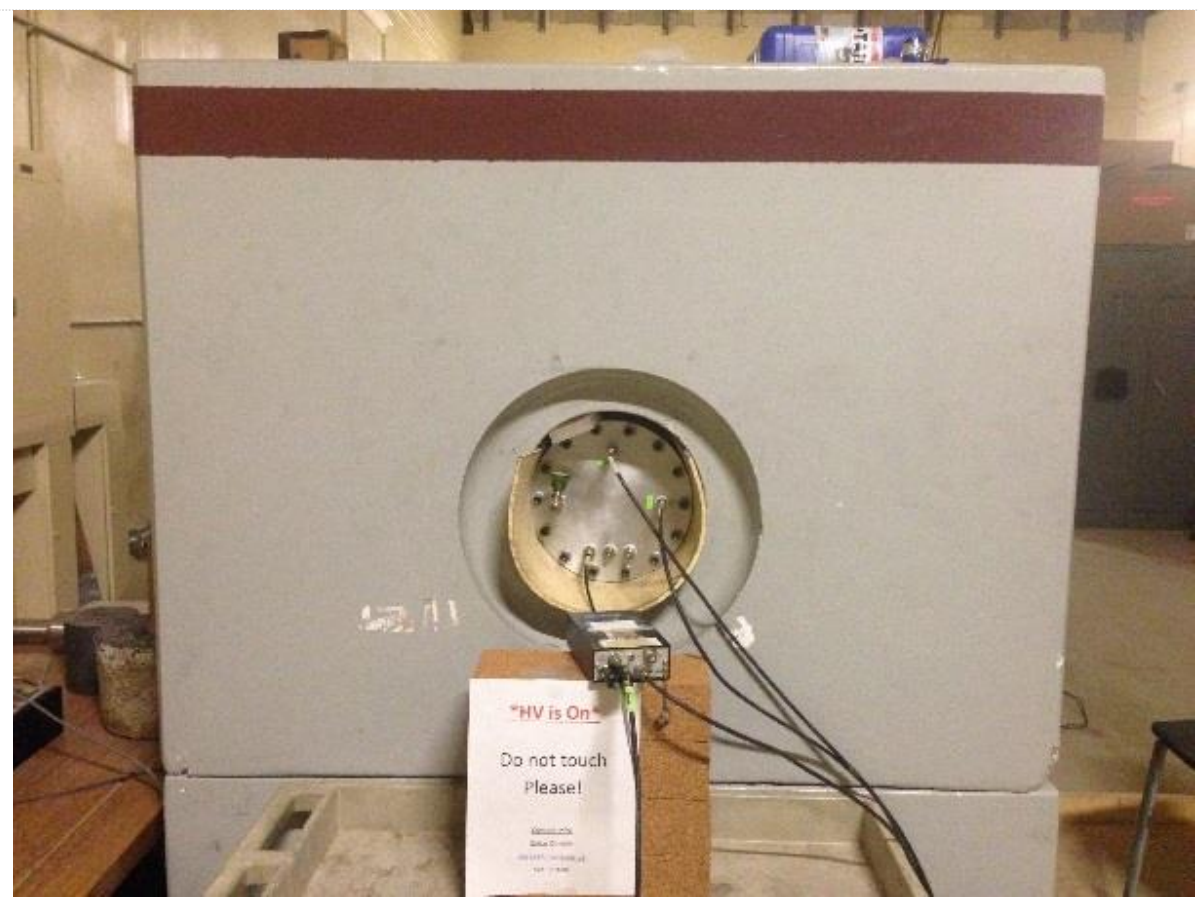

a.

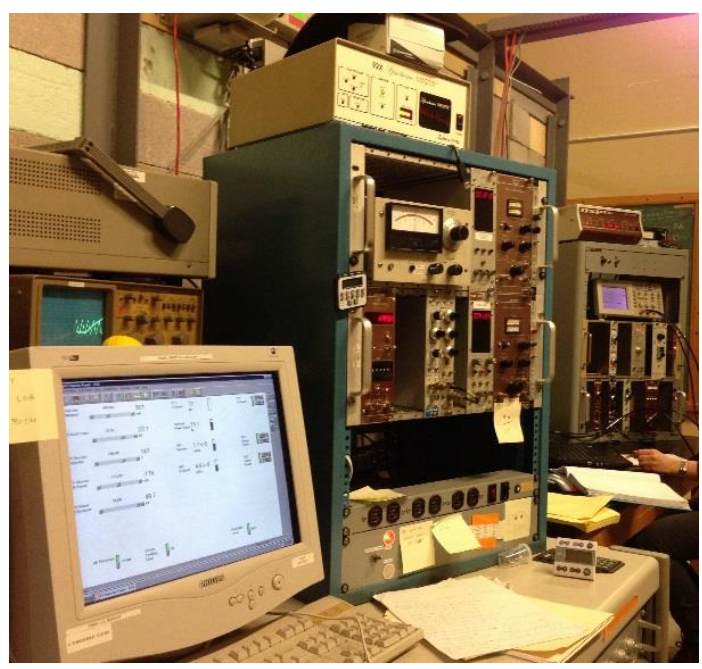

b.

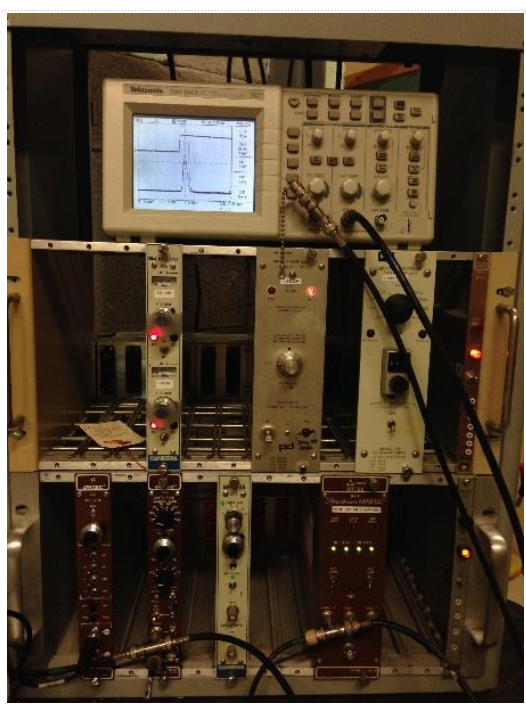

c.

Fig. 4.4 a. Single volume detector placed in the irradiation cavity connected to the ORTEC charge sensitive preamplifier, b. Control room: accelerator operator desk and electronics c. electronics NIM bin: dual HV, pulser, amplifier 


\subsubsection{Single gaseous cavity: Preliminary neutron measurements with prototype MSI-DSP}

Once it was verified that the single THGEM detector was working properly and a high voltage operating range was determined, it was time to test the prototype MSI-DSP for neutron measurement and compare its performance to the commercial one. To this end the same experimental setup which was explained in the previous subsection was used, except that the commercial ORTEC signal processing system (DSPEC) was replaced by the prototype MSI-DSP (refer to chapter 3, section 3.1.1) (see Fig. 4.5).

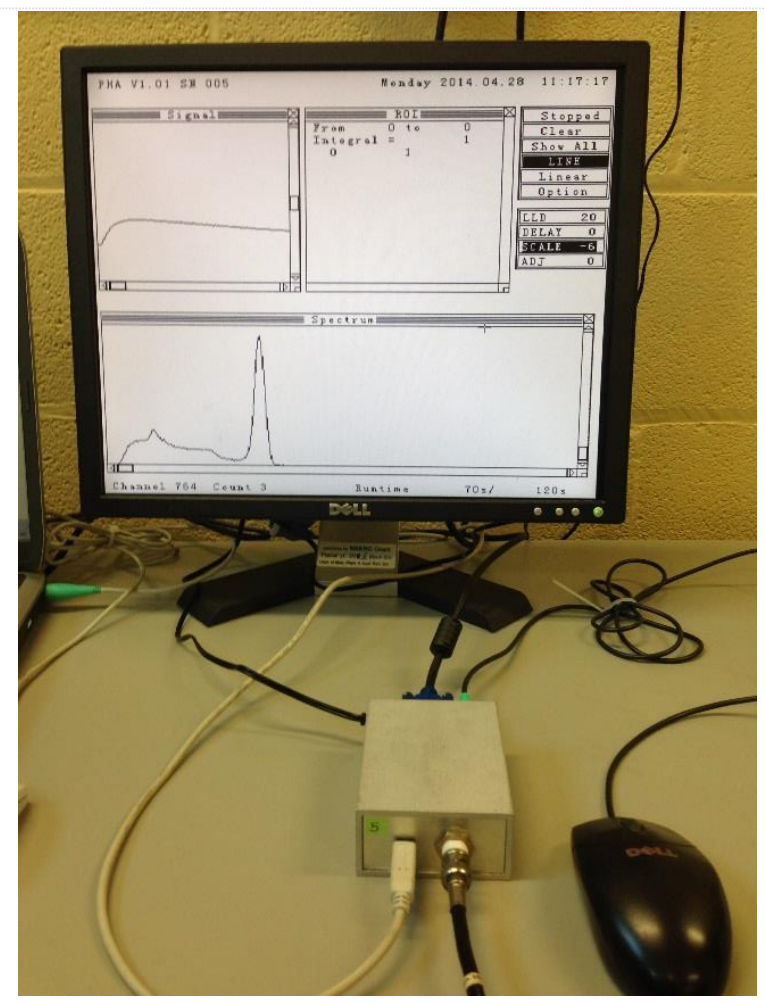

Fig. 4.5 Prototype custom made single input DSP (MSI-DSP) 


\subsection{3 $3 \times 3$ gaseous cavity array: Neutron measurements with MMI-DSP}

After testing both a single volume detector and the prototype MSI-DSP, the final phase of the research was launched. In this phase, the baseplate was switched to the multi-pin configuration and all the 9 detectors were in play as was described in section 2.7.2. There was a challenge for the experimental setup in this part which was how to connect 9 signal cables plus two pulser cables, as the control room was $15 \mathrm{~m}$ away from the detector side. There were two possibilities:

1) Keep the signal processing system (MMI-DSP) in the control room: In this case, MMI-DSP would easily connect to the acquisition system via a short USB cable. However, the 9 signal outputs of the preamplifier, plus 2 test inputs have to be connected to the MMI-DSP in the control room via $15 \mathrm{~m}$ cables.

2) Keep the signal processing system (MMI-DSP) close to the detector, in the irradiation area: In this case, the 9 signal outputs of the preamplifier, plus 2 test inputs would be connected to the MMI-DSP via 11 short $(1 \mathrm{~m})$ cables. To connect MMI-DSP to the acquisition system which is in the control room, a $15 \mathrm{~m}$ USB cable was required.

The second method has the advantage of avoiding 9 long signal cables $(15 \mathrm{~m})$ connected to the MMI-DSP across the Accelerator hall and just one $15 \mathrm{~m}$ signal transfer cable would go to the control room for signal acquisition. As the longest USB cable available in the market was maximum $5 \mathrm{~m}$, I decided to use a type pf extension cable. Hence, USB2 extension cable was purchased (iCAN, model USB2 GW-AR2-15M) and tested. The same results were obtained in terms of communication between MMI-DSP and laptop compared to the $1 \mathrm{~m}$ USB cable. Thus, this method was chosen for experimental setup as is shown schematically in Fig. 4.6. The actual experimental setup is demonstrated in Fig. 2.7.

For this setup, a brand new low noise charged sensitive preamplifier was purchased from CAEN which has 8 BNC inputs and 8 LIMO outputs (Model A1422(8ch)). For the $9^{\text {th }}$ detector a single unit CAEN preamplifier was purchased (Model A1422(1ch)). The cables were prepared as was explained in section 2.7.2. 


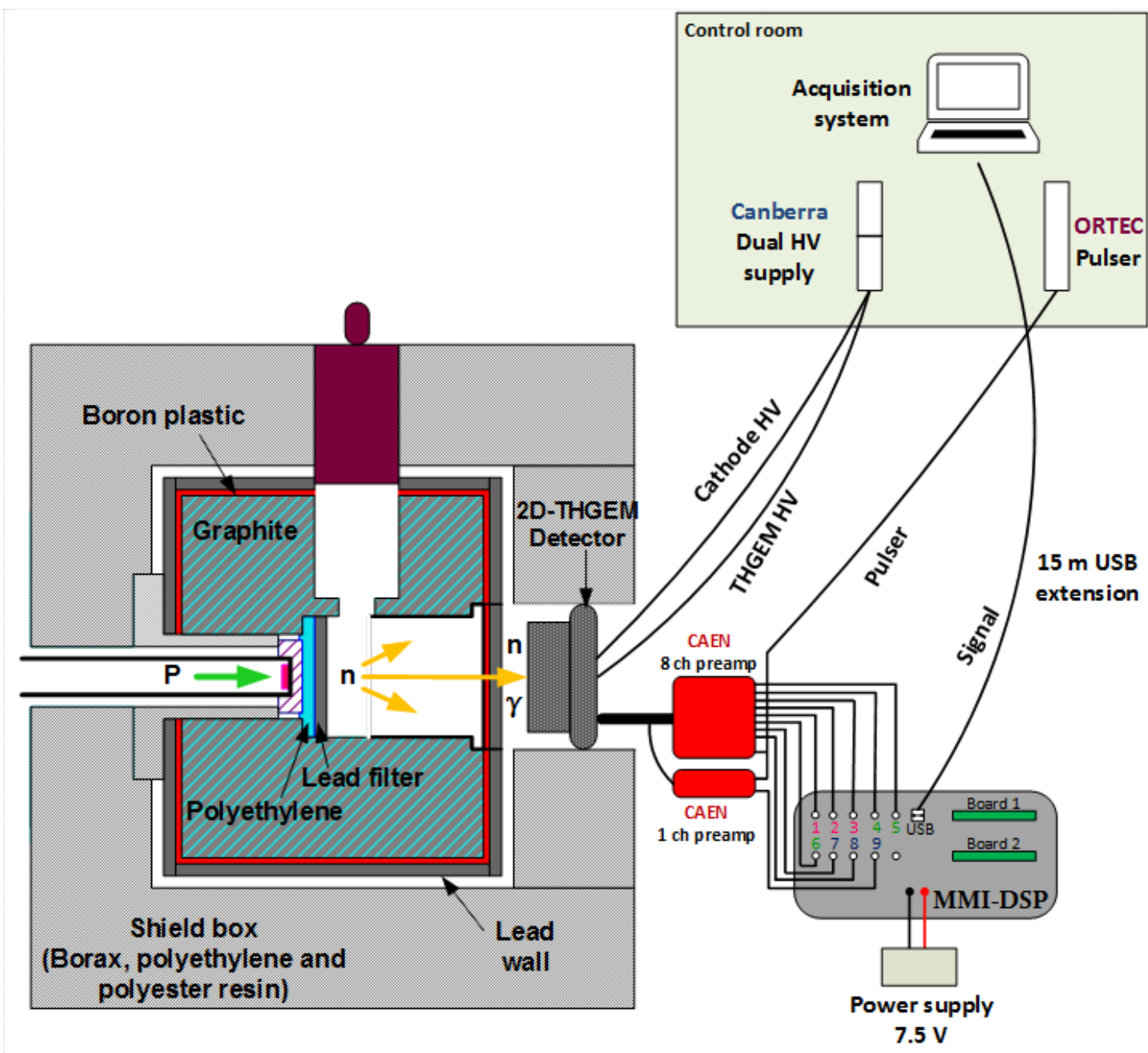

Fig. 4.6 Schematic of experimental setup for multi-volume detector configuration 


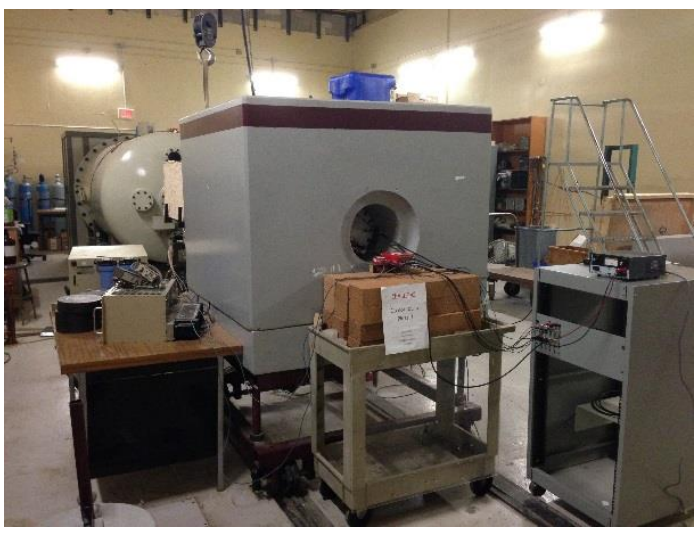

a.

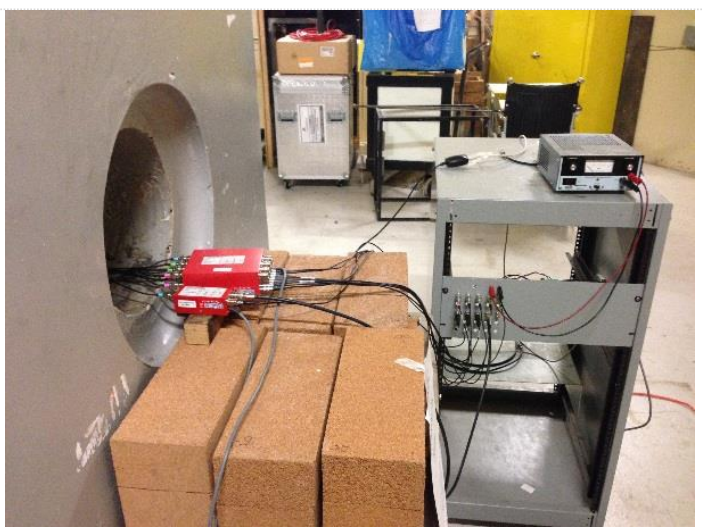

c.

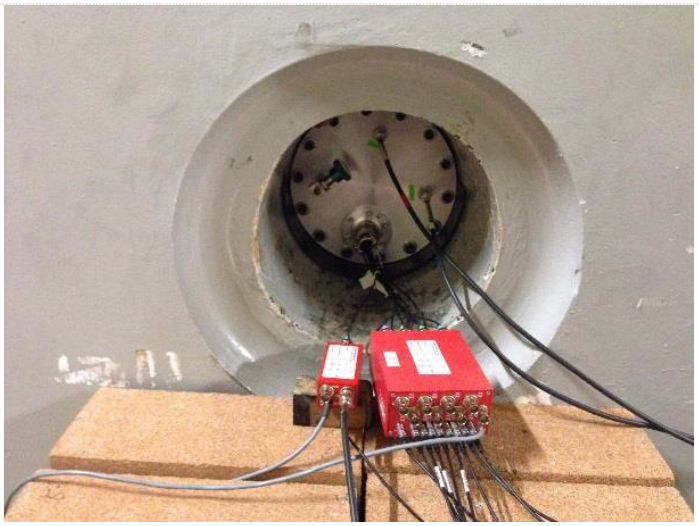

e.

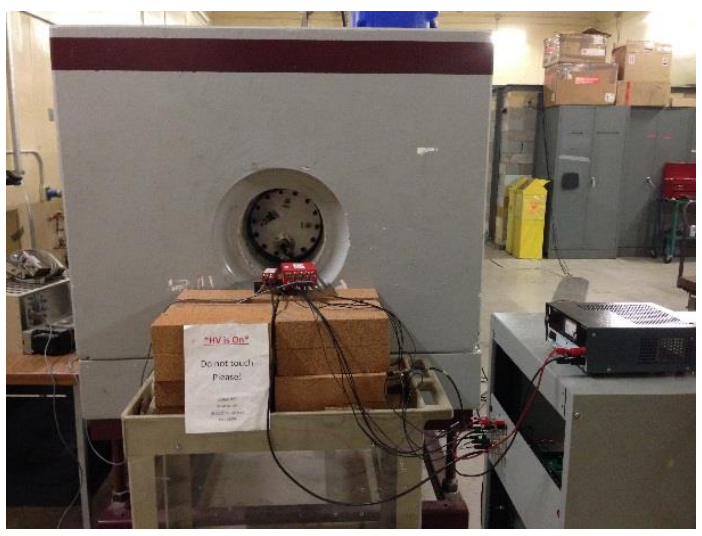

b.

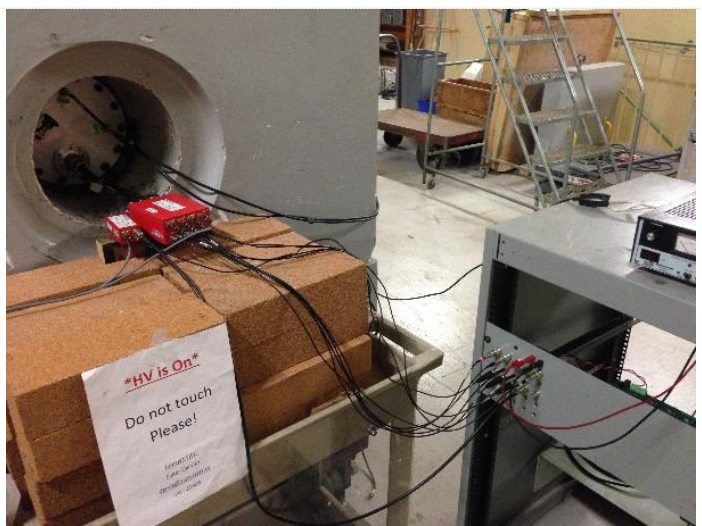

d.

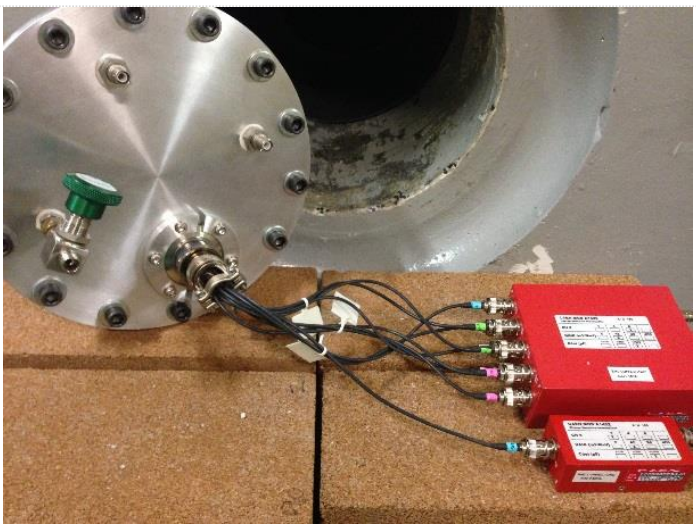

f.

Fig. 4.7 Experimental setup for multi-volume detector and multi-input MMI-DSP: 2D THGEM detector is placed in the irradiation cavity and connected to CAEN 8 channels and 1 channel charge sensitive preamplifier 


\section{Chapter 5}

\section{Data Analyses Methodology}

5.1 Data Analysis Methodology for commercial 1/2" TEPC

5.2 Data Analysis Methodology for 2D THGEM Detector 


\section{Data Analysis Methodology}

In this chapter, data analysis procedures will be described for data collected using commercial 1/2" TEPC and 2D THGEM detector.

\subsection{Data analysis Methodology for $1 / 2$ " TEPC}

After data collection is done, each TEPC spectrum with 16k channels was calibrated in terms of the lineal energy by applying the appropriate calibration factors obtained from the internal alpha source calibration. A finely collimated internal ${ }^{244} \mathrm{Cm}$ alpha source deposits $170 \mathrm{keV}$ energy (ICRU 1983) for a $2 \mu \mathrm{m}$ diameter simulated sphere of tissue, which corresponds to a lineal energy of $127 \mathrm{keV} \mathrm{\mu m}^{-1}$. By fitting the measured alpha peak with a Gaussian curve as shown in Fig. 5.1, the corresponding channel number of the alpha peak was found. Having the alpha peak channel number, together with the pulser calibration result, i.e. a linear relation between pulser amplitude and channel number as shown in Fig. 5.2 , each channel number was converted to corresponding lineal energy.

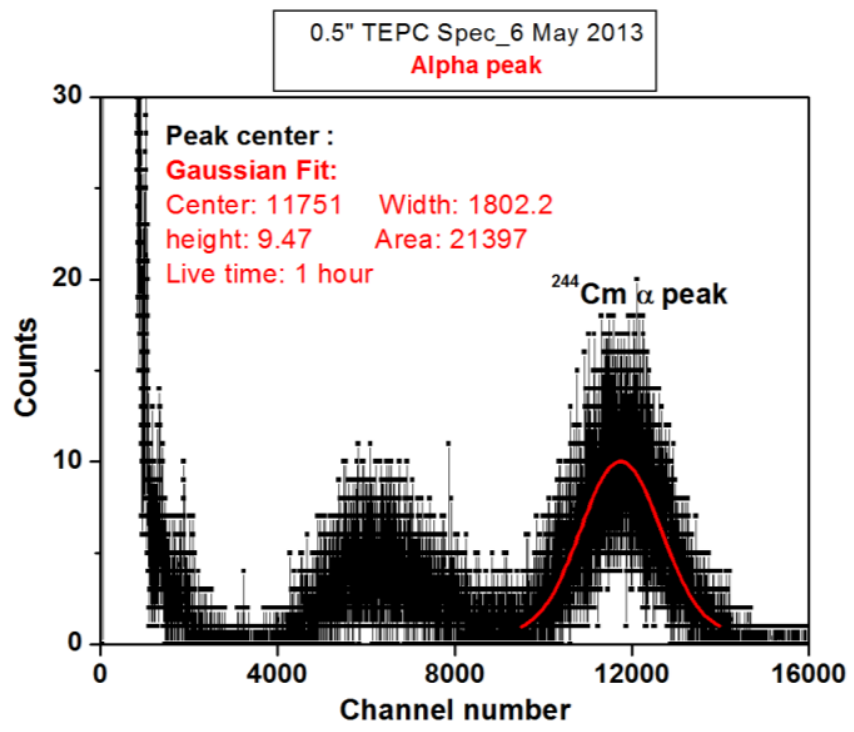

Fig. 5.1 ${ }^{244} \mathrm{Cm}$ internal Alpha source spectrum with Gaussian peak fit 


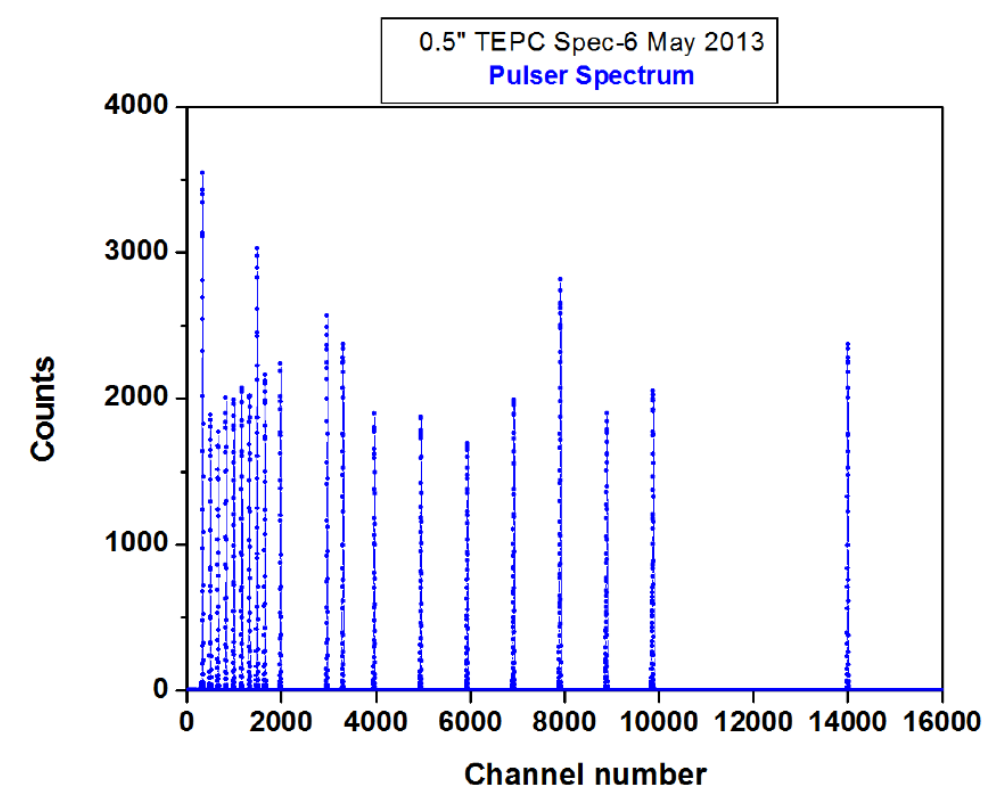

a.

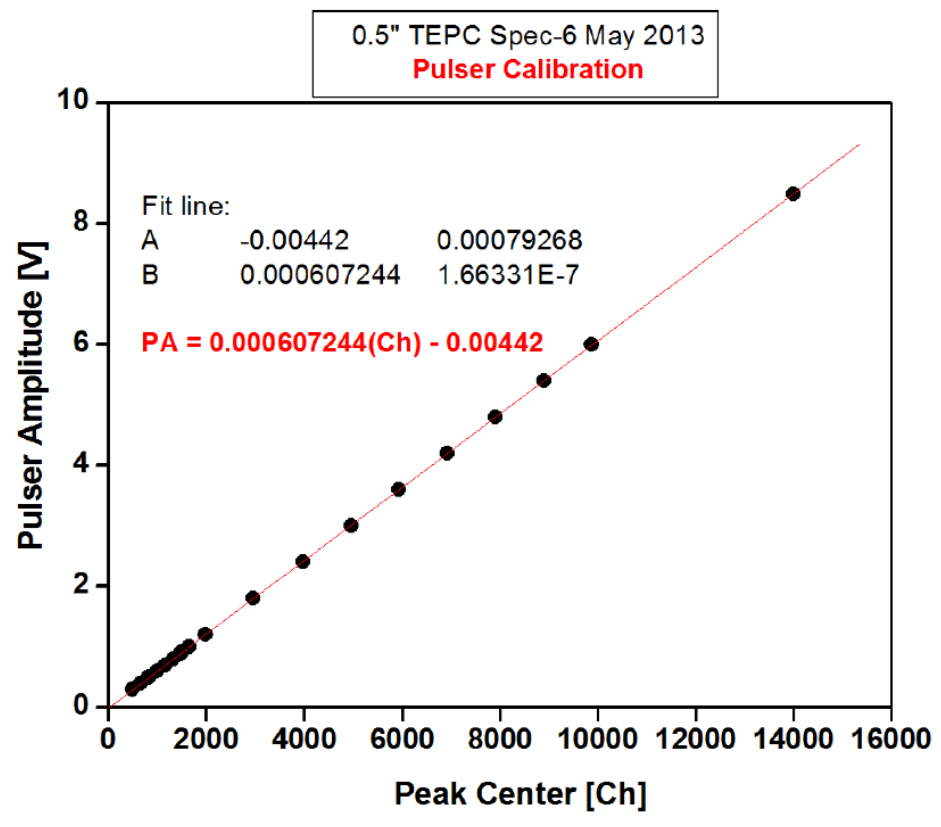

b.

Fig. 5.2 a. Pulser spectrum, b. Pulser calibration 
After lineal energy calibration using eq. 5-1 and a conversion code written in MATLAB, each spectrum was redistributed into equal logarithmic bins with a resolution of 60 bins per decade, and then $\mathrm{N}\left(\mathrm{y}_{\mathrm{i}}\right)$, the number of counts in a logarithmic bin of lineal energy $\mathrm{y}_{\mathrm{i}}$, was multiplied with the lineal energy to represent a dose spectrum, from which the neutron and gamma-ray absorbed doses were calculated. Origin 6.0 software was employed for data analysis in which all spectra throughout the thesis were plotted.

$$
y_{i}=P A_{i}\left(\frac{127}{P A_{127}}\right) \quad k e V \mu m^{-1}
$$

Where $y$ denotes lineal energy in unit of $\mathrm{keV} \mu \mathrm{m}^{-1}, P A_{i}$ corresponds to the pulse amplitude of channel $i, P A_{127}$ is the pulse amplitude of alpha peak and 127 is the lineal energy of the internal ${ }^{244} \mathrm{Cm}$ source inside the spherical TEPC.

Typical raw and redistributed spectra are illustrated in Fig. $5.3 a$ and $b$ respectively. The area under the spectrum is related to the absorbed dose (D) within the sensitive volume of the detector given by:

$$
D=C \sum_{i} y_{i} N\left(y_{i}\right) \quad[m G y]
$$

where the summation represents the area under the spectrum and $C$ is given as:

$$
\mathrm{C}=1.602 \times 10^{-16} \frac{\bar{l}}{\rho V}
$$

$\bar{l}$ is the mean cord length, $\rho$ is the density of the gas, $V$ is the sensitive volume of the detector and $1.602 \times 10^{-16}$ is the conversion factor from $\mathrm{keV}$ to $\mathrm{J}$. In other words, according to the definition of absorbed dose, $\rho V$ is the mass of the tissue equivalent gas within the sensitive volume that can be calculated for any given detector size. Mass of each detector that will be used in this thesis was calculated and tabulated in table 5-1. To simulate $2 \mu \mathrm{m}$ diameter of tissue, $\mathrm{C}$ was calculated for each detector size accordingly and presented in table 5-1. 
Table 5-1. Mass and dose conversion coefficients for each detector

\begin{tabular}{cccccc}
\hline Detector & $\begin{array}{c}0.5 ” \\
\text { TEPC }\end{array}$ & 2” TEPC & \multicolumn{3}{c}{ 2D THGEM } \\
\cline { 4 - 6 } $\begin{array}{c}\text { Mass } \\
{[\mathrm{mg}]}\end{array}$ & 0.1689 & 3.4040 & 0.0393 & 0.0098 & 0.0017 \\
\hline $\begin{array}{c}\mathrm{C} \\
\times 10^{-7}\left[\mathrm{~J} \mathrm{~m} \mathrm{keV}^{-1} \mathrm{~kg}^{-1}\right]\end{array}$ & 12.6 & 0.628 & 54.39 & 218 & 1256.5 \\
\hline
\end{tabular}

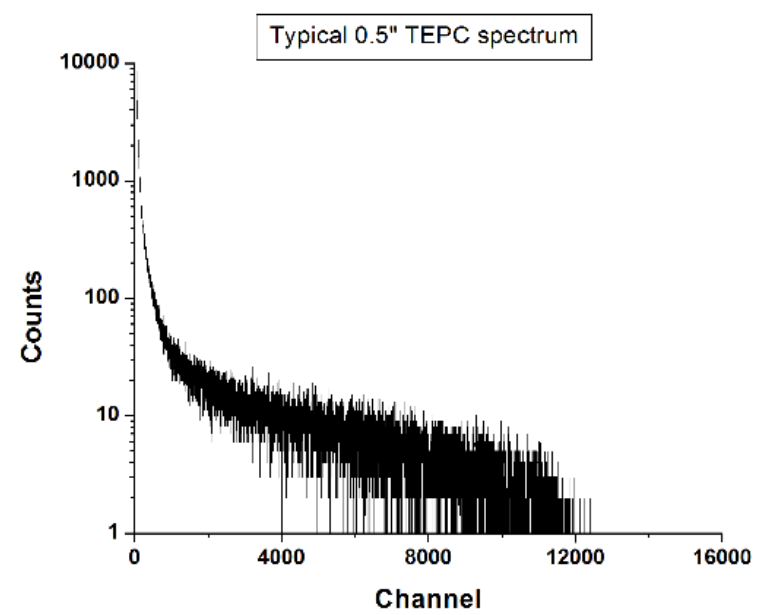

a.

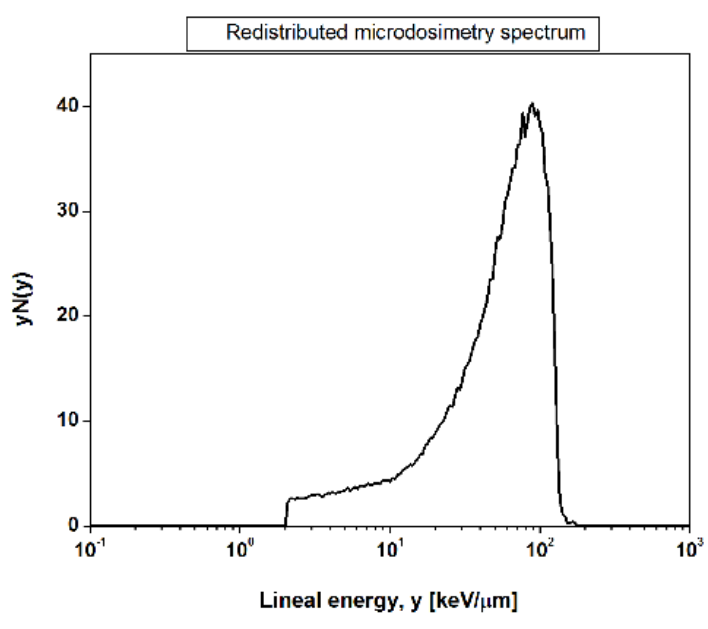

b.

Fig. 5.3 Typical 1/2" TEPC a. raw spectrum, b. microdosimetric spectrum 
Dividing the absorbed dose by the related proton charge of each measurement, the

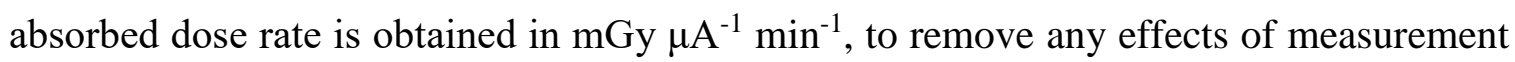
setting changes. Also, doses for an arbitrary set of proton current and irradiation time can be easily scaled.

\subsection{Data analysis Methodology for 2D THGEM Detector}

The data analysis procedure for the 2D THGEM detector is mostly the same as the one explained above for the commercial 1/2" TEPC, except that the 2D THGEM detector does not have any internal calibration source, making the lineal energy calibration trickier. In this case, according to Kliauga (1990) a reliable and accurate calibration method is based on the known proton peak or proton edge.

Taking the advantage of the fact that the lineal energy of the neutron peak and proton edge is the same for each proton energy, once the spectrum obtained with the 1/2" TEPC is calibrated, the lineal energy corresponding to the neutron peak can be adopted to calibrate the spectrum obtained with 2D THGEM detector. The rest of the data analysis would be the same as explained above for 1/2" TEPC.

In the case of the $3 \times 32 \mathrm{D}$ THGEM detector, there would be 9 simultaneous measurements and thus 9 pulser calibrations. Besides, in the prototype MMI-DSP, there is not an option of quick real-time analysis, and spectra are saved as a text file. Thus it is extremely time-consuming to do pulser analysis one by one. Hence, a simple code was written in MATLAB to analyze each pulser calibration, plot the data and fit the best line and print the line equation as an output shown in Fig. 5.4. 


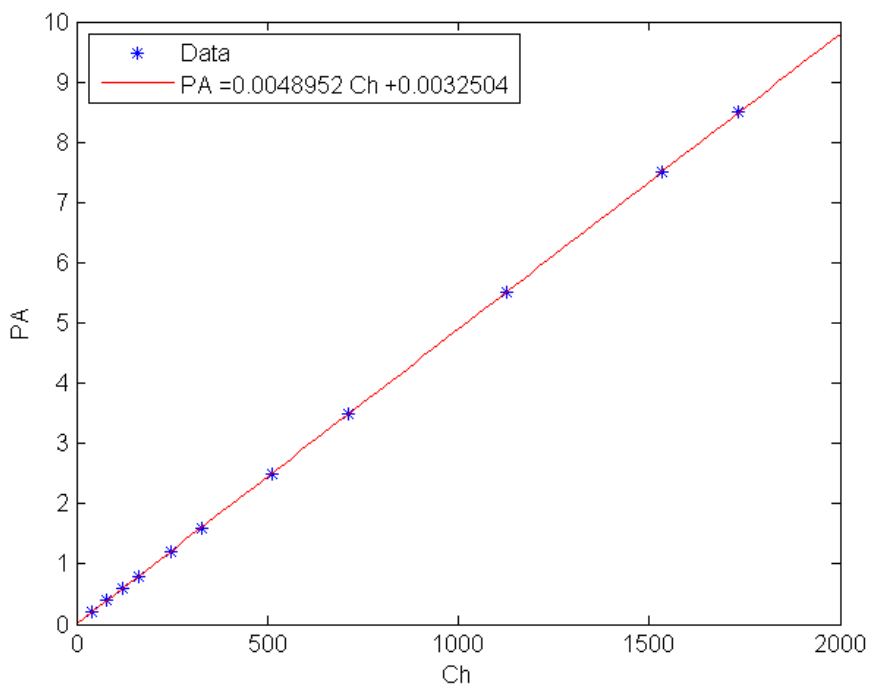

a.

Fig. 5.4 A typical MATLAB output for pulser calibration of multi-volume detector 


\section{Chapter 6}

Comprehensive Radiation Dose Measurement for the ${ }^{7} \mathbf{L i}(\mathbf{p}, \mathbf{n})$ Accelerator Neutron Field

6.1 Experimental Methodology

6.2 Results and Discussion

6.3 Conclusion 


\section{Comprehensive Radiation Dose Measurement for the ${ }^{7} \mathrm{Li}(\mathrm{p}, \mathrm{n})$ Accelerator Neutron Field}

Comprehensive dose measurement was carried out for the $\operatorname{McMaster}{ }^{7} \operatorname{Li}(\mathrm{p}, \mathrm{n})$ Tandetron accelerator neutron field. In this study, neutron and gamma-ray doses were measured for the McMaster Tandetron ${ }^{7} \operatorname{Li}(p, n)$ irradiation facility at various positions in the proton energy range of $1.95-2.5 \mathrm{MeV}$ and $50 \mu \mathrm{A}$ proton current using a tissueequivalent proportional counter (TEPC), a standard detector for a mixed neutron and gamma-ray dosimetry (Byun et al, 2007; Waker et al, 1995; Spirou et al, 2008). The irradiation facility, which was explained in section 4.2, has been intensively used for In Vivo Neutron Activation Analysis (IVNAA) to analyze trace as well as main constituent elements in the hand (Matisiyak et al, 2013; Bhatia et al, 2015).

In parallel with the absorbed dose measurements, MCNP Monte Carlo simulations were carried out by Dr. Soo Hyun Byun (Darvish-Molla et al, 2015) and from the simulation data neutron fluence spectra were computed at various positions. Subsequently, the corresponding neutron weighting factors were calculated. From microdosimetric measurements and MCNP Monte Carlo simulations, neutron and gamma-ray absorbed doses, neutron weighting factor and equivalent doses were determined for various positions in the proton energy range of $1.95-2.50 \mathrm{MeV}$ by the author.

Data accomplished in this study were accepted by the Radiation Protection Dosimetry journal in September 2015 and published online as an article in October 2015 (DarvishMolla et al, 2015).

\subsection{Experimental Methodology}

A commercial 1/2" tissue equivalent proportional counter (TEPC) (model LET-1/2, Far West Technology Inc., USA) was filled with a propane-based TE gas at a pressure of 66.4 torr, which simulates a $2 \mu \mathrm{m}$ diameter of spherical volume of tissue, and was employed for all the measurements inside the cavity and along the arm access hole (see Fig. 4.2 and 6.1). The proton current was set to $50 \mu \mathrm{A}$ and the high voltage of $600 \mathrm{~V}$ was applied to the detector. Using a built-in ${ }^{244} \mathrm{Cm}$ alpha source, the lineal energy was calibrated and the 
linearity of the system was checked using a high precision digital pulser (model PB-5, Berkeley Nucleonic Corporation, USA). The signal from the detector was processed using a commercial digital signal processor (model ORTEC DSPEC) and all the spectra were stored through a built-in Ethernet port connection to a laptop.

For the measurement positions located outside the shielding, the count rate of the TEPC detector is so low that it is impractical to collect data with good statistics even at a high proton current and a long counting time. Therefore, for these positions, a portable ion chamber gamma meter (model 9DP, Ludlum Measurement, Inc.) and a portable neutron dose meter (model 12-4, Ludlum Measurement, Inc.) were employed for gamma-ray and neutron dose rate measurements.

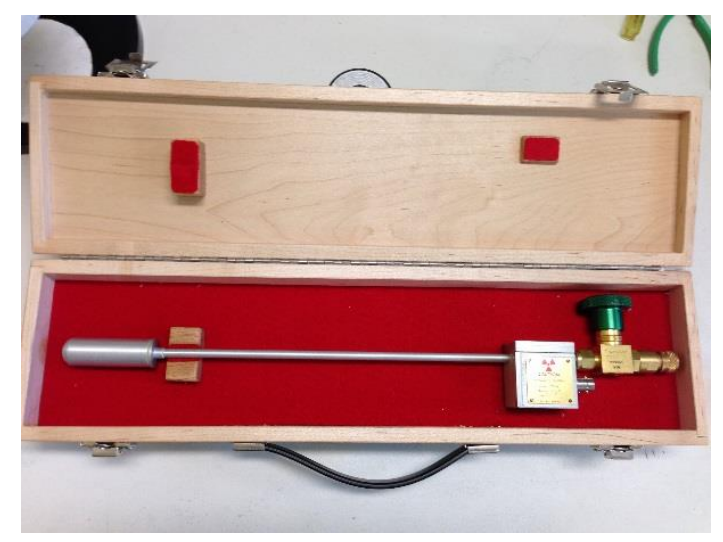

g.

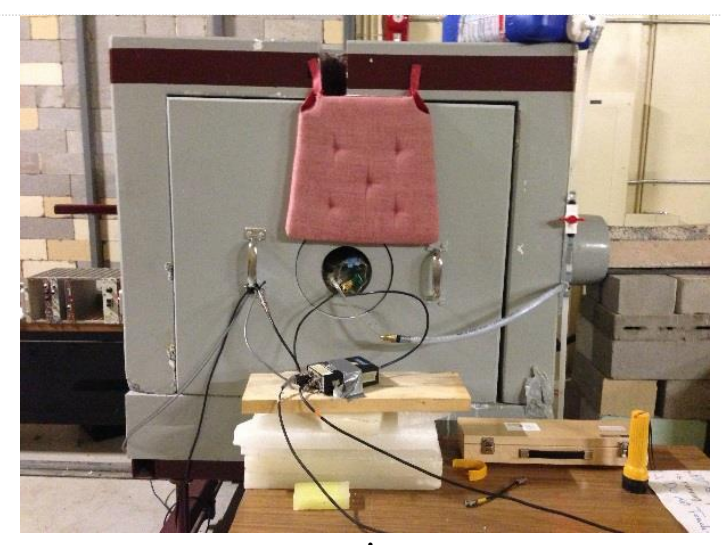

i.

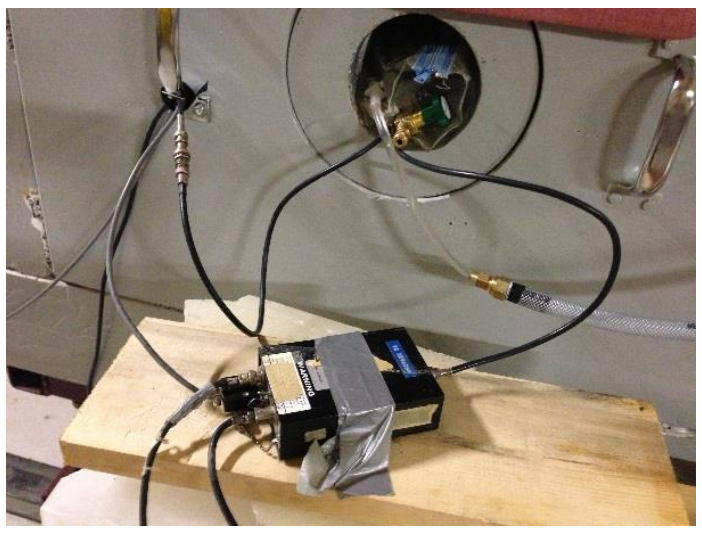

h.

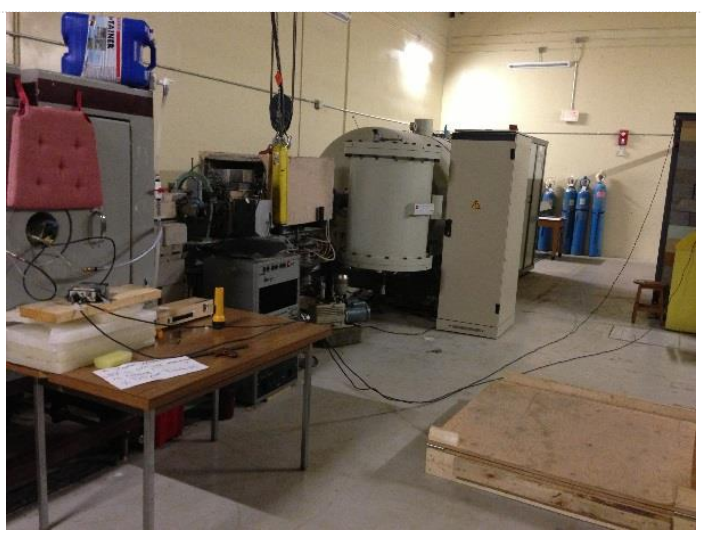

$\mathrm{j}$.

Fig. 6.1 Experimental setup for dosimetry study using commercial TEPC. a. Far West $1 / 2$ ” TEPC, b. The water bag placed around the detector in the arm access hole. Detector is connected to preamp and HV 


\subsection{Results and Discussion}

Data analysis methodology, thoroughly explained in the previous chapter, was employed to analyze the raw data collected. Fig. 6.2 shows the redistributed normalized microdosimetric spectra collected at the center of the hand irradiation position for the various incident proton energies, from 1.8 to $2.5 \mathrm{MeV}$. For convenience, the vertical scale is quoted in terms of $\mathrm{yN}(\mathrm{y})$ normalised by the total proton charge incident on the lithium target. In each spectrum, the neutron and gamma-ray components were separated by a fit using a standard gamma-ray microdosimetric spectrum from ${ }^{137} \mathrm{Cs}$ (Waker et al 1995) energies. The area under the neutron or gamma-ray $\mathrm{yN}(\mathrm{y})$ curve is proportional to the absorbed dose, which was determined by eq. 5-2 and 5-3.

In this figure, at $\mathrm{E}_{\mathrm{p}}=1.8 \mathrm{MeV}$, below the threshold of the ${ }^{7} \mathrm{Li}(\mathrm{p}, \mathrm{n})$ reaction, there is only a gamma-ray component and no neutron component is observable at all. With the increase of the incident proton energy from 1.95 to $2.5 \mathrm{MeV}$, the neutron dose component increases rapidly, about a factor of 80 times, owing to the sensitive dependence of the ${ }^{7} \mathrm{Li}(\mathrm{p}, \mathrm{n})$ neutron yield on proton energy in this region. Compared with the gamma-ray absorbed dose, the neutron absorbed dose is a little lower than the gamma dose at $\mathrm{E}_{\mathrm{p}}=1.95$ $\mathrm{MeV}$, while it is greatly higher than the gamma dose at $\mathrm{E}_{\mathrm{p}}=2.5 \mathrm{MeV}$. It is noted in the figure that the width of the neutron peak is rather narrow for the incident proton energies of 1.95 and $2.0 \mathrm{MeV}$, while it is rather broad for higher proton energies. This is caused by the fact that at the incident proton energies of 1.95 and $2.0 \mathrm{MeV}$, the neutron and corresponding recoil proton energies are relatively low, which make most recoil protons completely stop in the gaseous sensitive volume of the TEPC, while the fraction of the complete stopping events decreases as the recoil proton range increases for higher incident proton energies. 

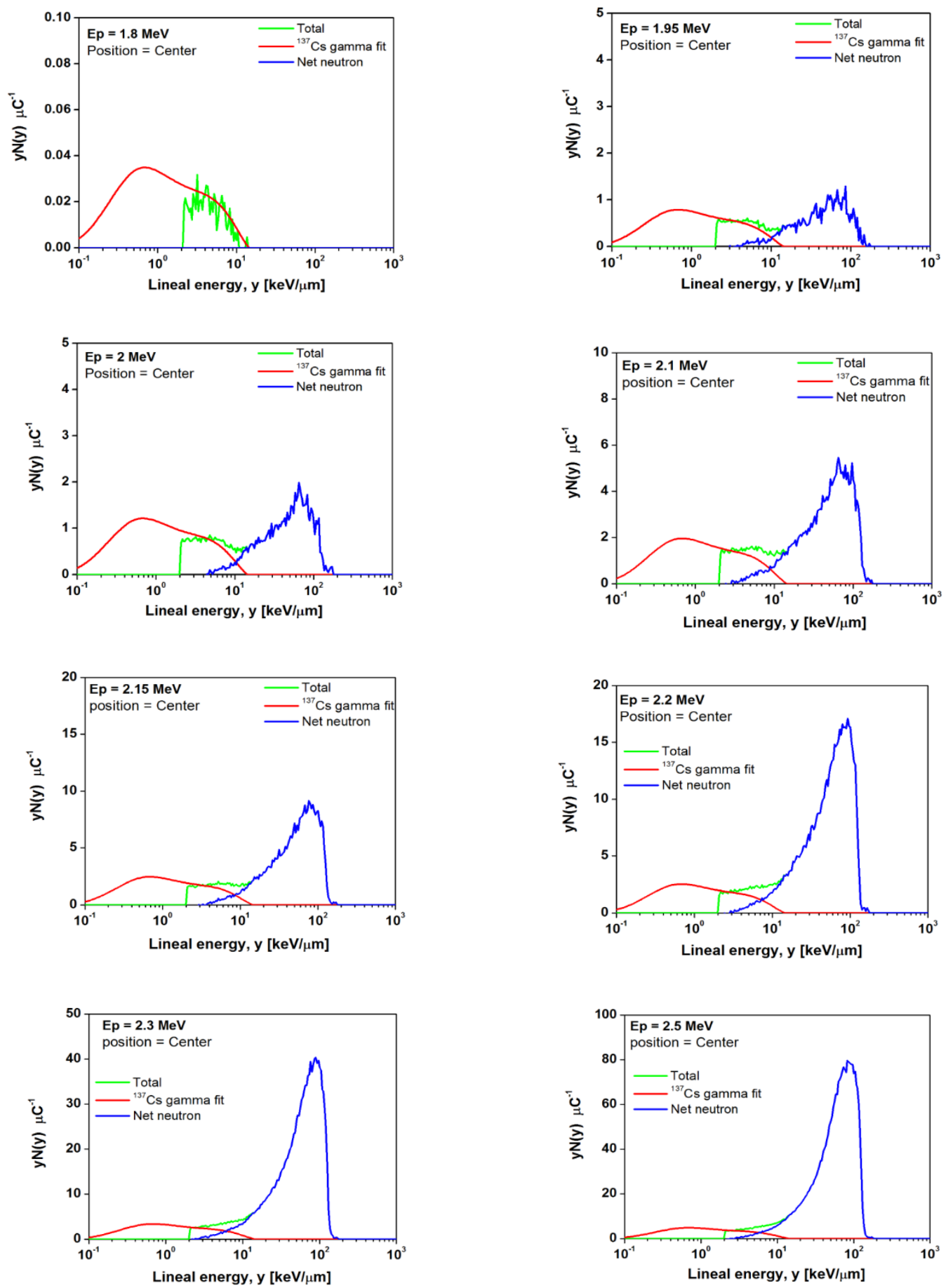

Fig. 6.2 Microdosimetric spectra collected at the center of the irradiation position for different proton energies (Total: the measured spectrum, ${ }^{137} \mathrm{Cs}$ gamma-ray fit: gamma fit using ${ }^{137} \mathrm{Cs}$ pure gamma spectrum, Net neutron: subtraction of gamma fit from the total) 
Fig. 6.3 shows the microdosimetric spectra collected for $\mathrm{E}_{\mathrm{p}}=2.3 \mathrm{MeV}$ at various lateral positions along the arm axis in order to investigate the spatial distributions of the neutron and gamma-ray doses. As shown in the figure, the neutron absorbed dose decreases as the position is shifted away from the hand center up to $20 \mathrm{~cm}$ and then rapidly decreases at farther positions due to the water shielding surrounding the arm. The gamma-ray dose rate decreases much more slowly in contrast to the neutron dose. The spatial neutron and gamma dose distributions for other incident proton energies showed similar trends with Fig. 6.3.

The microdosimetry spectra for proton energies of 1.95, 2, 2.1, 2.15, 2.2, $2.5 \mathrm{MeV}$ at various positions of 0 (center of cavity), 3, 6, 10,15,20,25, $30 \mathrm{~cm}$ are shown in Figs. 6.4 to 6.9 .

As a mixed field, both neutrons and gamma-rays contribute to the absorbed dose. Using the data analysis method explained in chapter 5, the neutron and gamma-ray fractions of absorbed dose rate were calculated for all the energies and positions and represented in table 6-1 and 6-2. Absorbed dose rates from the gamma rays were higher than the neutron fraction for the lower proton energies (1.95 and $2 \mathrm{MeV})$ at all the positions. By increasing the proton energy and for positions closer to the center of the cavity, the fraction contribution of the gamma rays in absorbed dose decreases and neutron absorbed dose fraction contribution dominates. 

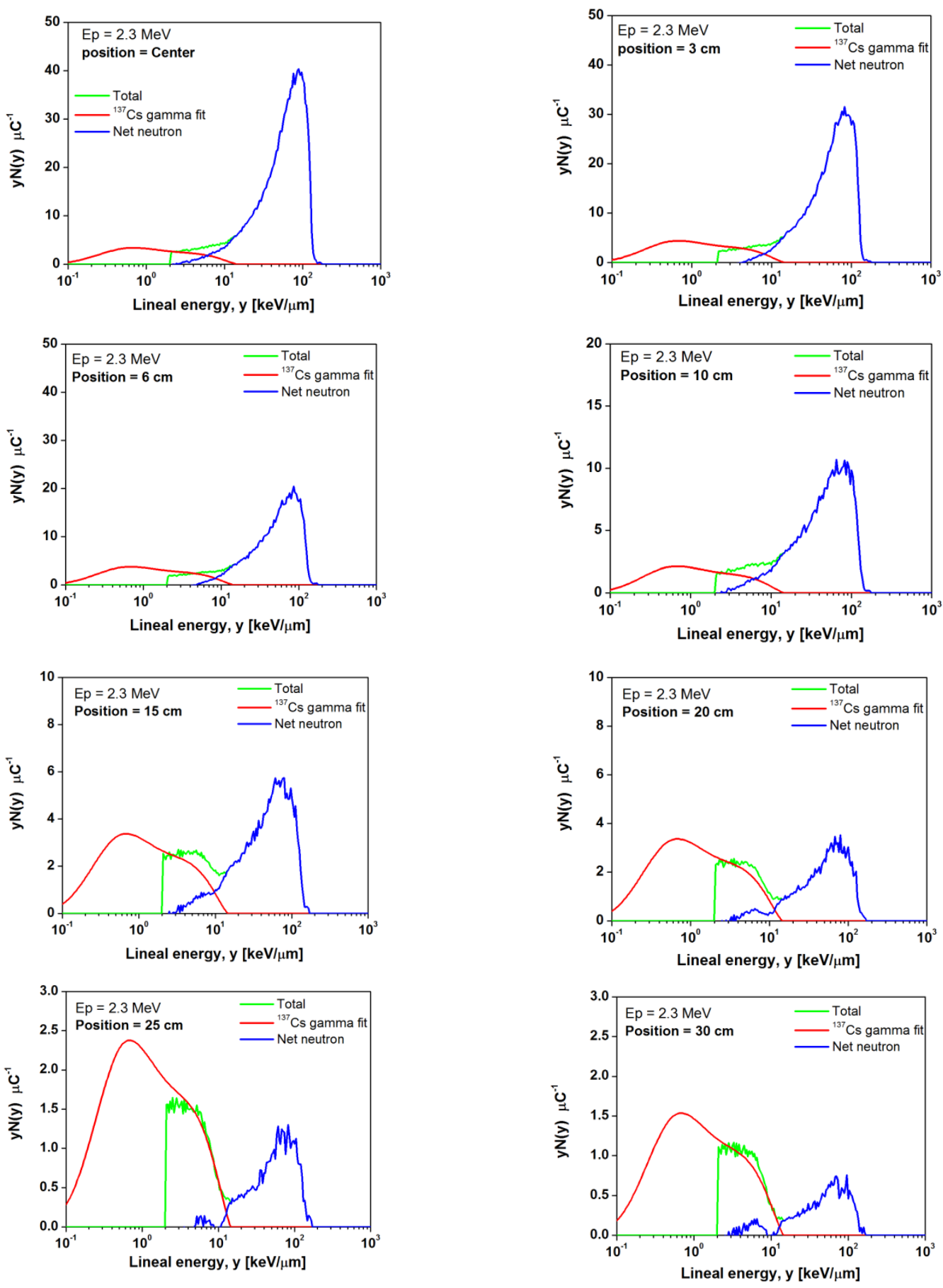

Fig. 6.3 Microdosimetric spectra collected at various lateral positions for $E_{p}=2.3 \mathrm{MeV}$ 

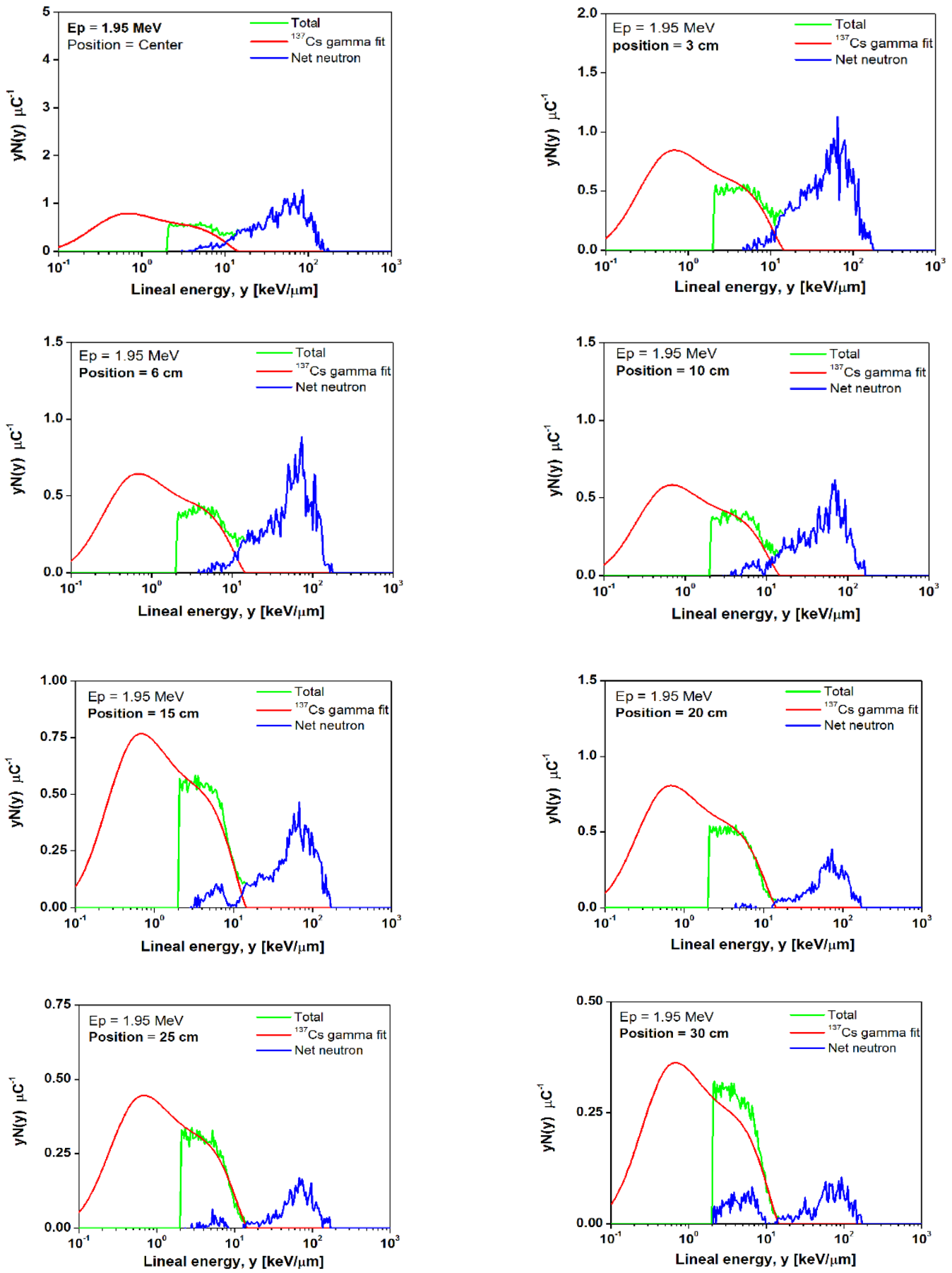

Fig. 6.4 Microdosimetric spectra collected at various lateral positions for $E_{p}=1.95 \mathrm{MeV}$ 

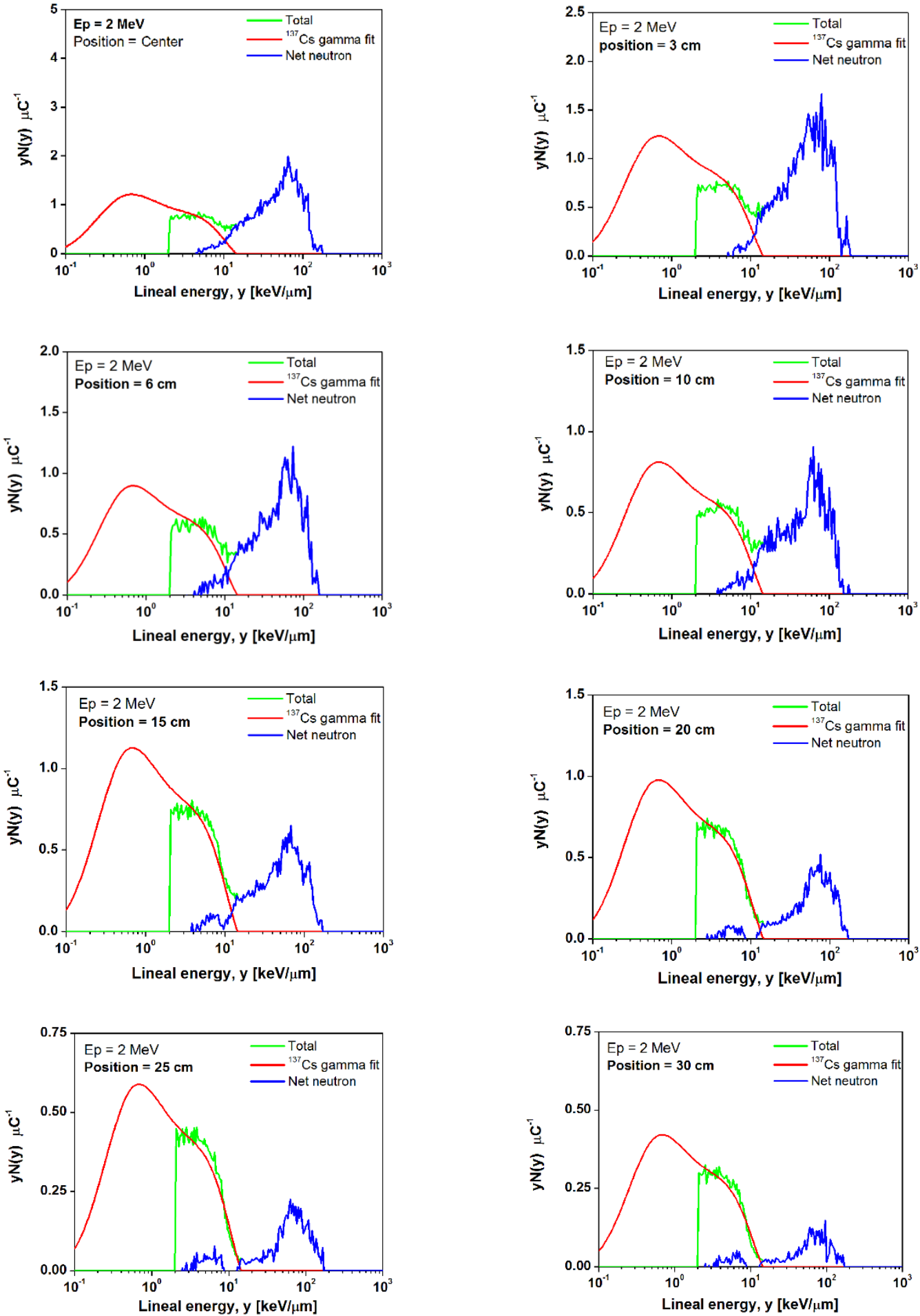

Fig. 6.5 Microdosimetric spectra collected at various lateral positions for $E_{p}=2 \mathrm{MeV}$ 

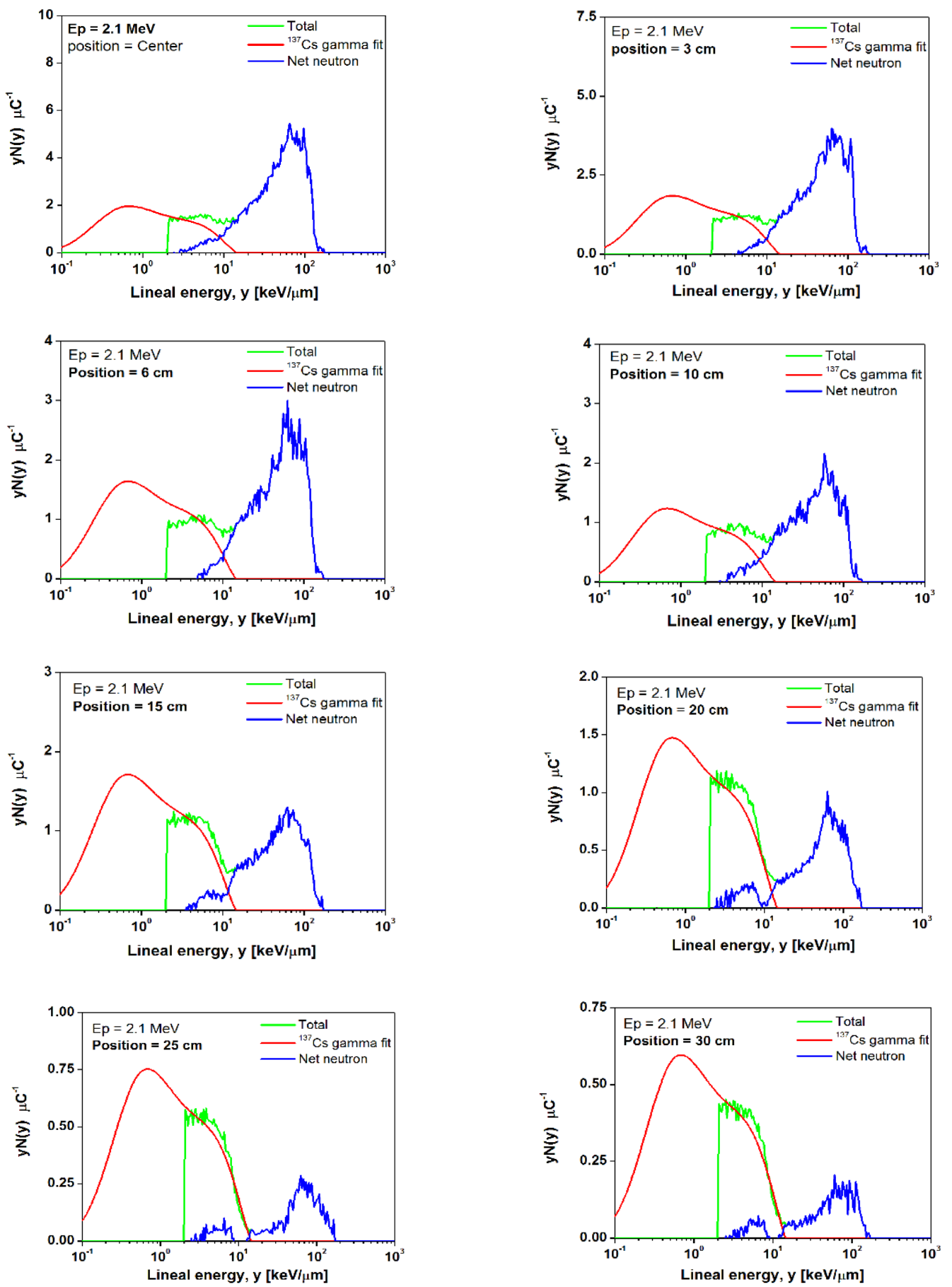

Fig. 6.6 Microdosimetric spectra collected at various lateral positions for $E_{p}=2.1 \mathrm{MeV}$ 

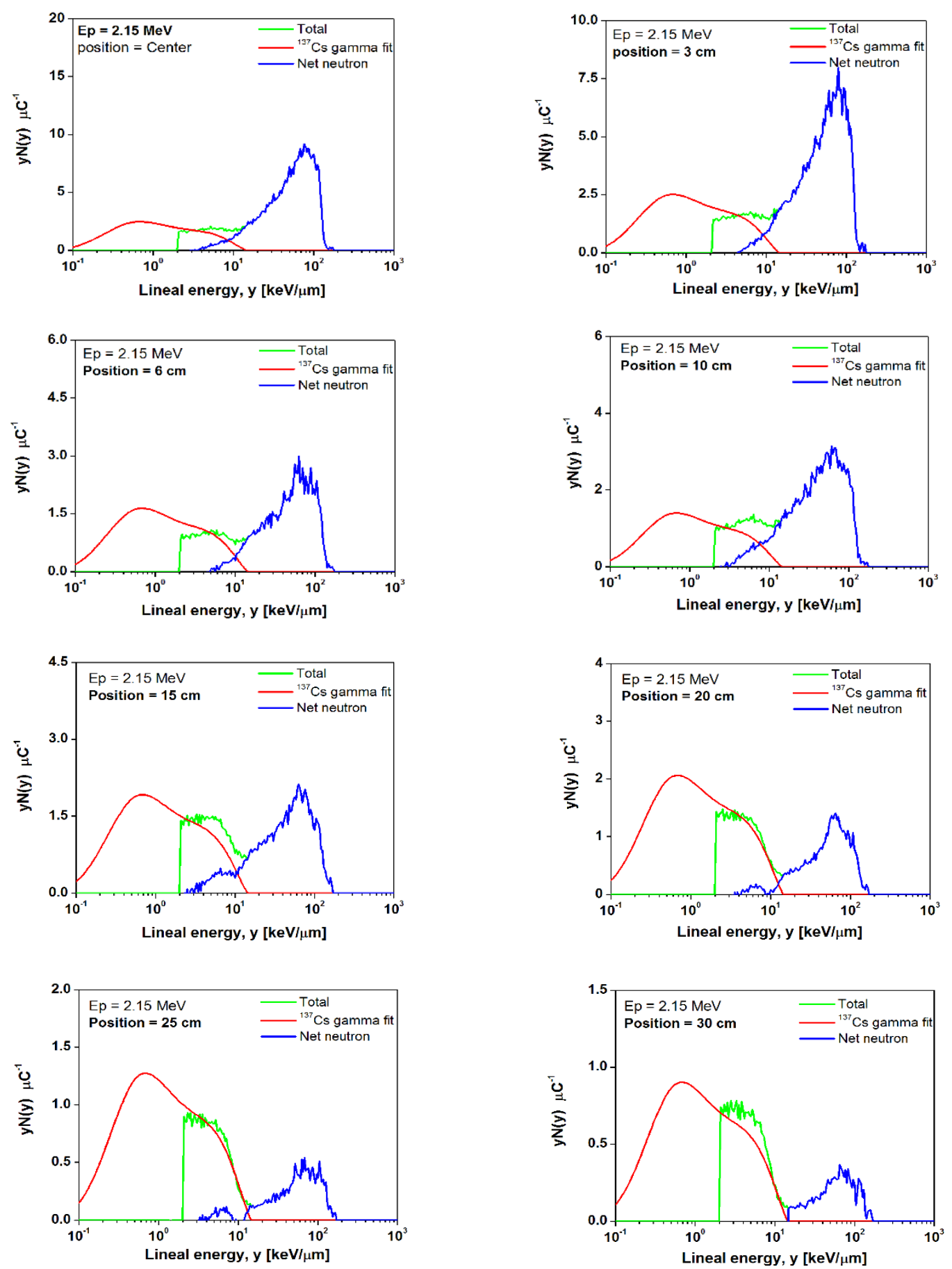

Fig. 6.7 Microdosimetric spectra collected at various lateral positions for $E_{p}=2.15 \mathrm{MeV}$ 

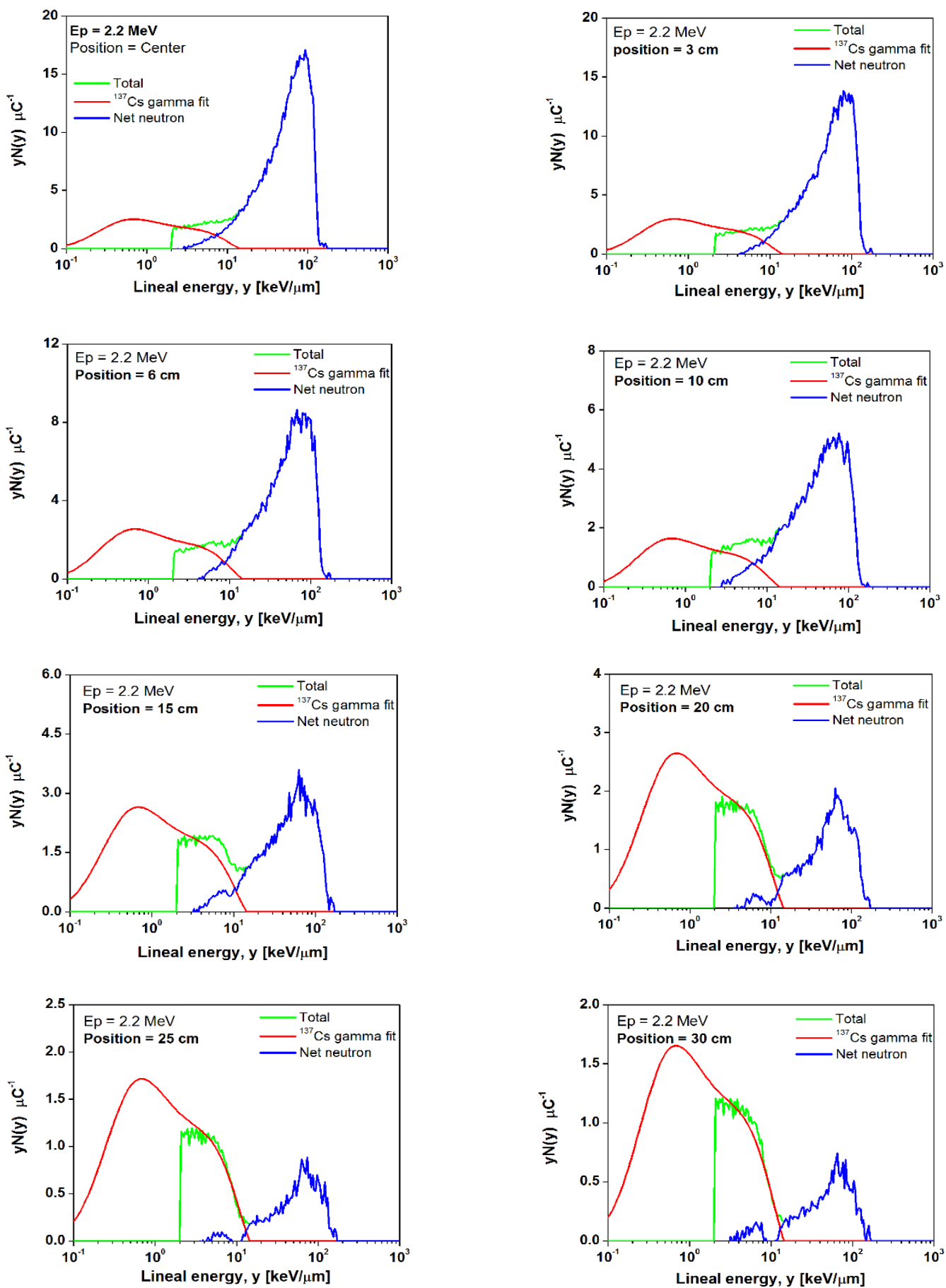

Fig. 6.8 Microdosimetric spectra collected at various lateral positions for $E_{p}=2.2 \mathrm{MeV}$ 

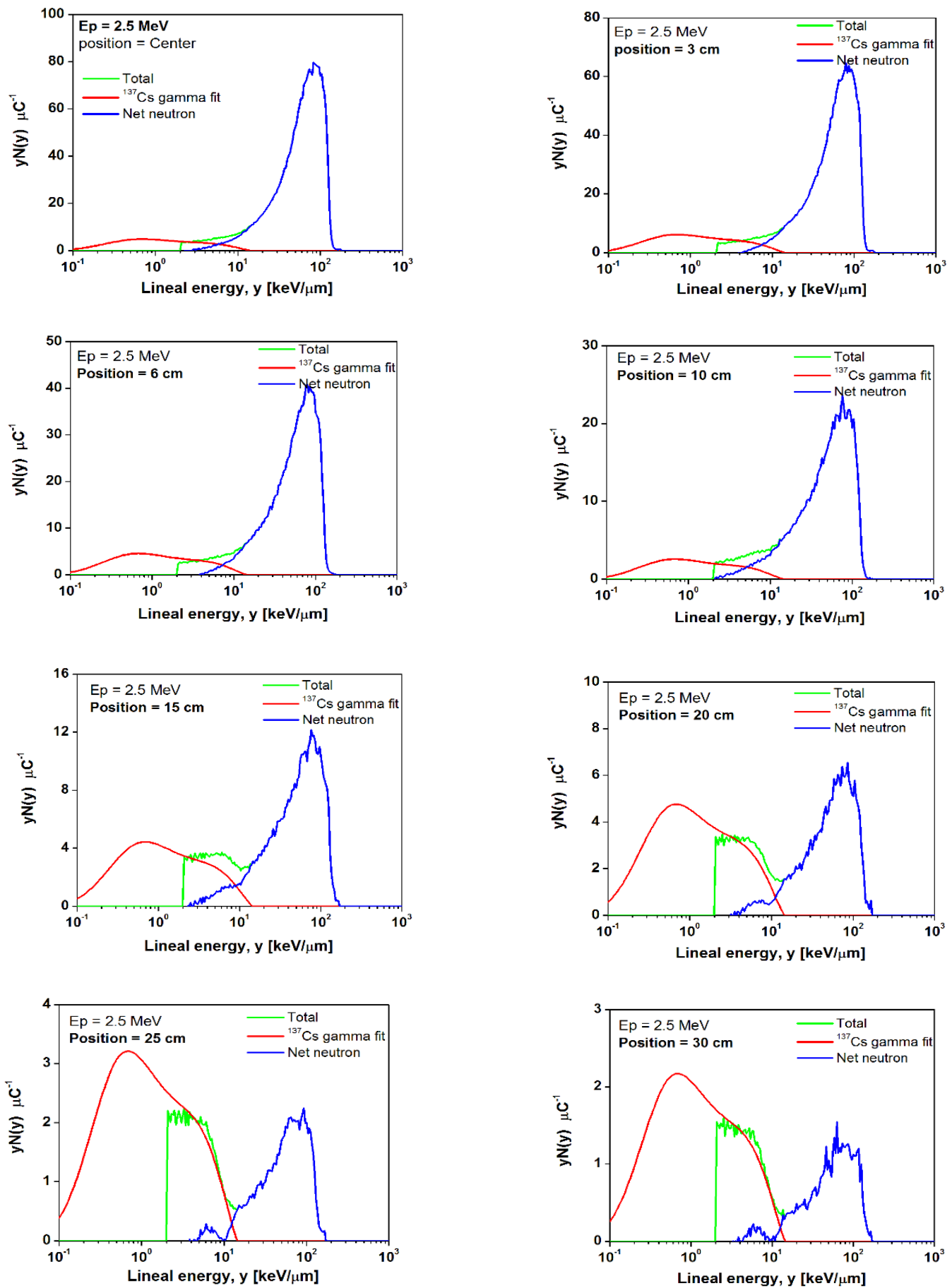

Fig. 6.9 Microdosimetric spectra collected at various lateral positions for $E_{p}=2.5 \mathrm{MeV}$ 
Table 6-1. Neutron absorbed dose values. All the values are in $\mathrm{mGy}_{\mu} \mathrm{A}^{-1} \mathrm{~min}^{-1}$ and $\pm 10 \%$

\begin{tabular}{cccccccc}
\hline \multirow{2}{*}{$\begin{array}{c}\text { Position } \\
(\mathrm{cm})\end{array}$} & $\mathbf{1 . 9 5}$ & $\mathbf{2}$ & $\mathbf{2 . 1}$ & $\mathbf{2 . 1 5}$ & $\mathbf{2 . 2}$ & $\mathbf{2 . 3}$ & $\mathbf{2 . 5}$ \\
\cline { 2 - 8 } & 0.0034 & 0.0053 & 0.0169 & 0.0275 & 0.0482 & 0.1116 & 0.2224 \\
$\mathbf{0}$ & 0.0027 & 0.0043 & 0.0122 & 0.0225 & 0.0407 & 0.0897 & 0.1755 \\
$\mathbf{3}$ & 0.0020 & 0.0032 & 0.0085 & 0.0158 & 0.0271 & 0.0562 & 0.1155 \\
$\mathbf{6}$ & 0.0014 & 0.0024 & 0.0066 & 0.0114 & 0.0189 & 0.0363 & 0.0723 \\
$\mathbf{1 0}$ & 0.0011 & 0.0018 & 0.0043 & 0.0070 & 0.0107 & 0.0191 & 0.0365 \\
$\mathbf{1 5}$ & 0.0007 & 0.0012 & 0.0023 & 0.0037 & 0.0055 & 0.0101 & 0.0188 \\
$\mathbf{2 0}$ & 0.0003 & 0.0005 & 0.0006 & 0.0013 & 0.0020 & 0.0034 & 0.0062 \\
$\mathbf{2 5}$ & 0.0001 & 0.0002 & 0.0005 & 0.0008 & 0.0011 & 0.0019 & 0.0038 \\
$\mathbf{3 0}$ & & & & & & & \\
\hline
\end{tabular}

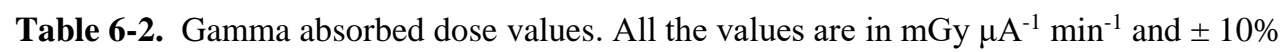

\begin{tabular}{cccccccc}
\hline \multirow{2}{*}{$\begin{array}{c}\text { Position } \\
(\mathrm{cm})\end{array}$} & $\mathbf{1 . 9 5}$ & $\mathbf{2}$ & $\mathbf{2 . 1}$ & $\mathbf{2 . 1 5}$ & $\mathbf{2 . 2}$ & $\mathbf{2 . 3}$ & $\mathbf{2 . 5}$ \\
\cline { 2 - 8 } & 0.0053 & 0.009 & 0.0128 & 0.0162 & 0.0193 & 0.0230 & 0.0339 \\
$\mathbf{0}$ & 0.0053 & 0.0080 & 0.0120 & 0.0138 & 0.0163 & 0.0204 & 0.0294 \\
$\mathbf{3}$ & 0.0041 & 0.0057 & 0.0106 & 0.0115 & 0.0133 & 0.0178 & 0.0239 \\
$\mathbf{6}$ & 0.0033 & 0.0048 & 0.0084 & 0.0093 & 0.0110 & 0.0143 & 0.0171 \\
$\mathbf{1 0}$ & 0.0054 & 0.0071 & 0.0107 & 0.0120 & 0.0166 & 0.0211 & 0.0278 \\
$\mathbf{1 5}$ & 0.0051 & 0.0061 & 0.0099 & 0.0129 & 0.0166 & 0.0211 & 0.0298 \\
$\mathbf{2 0}$ & 0.0025 & 0.0034 & 0.0047 & 0.0080 & 0.0108 & 0.0149 & 0.0201 \\
$\mathbf{2 5}$ & 0.0016 & 0.0026 & 0.0043 & 0.0057 & 0.0071 & 0.0096 & 0.0136 \\
$\mathbf{3 0}$ & & & & & & & \\
\hline
\end{tabular}

To determine the equivalent dose at each position for a given proton energy, the corresponding neutron weighting factor was required. To this end, MCNP5 Monte Carlo simulations were carried out by Dr. Soo Hyun Byun (Darvish-Molla et al, 2015). For a given incident proton energy, the corresponding neutron energy and angular distributions were coded into the source card and neutron fluence spectra were tallied at the positions of interests. The neutron energy groups of interests are thermal $(\mathrm{En}<0.5 \mathrm{eV})$, epithermal $(0.5$ $\mathrm{eV}<\mathrm{En}<5 \mathrm{keV}$ ) and 80 fast groups with $10 \mathrm{keV}$ intervals. 
Using the simulation data the neutron fluence spectra were computed at various positions. Fig. 6.10 shows the simulated neutron fluence spectra at the hand irradiation positions (center and $6 \mathrm{~cm}$ shifted with respect to the $\mathrm{Y}$ axis in the direction towards the arm access hole) for different incident proton energies. For convenience, the fluence spectrum is quoted in terms of neutron fluence per unit lethargy per unit proton charge $\left(\mathrm{cm}^{-2} \mathrm{mC}^{-1}\right)$ so that the area under a curve for a given logarithmic energy interval represents the neutron fluence in that energy interval.

As the incident proton energy increases, neutron fluence increases rapidly due to the increase in neutron yield and the maximum neutron energy is extended from the $(p, n)$ reaction kinematics (Lee and Zhou 1999, Matysiak et al 2008). The fast neutron fluence is relatively weak for lower proton energies, and its relative fraction keeps increasing with the increase in the incident proton energy. When the position is laterally shifted by $6 \mathrm{~cm}$ from the center position, both fast and epithermal fluences decrease due to the distance effect, while the thermal fluence does not show a noticeable change, which indicates a relatively uniform field for thermal neutrons.

Fig. 6.11 shows the spatial distribution of the neutron spectrum along the lateral axis for $E_{p}=2.0$ and $2.3 \mathrm{MeV}$. For both proton energies, the fast neutron fluence rapidly dies out as the position is shifted farther due to the scattering and moderation effect in the graphite reflector and water bag, while the thermal neutron fluence decreases much less. At the $30 \mathrm{~cm}$ lateral position, the thermal, epithermal and fast fluences are reduced by 96 , 99.9 and $99.99 \%$, respectively, in contrast to the center position for both $\mathrm{E}_{\mathrm{p}}=2.0$ and $2.3 \mathrm{MeV}$. 


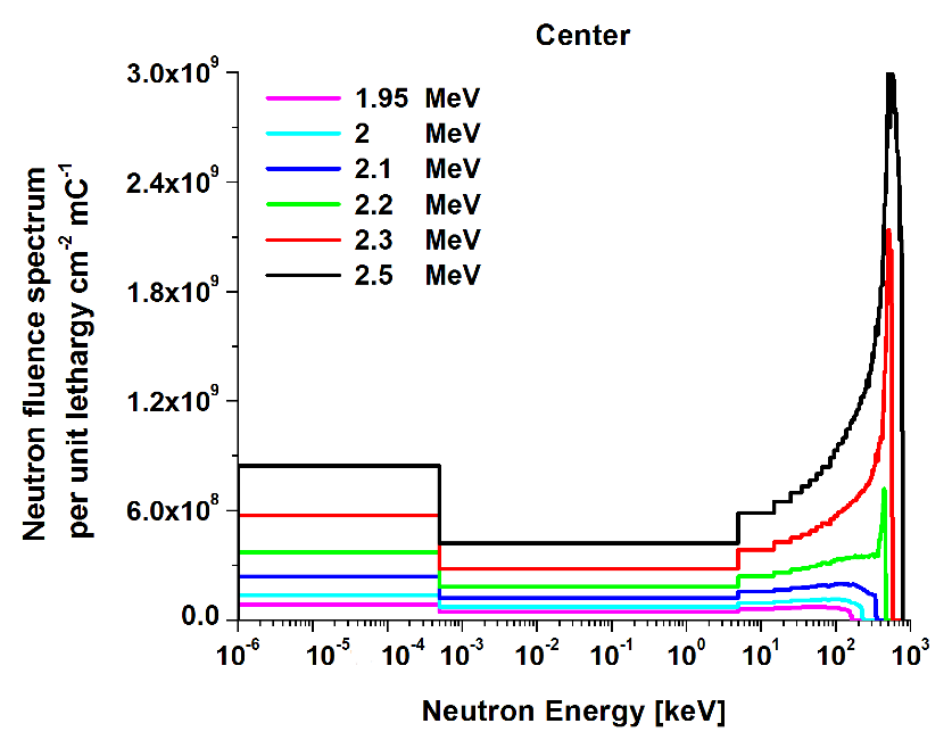

a.

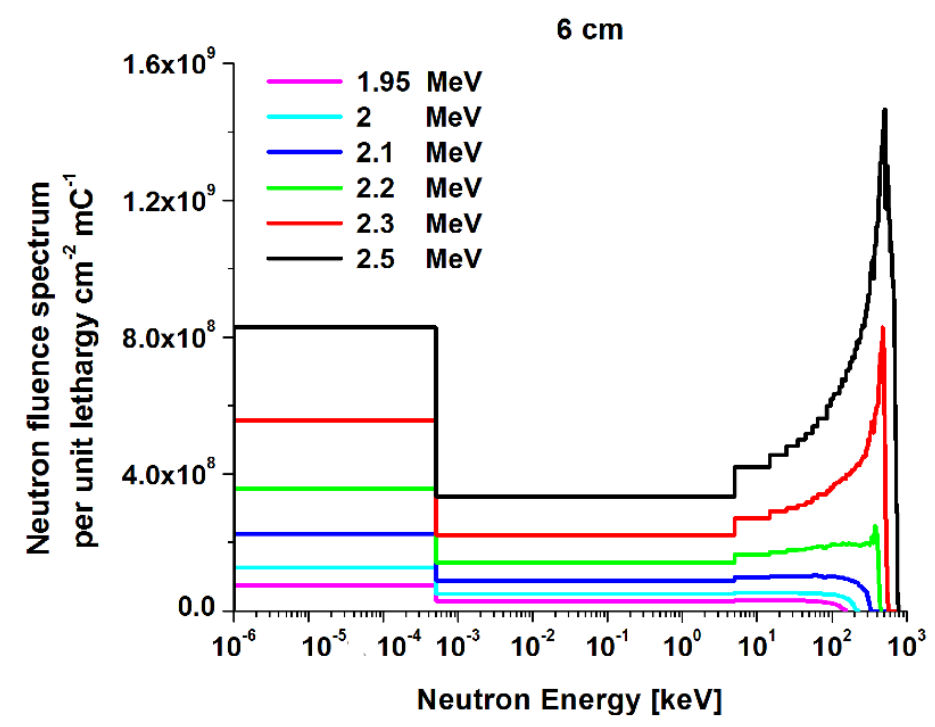

b.

Fig. 6.10 Neutron fluence spectra per unit lethargy per unit proton charge simulated at two different positions: (a) center of the cavity and (b) $6 \mathrm{~cm}$ shifted towards arm access for various incident proton energies. (Darvish-Molla et al, 2015) 


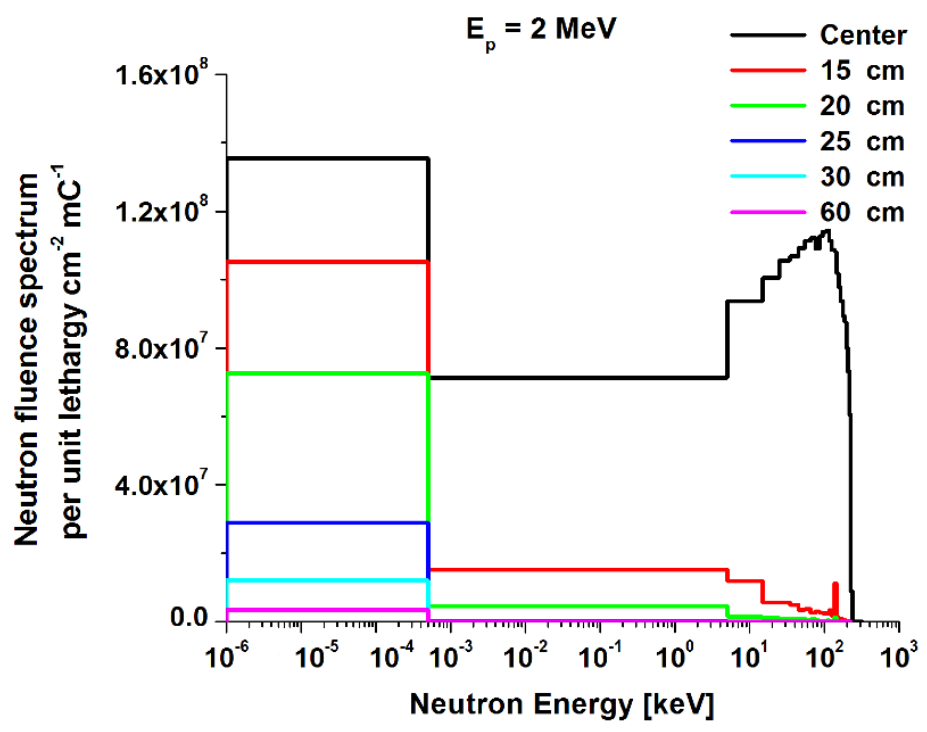

a.

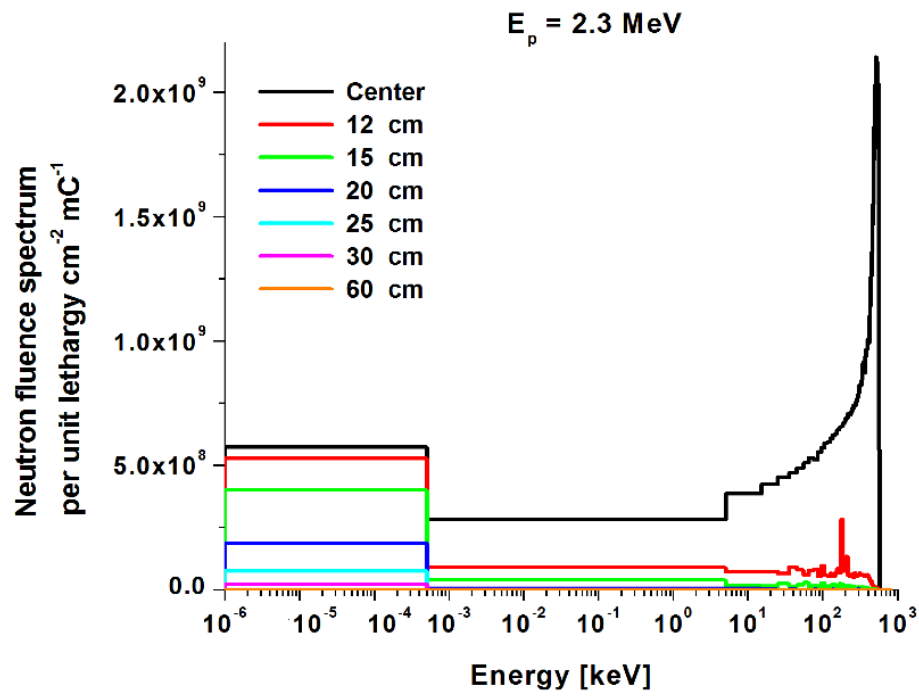

b.

Fig. 6.11 Neutron fluence spectra per unit lethargy per unit proton charge simulated at various lateral positions along the arm for: (a) $E_{p}=2 \mathrm{MeV}$ and (b) $E_{p}=2.3 \mathrm{MeV}$. (Darvish-Molla et al, 2015) 
Using the neutron fluence spectrum data and the recommended ICRP 60 formula for weighting factor (ICRP 1991), the neutron weighting factor at each position for a given proton energy was computed and the results are shown in Fig. 6.12. As shown in the figure, the neutron weighting factor is maximum at the center position since the relative contribution of fast neutrons is maximum at this position. The neutron weighting factors at the center vary from 6.1 to 9.4 depending on the proton energy. As the position is shifted, the neutron weighting factor keeps decreasing up to $20 \mathrm{~cm}$ and then saturates to 5 at farther positions, which stems from the fact that the fast neutron contribution is negligible beyond $20 \mathrm{~cm}$.

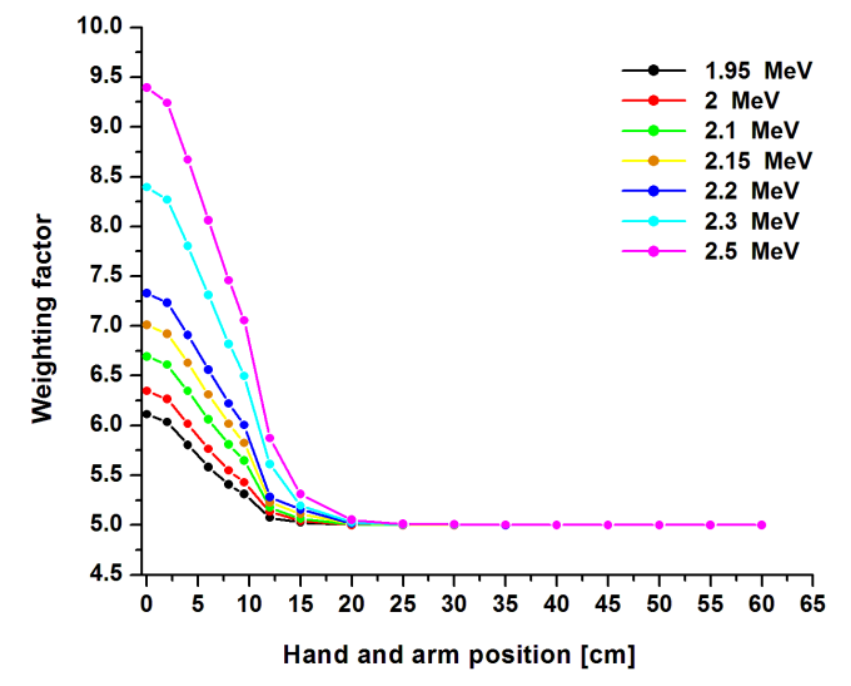

Fig. 6.12 Neutron weighting factor as a function of the lateral position for all proton energies (Darvish-Molla et al, 2015) 
Ultimately, having absorbed doses and corresponding weighting factors information, the neutron and gamma-ray equivalent doses for each proton energy and position were calculated as listed in tables 6-3 and 6-4. At the center position, the neutron equivalent dose increases about 100 times with the increase of the proton energy from 1.95 to $2.5 \mathrm{MeV}$, due to the rapid increases in neutron yield and the weighting factor. As the position is shifted $30 \mathrm{~cm}$ away from the center, the neutron weighting factor is less dependent on the incident proton energy as observed in Fig. 6.12, resulting in a factor of about 30 difference between neutron equivalent doses for $\mathrm{E}_{\mathrm{p}}=1.95$ and $2.5 \mathrm{MeV}$. On the other hand, the gamma-ray equivalent dose is less dependent on the proton energy and position.

Table 6-3. Neutron equivalent dose values. All the values are in $\mathrm{mSv}_{\mu} \mathrm{A}^{-1} \mathrm{~min}^{-1} \pm 10 \%$

\begin{tabular}{cccccccc}
\hline \multirow{2}{*}{$\begin{array}{c}\text { Position } \\
(\mathrm{cm})\end{array}$} & $\mathbf{1 . 9 5}$ & $\mathbf{2}$ & $\mathbf{2 . 1}$ & $\mathbf{2 . 1 5}$ & $\mathbf{2 . 2}$ & $\mathbf{2 . 3}$ & $\mathbf{2 . 5}$ \\
\cline { 2 - 7 } & 0.0208 & 0.0334 & 0.1128 & 0.1931 & 0.3535 & 0.9363 & 2.0898 \\
$\mathbf{0}$ & 0.0161 & 0.0285 & 0.0788 & 0.1525 & 0.2876 & 0.7207 & 1.5721 \\
$\mathbf{3}$ & 0.0111 & 0.0185 & 0.0516 & 0.1000 & 0.1776 & 0.4108 & 0.9312 \\
$\mathbf{6}$ & 0.0075 & 0.0127 & 0.0361 & 0.0638 & 0.1087 & 0.2258 & 0.4821 \\
$\mathbf{1 0}$ & 0.0057 & 0.0090 & 0.0216 & 0.0359 & 0.0553 & 0.0990 & 0.1938 \\
$\mathbf{1 5}$ & 0.0035 & 0.0059 & 0.0116 & 0.0187 & 0.0276 & 0.0508 & 0.0949 \\
$\mathbf{2 0}$ & 0.0015 & 0.0023 & 0.0029 & 0.0065 & 0.0101 & 0.0170 & 0.0312 \\
$\mathbf{2 5}$ & 0.007 & 0.0012 & 0.0067 & 0.0038 & 0.0056 & 0.0097 & 0.0190 \\
$\mathbf{3 0}$ & & & & & &
\end{tabular}

Table 6-4. Gamma equivalent dose values. All the values are in $\mathrm{mSv}_{\mu} \mathrm{A}^{-1} \mathrm{~min}^{-1} \pm 10 \%$

\begin{tabular}{cccccccc}
\hline \multirow{2}{*}{$\begin{array}{c}\text { Position } \\
(\mathrm{cm})\end{array}$} & $\mathbf{1 . 9 5}$ & $\mathbf{2}$ & $\mathbf{2 . 1}$ & $\mathbf{2 . 1 5}$ & $\mathbf{2 . 2}$ & $\mathbf{2 . 3}$ & $\mathbf{2 . 5}$ \\
\cline { 2 - 8 } & 0.0053 & 0.009 & 0.0128 & 0.0162 & 0.0193 & 0.0230 & 0.0339 \\
$\mathbf{0}$ & 0.0053 & 0.0080 & 0.0120 & 0.0138 & 0.0163 & 0.0204 & 0.0294 \\
$\mathbf{3}$ & 0.0041 & 0.0057 & 0.0106 & 0.0115 & 0.0133 & 0.0178 & 0.0239 \\
$\mathbf{6}$ & 0.0033 & 0.0048 & 0.0084 & 0.0093 & 0.0110 & 0.0143 & 0.0171 \\
$\mathbf{1 0}$ & 0.0054 & 0.0071 & 0.0107 & 0.0120 & 0.0166 & 0.0211 & 0.0278 \\
$\mathbf{1 5}$ & 0.0051 & 0.0061 & 0.0099 & 0.0129 & 0.0166 & 0.0211 & 0.0298 \\
$\mathbf{2 0}$ & 0.0025 & 0.0034 & 0.0047 & 0.0080 & 0.0108 & 0.0149 & 0.0201 \\
$\mathbf{2 5}$ & 0.0016 & 0.0026 & 0.0043 & 0.0057 & 0.0071 & 0.0096 & 0.0136 \\
$\mathbf{3 0}$ & & & & & & &
\end{tabular}


Total equivalent dose values for each proton energy and position are shown in Table 6-5 and Fig. 6.13. Since the radiation weighting factors for neutrons are 5-10 times higher than for gamma rays as observed in Fig. 6.12, neutrons contribute dominantly to the total equivalent dose for most positions except for those positions beyond $20 \mathrm{~cm}$ and lower proton energies.

A $10 \%$ uncertainty, which is generally accepted for dose values obtained from microdosimetric spectra, is a conservative estimation that takes into account the uncertainties due to the filling gas pressure, internal alpha source positioning, lineal energy calibration, etc. (ICRU 1983; Spirou et al, 2008).

Table 6-5. Total equivalent dose values. All the values are in $\mathrm{mSv}_{\mu} \mathrm{A}^{-1} \min ^{-1} \pm 10 \%$

\begin{tabular}{cccccccc}
\hline \multirow{2}{*}{$\begin{array}{c}\text { Position } \\
(\mathrm{cm})\end{array}$} & $\mathbf{1 . 9 5}$ & $\mathbf{2}$ & $\mathbf{2 . 1}$ & $\mathbf{2 . 1 5}$ & $\mathbf{2 . 2}$ & $\mathbf{2 . 3}$ & $\mathbf{2 . 5}$ \\
\cline { 2 - 8 } & 0.0262 & 0.0425 & 0.1256 & 0.2093 & 0.3728 & 0.9594 & 2.1237 \\
$\mathbf{0}$ & 0.0214 & 0.0365 & 0.0908 & 0.1663 & 0.3039 & 0.7411 & 1.6015 \\
$\mathbf{3}$ & 0.0152 & 0.0242 & 0.0622 & 0.1115 & 0.1908 & 0.4287 & 0.9551 \\
$\mathbf{6}$ & 0.0109 & 0.0175 & 0.0444 & 0.0732 & 0.1196 & 0.2401 & 0.4992 \\
$\mathbf{1 0}$ & 0.0111 & 0.0161 & 0.0324 & 0.0480 & 0.0719 & 0.1202 & 0.2216 \\
$\mathbf{1 5}$ & 0.0086 & 0.0120 & 0.0216 & 0.0316 & 0.0442 & 0.0719 & 0.1247 \\
$\mathbf{2 0}$ & 0.0040 & 0.0056 & 0.0076 & 0.0145 & 0.0209 & 0.0319 & 0.0513 \\
$\mathbf{2 5}$ & 0.0024 & 0.0039 & 0.0109 & 0.0095 & 0.0127 & 0.0193 & 0.0326 \\
$\mathbf{3 0}$ & & & & & & &
\end{tabular}

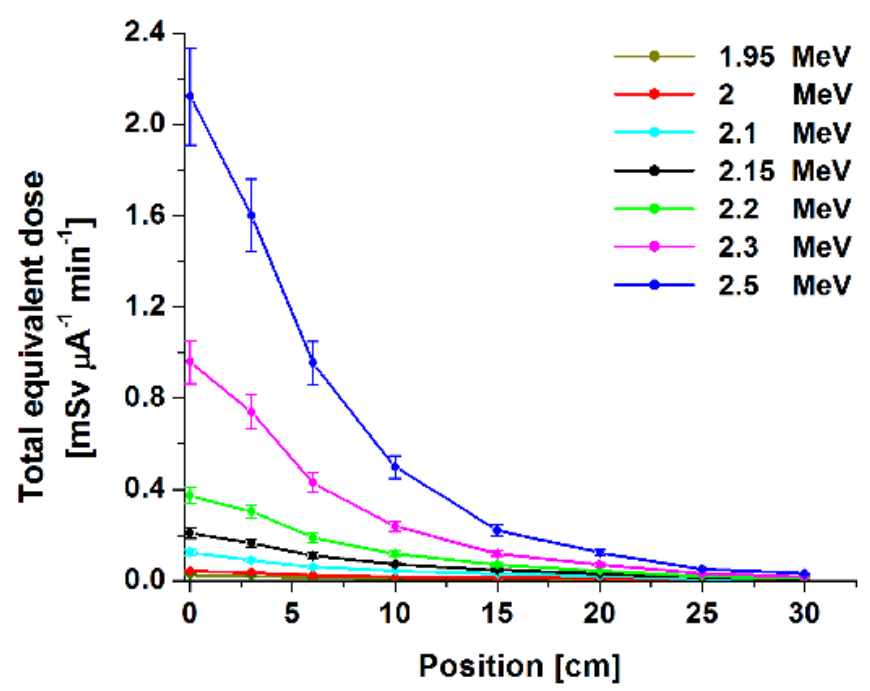

Fig. 6.13 Spatial distribution of total equivalent dose for different proton energies (from 1.9 to $2.5 \mathrm{MeV}$ ) (Darvish-Molla et al, 2015) 
In order to find the neutron and gamma-ray doses for the rest of the body, doses were measured using portable neutron and gamma dose survey meters on the outermost surface of the shield wall, on the $\mathrm{XZ}$ plane, at the center of the arm access hole, $15 \mathrm{~cm}$ above and $25 \mathrm{~cm}$ below. Doses were also measured on the $\mathrm{Y}$ axis, $30 \mathrm{~cm}$ away from the outermost shield wall (see tables 6-6 and 6-7). The neutron dose was maximum at the $(X, Z)=(0,0)$ position, while the gamma-ray dose was maximum at the $(X, Z)=(0,15)$ position with a slightly lower value at the $(X, Z)=(0,0)$ position. The maximum neutron dose ranged from 0.0021 to $0.0319 \mathrm{mSv}_{\mu} \mathrm{A}^{-1} \mathrm{~min}^{-1}$, while the maximum gamma-ray dose ranged from 0.0316 to $0.1862 \mathrm{mSv}_{\mu \mathrm{A}} \mathrm{A}^{-1} \mathrm{~min}^{-1}$ for the incident proton energies of $1.95-2.5 \mathrm{MeV}$. The data indicate that for the measurement positions located outside the shielding, the neutron fraction in total dose is very small (for example, only $15 \%$ for $\mathrm{E}_{\mathrm{p}}=2.5 \mathrm{MeV}$ ) as fast neutrons were mostly moderated and absorbed by the shielding material.

Table 6-6. Neutron equivalent dose values for outside. All the values are in $\mathrm{mSv}_{\mu \mathrm{A}}^{-1} \mathrm{~min}^{-1} \pm 10 \%$

\begin{tabular}{cccccccc}
\hline $\begin{array}{c}\text { Position } \\
(\mathrm{cm})\end{array}$ & $\mathbf{1 . 9 5}$ & $\mathbf{2}$ & $\mathbf{2 . 1}$ & $\mathbf{2 . 1 5}$ & $\mathbf{2 . 2}$ & $\mathbf{2 . 3}$ & $\mathbf{2 . 5}$ \\
\cline { 2 - 8 } & 0.0008 & 0.0011 & 0.0016 & 0.0024 & 0.0043 & 0.0048 & 0.0105 \\
$\mathbf{( 0 , 0 , 1 5 )}$ & 0.0021 & 0.0030 & 0.0054 & 0.0061 & 0.0124 & 0.0206 & 0.0319 \\
$\mathbf{( 0 , 0 , 0 )}$ & 0.0001 & 0.0002 & 0.0003 & 0.0007 & 0.0012 & 0.0014 & 0.0034 \\
$\mathbf{( 0 , 0 , - 2 5 )}$ & 0.0014 & 0.0015 & 0.0037 & 0.0044 & 0.0061 & 0.0100 & 0.0181 \\
$\mathbf{( 0 , 3 0 , 0 )}$ & & & & & & & \\
\hline
\end{tabular}

Table 6-7. Gamma equivalent dose values for outside. All the values are in $\mathrm{mSv}_{\mu \mathrm{A}}^{-1} \min ^{-1} \pm 10 \%$

\begin{tabular}{cccccccc}
\hline \multirow{2}{*}{$\begin{array}{c}\text { Position } \\
(\mathrm{cm})\end{array}$} & $\mathbf{1 . 9 5}$ & $\mathbf{2}$ & $\mathbf{2 . 1}$ & $\mathbf{2 . 1 5}$ & $\mathbf{2 . 2}$ & $\mathbf{2 . 3}$ & $\mathbf{2 . 5}$ \\
\cline { 2 - 8 } & 0.0316 & 0.0399 & 0.0658 & 0.0858 & 0.1038 & 0.1370 & 0.1862 \\
$\mathbf{( 0 , 0 , 1 5 )}$ & 0.0287 & 0.0343 & 0.0547 & 0.0670 & 0.0842 & 0.1137 & 0.1520 \\
$\mathbf{( 0 , 0 , 0 )}$ & 0.0100 & 0.0134 & 0.0248 & 0.0308 & 0.0386 & 0.0549 & 0.0694 \\
$\mathbf{( 0 , 0 , - 2 5 )}$ & 0.0184 & 0.0251 & 0.0397 & 0.0492 & 0.0619 & 0.0778 & 0.1074 \\
$\mathbf{( 0 , 3 0 , 0 )}$ & & & & & & & \\
\hline
\end{tabular}




\subsection{Conclusion}

A comprehensive dosimetric characterization for the McMaster Tandetron ${ }^{7} \operatorname{Li}(\mathrm{p}, \mathrm{n})$ neutron facility was completed. From microdosimetric measurements, neutron and gammaray absorbed doses were determined for various positions in the proton energy range of 1.95-2.50 MeV. The neutron absorbed dose showed steep spatial distributions for all proton energies so that neutrons contributed dominantly to the equivalent dose at the irradiation center position, while their contribution was almost negligible in the region outside the shielding. With the increase of proton energy from 1.95 to $2.50 \mathrm{MeV}$, the total equivalent dose at the irradiation position increased by a factor of 80 times mostly due to the rapid increase in neutron yield, while its increase in the region outside the shield was about 6 times with a tolerable maximum value.

Based on comprehensive dosimetry data accomplished in this study, the mixed neutron and gamma-ray field of the McMaster Tandetron accelerator was investigated and characterized thoroughly. The results obtained gave insight on the proton energy dependence and the spatial distribution of neutron and gamma-ray absorbed doses as well as the neutron weighting factor for the Tandetron Accelerator. In addition, ${ }^{7} \mathrm{Li}(\mathrm{p}, \mathrm{n})$ neutron users would be able to determine the proton energy and current to meet their radiation dose limit. 


\section{Chapter 7}

\section{Results and Discussions}

7.1 Single Gaseous Cavity Detector

7.1.1 Signal Performance and Multiplication Gain

7.1.2 Stability and Reproducibility

7.1.3 Microdosimetric Spectrum

7.1.4 Chemically Cleaned THGEM Data

7.1.5 Performance Test Results using prototype MSI-DSP vs. Commercial DSP

7.1.6 2k Data vs. 16k Data

7.1.7 Summary

7.2 2D Multi-volume Detector $(3 \times 3$ gaseous Cavity Array)

7.2.1 2D Single-Layer THGEM Detector Performance

7.2.2 2D Double-Layer THGEM Detector Performance

7.3 Absorbed Dose Distribution

7.4 2D THGEM Detector with Smaller Size Sensitive Volume 


\section{Results and Discussion}

To demonstrate the 2D THEGM detector capabilities, several studies were performed. The outline of the results is as follow:

I) Section 7.1: In order to reduce the unknown factors and to ensure that the detection chain works properly, first the single gaseous cavity detector was tested as explained in the experimental methodology chapter (section 4.2.1). The goal of this investigation was 1) to ascertain the operating voltage of the detector, 2) to obtain a signal successfully, 3) to check the detector stability and reproducibility, 4) to explore the multiplication gain of a single layer THGEM and 5) To compare the performance of the old and new THGEM. Also, the preliminary test results using the prototype MSI-DSP, as explained in 4.2.2, will be presented.

II) Section 7.2: In this section, the 2D multi-volume detector performance was extensively investigated through the outline below:

\section{Single layer THGEM:}

1) First measurement with DSPEC to test if the cables and electronics are working

2) Show the results for the consistency of the MMI-DSP

3) Examine the effect of rotating the detector

4) Show the consistency of the results for $2 \mathrm{MeV}$ and $2.3 \mathrm{MeV}$ protons and different currents

a. $\quad 2.3 \mathrm{MeV}$ and $50 \mu \mathrm{A} \rightarrow$ THGEM \#9, \#10, \#11

b. THGEM \#10 and $50 \mu \mathrm{A} \rightarrow 2,2.3,2.5 \mathrm{MeV}$

c. THGEM \#10 and $2.3 \mathrm{MeV} \rightarrow 50$ and $150 \mu \mathrm{A}$

d. Count rate comparison for 50 and $150 \mu \mathrm{A}$

e. Count rate comparison for $2,2.3$ and $2.5 \mathrm{MeV}$

5) Different gap sizes and type assessment

a. Compare $1 \mathrm{~mm}$ gap and $2 \mathrm{~mm}$ gap result for a center detector

b. Compare $1 \mathrm{~mm}$ gap square and spacer to check if the flatness of layers is important at this stage 


\section{Double layer THGEM:}

1) Compare the gain and discuss the advantage of double layers THGEM

2) Compare the spectra results for the single and double THGEMs

3) Detector performance after 3 months

4) BKG check and the effects

III) Section 7.3: The aim of this section is to compare the absorbed dose rate at the position of the center detector (D5) measured with 1/2" TEPC and Single-THGEM D5 for $2.3 \mathrm{MeV}$. Also to show the dose rate distribution for the area of the array of $3 \times 3$ gas cavities.

IV) Section 7.4: In this section, an attempt was made to investigate the minimum cavity size that can be used with this type of detector by using $2.5 \mathrm{~mm}$ diameter and $1 \mathrm{~mm}$ diameter right cylinder gas cavities. 


\subsection{Single Gaseous Cavity Detector}

The results presented in this section were conducted using the experimental set up explained in section 4.2.1. To investigate the detector operating voltage, the detector was irradiated by a neutron beam resulting from the ${ }^{7} \mathrm{Li}(\mathrm{p}, \mathrm{n})$ reaction. With the beam on, the Cathode and THGEM applied high voltages were increased gradually and the preamplifier and amplifier signals were observed on the oscilloscope. Once the applied voltages were increased enough that the detector reached its proportional region and the signal to noise ratio was noticeably satisfying (sufficiently large for the signal to be clearly distinguishable), raising of the applied voltages was stopped to prevent any possible discharge and breakdown.

Through multiple measurements, it was found out that the first signal of the detector appeared at $700 \mathrm{~V}$ and the maximum safe high voltage was about $1150 \mathrm{~V}$ for the old THGEMs and $1020 \mathrm{~V}$ for the new THGEMs. The reason that the maximum applied voltage across the new THGEMs was lower than the old THGEMs is due to the fact that the old ones had a $0.1 \mathrm{~mm}$ etched rim in the copper surrounding each hole. It was previously shown that THGEMs having a rim could tolerate higher applied voltage and could achieve a higher gain (Breskien et al., 2009; Orchard, 2010). However, it is difficult for the manufacturing companies to fabricate uniformly concentric rims and holes over the entire active area. Therefore rim-hole eccentricity is the major cause of spark-induced damage. This was experimentally observed with one of the THGEMs from the old batch as is shown in Fig. 7.1. Part $f$ clearly shows that the rim-hole eccentricity was the cause of spark-induced damage. Since the high voltage was not reduced immediately after the first indication of a spark, the top (Rexolite) and bottom (readout board) layers were burnt at that spot as well (see Fig. $7.1 b$ and $d$ ). Thereafter, with the first indication of a spark, the applied high voltage was reduced immediately to prevent further damages to the other layers.

After several discussions with PCB manufacturer in Toronto (Milplex Circuit Canada Inc.), it was found out that even with their maximum care and precision in fabricating a clean hole with a concentric rim there were still a few eccentric rim-holes over the active area, which cause operating issues. Hence it was decided to order THGEMs 
without any rim and increase the voltage more cautiously. All THGEMs from the new batch did not have a rim.

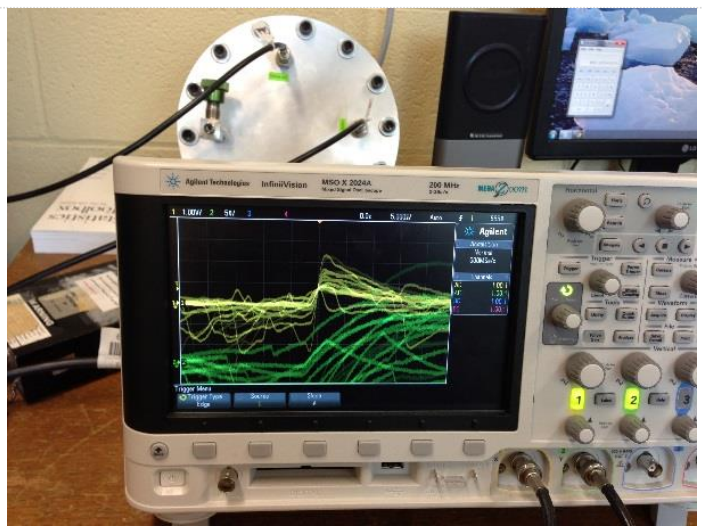

a.

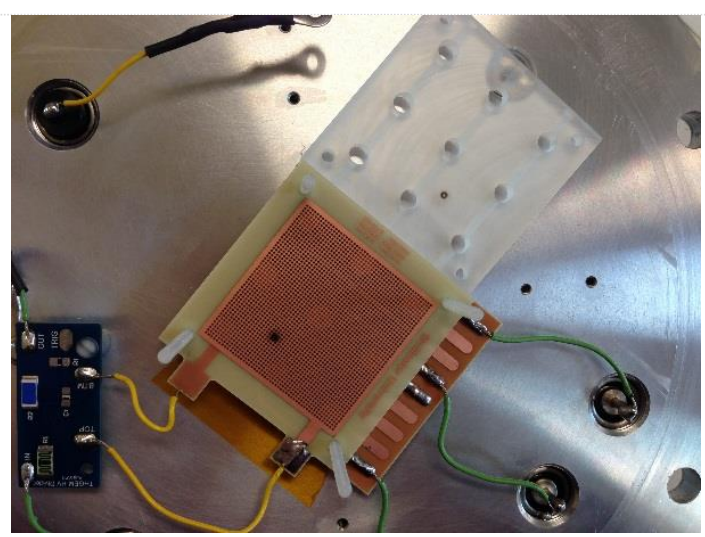

c.

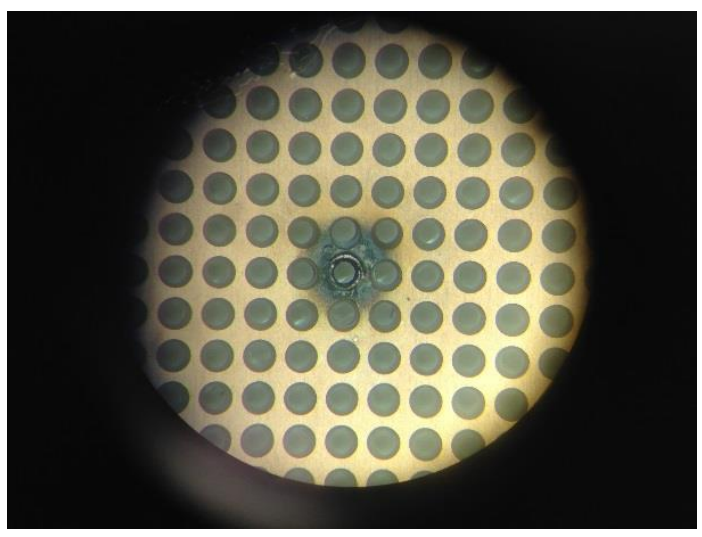

e.

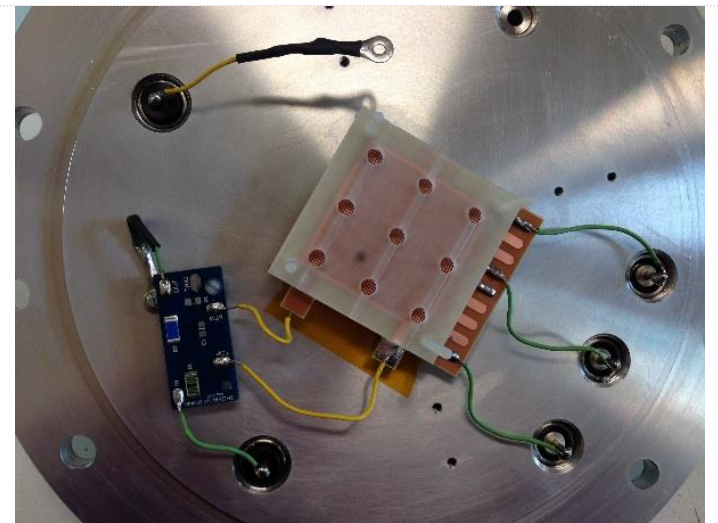

b.

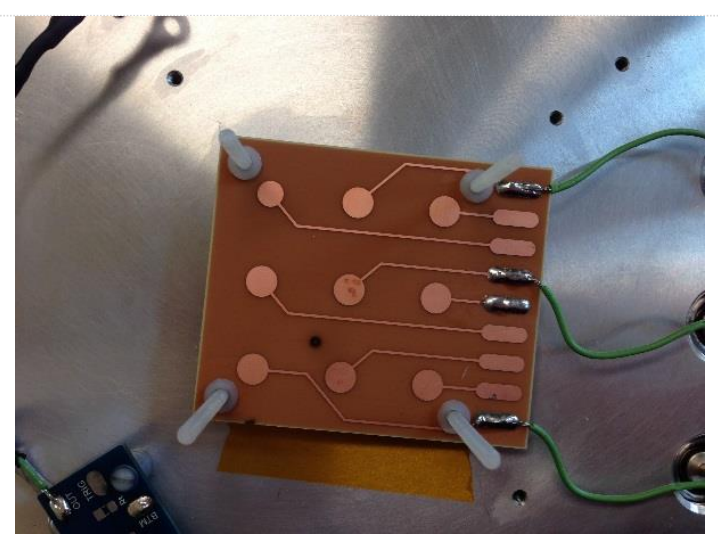

d.

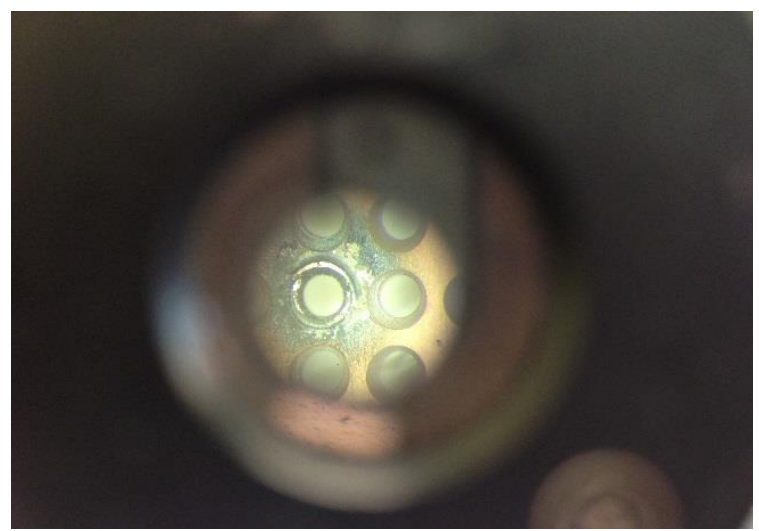

f.

Fig. 7.1 a. An indication of discharge on oscilloscope, b. burnt Rexolit, c. burnt THGEM, d. burnt readout board, e. burnt THGEM hole, f. rim-hole eccentricity 


\subsubsection{Signal Performance}

In order to explore the multiplication gain dependence on the applied voltage across the THGEM, the experimental setup explained in subsection 4.2.1 was employed. The proton energy and current were set to $2.3 \mathrm{MeV}$ and $50 \mu \mathrm{A}$. By increasing the applied voltage to the cathode and THGEM gradually, the growth of the detector signal was observed. Fig. $7.2 b-f$ show the growth of the signal observed on the oscilloscope for 5 different applied high voltages across the THGEM. In any set of measurement, the noise level was initially observed on the oscilloscope and recorded as shown in Fig. 7.2 a. Therefore the scope trigger was set above this level for indication of signal appearance.

For instance, as can be seen in table 7-1 and Fig. 7.3, by applying $760 \mathrm{~V}$ to the THGEM, using eq. 2-1, the voltage difference between the top and bottom of the THGEM would be $633.33 \mathrm{~V}$ and the detector signal is very small $(5 \mathrm{mV})$. By raising the applied THGEM high voltage about $100 \mathrm{~V}$ the detector signal increased by a factor of 4 . By gradually increasing the voltage, it was observed that the detector signal at the $\mathrm{HV}_{\text {THGEM }}=1020 \mathrm{~V}$ was 48 times larger than at $\mathrm{HV}_{\text {THGEM }}=760 \mathrm{~V}$. Fig. 7.3 shows the plot of detector signal amplitude as the high voltage applied across the THGEM was increased.

Table 7-1. Applied high voltages and corresponding detector signal amplitude data

\begin{tabular}{cccccc}
\hline HV & \\
CVathode $_{\text {[V] }}$ & 860 & 960 & 1020 & 1080 & 1120 \\
\hline$\Delta \mathbf{V}_{\text {THGEM }}[\mathrm{V}]$ & 760 & 860 & 920 & 980 & 1020 \\
Signal $_{\text {detector }}[\mathrm{mV}]$ & 633 & 717 & 767 & 817 & 850 \\
\hline
\end{tabular}




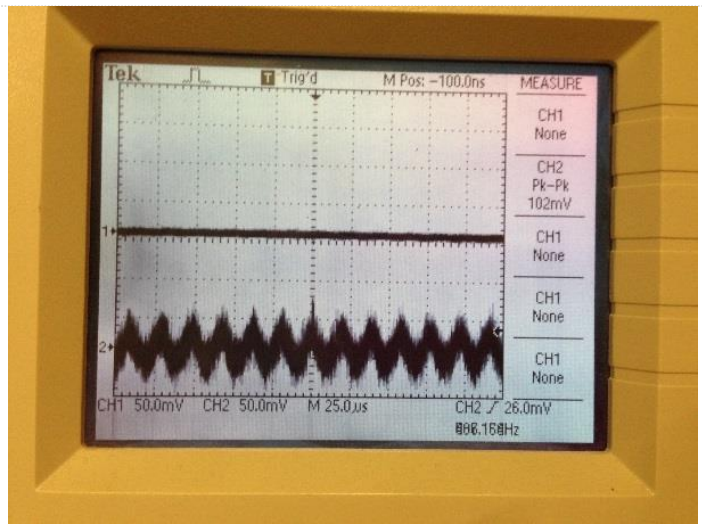

a.

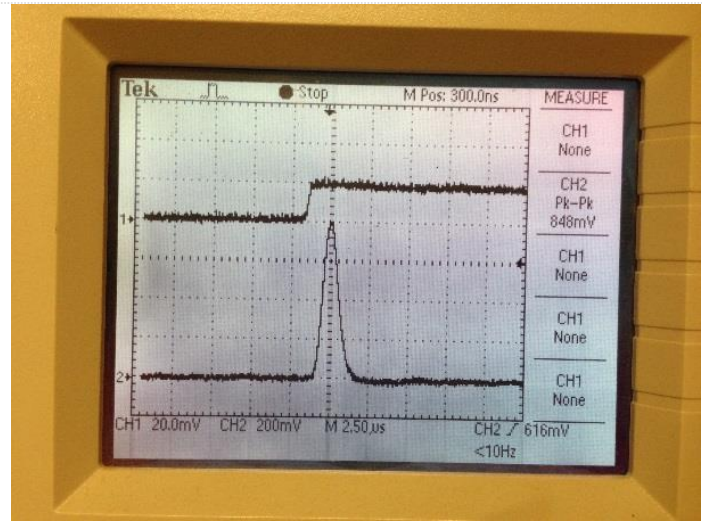

c.

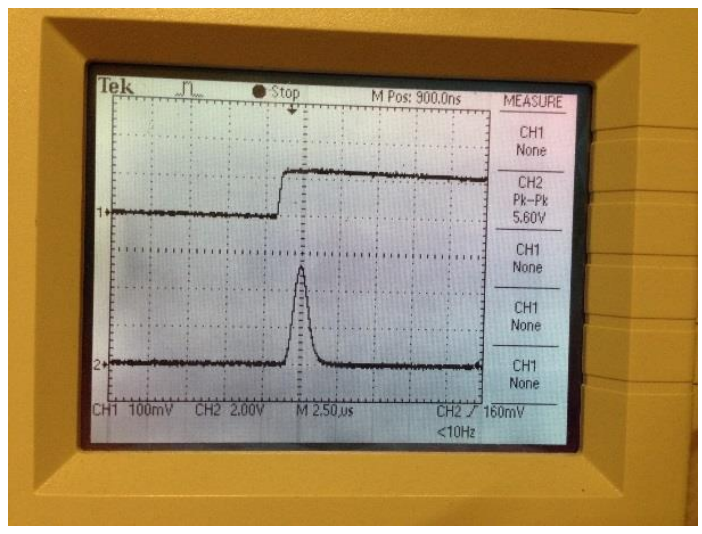

e.

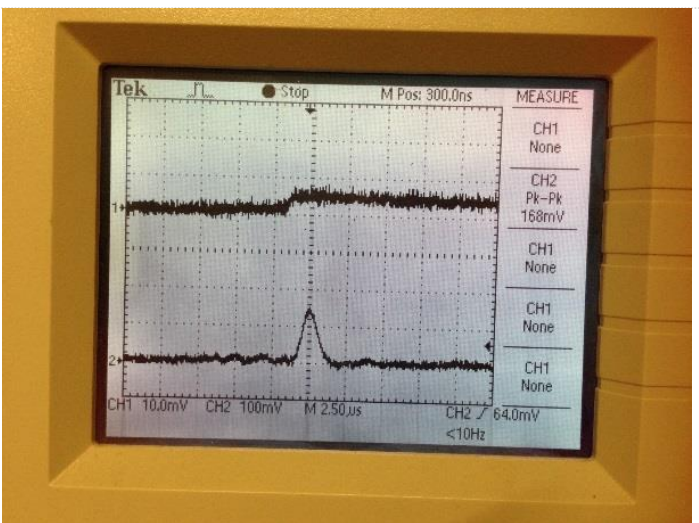

b.

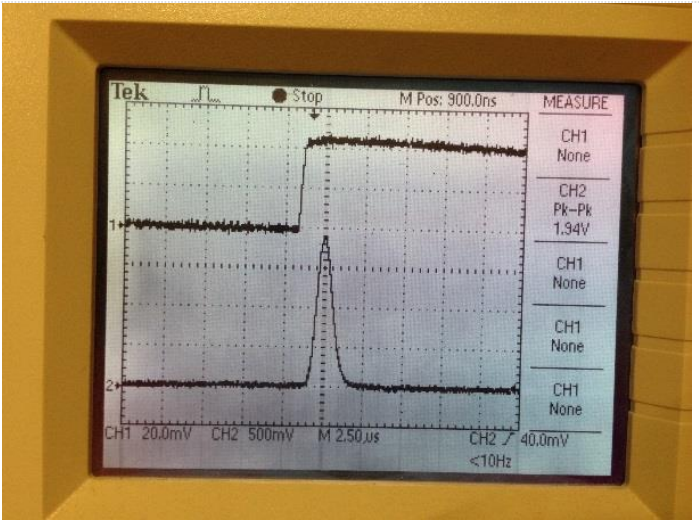

d.

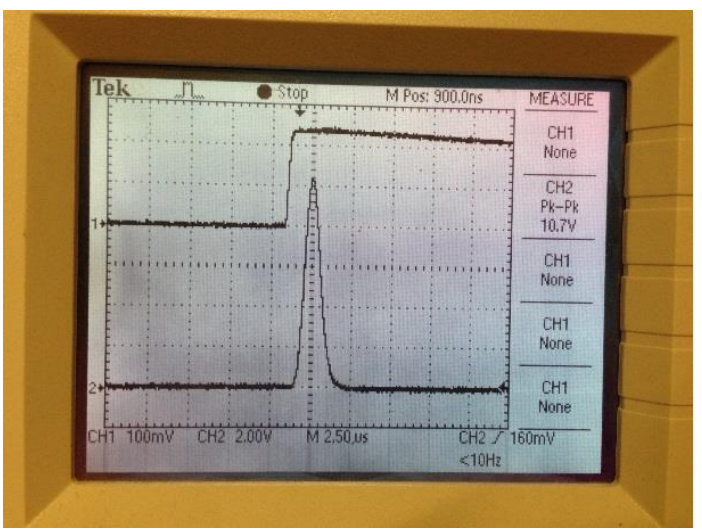

f.

Fig. 7.2 Growth of the preamplifier (CAEN) (channel 1) and amplifier (gain of 50) (channel 2) signal on the oscilloscope with increasing the THGEM applied voltage a. Noise level without beam, $\mathbf{b}$. $H V_{\text {THGEM }}=$ $760 \mathrm{~V}$, c. $\mathrm{HV}_{\mathrm{THGEM}}=860 \mathrm{~V}$, d. $\mathrm{HV}_{\mathrm{THGEM}}=920 \mathrm{~V}$, e. $\mathrm{HV}_{\mathrm{THGEM}}=980 \mathrm{~V}$, f. $\mathrm{HV}_{\mathrm{THGEM}}=1020 \mathrm{~V}$ 


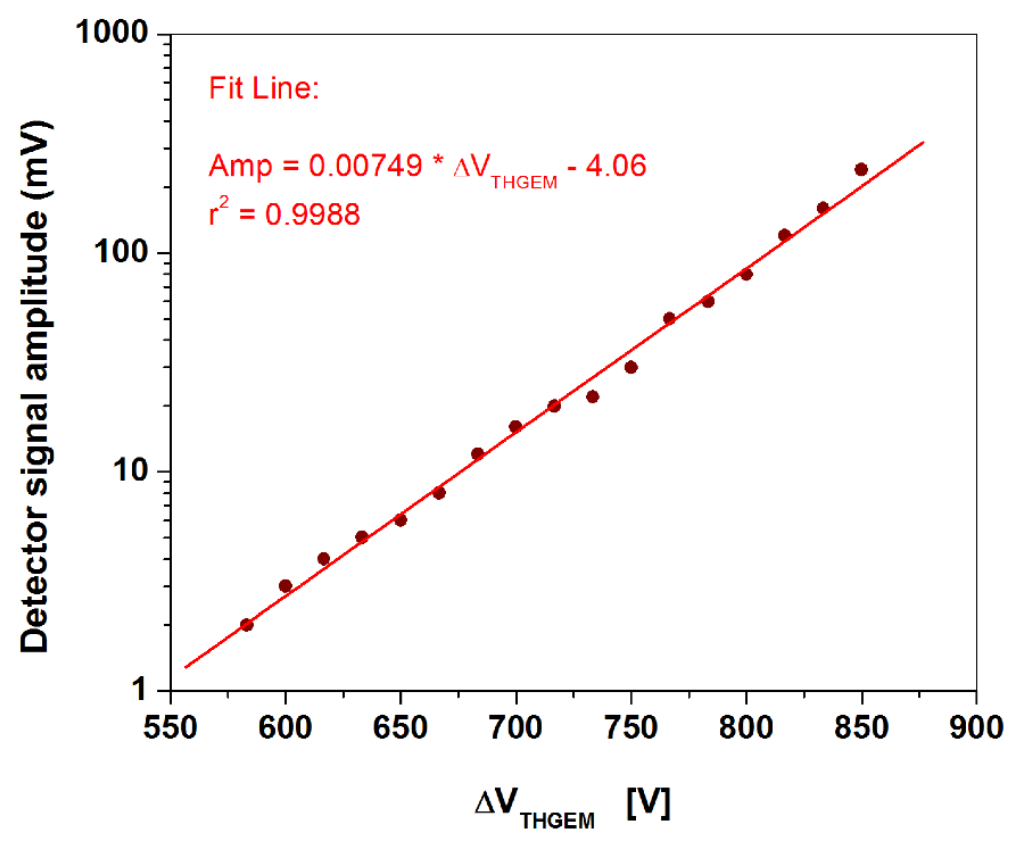

Fig. 7.3 Detector signal amplitude vs. applied high voltage across the THGEM

\subsubsection{Stability and Reproducibility}

\section{Old THGEMs:}

The detector stability was investigated by collecting data every $300 \mathrm{sec}$ for 6 cycles. Fig. 7.4 shows the raw spectra collected within a day of measurement using one of the old THGEMs batches. As can be seen, there is not a significant gain shift within half an hour measurement time. After 2 hours the detector stability was tested one more time to explore any gain shift. As shown in Fig. 7.5, the detector gain is stable within half an hour period after 2 hours measurement. 


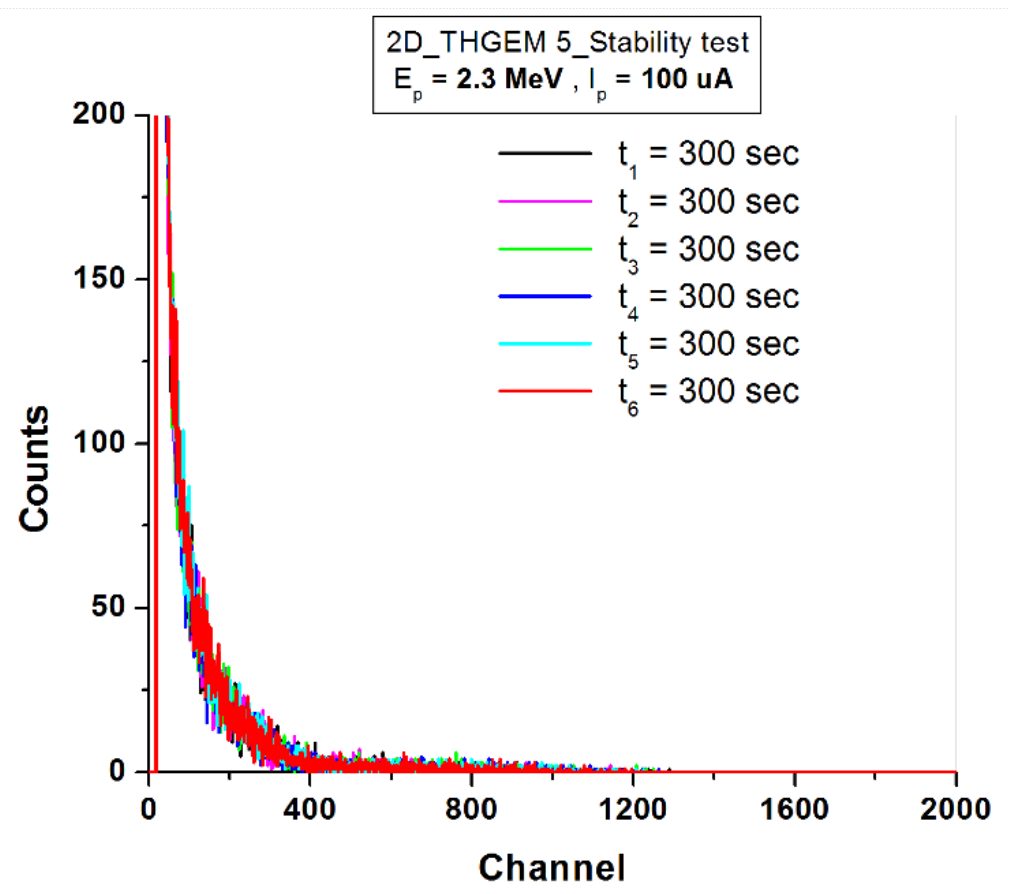

a.

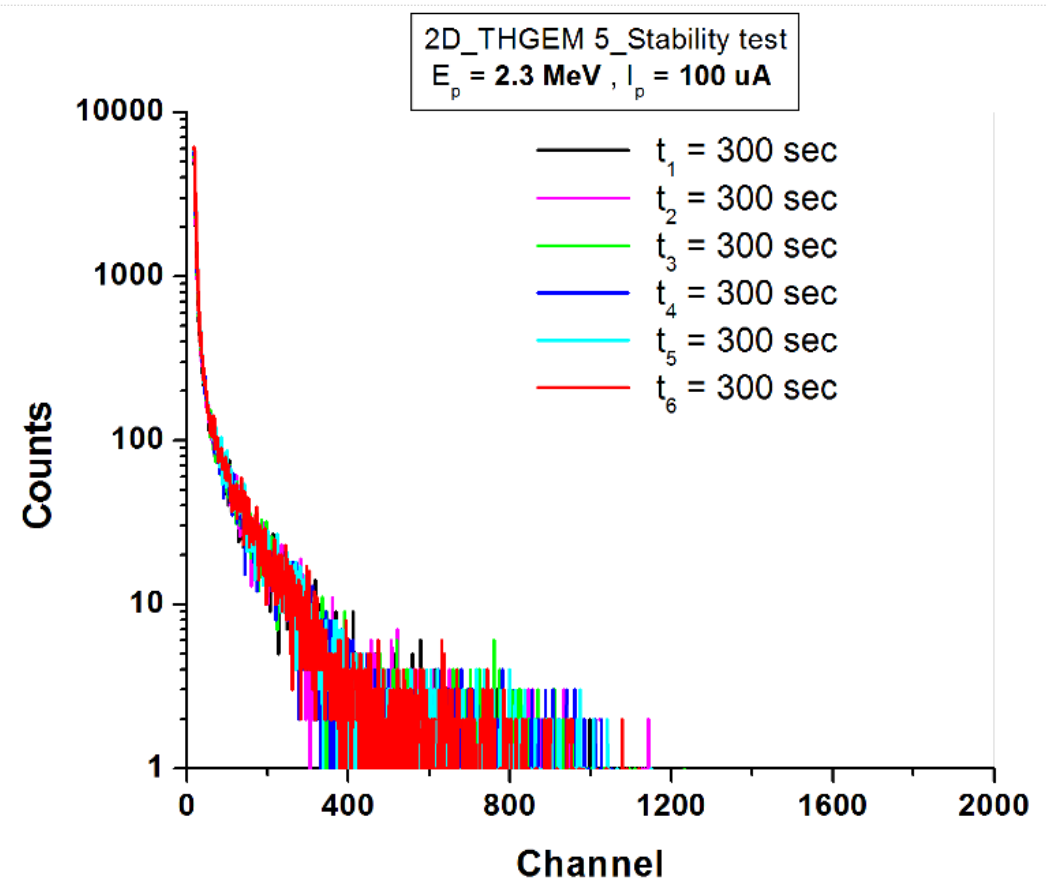

b.

Fig. 7.4 Detector gain stability over half an hour time period (27 Nov. 2014 data) a. linear scale, b.log scale 


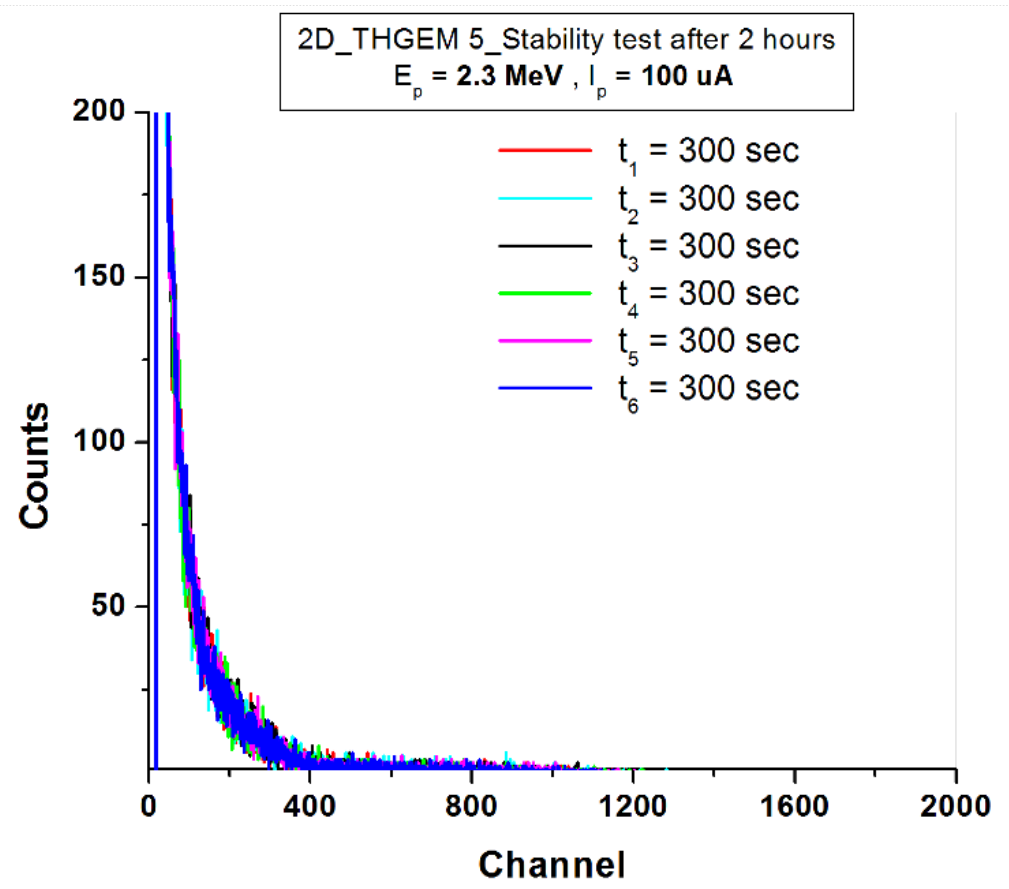

a.

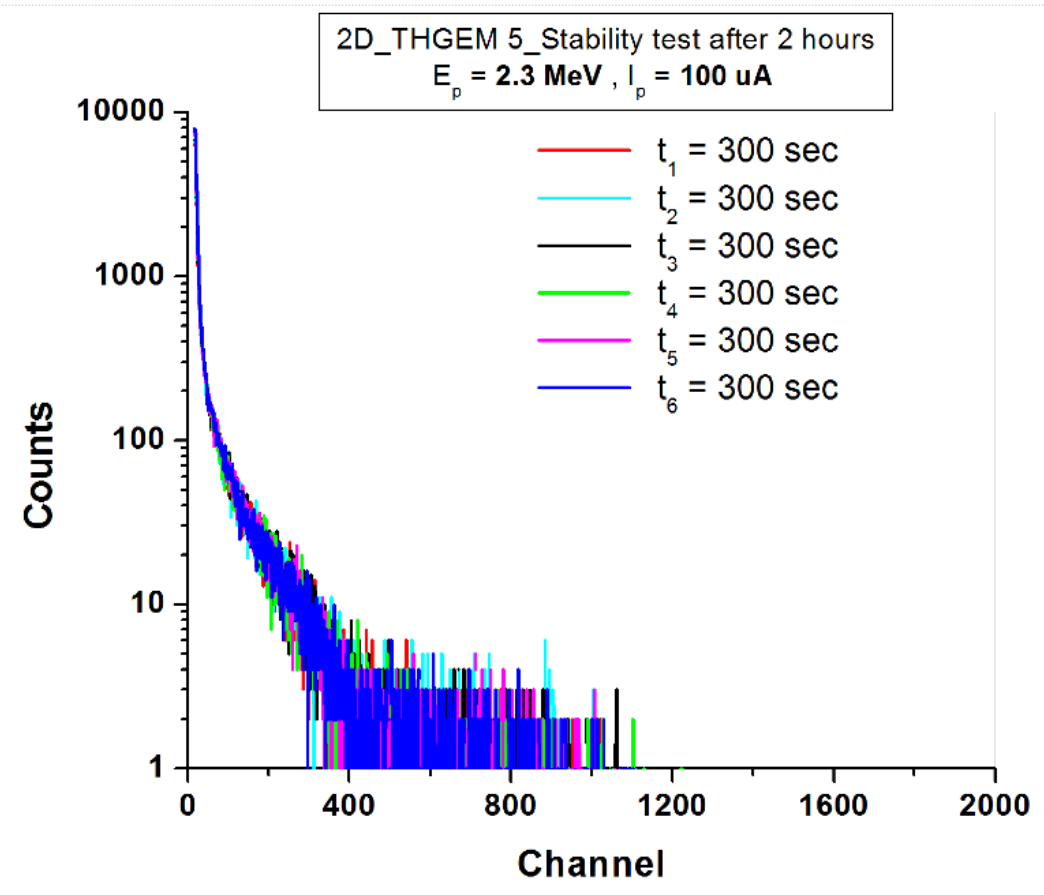

b.

Fig. 7.5 Detector gain stability over half an hour time period after 2 hours from the beginning of measurement (27 Nov. 2014 data) a. linear scale, b. log scale 
For a better conclusion, the spectra at the beginning of measurement and $2 \mathrm{hrs}$ later were plotted together in Fig. 7.6. Clearly, there is no significant gain shift over $2 \mathrm{hrs}$ measurement as well.

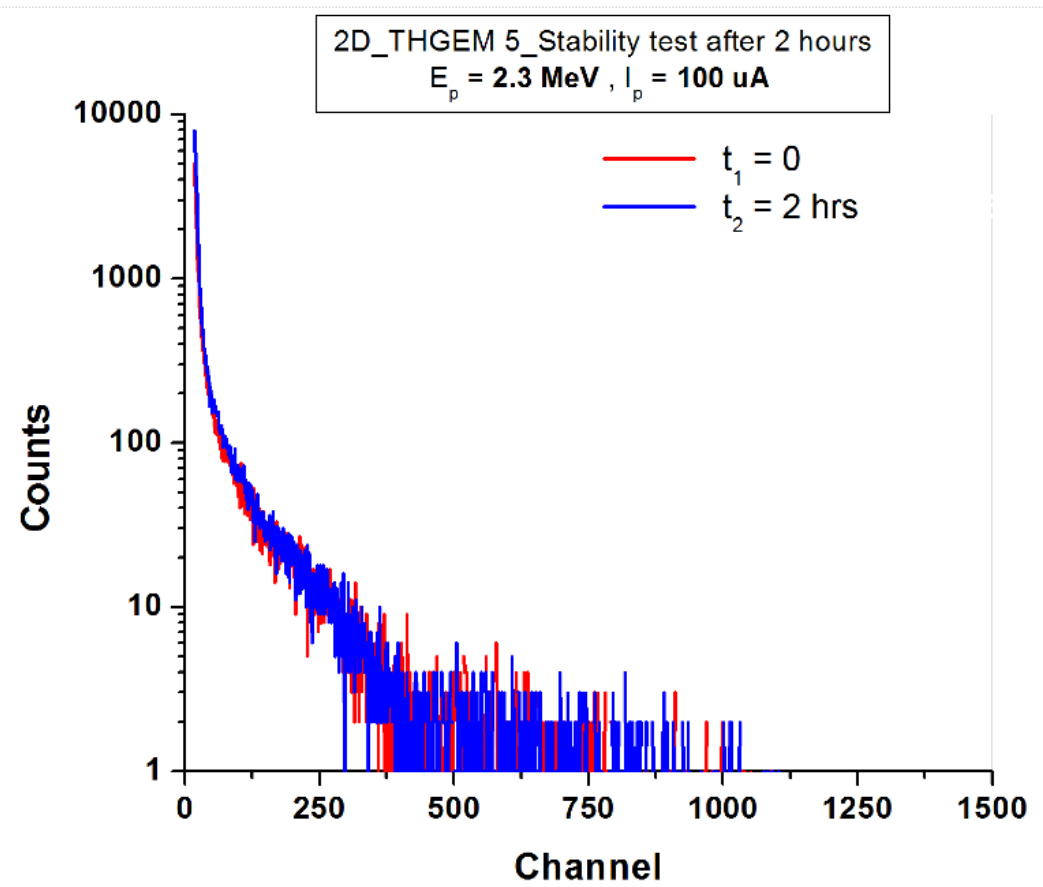

Fig. 7.6 Detector gain stability over 2 hours from the beginning of measurement (27 Nov. 2014 data)

To demonstrate the reproducibility of data, a set of measurements was performed on another day about 3 weeks later. All detector features and measurement settings were kept the same. Fig. 7.7 illustrates the spectra collected on two different days: 27 Nov. 2014 and 16 Dec. 2014. Quantitatively comparing the two sets of data it was found out that the area under the 27 Nov. data was $20 \%$ higher than that of 16 Dec. It was found that this was due to the higher noise level on that particular day (27 Nov.). In general, the areas must be determined above the noise level so this is not a significant issue.

The stability results were reproduced over repeated measurements with different good working THGEMs. 


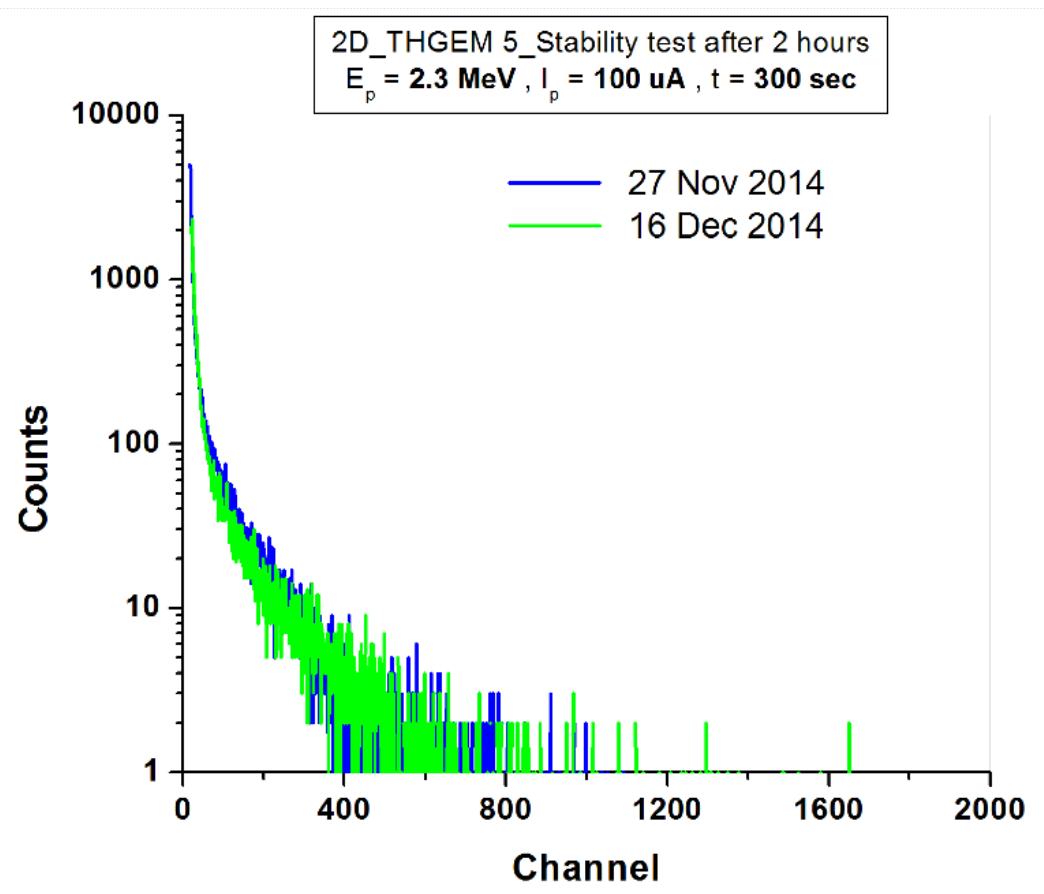

Fig. 7.7 Detector gain stability over 3 weeks

\section{New THGEMs:}

The stability test was carried out for the new batch of THGEMs from MILPLEX Company in Toronto as well. For the first THGEM that was installed from the new batch the first indication of signal happened at around $\mathrm{HV}_{\text {THGEM }}=500 \mathrm{~V}$ which was about 200 $\mathrm{V}$ lower than that for old THGEMs, meaning that the amendment that had been made in the fabrication process of the new batch had made a significant improvement on the THGEM gain. However, the detector had a spark when the THGEM applied high voltage was raised to $750 \mathrm{~V}$. The raw data collected for $277 \mathrm{sec}$, at proton energy and current of $2.3 \mathrm{MeV}$ and $50 \mu \mathrm{A}$ are shown in Fig. 7.8. When the detector was opened, it was observed that there were spots of liquid or oil on the THGEM layer as shown in Fig. 7.9 which were not there at the time of installation. The only reason that this could be explained is that during the fabrication process the cleaning was not done thoroughly. Therefore the THGEM was replaced with another good looking one for the next measurement. This issue did not happen anymore with any other THGEM from the new batch. 


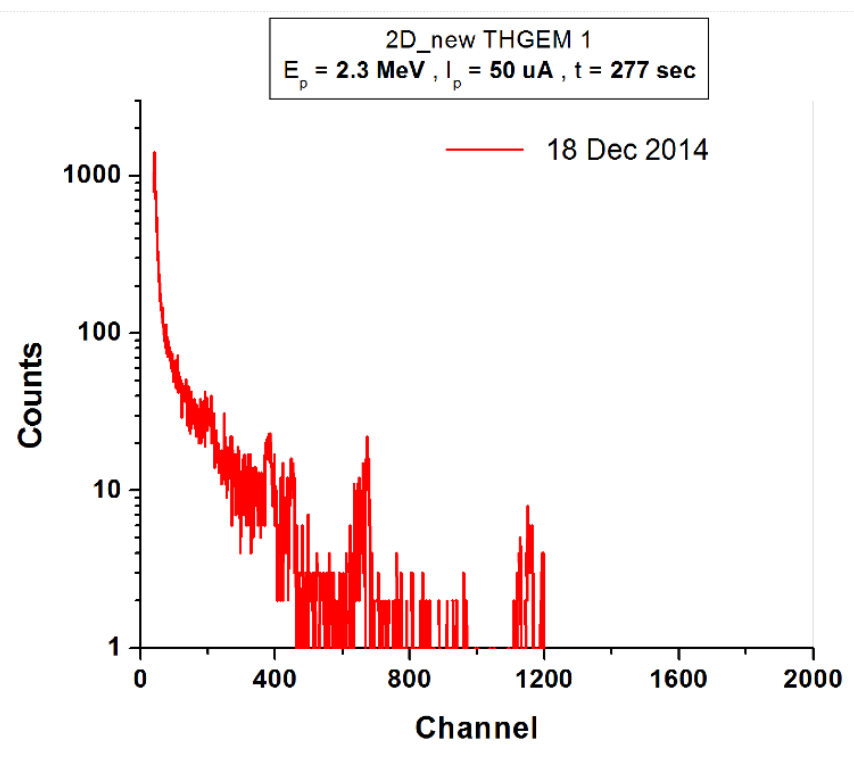

Fig. 7.8 Raw spectrum of new THGEM \#1 which was sparked

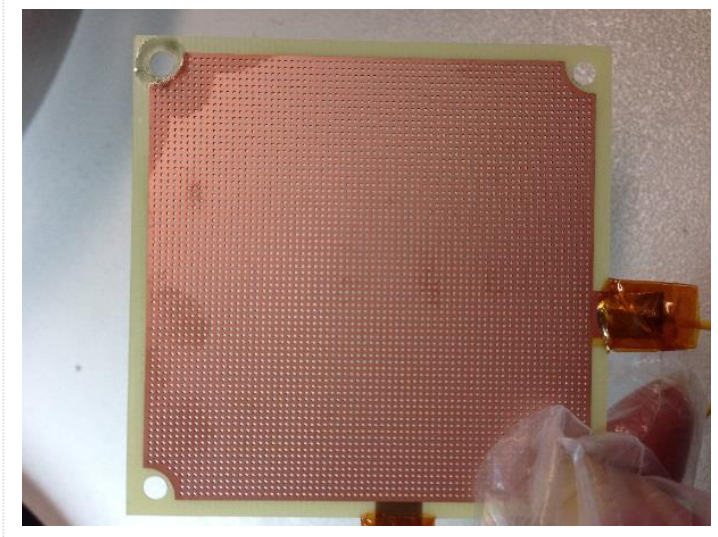

Fig. 7.9 Liquid/oil spots on THGEM \#1 from a new batch caused spark during measurement

Fig. 7.10 shows the stability test of the detector with new THGEM \#5 which was one of the good looking THGEMs. As can be seen, all four spectra collected for $300 \mathrm{sec}$ were consistent and there is no significant gain shift over a 20 min time period.

The stability of the detector was tested over a two-week time period for the same condition. The stability result is shown in Fig. 7.11. 


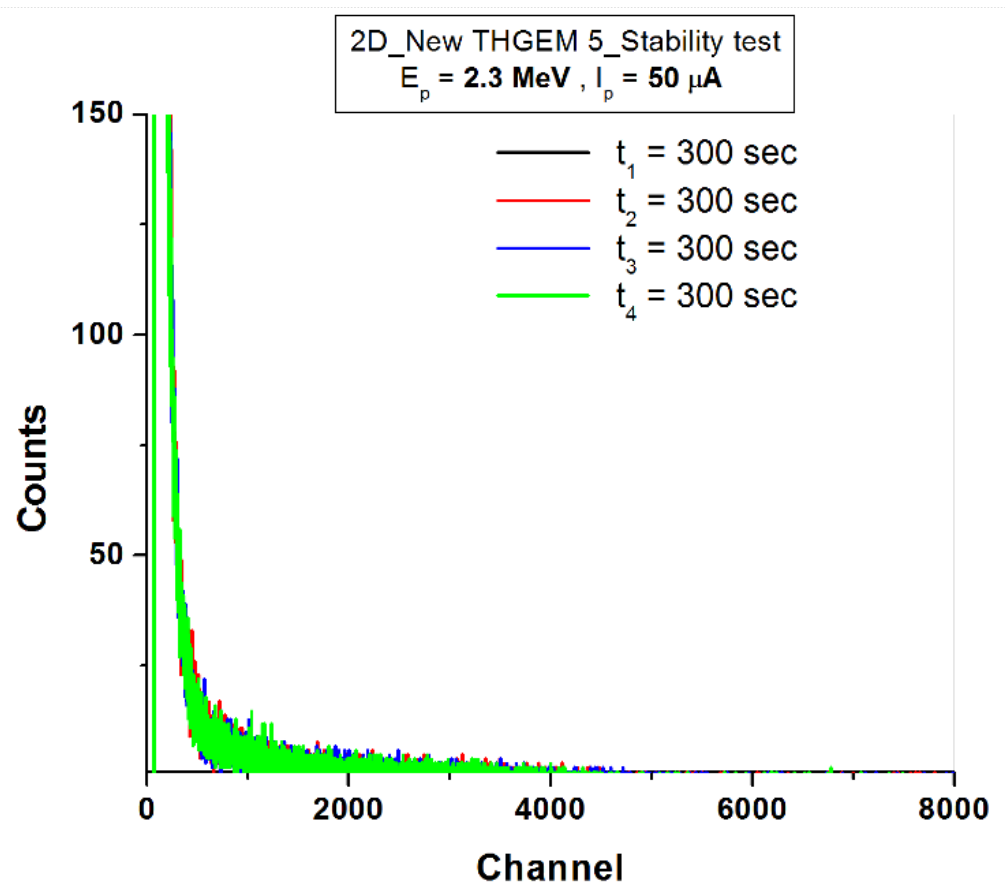

a.

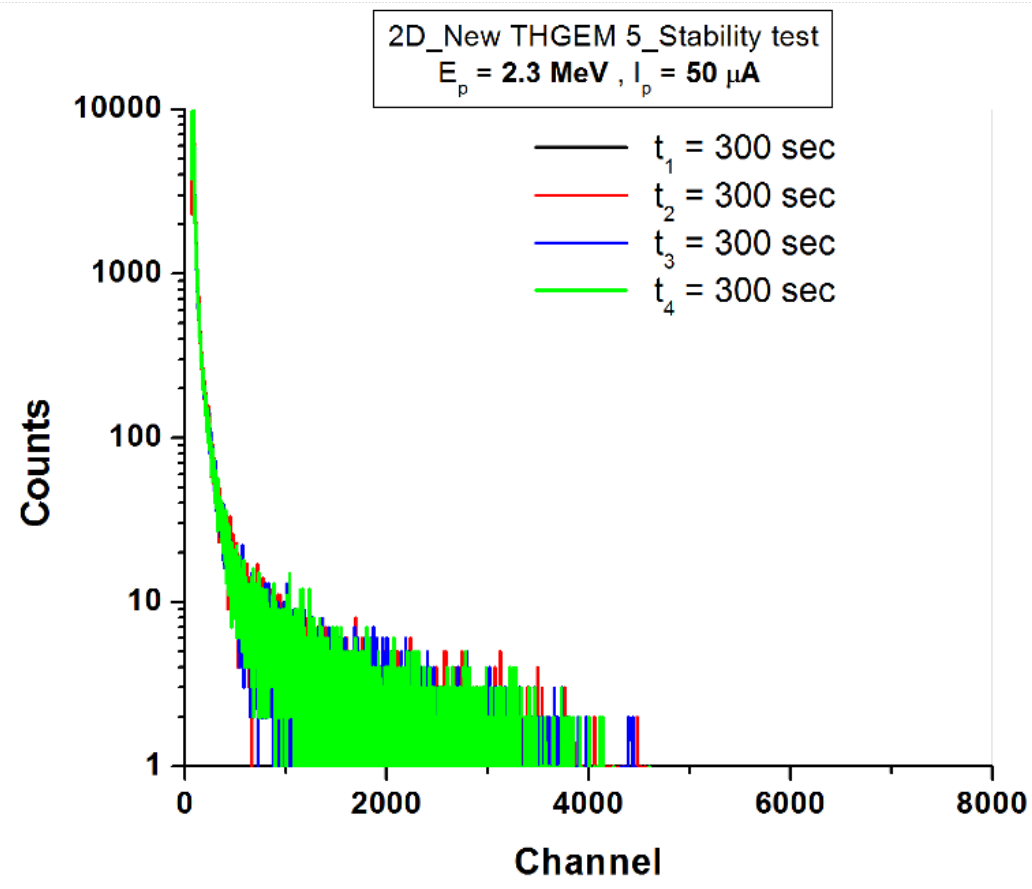

b.

Fig. 7.10 Detector gain stability over 20 minutes time period a. linear scale, b.log scale 


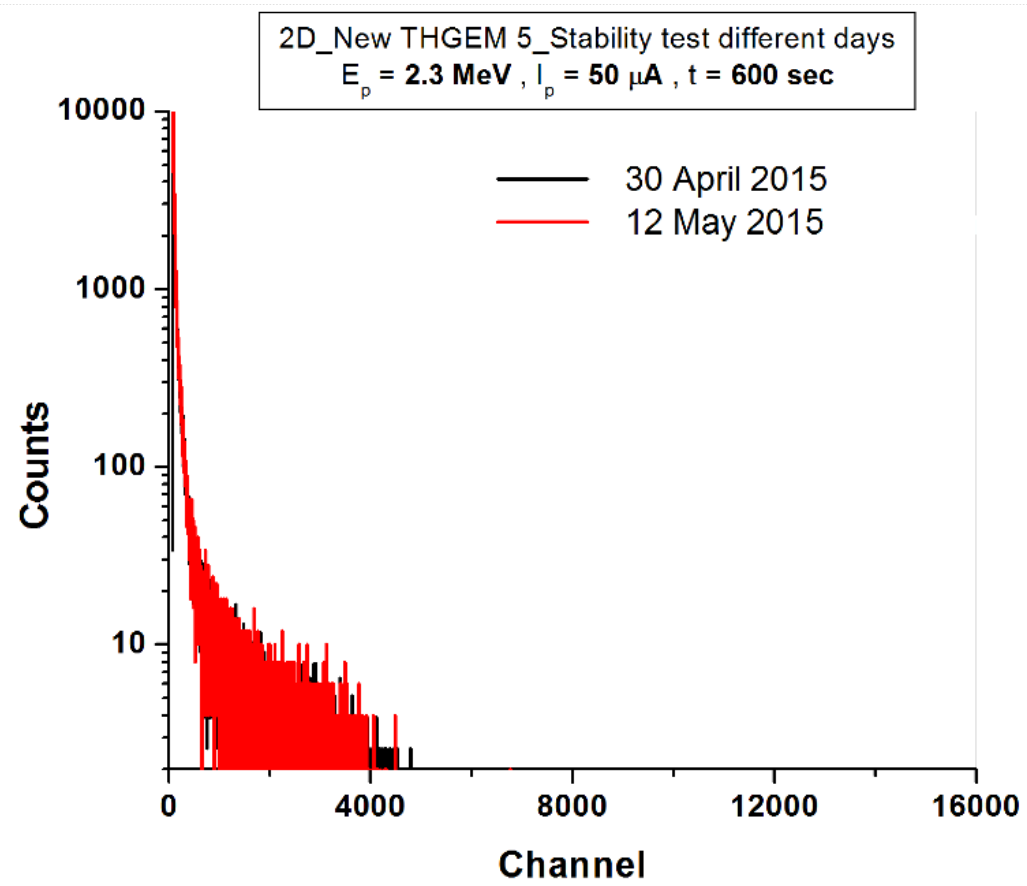

a.

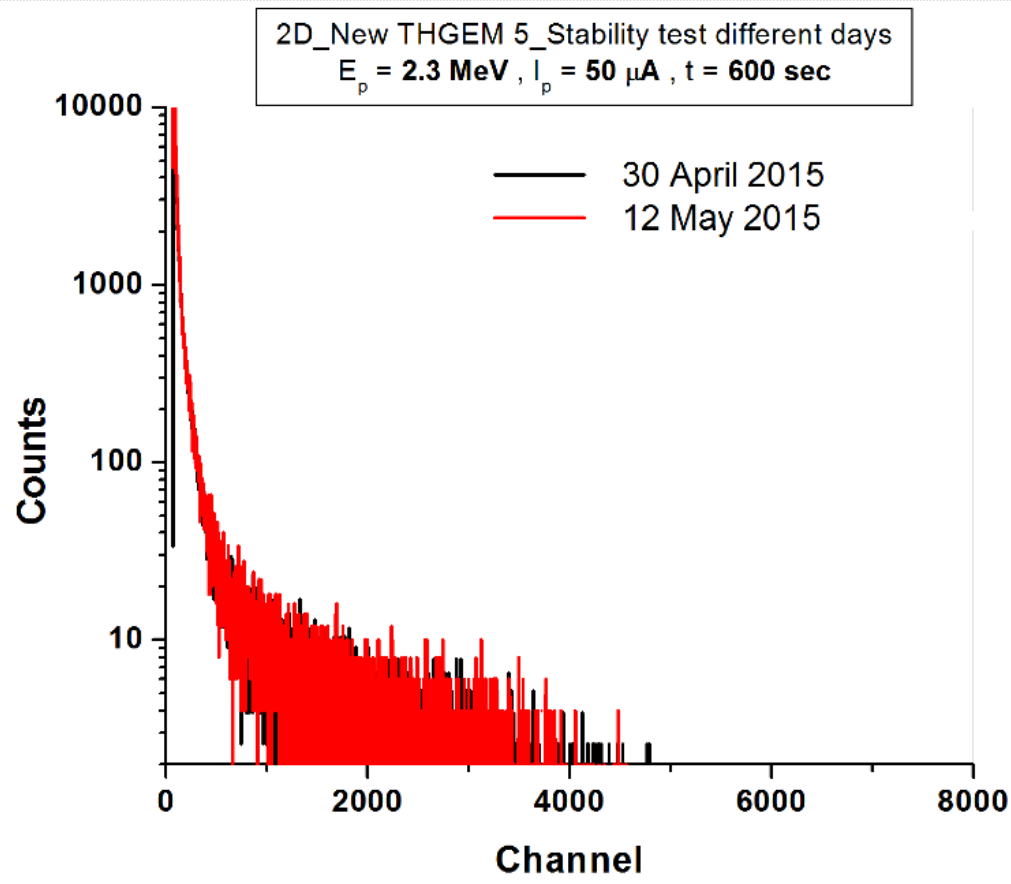

b.

Fig. 7.11 Detector gain stability over 2 weeks a. Full scale, b. Zoom in 


\subsubsection{Microdosimetric Spectrum}

The microdosimetric spectrum of single gaseous cavity THGEM detector was investigated using THGEMs from old and new batches. Data analysis methodology and how to calibrate the lineal energy to obtain a microdosimetric spectrum was fully explained in chapter 5 .

\section{Old THGEMs:}

Fig. 7.12 presents the microdosimetric spectra obtained with various old THGEMs and compared to the microdosimetric spectra obtained with a commercial 1/2" TEPC and a 2" TEPC at the THGEM detector position inside the irradiation cavity (see Fig. 4.3). For a better visual comparison and to eliminate the effect of different experimental conditions the vertical scale is quoted in terms of $\mathrm{yN}(\mathrm{y})$ normalised by the total proton charge incident on the lithium target and the mass of the detector as explained in chapter 5.

In this Fig. the microdosimetric spectra for old THGEM \# 1, obtained on two different days (28 May 2014 and 8 Oct 2014) but under the same experimental conditions were compared. As can be seen, the two spectra are consistent. Compared to the microdosimetric spectrum of the 1/2" TEPC, clearly, they are in agreement for the gamma and neutron parts. In both cases, proton peaks were matched. However, there is a discrepancy in the region of the spectra at lineal energies greater than $150 \mathrm{keV} / \mu \mathrm{m}$. There appears to be another peak generated after the neutron peak, the amplitude of which usually varied for different THGEMs. This was observed by Orchard (2010) as well. THGEM \#2, \#3, \#4, \#6, \#7 and \#8 were excluded from the results as they were not working properly and a reasonable signal could not be achieved. 


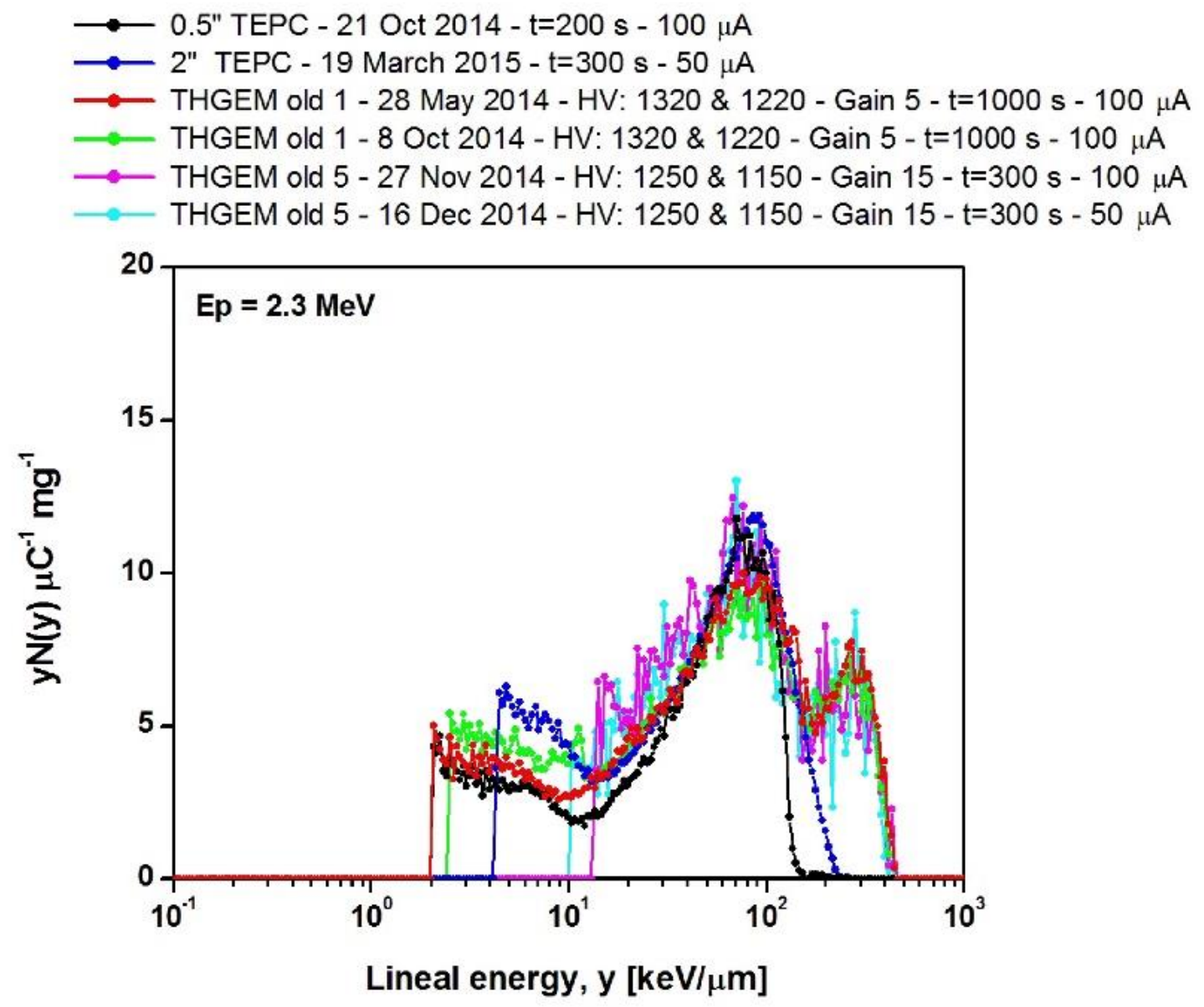

Fig. 7.12 THGEM detector microdosimetric spectrum using different THGEMs from old batch compared to commercial 1/2" TEPC and 2" TEPC

The spectra obtained on 27 Nov 2014 and 16 Dec 2014 with THGEM \#5 are shown in Fig. 7.12 as well. The experimental conditions were slightly different from the other two measurements carried out on 28 May 2014 and 8 Oct 2014. The applied high voltage was $70 \mathrm{~V}$ less, as this THGEM could not bear the same high voltage as the previous ones. Therefore the detector was not able to detect lower energy events as expected. The acquisition time was $300 \mathrm{sec}$ compared to $1000 \mathrm{sec}$ for the previous measurements. This could explain the poor statistics of the spectra with THGEM \#5 when compared with the 
THGEM \#1. The ADC was set to 2k channels for the data acquired on 27 Nov 2014 and 16 Dec 2014 compared to 16k channels for the data acquired on 28 May 2014 and 8 Oct 2014. This is another factor that causes the statistical fluctuations in spectra, as the number of bins per decade becomes much smaller as the lineal energy decreases. In other words, not enough data points fall into the specific bin for $2 \mathrm{k}$ channels compared to $16 \mathrm{k}$. This fact was investigated in detail and will be presented later on, in this section.

Evidently, the higher events that appeared after the proton edge were generated with THGEM \#5 as well. This could be justified as the non-uniform gas multiplication throughout the THGEM holes. As the THGEMs fabricated for this research had about 150 times more holes than the previous study (Orchard, 2010) there could be much more holes which were not drilled uniformly. Besides the quality control of 5000 holes is much more difficult. All these factors could end up with a non-uniform gas multiplication which causes a broader neutron peak and possibly the second peak.

\section{New THGEMS:}

To investigate the causes of generation of events after the proton edge, the detector was tested using THGEMs from the new batch. As was previously explained in section 2.4, the new THGEMs were fabricated more precisely and more polishing and deburring processes were performed by the manufacturer. As was mentioned in the previous section, the first THGEM tested from the new batch did not work properly and sparked with increasing the high voltage. Fig. 7.13 shows the microdosimetric spectra obtained with various new THGEMs and compared to the microdosimetric spectra obtained with a commercial 1/2" TEPC and 2" TEPC at the THGEM detector position inside the irradiation cavity (see Fig. 4.3). Experimental settings are pointed out in the legend of the plot. As was discussed previously, the new THGEMs did not have a rim around each hole, thus the maximum applied high voltage was lower than that of old THGEMs. The maximum high voltage that could be applied was $1120 \mathrm{~V}$ to the cathode and $1020 \mathrm{~V}$ to the THGEM. 


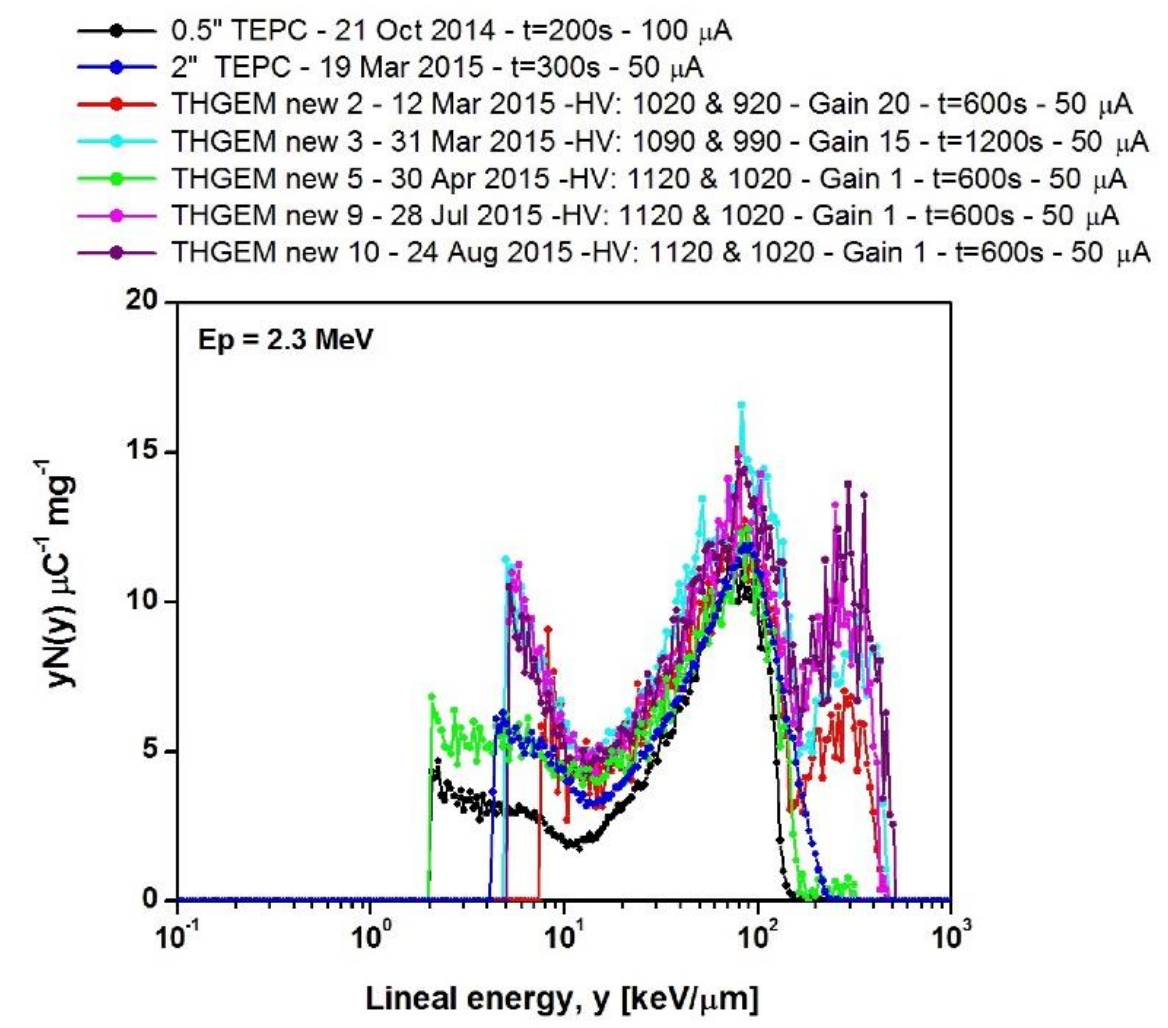

Fig. 7.13 THGEM detector microdosimetric spectrum using different THGEMs from new batch compared to commercial 1/2" TEPC and 2" TEPC

To investigate the effect of applied high voltage across the THGEM on the presence of the second peak, it was attempted to reduce the voltage across the THGEM for THGEMs \#2 and \#3. Reducing the voltage might help if high amplitude pulses appear due to some sort of sparks across the holes of the THGEM. Comparing spectra obtained with THGEM \#2 and \#3 on 12 March 2015 and 31 March 2015, respectively, clearly the second peak is smaller for THGEM \#2 whose applied high voltage is $70 \mathrm{~V}$ lower. However, the lower lineal energy events were not detectable. From this Fig. it is evident that as the applied high voltage to the THGEM increases, the gas multiplication of lower energy events became above the electrical noise level and thus made these events detectable.

Detector response was also tested using THGEM \#4 but obtaining a reasonable signal with a great signal to noise ratio was impossible. The detector signal was not increased 
accordingly by raising the voltage. Therefore those data were excluded. An interesting result was obtained with THGEM \#5. The response of the detector was fully matched to that of commercial 1/2" TEPC. Evidently, there was no second peak in the microdosimetric spectrum of this THGEM and the neutron peak perfectly matched. The lower lineal energy part of the spectrum is slightly higher than the standard 1/2" TEPC but perfectly matched with the commercial 2" TEPC. This could be due to the fact that the 1/2" TEPC data were acquired in Oct 2014, however, both THGEM \#5 detector and commercial 2" TEPC were acquired within a month in 2015 .

As a summary Fig. 7.14 presents the microdosimetric spectra obtained with the good working THGEMs from old (\#1) and new (\#5) batches. These spectra were plotted versus standard 1/2" TEPC and 2" TEPC.

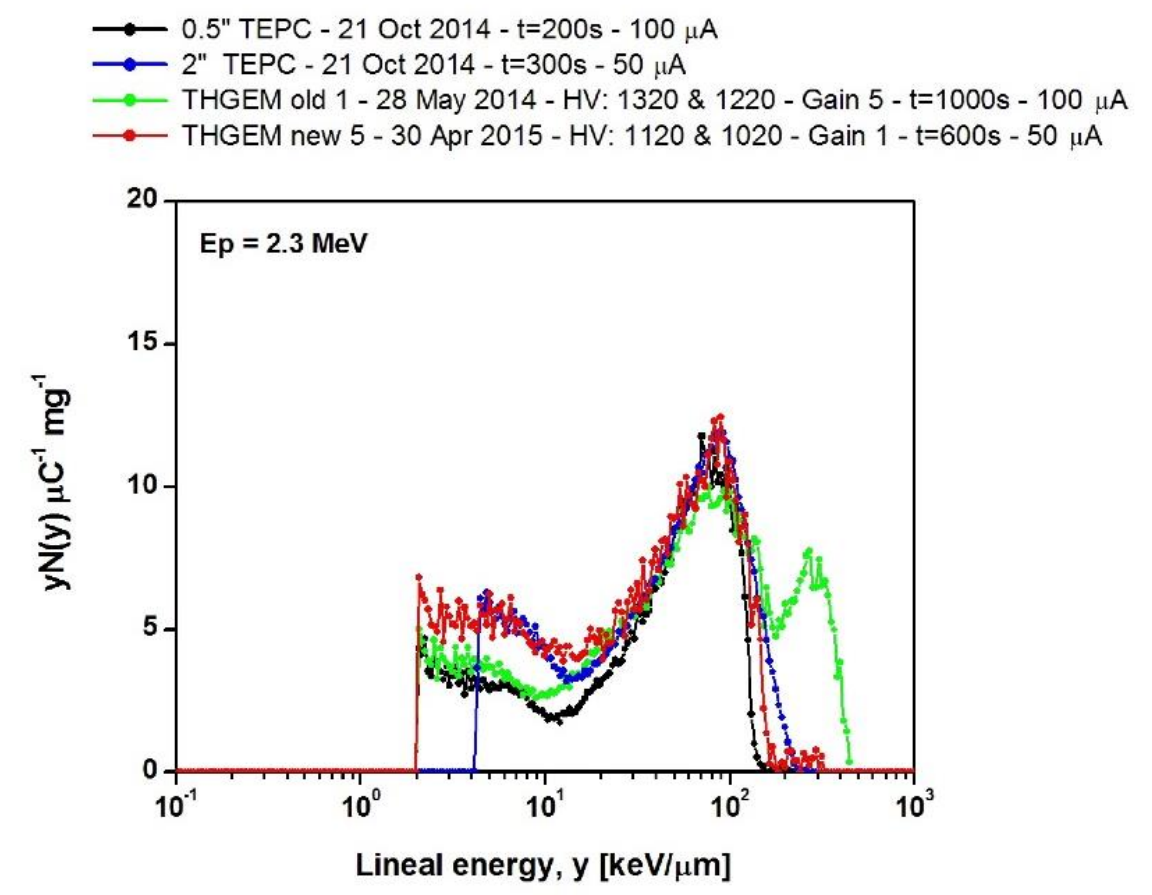

Fig. 7.14 THGEM detector microdosimetric spectrum using THGEMs from old batch vs. THGEM from new batch compared to commercial 1/2" TEPC and 2" TEPC 


\subsubsection{Chemically Cleaned THGEM Data}

In order to have clean and uniform THGEM holes, an attempt was made to clean one of the THGEMs from the new batch chemically using Nitric Acid as shown in Fig. $7.15 \mathrm{a}$. After several trials (different Nitric Acid concentrations over different times), the best result (in terms of visual appearance) was achieved for a 20\% Nitric Acid over 20 min. Then the THGEM was rinsed with distilled water and baked for about a day at $85{ }^{\circ} \mathrm{C}$. The THGEM surface and holes after cleaning are shown in Fig. 7.15 d. Visually, the holes under the microscope looked very clean and uniform without any sharp edges or copper debris.

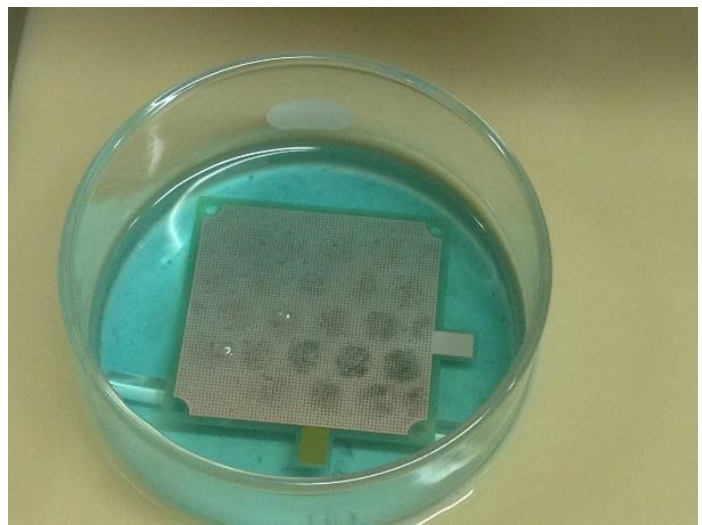

a.

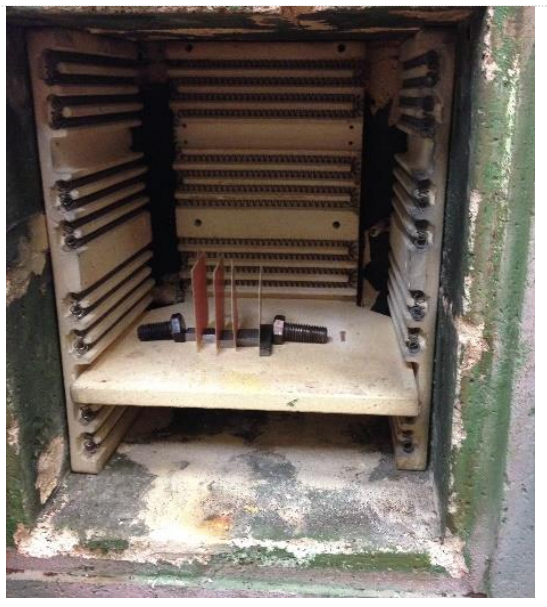

c.

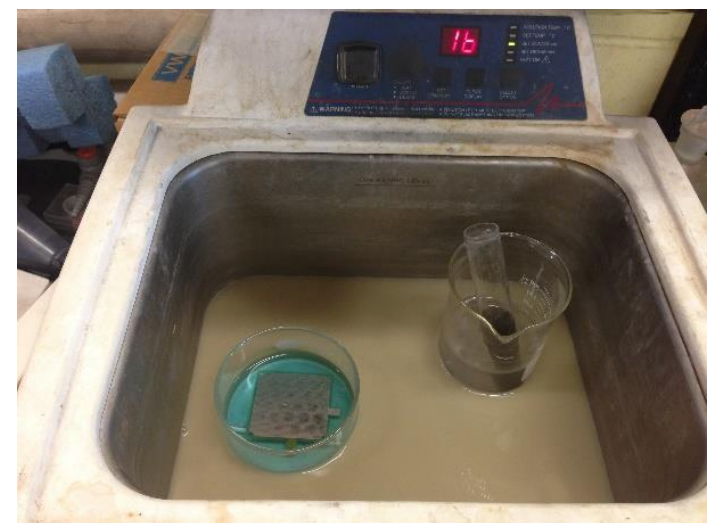

b.

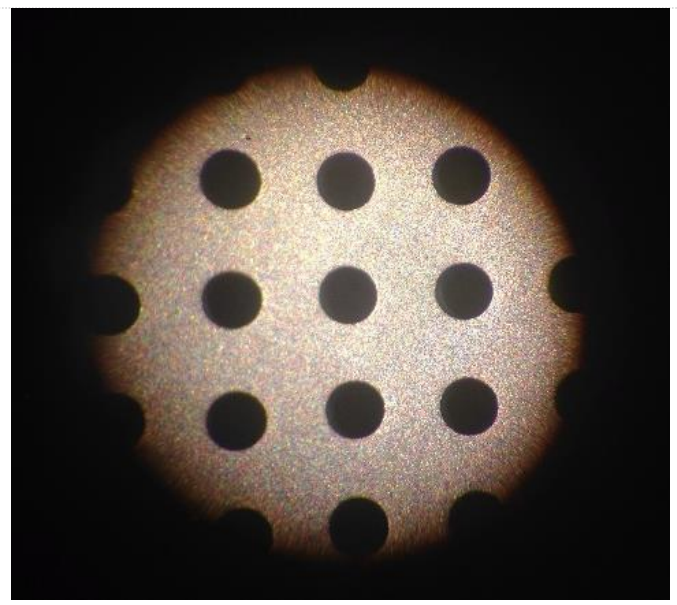

d.

Fig. 7.15 a. A THGEM in 20\% Nitric Acid, b. the THGEM in the Nitric Acid in the ultrasonic bath for 20 min, c. cleaned THGEMs in the oven d. THGEM surface and holes under microscope 
The cleaned THGEM was then installed in the detector and using the same experimental setup as before, the detector response was examined. The applied high voltage could be increased to $1160 \mathrm{~V}$ for the cathode and $1060 \mathrm{~V}$ for the THGEM, which was $40 \mathrm{~V}$ higher than for the previous THGEMs that were not chemically cleaned.

The microdosimetric spectrum for $\mathrm{E}_{\mathrm{p}}=2.3 \mathrm{MeV}$ and $\mathrm{I}_{\mathrm{p}}=50 \mu \mathrm{A}$ is shown in Fig. 7.16. Clearly, no significant improvement was observed for the detector performance and microdosimetric spectrum, except that the THGEM bearable high voltage was increased. This might be favourable when the detector optimization is carried out in order to see more of the lower lineal energy part of the spectrum. However, as there was no indication of a significant difference between the result of the chemically cleaned THGEM and the one that had not been cleaned, the chemical cleaning process was not performed for any other THGEM afterward.

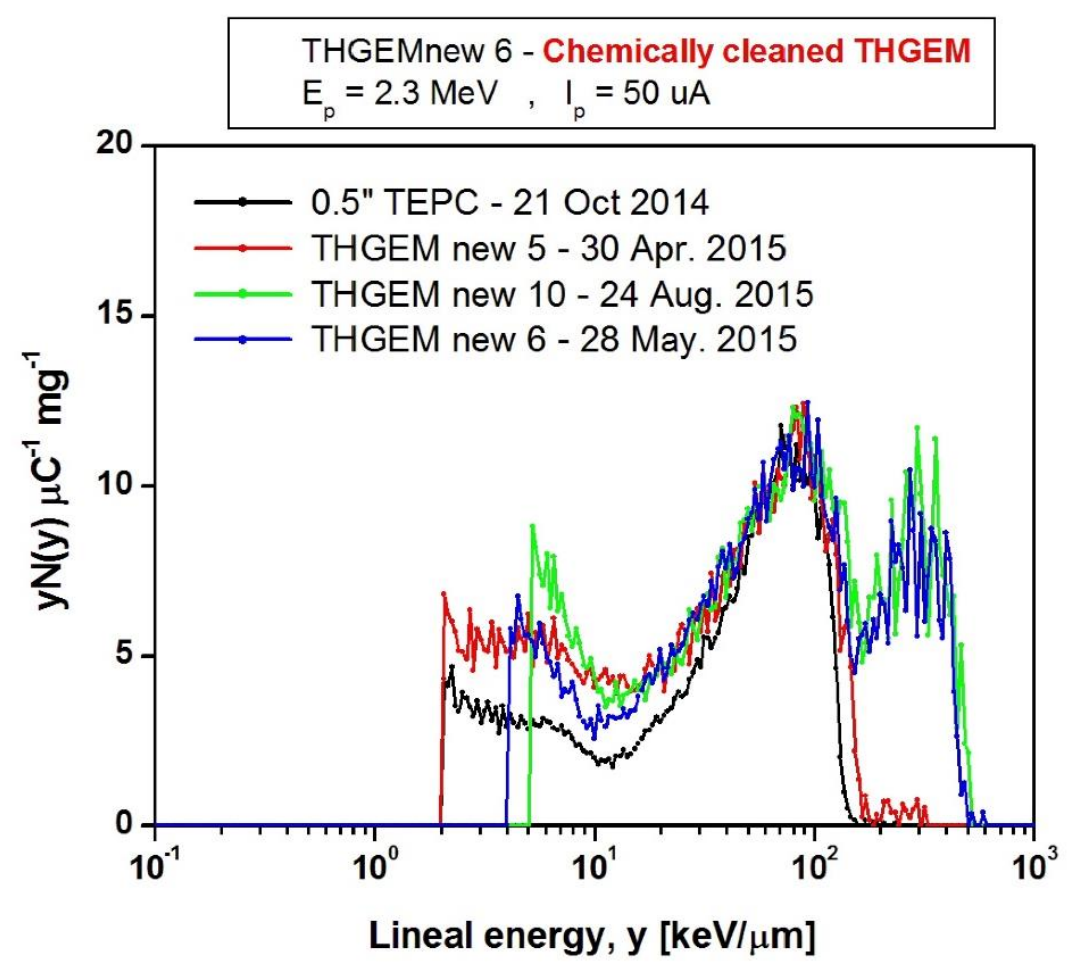

Fig. 7.16 THGEM detector microdosimetric spectrum for the chemically cleaned THGEM \#6 compared to the spectrum for THGEM \#5 and \#10 from the new batch and commercial 1/2" TEPC.

$\Delta \mathrm{V}_{\text {THGEM\#6 }}=883.33 \mathrm{~V}, \Delta \mathrm{V}_{\text {THGEM\#5 }}=850 \mathrm{~V}, \Delta \mathrm{V}_{\text {THGEM\#6 }}=850 \mathrm{~V}$ 


\subsubsection{Performance Test Results using prototype MSI-DSP vs. Commercial DSP}

From the results of the benchmark test using a NaI detector and gamma-ray calibration sources, previously presented in chapter 3 , the satisfactory performance of the single input DSP was verified. It was concluded that for the count rates dealt with in this thesis with the 2D THGEM detector, the performance of MSI-DSP compared to the commercial digital and analogue systems was superior and the results were satisfactory. In this section, the performance test results of the single input DSP coupled to the THGEM detector, using the Tandeton accelerator neutron source described in chapter 4 (section 4.1), will be presented. The goal was to determine the proper gain required for MSI-DSP and to investigate the performance of MSI-DSP with the THGEM detector and to compare the results to those of the commercial digital system. To this end the experimental setup, which was previously explained in chapter 4 (section 4.2.2), was employed.

Fig. 7.17 is the plot of raw spectra acquired with two different DSPs: ORTEC DSPEC and MSI-DSP. Fig. $7.17 a$ shows the spectra with 3 different DSPEC gains with 16k channels. Clearly, a gain of 20 is too high, so that all the events could not be recorded. Thus the gain was decreased to 10 and 5. In this case, all the events could be recorded within the 16k range. Fig. $7.17 b$ shows the spectrum acquired with MSI-DSP with a fixed gain of 10 with $2 \mathrm{k}$ channels. The direct comparison of raw data is not possible in this case as the data acquired with DSPEC was over 16k channels unlike for the MSI-DSP over 2k channels. However, the acquired data can be compared after lineal energy calibration. The microdosimetric spectra for both systems obtained with a proton energy of $2.3 \mathrm{MeV}$ and current of $100 \mu \mathrm{A}$ are illustrated in Fig. 7.18. 


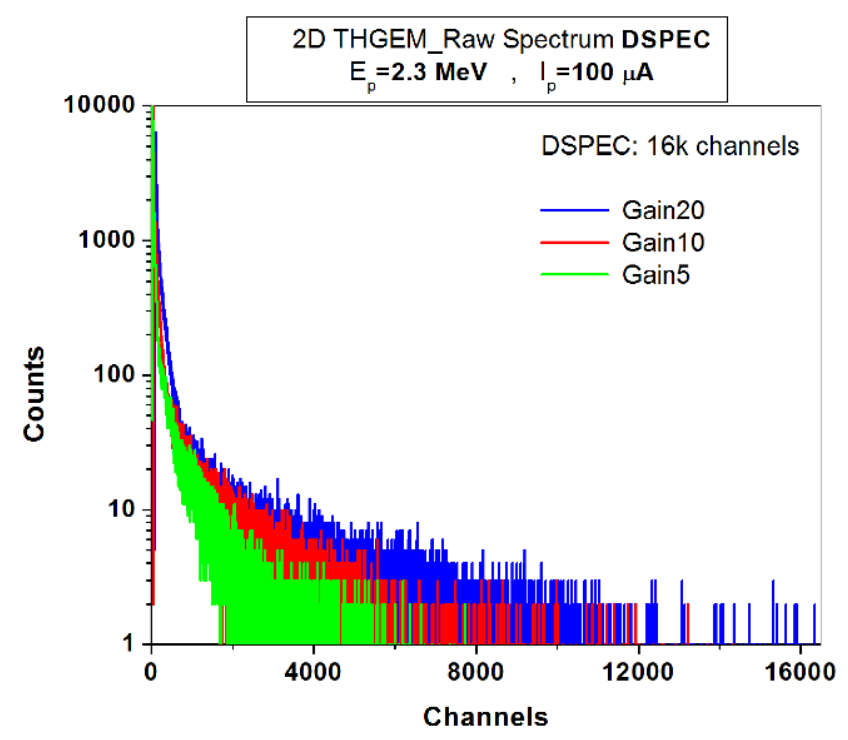

a.

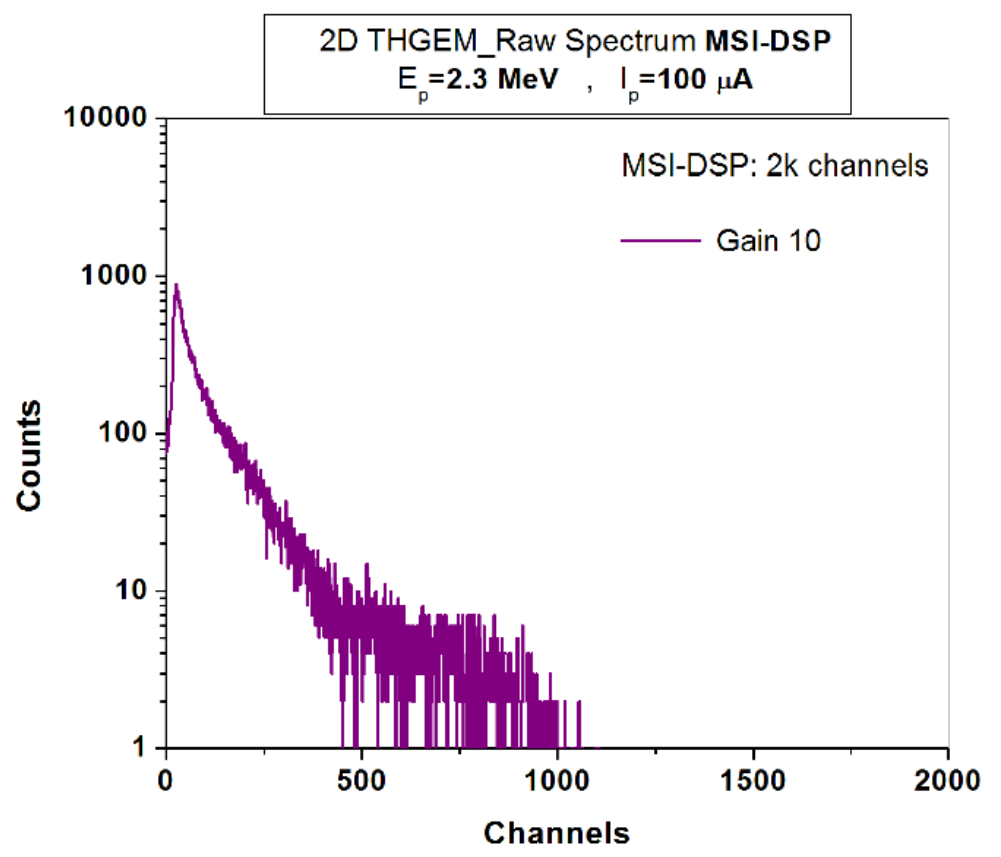

b.

Fig. 7.17 THGEM detector raw spectrum using a. DSPEC with three different gain settings, b. MSI-DSP with fixed gain of 10 


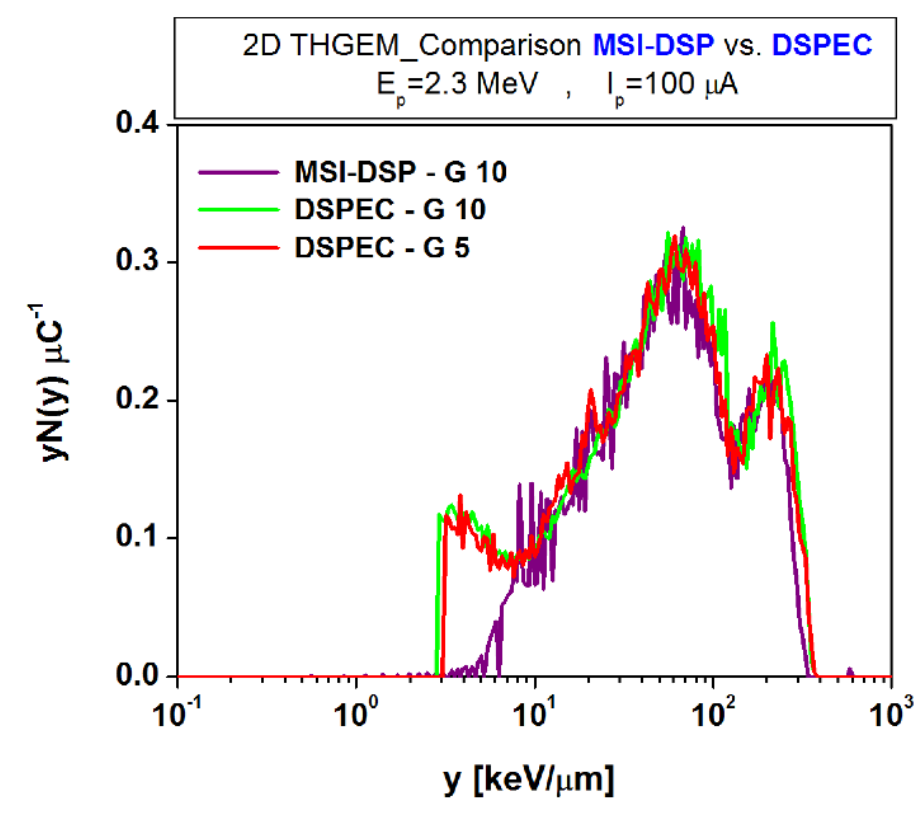

Fig. 7.18 THGEM detector microdosimetry spectra: comparison between DSPEC and MSI-DSP

From this Fig. it is obvious that the patterns of the microdosimetric spectra are consistent for the two systems. The low lineal energy part of the spectrum of the MSI-DSP below $10 \mathrm{keV} / \mu \mathrm{m}$ shows a noticeable deviation from the DSPEC. This difference results from the fact that the LLD was defined much higher above the noise level for MSI-DSP. Also, the spectrum obtained from MSI-DSP is shakier and the statistics are poorer. This is due to the analysis procedure for $2 \mathrm{k}$ channels. When the raw data over the $2 \mathrm{k}$ channels were converted to the lineal energy, redistributed in 60 logarithmic bins, the number of data in each bin is smaller for the lower values of lineal energy. This issue will be discussed more in the next section.

\subsubsection{2k Data vs. 16k data}

To investigate the redistribution of the data into 60 bin for the ADC $2 \mathrm{k}$ channels and 16k channels systematically, the commercial 2" TEPC was employed. The experiment was carried out with both ORTEC DSPEC and MSI-DSP. The microdosimetric spectra acquired with DSPEC are shown in Fig. 7.19. The raw data was acquired with $2 \mathrm{k}$ and $16 \mathrm{k}$ ADC channels. The raw data were analyzed and converted to lineal energy according to 
the method that was explained in section 5.1. Each spectrum was redistributed into equal logarithmic bins with a resolution of 60 bins per decade for both cases of $2 \mathrm{k}$ and $16 \mathrm{k}$ channels such that the $i^{\text {th }}$ value of $y$ is:

$$
y_{0} 10^{-0.5 / 60}<y_{i}=y_{0} 10^{i / 60}<y_{0} 10^{0.5 / 60}
$$

where $y_{0}$ is the minimum redistributed $y$ value and the maximum would be:

$$
y_{\max }=y_{\min } 10^{N}
$$

where $N$ is the number of decades.

As is apparent in Figs. 19 and 20, the spectra are noticeably shakier for 2k data, particularly at the lower lineal energy regions. As was explained before, this is due to the fact that not enough data points fall into the specific bin for $2 \mathrm{k}$ channels compared to $16 \mathrm{k}$ for 60 number of bins.

To show this numerically, using the proper pulser calibration of the 2" TEPC measurement, the channel boundaries corresponding to each redistributed $y$ value were calculated. For instance, for 3 different $y$ values of 3,5 and $90 \mathrm{keV} / \mu \mathrm{m}$, the channel boundaries were presented in Table 7-2. Evidently, the number of channels fell into the bin corresponding to the lineal energy value of 3 , are 17 for $16 \mathrm{k}$ channels compared to 2 for $2 \mathrm{k}$ channels in DSPEC. For the higher values of lineal energy (e.g. $90 \mathrm{keV} / \mu \mathrm{m}$ ), the interval is much bigger and there are more channels and counts in the bin and therefore the spectrum is much smoother at the proton peak region.

\begin{tabular}{|c|c|c|c|c|}
\hline \multirow[b]{2}{*}{$\mathrm{keV} / \mu \mathrm{m}$} & \multirow[b]{2}{*}{ Channel } & \multicolumn{2}{|c|}{ DSPEC } & \multirow{2}{*}{$\begin{array}{l}\text { MSI-DSP } \\
\text { 2k } 60 \mathrm{Bin} \\
\end{array}$} \\
\hline & & $16 k-60$ Bin & $2 k-60 \mathrm{Bin}$ & \\
\hline \multirow{3}{*}{$y=3$} & $\mathrm{Ch}_{\min }$ & 180 & 23 & 29 \\
\hline & $\mathrm{Ch}_{\max }$ & 197 & 25 & 33 \\
\hline & \# of ch & 17 & 2 & 4 \\
\hline \multirow{3}{*}{$y=5$} & $\mathrm{Ch}_{\min }$ & 278 & 35 & 50 \\
\hline & $\mathrm{Ch}_{\max }$ & 306 & 39 & 56 \\
\hline & \# of ch & 28 & 4 & 6 \\
\hline \multirow{3}{*}{$y=90$} & $\mathrm{Ch}_{\min }$ & 4427 & 569 & 942 \\
\hline & $\mathrm{Ch}_{\max }$ & 4933 & 634 & 1051 \\
\hline & \# of ch & 506 & 65 & 109 \\
\hline
\end{tabular}

Table 7-2. Comparison of number of channels that fall into a logarithmic bin for $16 \mathrm{k}$ and $2 \mathrm{k}$ ADC channels 


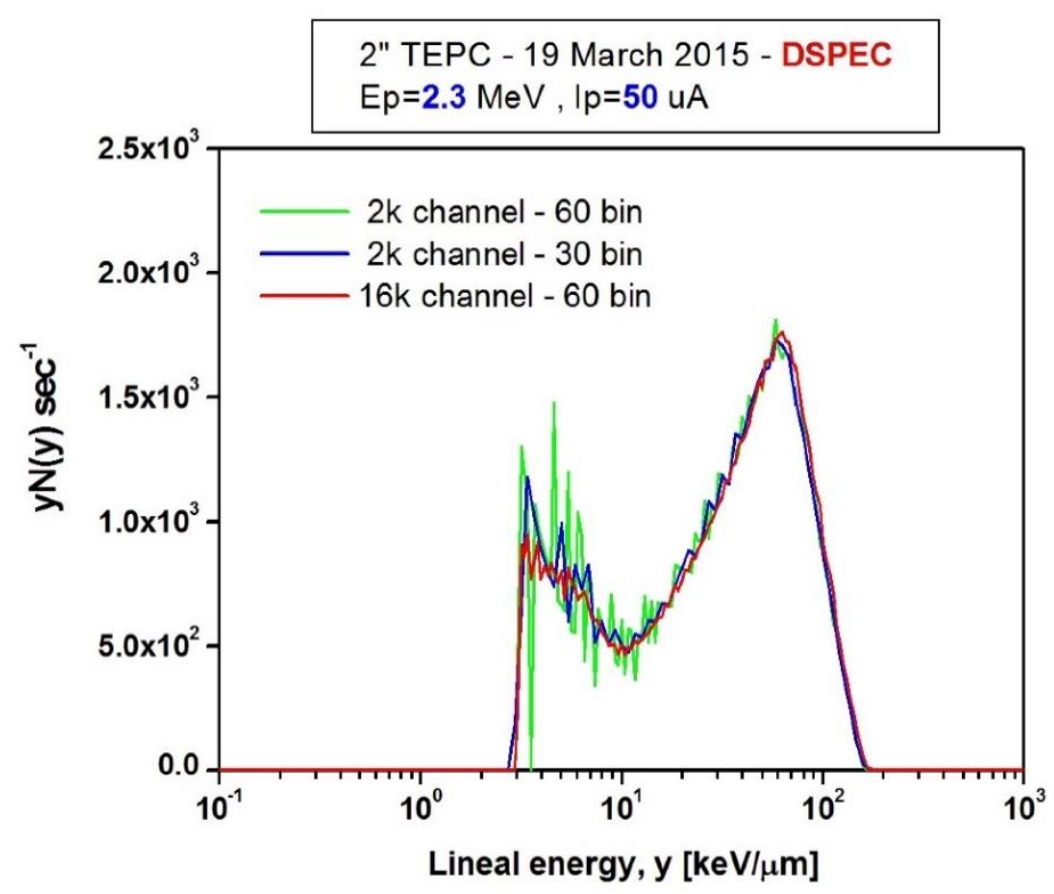

Fig. 7.19 2" TEPC microdosimetry spectra: comparison for DSPEC between $2 \mathrm{k}$ and $16 \mathrm{k}$ channel

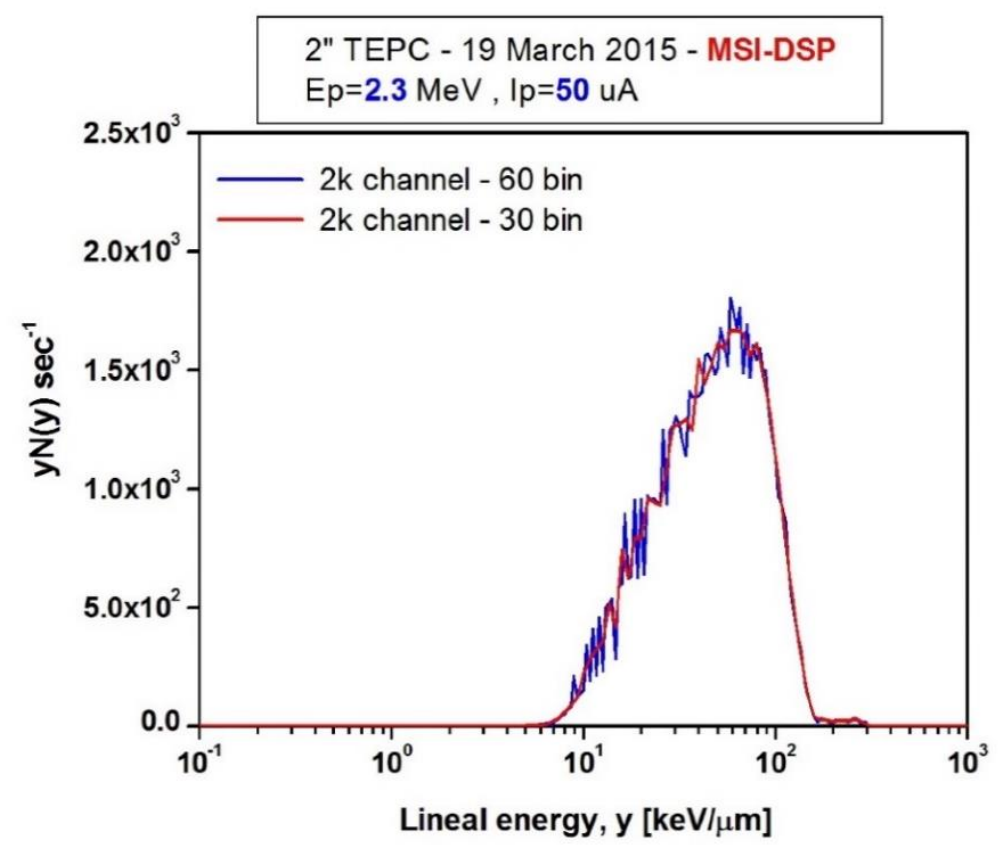

Fig. 7.20 2" TEPC microdosimetry spectra: comparison for MSI-DSP between 60 and 30 bin number of bins 
In order to show to have smoother spectra, the number of bins was reduced to 30 , and the results are compared in Fig. 7.19 and 7.20 for DSPEC and MMI-DSP, respectively. Obviously, the data for 30 bins were much smoother. Generally, a higher number of bins leads to a more accurate distribution, but less number of events into a specific bin. To keep the data analysis consistent with the previous studies carried out in this group, 60 number of bins were used for the entire analyses in this thesis.

\subsubsection{Summary}

From the results presented in this chapter it is evident that even though the THGEM detector size is about 2.54 times smaller than the 1/2" TEPC and about 11.38 times smaller than the 2" TEPC, whose efficiencies are much higher for a given time, the THGEM detector showed a consistent microdosimetric pattern and acceptable statistical fluctuations.

Likewise, from these results, the ability to confidently employ the McMaster custom made signal processing system with the 2D THGEM detector was ascertained. Thus the prototype single channel DSP design was miniaturized to devise 5 DSPs on a single board for the multi-input application (see section 3.2) which will be presented in the next section. 


\subsection{D Multi-volume Detector $(3 \times 3$ gaseous Cavity Array)}

In all the measurements carried out with the $3 \times 3$ gaseous cavity array, THGEMs from the new batch were used. Using the experimental setup explained in chapter 4 (section 4.2.3), the $2 \mathrm{D}$ multi-volume detector response was investigated. The map of the detectors inside the chamber is shown in Fig. 7.21. Detector 5 is the central detector, which was tested in the previous section as a single volume detector. Detectors 1, 3, 7 and 9 are located at the corners and detectors 2, 4, 6 and 8 are located at the sides. The distance between each pair of detectors on the side is $2.25 \mathrm{~cm}$.

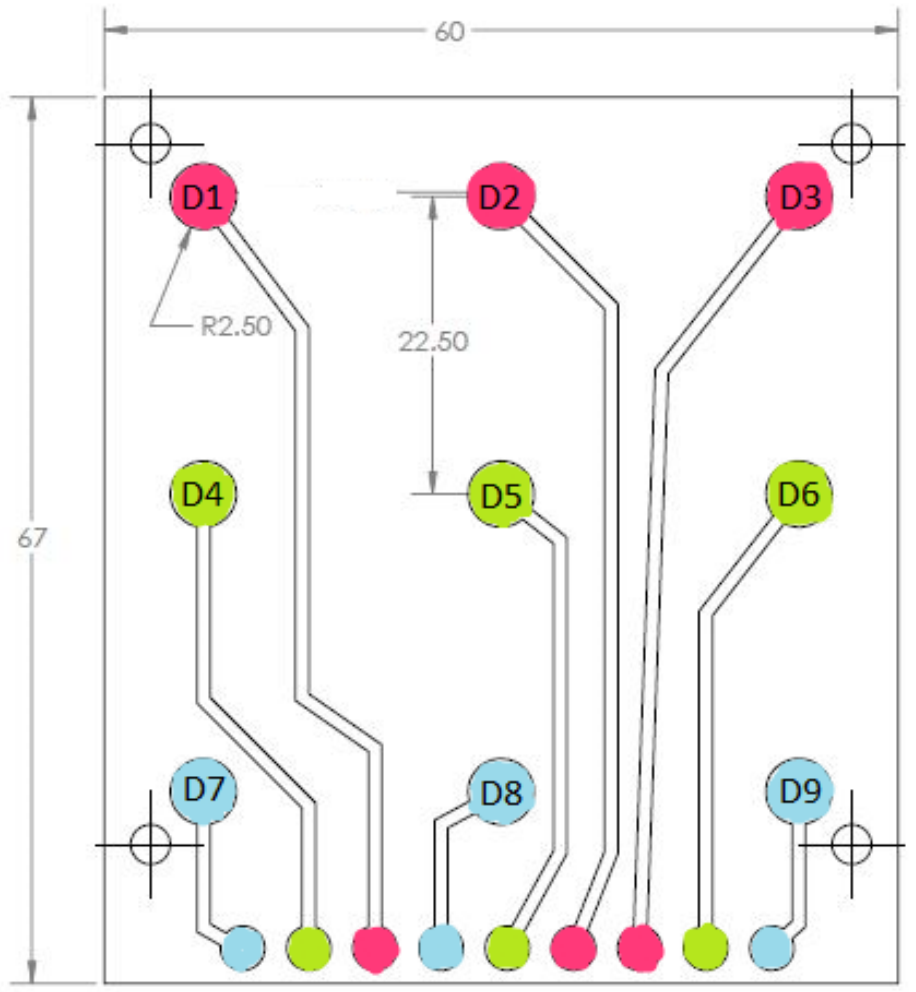

Fig. 7.21 Array of $3 \times 3$ detectors: 9 individual detectors numbered accordingly for convenience

The detectors response were evaluated using both the single-layer and double-layer THGEM configurations (see chapter 2, section 2.7.2) and the results will be presented in the following subsections. 


\subsubsection{D Single-Layer THGEM Detector Performance}

\section{Detector assembly cables check and detector stability}

The first set of measurements was carried out using the new THGEM \#9, and the commercial ORTEC DSPEC. Each detector was tested individually using the DSPEC for data acquisition. The goal was to test the detector assembly, if the interconnection wires were soldered properly, and to check if the assembled multi-pin cable plug (connecting the multi-pin on the detector to the CAEN preamplifier inputs, see Fig. 2.13) was working. As there were many unknown features and parameters for testing the newly assembled $2 \mathrm{D}$ detector, this was done to ensure everything else was functioning without any problem.

The detector placed inside the cavity perpendicular to the neutron beam produced by proton energy and current of $2.3 \mathrm{MeV}$ and $50 \mu \mathrm{A}$ respectively, at the McMaster Tandetron accelerator facility (see section 4.2.3). With the beam on, the high voltage was applied to the cathode and the THGEM individually. By raising high voltage gradually, one of the detector's signals (usually D5) was observed carefully on the oscilloscope to find out the operating high voltage of the detector and avoid any discharge due to excess applied voltage. Once the signal grew sufficiently and detector reached its proportional region as shown in Fig. 7.22, the increasing of voltage was ceased. Based on previous experience with the THGEM, usually, this is the safest voltage that could possibly be applied.

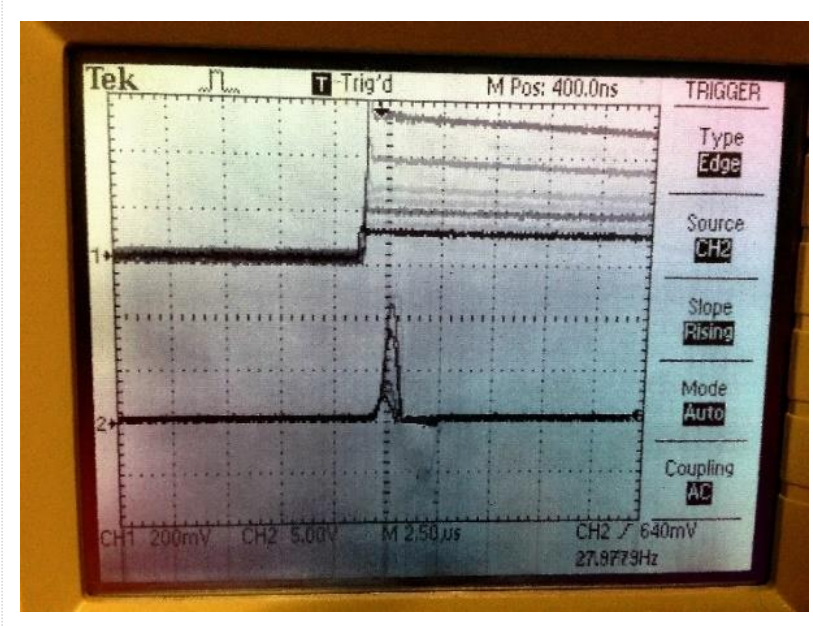

Fig. 7.22 Detector signal in proportional region 
To acquire a reasonable spectrum, the DSPEC gain was adjusted in a way that all the events could be collected within the 16k ADC channels and none of the events were exceeding this range. For this THGEM, $\mathrm{HV}_{\text {Cathode }}$ and $\mathrm{HV}_{\text {THGEM }}$ were set to $1120 \mathrm{~V}$ and $1020 \mathrm{~V}$ respectively, and the DSPEC gain was set to 1 . All the spectra for 9 detectors were collected at these settings.

Fig. 7.23 shows the gain stability for the central detector (D5) during a 20 min interval at the beginning of the measurement. It is evident that the detector gain did not drop rapidly and it was acceptably stable during this interval. To investigate the response of the detector over a longer period of time, multiple measurements with consistent settings were carried out over $2 \mathrm{~h}$ intervals. Fig. 7.24 demonstrates the raw and microdosimetric spectra for each measurement at a different time. Each spectrum was acquired for $600 \mathrm{sec}$. The spectra patterns are consistent except for the events happening after the proton edge which was discussed before. As can be seen from the raw data and microdosimetric spectra, the interesting point is that the extra events appear over time. The proton edge was estimated at channel 4700 for $\mathrm{t}=20 \mathrm{~min}, 3500$ for $\mathrm{t}=2 \mathrm{~h}$ and 3200 for $\mathrm{t}=4 \mathrm{~h}$, meaning that over time the gain dropped and at the same time higher lineal energy events appeared. As was shown previously and with the standard 1/2" TEPC, these events cannot happen at these range of neutron energies physically, so there should be another systematic issue that causes these extra events. Also, the lower end lineal energy cut off of the spectra was increased. This may be due to the fact that over time, the detector gain dropped. Many attempts were made, which will be presented through this section, to address this issue. 


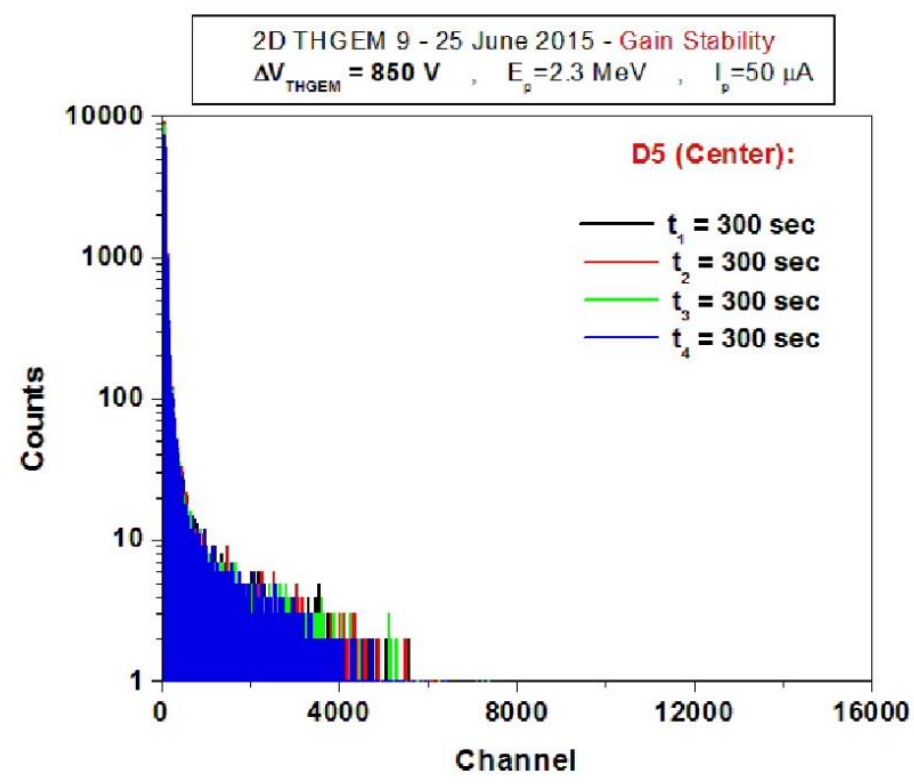

a.

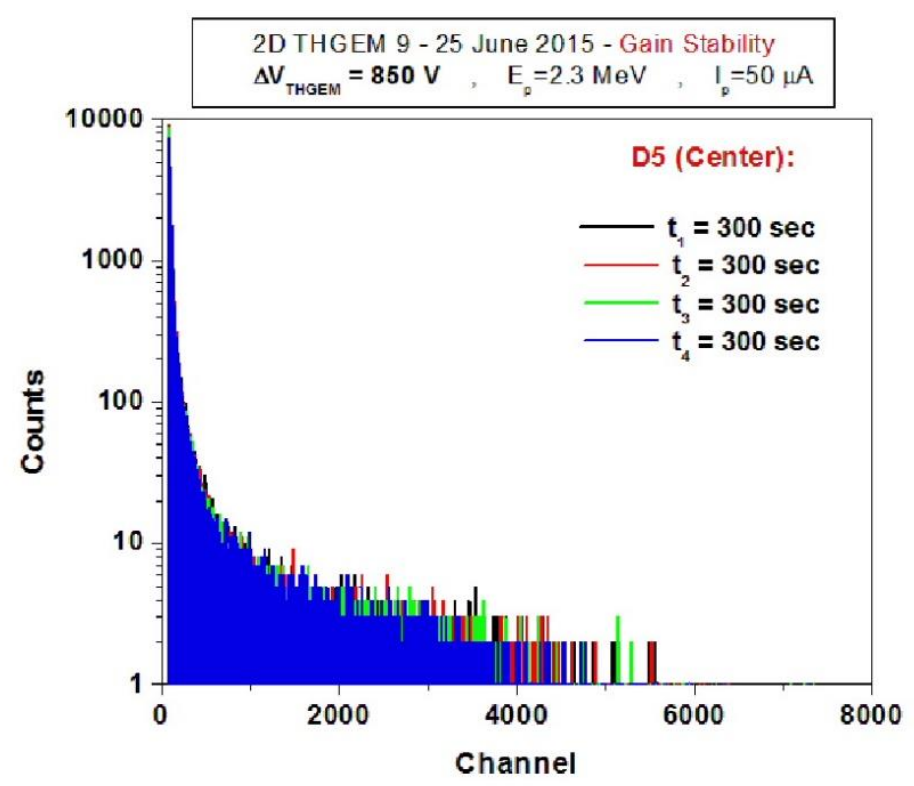

b.

Fig. 7.23 THGEM detector (D5) raw spectrum using DSPEC and CAEN preamplifier a. full scale b. half-scale 


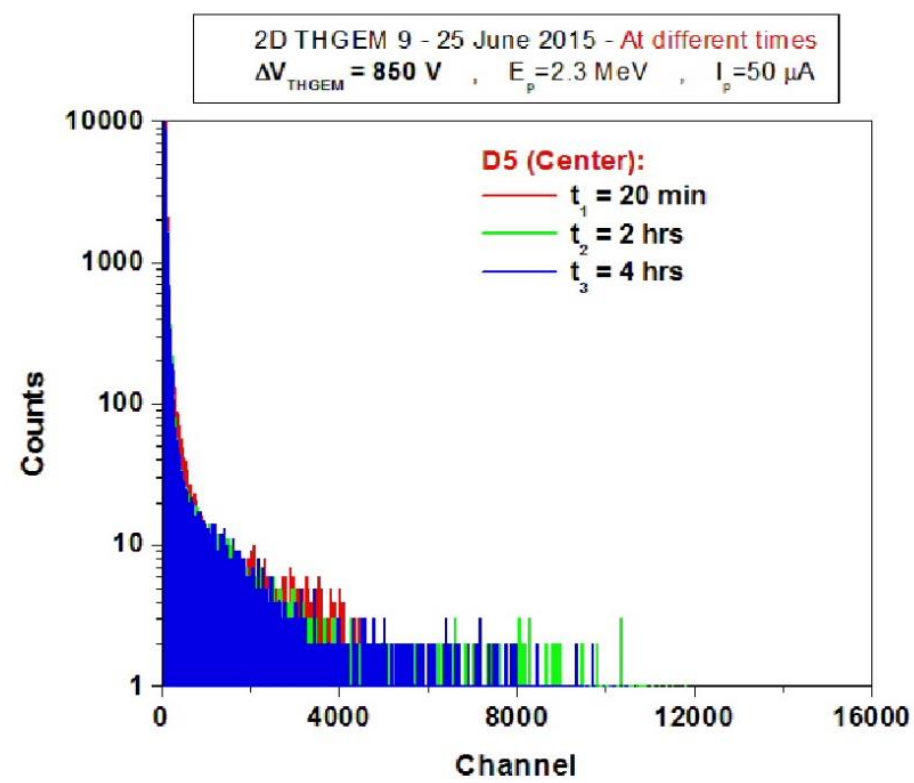

a.

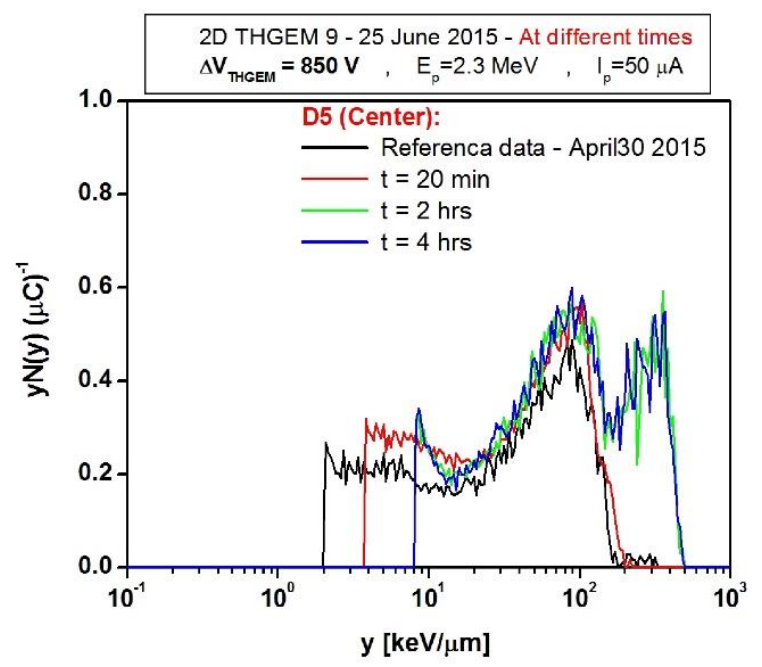

b.

Fig. 7.24 THGEM detector (D5) spectrum acquired using DSPEC and ORTEC preamplifier at different times for 10 min each a. Raw data, b. microdosimetric spectra 
Fig. 7.25 shows the raw spectrum for all the other detectors collected individually with $\mathrm{E}_{\mathrm{p}}=2.3 \mathrm{MeV}$ and $\mathrm{I}_{\mathrm{p}}=50 \mu \mathrm{A}$ for $10 \mathrm{~min}$ real time. The live time was $598 \mathrm{~s}$ and the dead time was around $0.33 \%$. The count rates for all 9 detectors for $\mathrm{E}_{\mathrm{p}}=2.3 \mathrm{MeV}$ are reported in Table 7-3. As the detectors are located at different positions the count rates vary from the maximum for the central detector (D5) and lower for the side and corner ones as expected.

Table 7-3. Detectors (THGEM \#9) count rate for $\mathrm{E}_{\mathrm{p}}=2.3 \mathrm{MeV}$ and $\mathrm{I}_{\mathrm{p}}=50 \mu \mathrm{A}$

\begin{tabular}{llllllllll}
\hline Detector & D1 & D2 & D3 & D4 & D5 & D6 & D7 & D8 & D9 \\
\hline CR [cps] & 167 & 610 & 221 & 487 & 612 & 604 & 478 & 153 & 336 \\
\hline
\end{tabular}

The response of the detectors was examined for different proton energies as shown in table 7-4. As expected, by increasing the proton energy from $1.8 \mathrm{MeV}$ to $2.5 \mathrm{MeV}$, the count rate increased as well.

Table 7-4. Central detector (D5) count rate for various proton energies and $\mathrm{I}_{\mathrm{p}}=50 \mu \mathrm{A}$

\begin{tabular}{ccccc}
\hline $\mathbf{E}_{\mathrm{p}}[\mathrm{MeV}]$ & $\mathbf{1 . 8}$ & $\mathbf{2}$ & $\mathbf{2 . 3}$ & $\mathbf{2 . 5}$ \\
\hline $\mathrm{CR}_{\mathrm{D5}}[\mathrm{cps}]$ & 6 & 124 & 620 & 1015 \\
\hline
\end{tabular}

Fig. 17.26 shows the microdosimetric spectrum for all the 9 detectors compared to the central detector (D5). From this Fig. it can be seen that the proton peak height is much smaller in D1 compared to D5, however, it is higher in D8. Comparing their spectra, it seems that the neutron fluence on the right side of the center is slightly higher than the left side, as the proton peak height in D3, D6 and D9 is slightly larger than in D1, D4 and D7. This was a promising result for the preliminary experiment, as all the 8 sides and corners detectors had a reasonable signal and their microdosimetric spectra follow the same pattern as the standard TEPC.

With these results, the functionality of the multi-pin, cables, multi-pin plug and electronics was successfully ascertained. However, more measurements had to be carried out to address the discrepancy that appeared in D1 and D8 and to investigate the reproducibility of the results. In the following, the results of these measurements will be presented and discussed in more detail. 


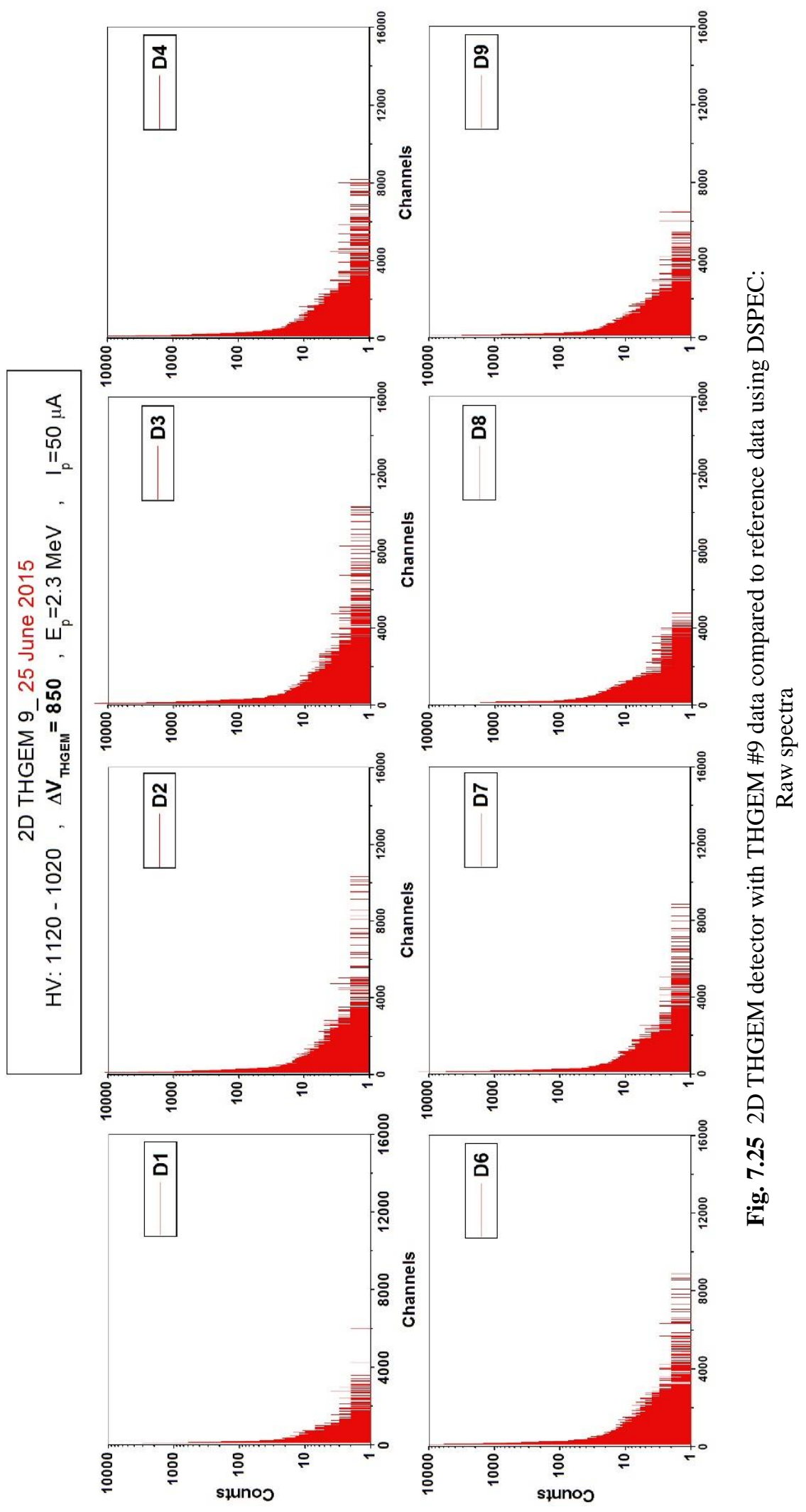




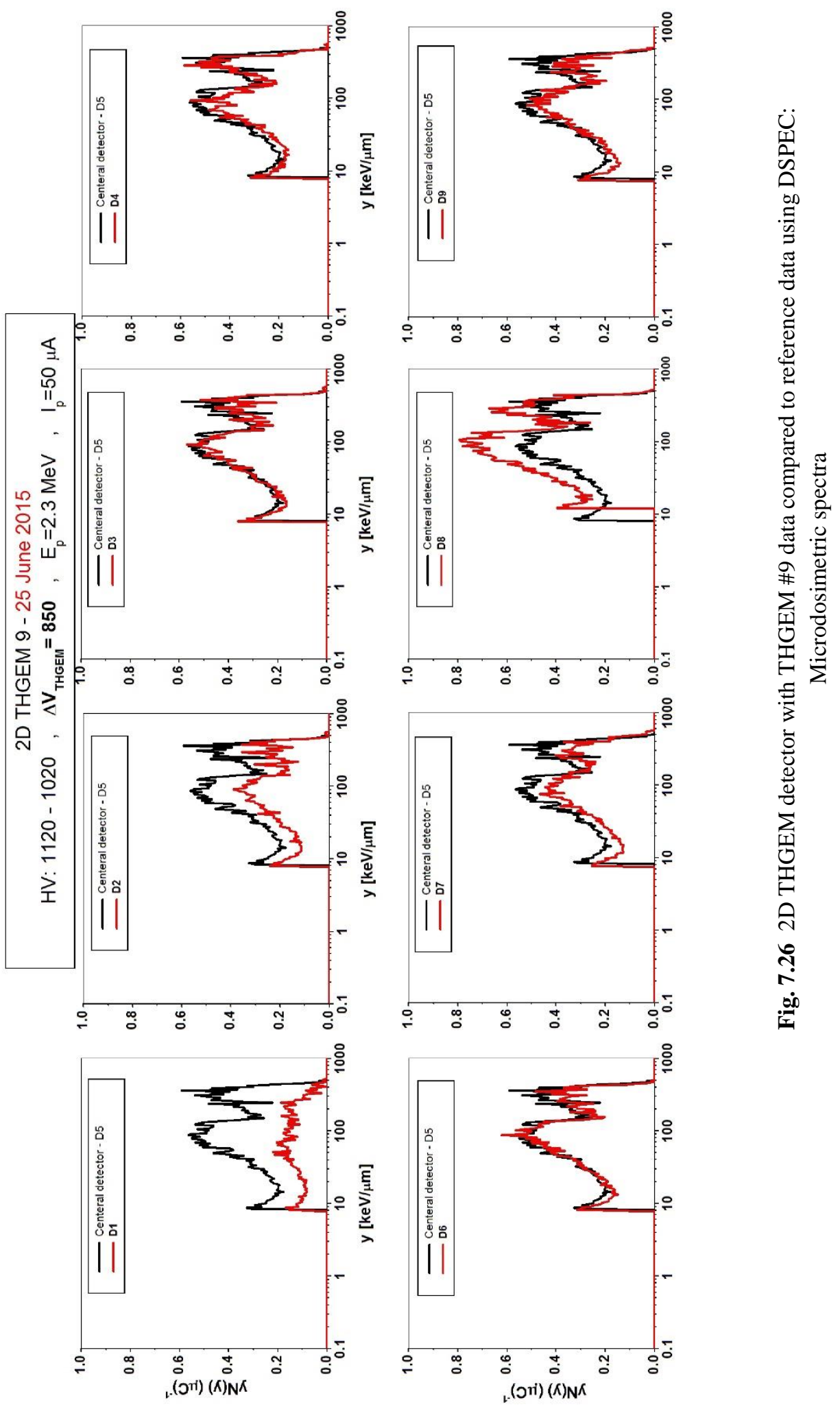




\section{D THGEM Detector Test using MMI-DSP}

Next, all the 9 detectors responses were investigated simultaneously using the custom made MMI-DSP. The same settings, such as Cathode and THGEM high voltages, detector position, acquisition time, proton energy and current, were utilized and the results were compared to the ones acquired with the commercial DSPEC in the first measurement.

It is worth mentioning the order of detectors connection to the MMI-DSP. As is depicted in Fig. 7.27, there are 9 detectors and 10 channels of MMI-DSP (5 DSPs allocated on each board). The detectors and channels were numbered and labelled accordingly. There was one channel extra for this number of detectors and it was left spare in case of failure in any other channel.

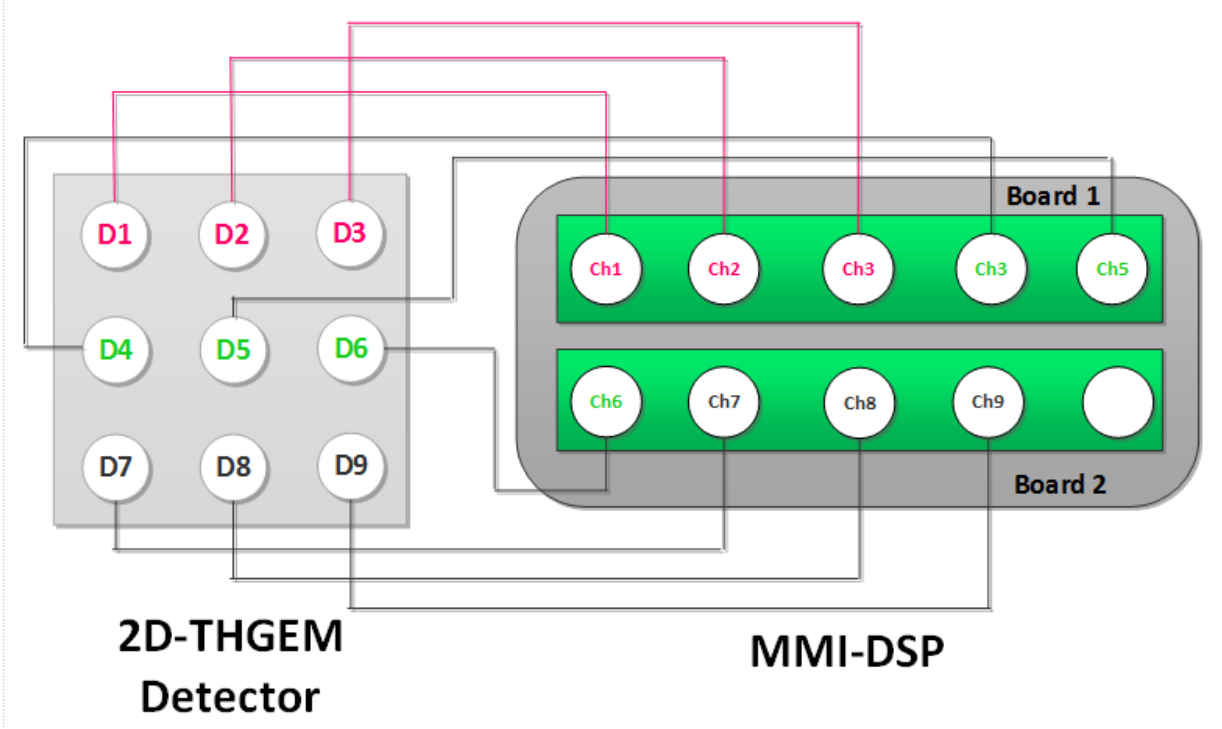

Fig. 7.27 The order of detectors connections to the MMI-DSP. The array of $3 \times 3$ detector: 9 individual detectors numbered and connected to the channels of MMI-DSP accordingly.

From the very beginning using MMI-DSP, it was found that channel \#3 (hereafter Ch3) was not working and the spectrum of D3 could not be acquired through it. The Spectrum for each detector together with the pulser calibration is shown in Fig. 7.28. Note that the pulser test input on the single unit preamp was not working for this particular measurement. So the pulser data was missing for D9 in this Fig. This issue was resolved afterwards. 

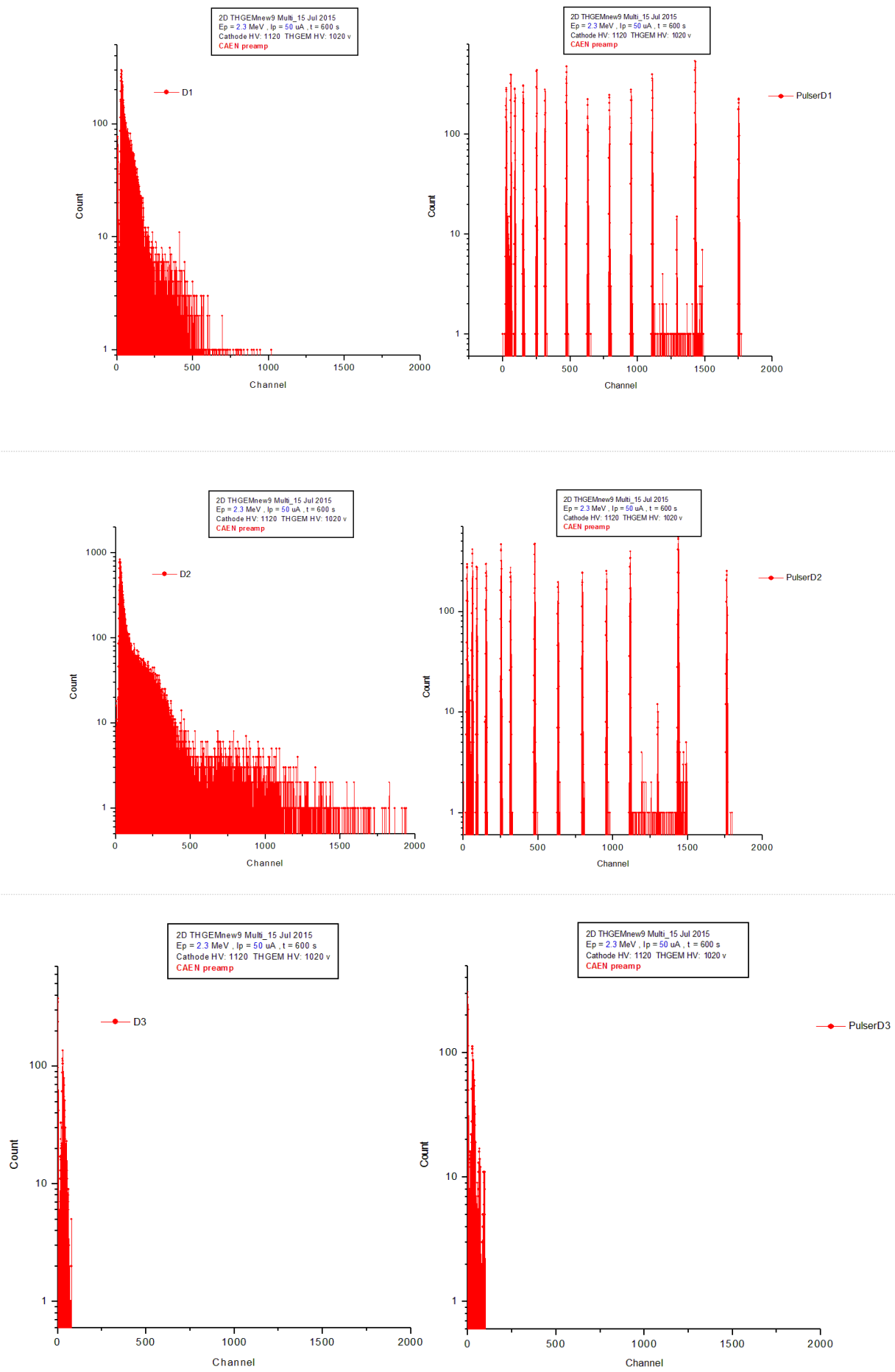

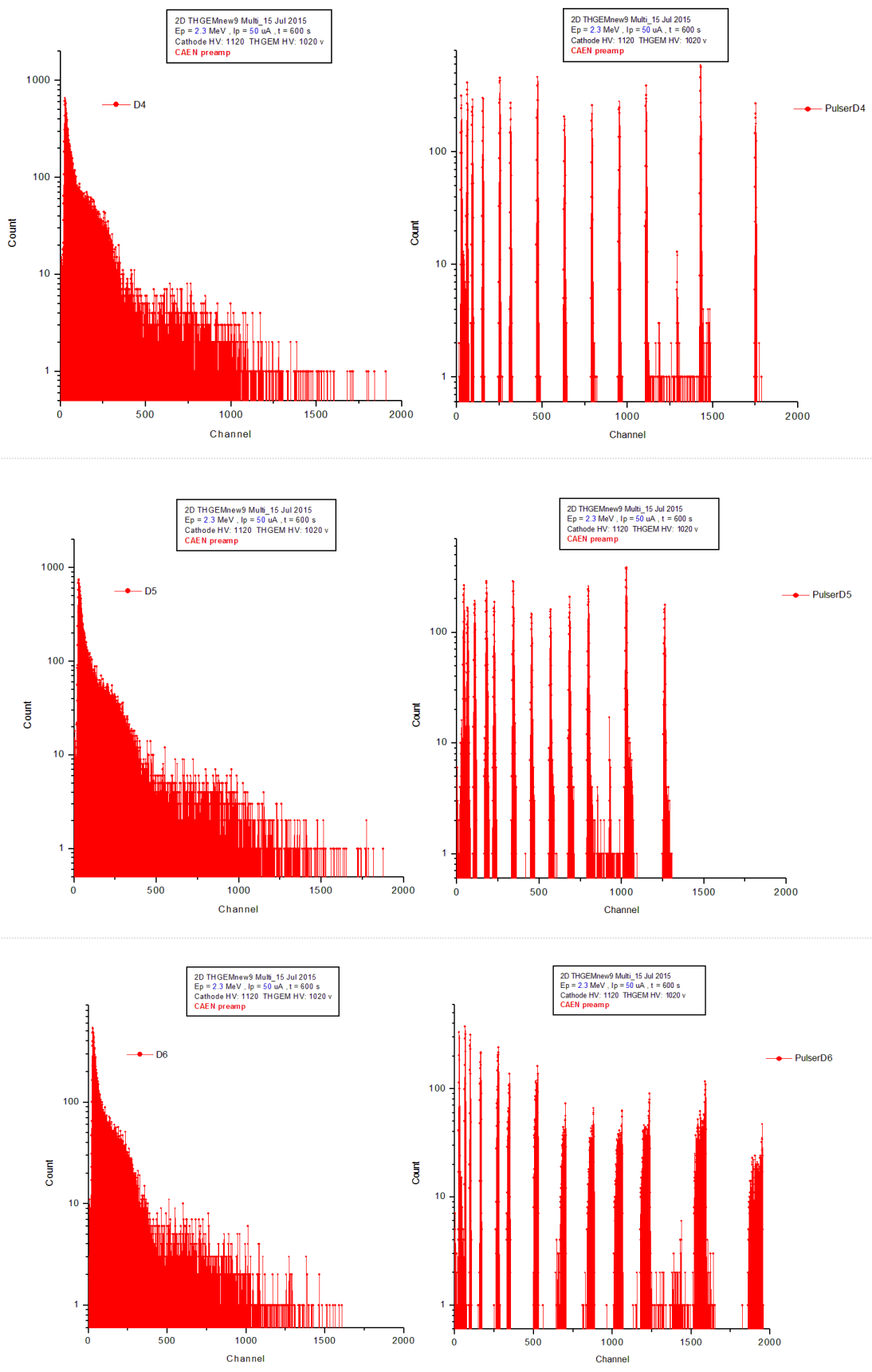

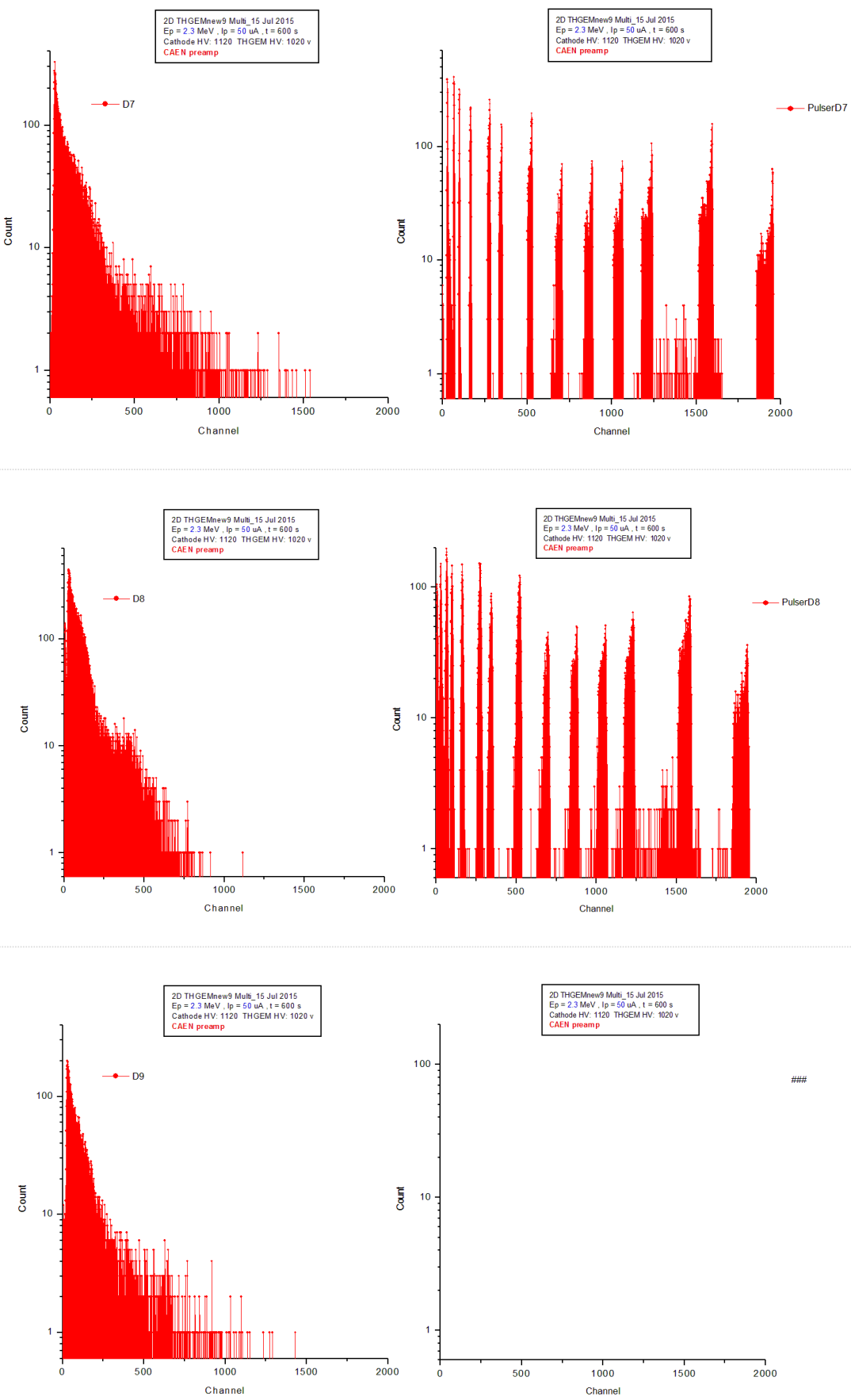

Fig. 7.28 The 2D THGEM detector raw spectrum using the MMI-DSP $\left(E_{p}=2.3 \mathrm{MeV}\right.$ and $\left.\mathrm{I}_{\mathrm{p}}=50 \mu \mathrm{A}\right)$. The plot on the left side in each row shows the raw spectrum of each detector and the plot on the right side is the corresponding pulser calibration for that detector and MMI-DSP input a. D1, b. D2, c. D3, d. D4, e. D5, f. D6, g. D7, h. D8, I. D9 
To check if this was the Ch3 functionality failure or D3 did not have any signal itself, the detectors were connected to the MMI-DSP according to Fig. 7.27 and D3 was directly connected to ORTEC DESPEC. Proton energy and current again were set to $2.3 \mathrm{MeV}$ and $50 \mu \mathrm{A}$. This time, a pulser was turned on and the amplitude was set such that the pulse appeared above channel 1500 with $60 \mathrm{~Hz}$ frequency. Fig. 7.29 depicts the raw spectrum of each detector and their corresponding pulser calibration. From Fig. $7.29 c$ it is evident that D3 has a signal and spectrum. Therefore the previous issue was not from the detector but from the $\mathrm{Ch} 3$ of the MMI-DSP.

From this, the pulser peak position, count rate and resolution were analyzed to compare each channel of the MMI-DSP. These results are presented in table 7-5. Except for D8 which was connected to ch8, the rest of the detectors connected to their corresponding DSP input were not significantly different. The pulser peak resolution in D8 was double than the other detectors and inputs.

Table 7-5. Detectors (THGEM \#9) count rate for $E_{p}=2.3 \mathrm{MeV}$ and $I_{p}=50 \mu A$

\begin{tabular}{cccccccccc}
\hline Detector & D1 & D2 & D3 & D4 & D5 & D6 & D7 & D8 & D9 \\
\hline MMI-DSP & ch1 & ch2 & DSPEC & ch4 & ch5 & ch6 & ch7 & ch8 & ch9 \\
\hline $\begin{array}{c}\text { Peak } \\
\text { Position } \\
\text { [ch] }\end{array}$ & 1750 & 1754 & 1255 & 1748 & 1835 & 1762 & 1764 & 1780 & 1816 \\
\hline $\begin{array}{c}\text { CR [cps] } \\
\text { \% }\end{array}$ & 59.40 & 59.86 & 59.93 & 59.70 & 58.60 & 58.80 & 59.96 & 55.55 & 58.30 \\
\hline $\begin{array}{c}\text { Resolution } \\
\text { ( }\end{array}$ & 0.228 & 0.271 & 0.267 & 0.255 & 0.230 & 0.207 & 0.247 & 0.489 & 0.228 \\
\hline
\end{tabular}

After extensive hardware and systematic check, it was revealed that there is an issue in the MATLAB programming code which was resolved afterwards. Also, it was realized that the DSP 9 program's name should not be changed just by the file name, as there are many commands that are fixed within the code that use DSP 9. Therefore, when it is necessary, the best option is to just copy and paste the DSP 9 file in another folder and change the name of the folder instead. This prevents many errors in running the program. Another issue was that the USB cable should not have been disconnected when the MATLAB 
program was running. Also, it was always required to click the start, stop and clear button and restart the MATLAB once at the beginning of each measurement.
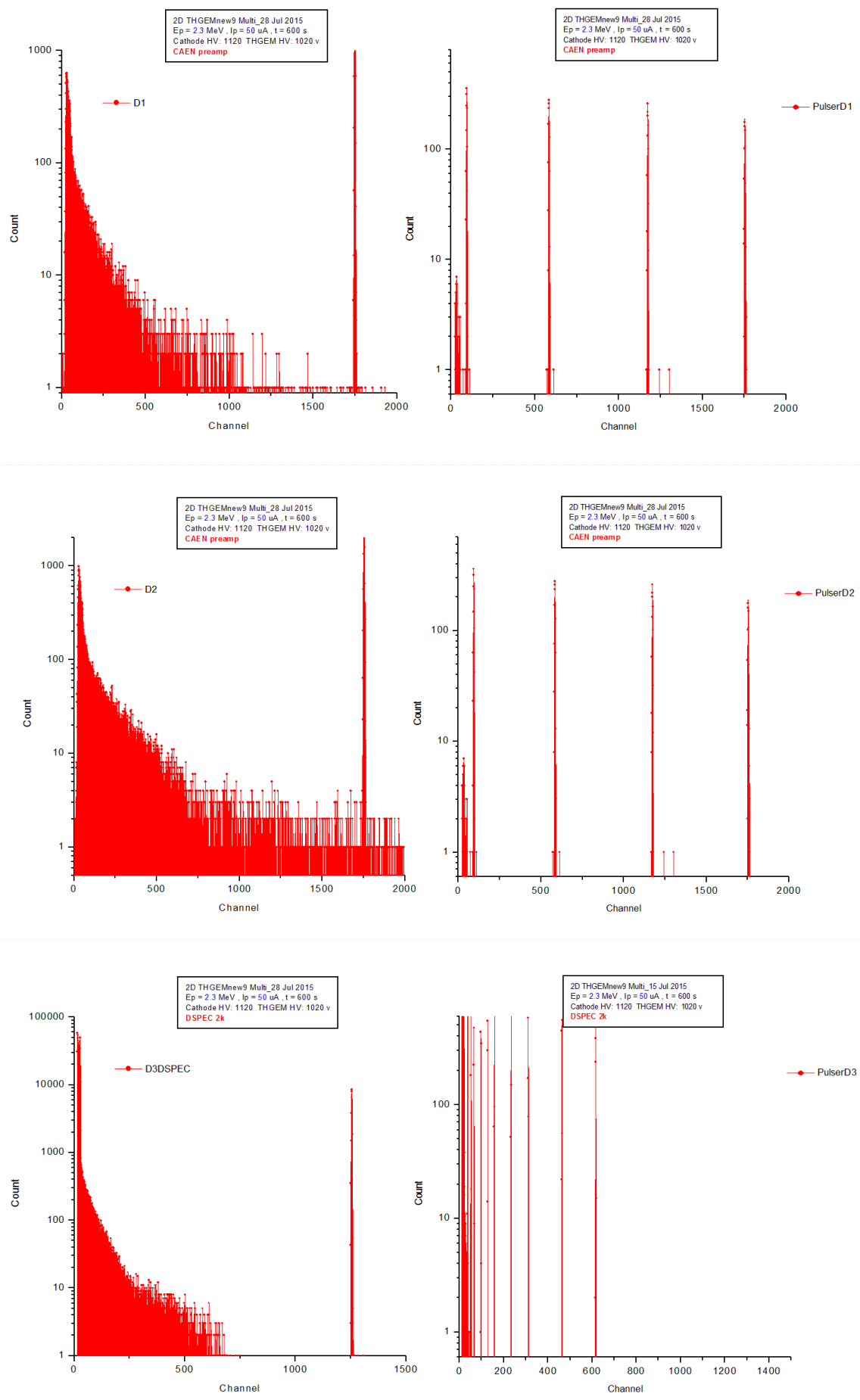

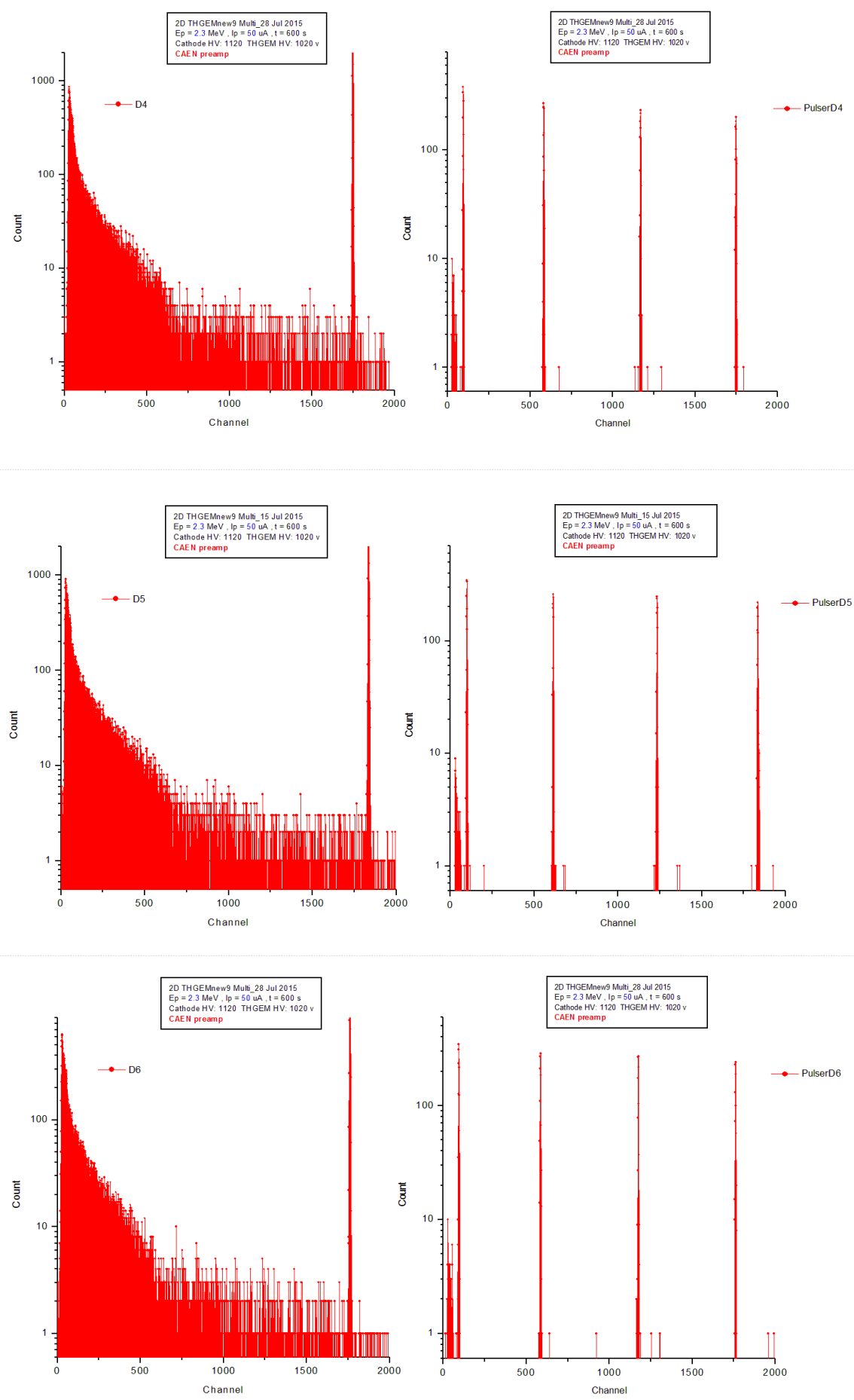

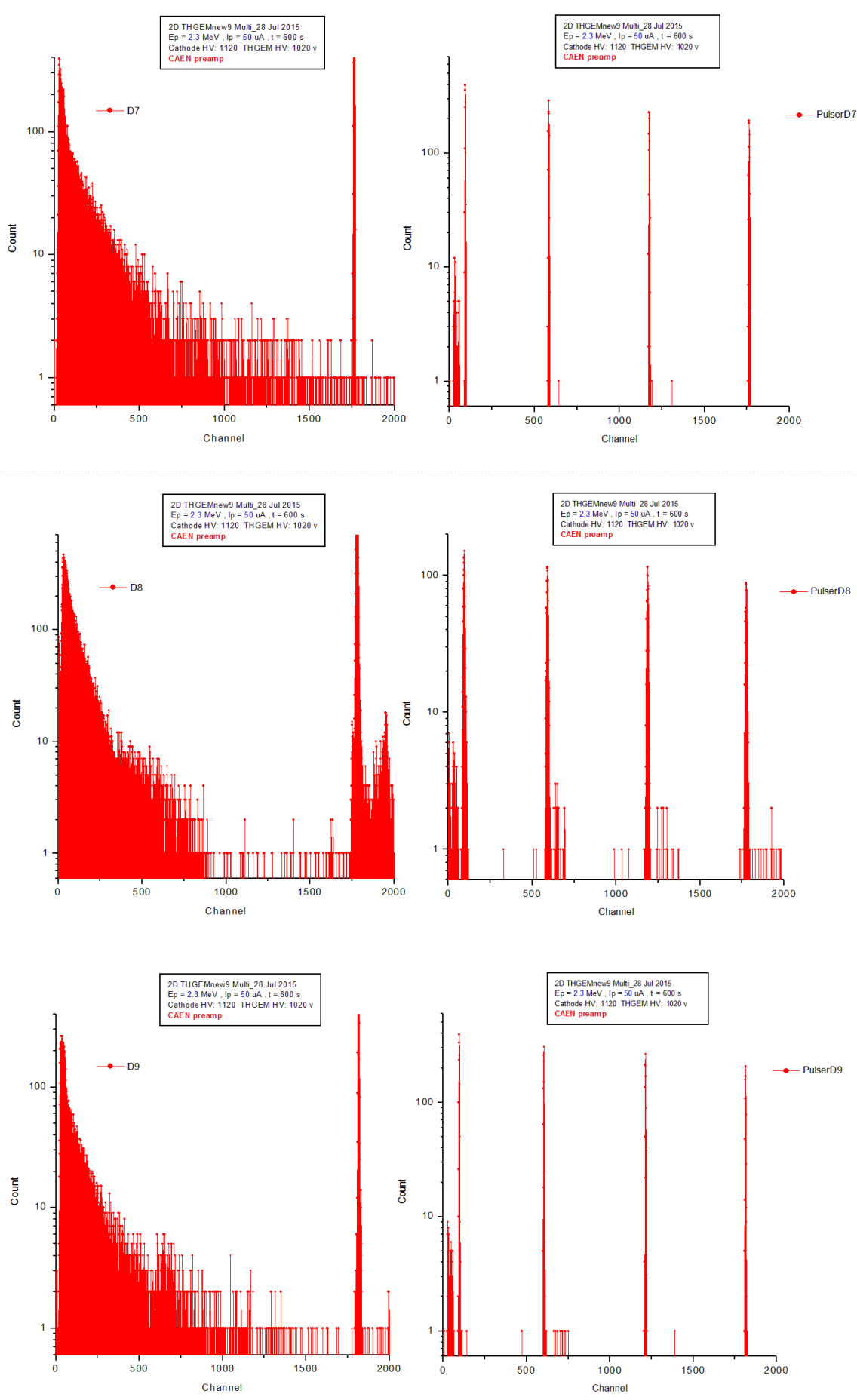

Fig. 7.29 The 2D THGEM detector raw spectrum with pulser peak using the MMI-DSP $\left(E_{p}=2.3 \mathrm{MeV}\right.$ and $\mathrm{I}_{\mathrm{p}}=50 \mu \mathrm{A}$ ). a. D1, b. D2, c. D3, d. D4, e. D5, f. D6, g. D7, h. D8, I. D9 


\section{MMI-DSP Boards Assessment}

To test if there are any differences between the MMI-DSP channels on board 1 and 2, a measurement was carried out connecting D5 to Ch1 (first channel on board 1), Ch6 (first channel on board 2) and to the DSPEC, individually. Fig. 7.30 shows the raw and microdosimetric spectra for D5. As can be seen, the central detector had the same pattern in all the three cases, however, the data acquired with ch1 had a lower height proton peak compared to the other two. Thus it was concluded that the issue was not related to the D1, but to the first input of the MMI-DSP.

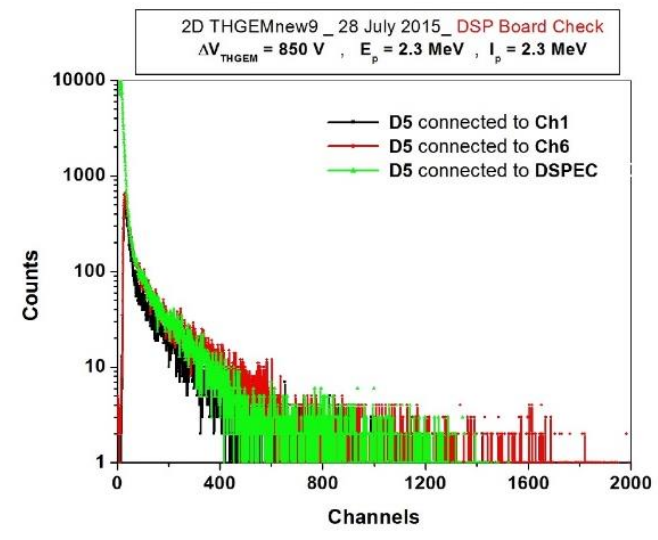

a.

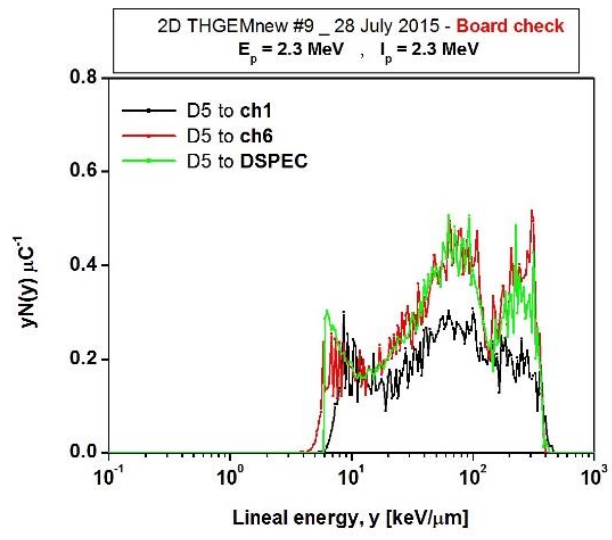

b.

Fig. 7.30 THGEM detector (D5) a. raw spectrum and b. microdosimetric spectrum, acquired using Ch1 and $\mathrm{Ch} 6$ on the MMI-DSP and DSPEC individually

To double check the reproducibility of this issue with ch1, D1 was connected to ch1 and ch5 individually and the raw and microdosimetric spectra were compared in Fig. 7.31. It was clearly observed that the two spectra do not match and the data acquired with ch1 again had a lower height proton peak. This result also supported the previous conclusion that the issue is related to the failure of the ch1, the first input of the MMI-DSP, and not to the detector functionality. 


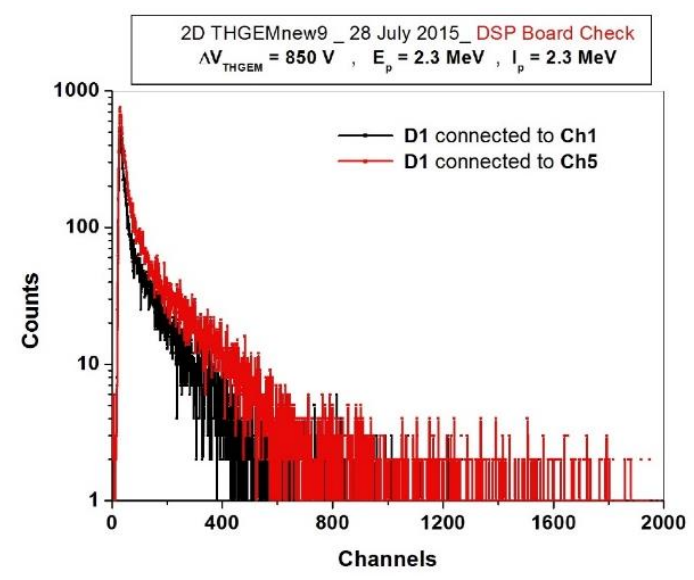

a.

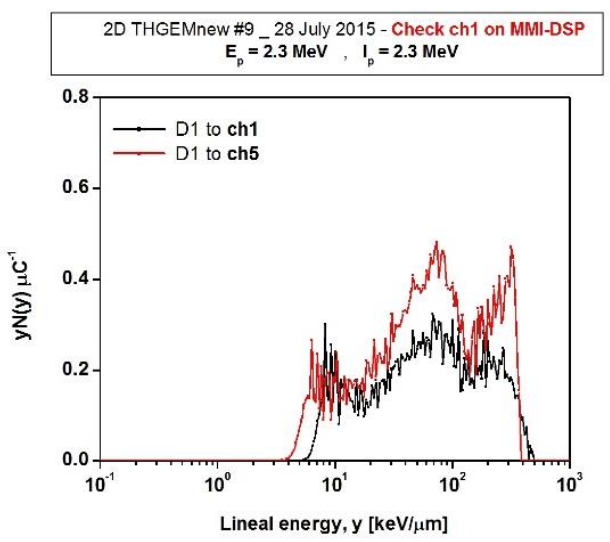

b.

Fig. 7.31 THGEM detector (D1) a. raw spectrum and b. microdosimetric spectrum, acquired using Ch1 and Ch5 on the MMI-DSP individually

Fig. 7.32 shows the raw spectra of each individual detector (apart from D5) acquired with different MMI-DSP inputs. The responses were consistent for the same detector connected to the different inputs for all the detectors except D1/ch1 as was explained above. The consistency of the results was also observed with the pulser calibration (See Fig. 7.33) which was carried out using different detectors connected to the same MMI-DSP inputs. Ch3 had already been identified as faulty, as noted previously.

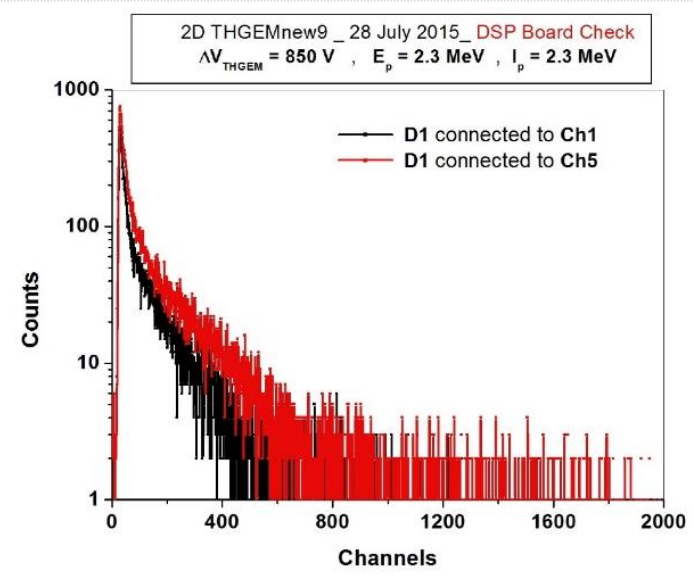

a.

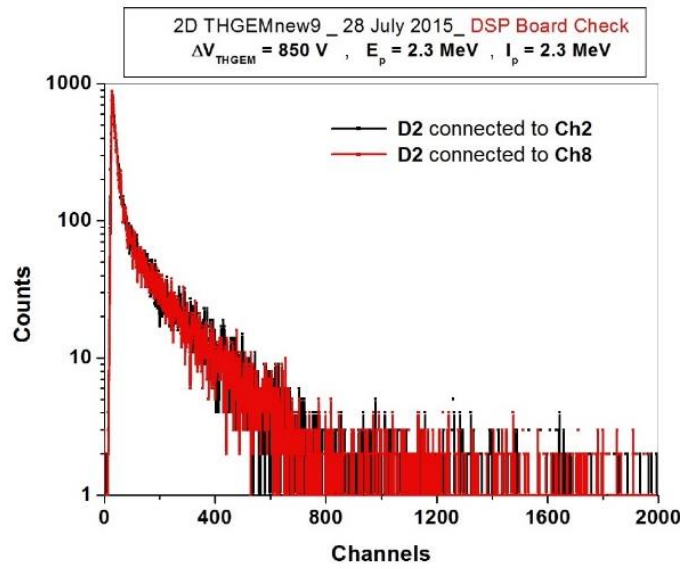

b. 


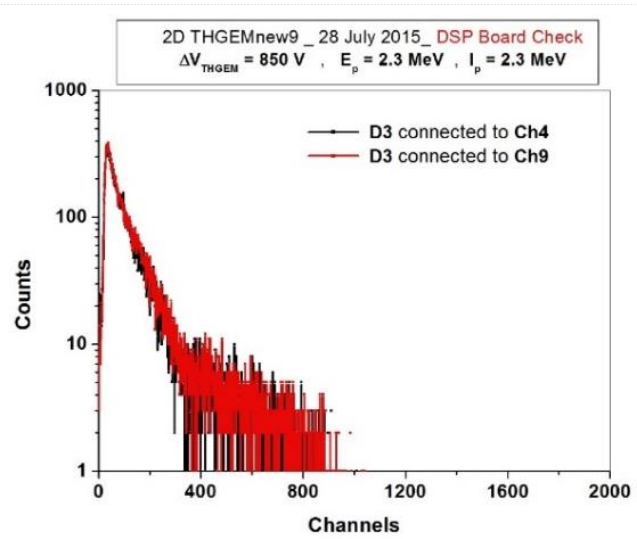

c.

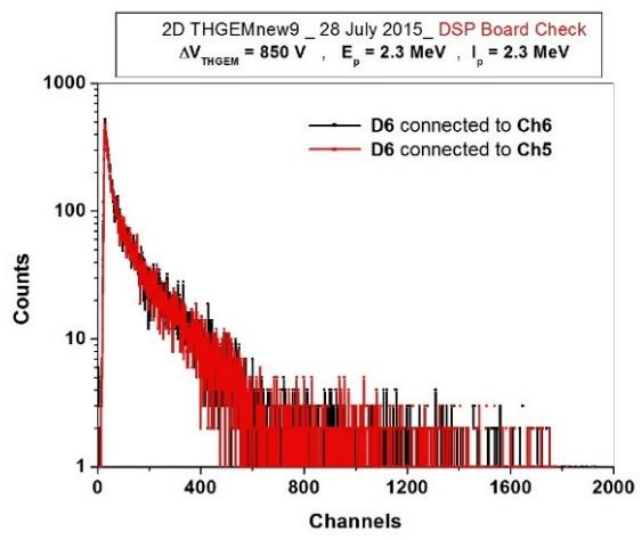

e.

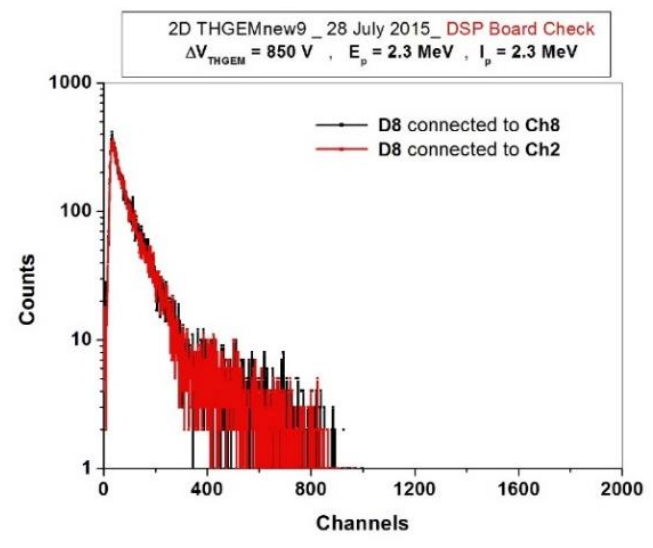

g.

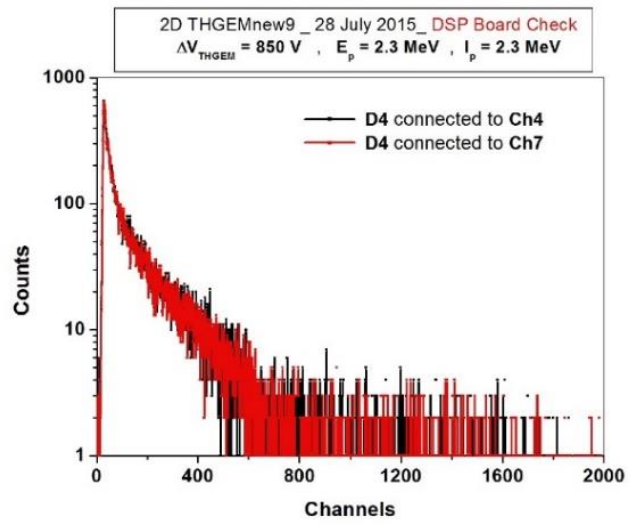

d.

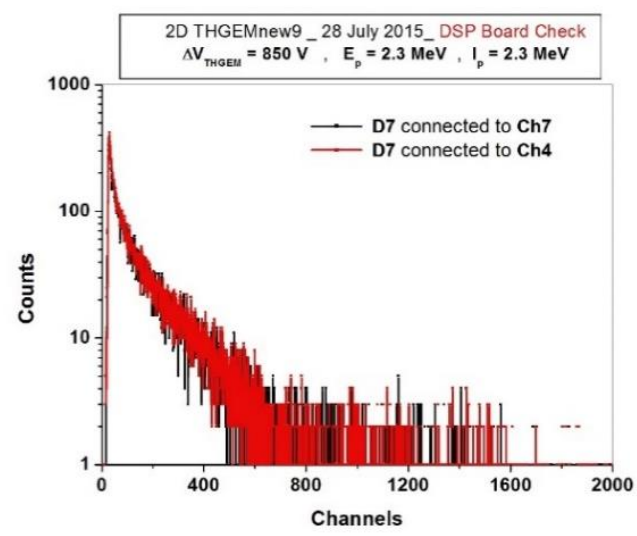

f.

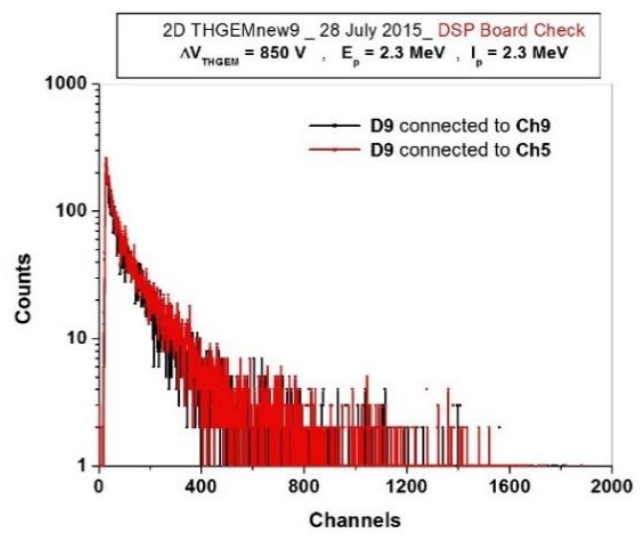

h.

Fig. 7.32 MMI-DSP channels connecting individually to different detectors to check each channel on each board, a. THGEM detector (D1) raw spectrum acquired using Ch1 and Ch5 on the MMI-DSP, b. D2 raw spectrum acquired using Ch2 and Ch8, c. D3 raw spectrum acquired using Ch4 and Ch9, d. D4 raw spectrum acquired using Ch4 and Ch7, e. D6 raw spectrum acquired using Ch6 and Ch5, f. D7 raw spectrum acquired using $\mathbf{C h} 7$ and Ch4, g. D8 raw spectrum acquired using $\mathrm{Ch} 8$ and $\mathrm{Ch} 2$, h. D9 raw spectrum acquired using $\mathrm{Ch} 9$ and $\mathrm{Ch} 5$ 


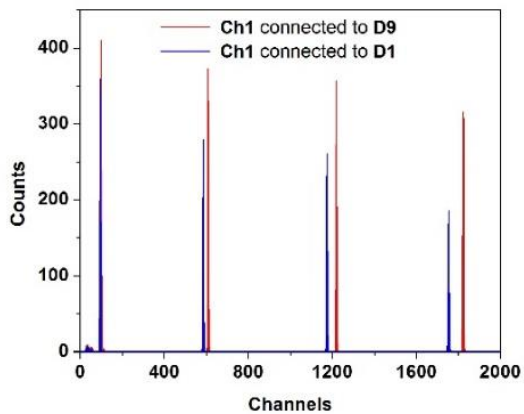

a.

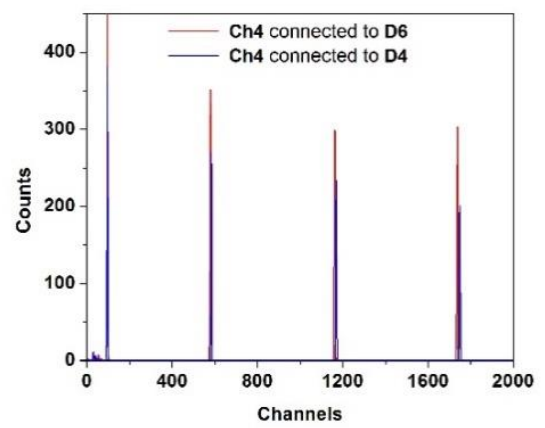

c.

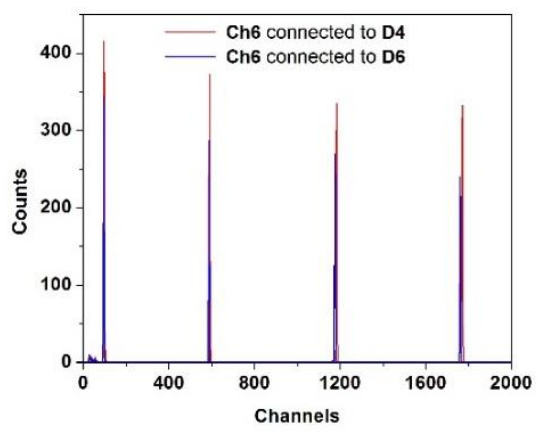

e.

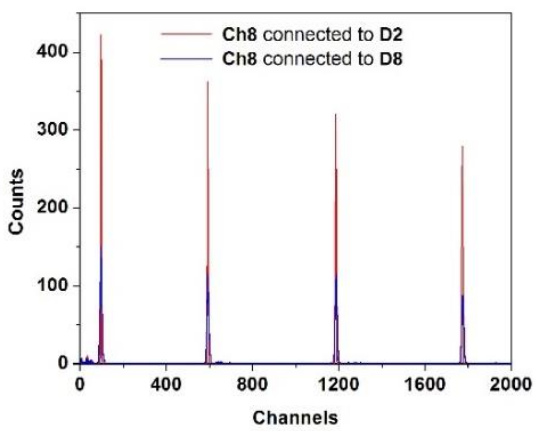

g.

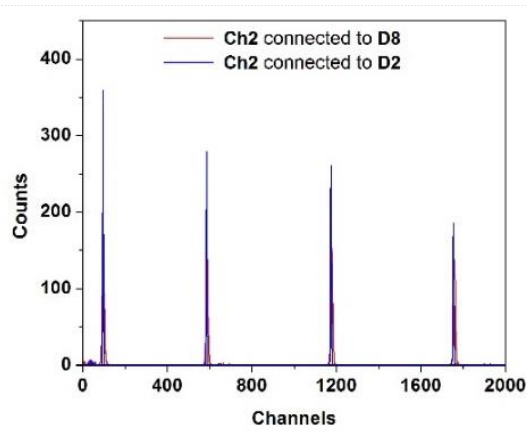

b.

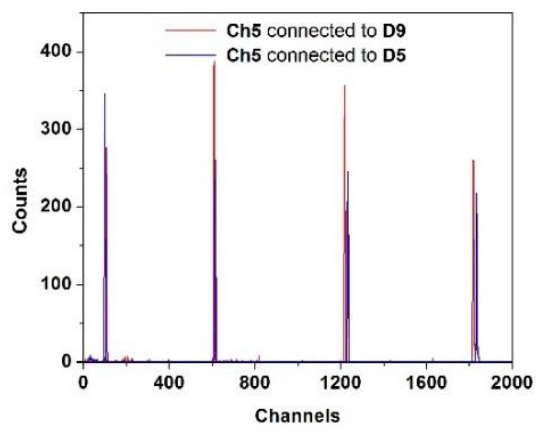

d.

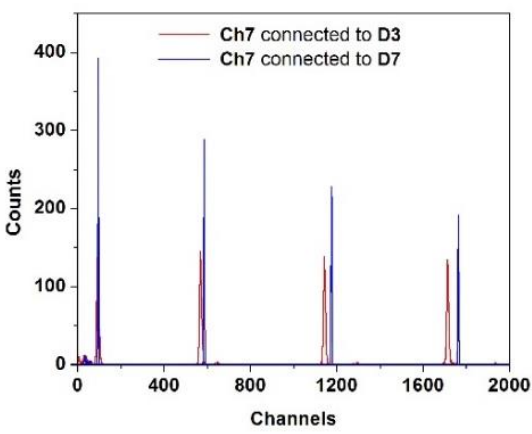

f.

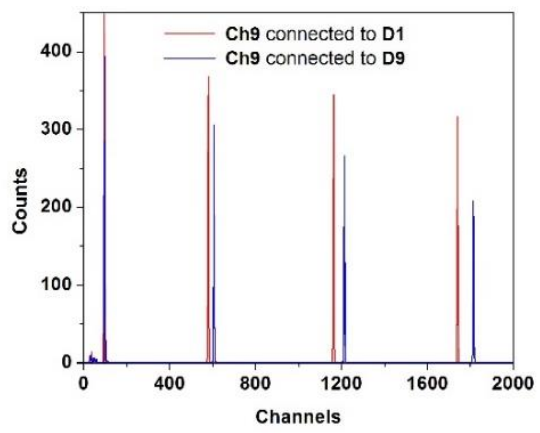

h.

Fig. 7.33 Pulser calibration for each MMI-DSP channels connecting to different detectors a. Ch1, b. Ch2, c. $\mathrm{Ch} 4$, d. $\mathrm{Ch} 5$, e. $\mathrm{Ch} 6$, f. $\mathrm{Ch} 7$, g. $\mathrm{Ch} 8$, h. $\mathrm{Ch} 9$ 


\section{D Detector Rotation Effect Assessment}

Care was taken consistently to place the detector inside the irradiation cavity in the same spot for any measurement carried out with the neutron beam using the Tandetron Accelerator irradiation facility. However, a set of experiments was performed using THGEM \#11 to explore the effect of rotating the detector at its position. Fig. 7.34 displays the detector position inside the cavity for both cases. As can be seen, after $90^{\circ}$ clockwise rotation (from the view of the neutron beam), D7 became at the position of D1, D4 at D2, D1 at D3 and so on. D5 position did not change. Figs. 7.35 and 7.36 show the raw and microdosimetric spectra for each detector for $\mathrm{E}_{\mathrm{p}}=2.3 \mathrm{MeV}$ and $\mathrm{I}_{\mathrm{p}}=50 \mu \mathrm{A}$ in both positions. It was observed that the spectra of the detectors at the regular position and their corresponding $90^{\circ}$ rotated position were in agreement. Note that the spectrum was not acquired for D7 in regular position, so the comparison to D9 in rotated case was excluded.

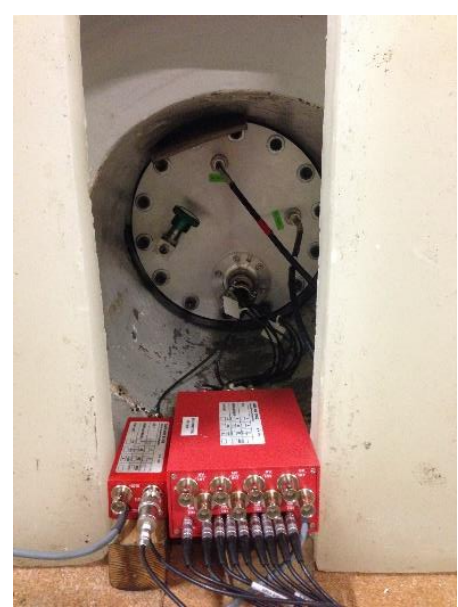

a.

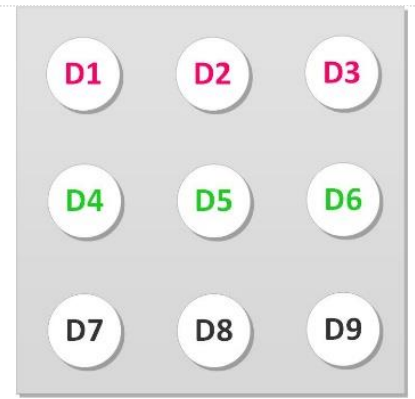

c.

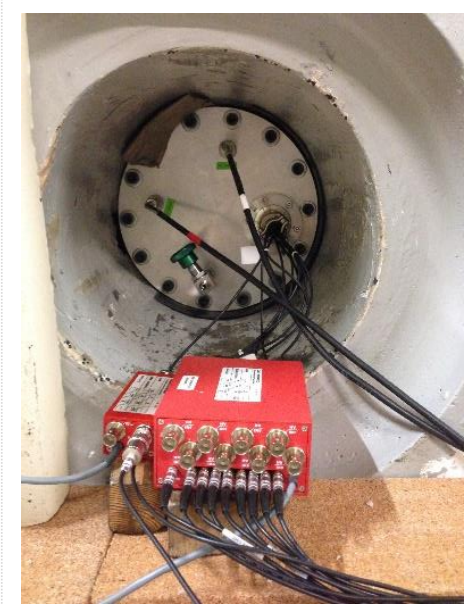

b.

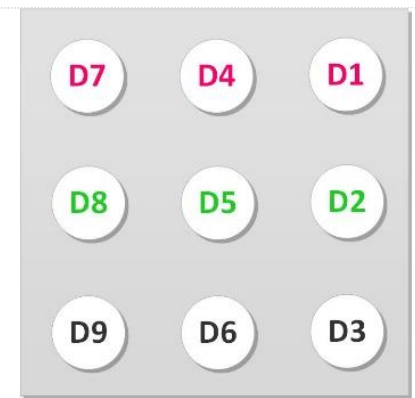

d.

Fig. 7.34 2D THGEM detector position inside the irradiation cavity at a \& c. regular position and $\mathbf{b} \& \mathbf{d}$. $90^{\circ}$ rotated position 


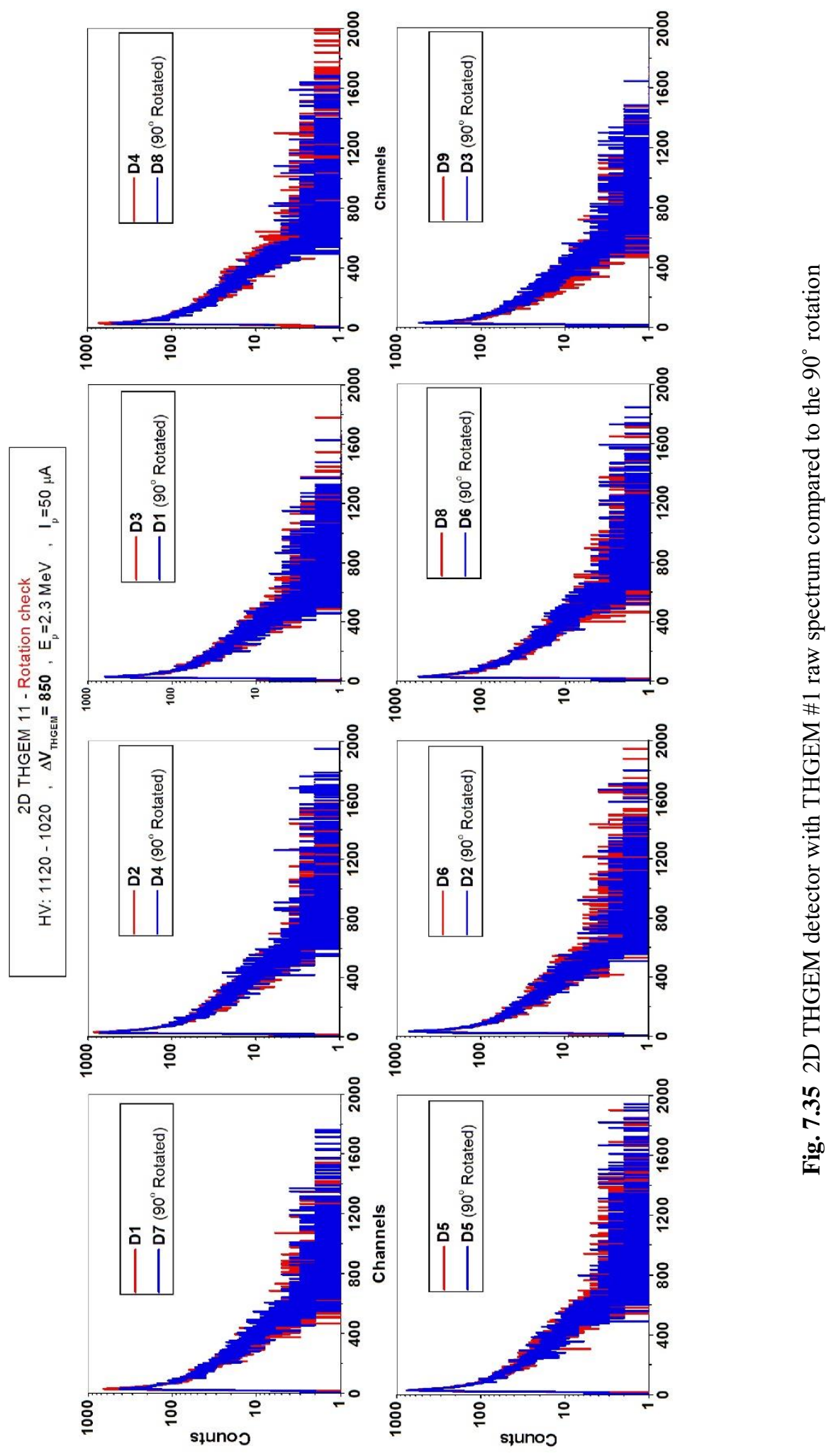




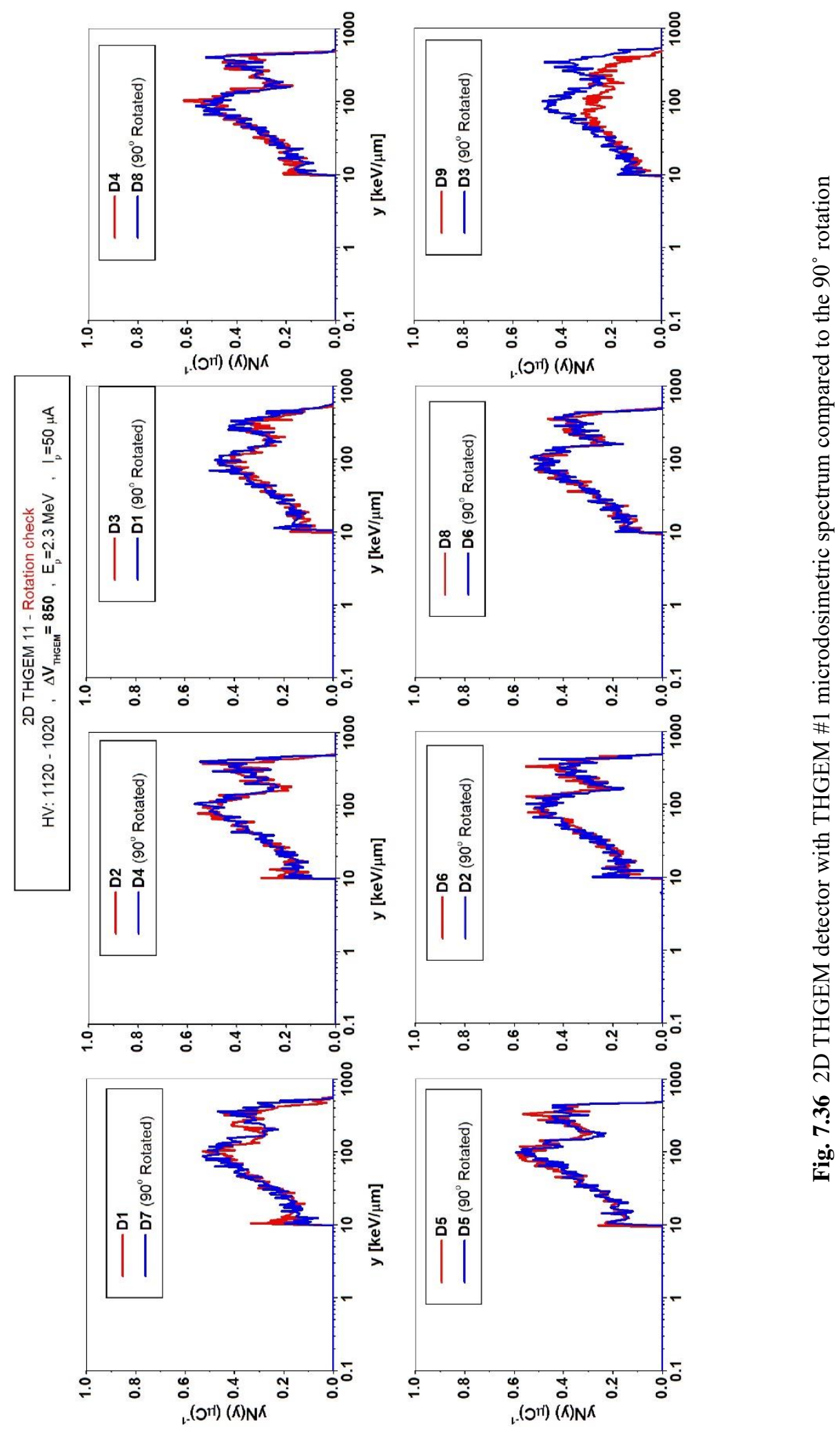




\section{D Detector Response of the 3 Different THGEMs for $E_{p}=2.3 \mathrm{MeV}$ and $I_{p}=50 \mu A$}

The 2D detector response was investigated for 3 different THGEMs (\#9, \#10 and \#11) for the same proton energy $\left(E_{p}=2.3 \mathrm{MeV}\right)$ and current $\left(I_{p}=50 \mu \mathrm{A}\right)$. The measurements were performed individually with the same experimental setup and high voltage settings. Every time the detector had to be opened to replace the THGEM, and then the chamber was closed, sealed, pumped down and refilled again with the TE gas to the same pressure. For each measurement, it was attempted to keep the settings as identical as possible.

The microdosimetric spectra for each of these measurements were compared in Figs. 7.37 and 7.38. The microdosimetric spectra for all the 3 THGEMs were consistent in all the detectors except for D3 and D8. It can be seen that the response of the D3 and D8 for THGEM \#9 is different. For this THGEM the proton peak height is about 1.5 times higher than the other two THGEMs. As this was not repeated for the other two measurements, it could be related to the THGEM \#9 failure in those particular regions corresponding to the D3 and D8, or it could be the functionality failure of ch3 and ch8 on that particular day. Considering THGEM \#9 as not a good working one, the results for the THGEM \#10 and \#11 were remarkably acceptable and consistent.

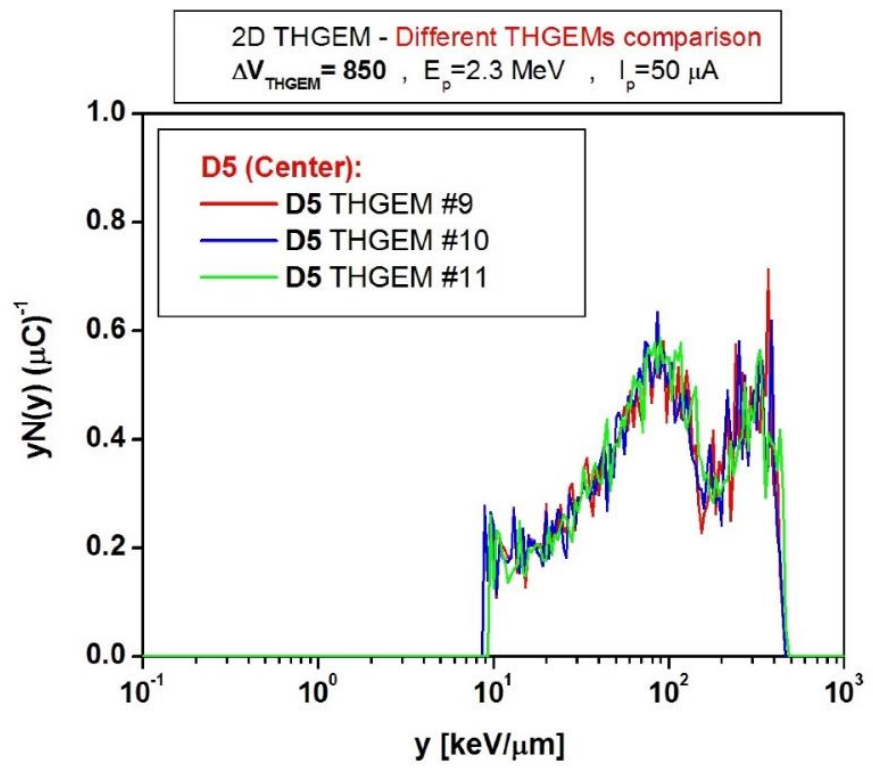

Fig. 7.37 Comparison of the THGEM detector (D5) microdosimetric response acquired with different THGEMs (\#9, \#10 and \#11) for $E_{p}=2.3 \mathrm{MeV}$ and $\mathrm{I}_{\mathrm{p}}=50 \mu \mathrm{A}$ 


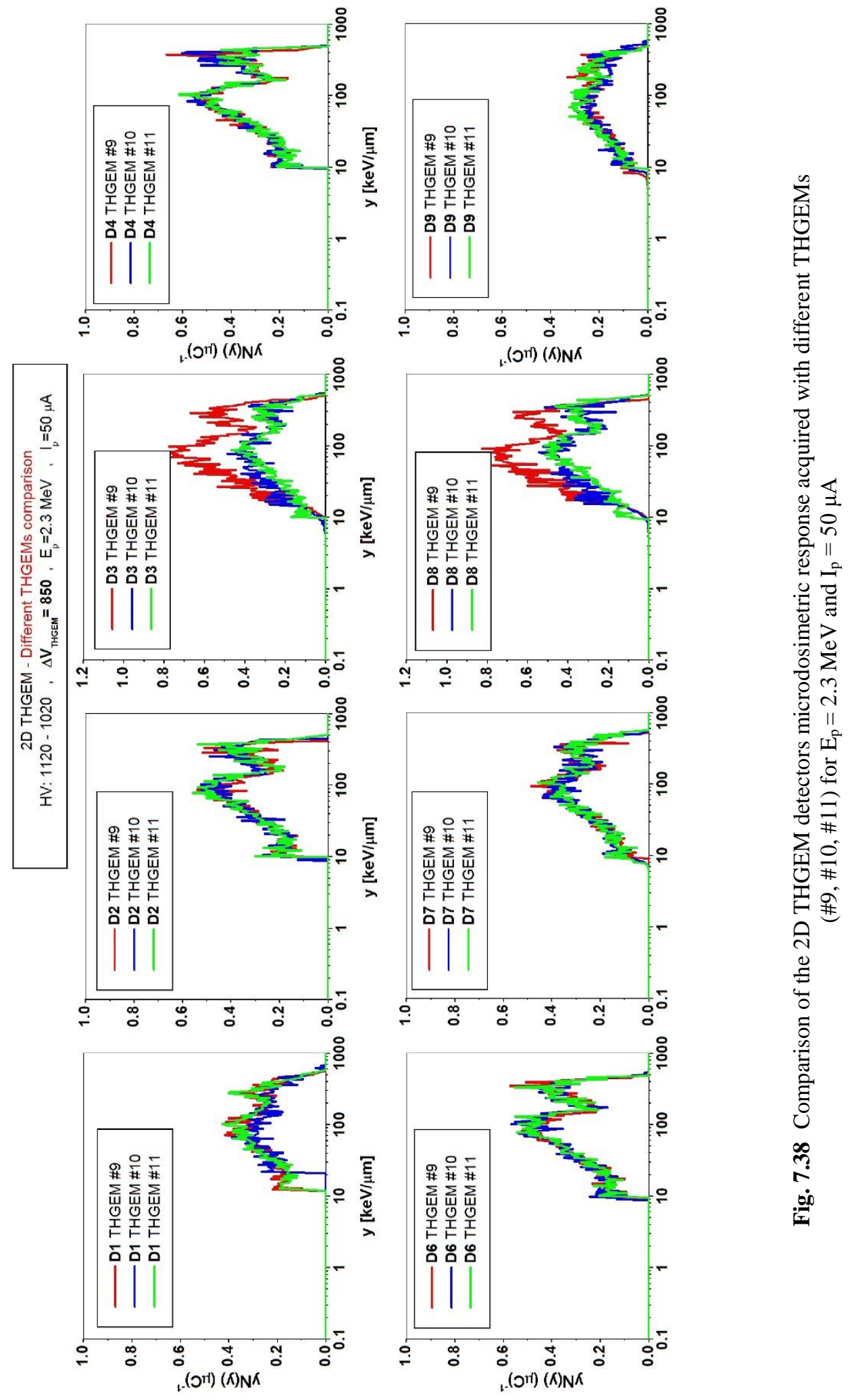




\section{D Detector Response of THGEM \#10 for $E_{p}=2.3 \mathrm{MeV}$ and Different Currents}

The detector response was studied for THGEM \#10 with the same proton energy of 2.3 $\mathrm{MeV}$ but for 2 different proton currents of $50 \mu \mathrm{A}$ and $150 \mu \mathrm{A}$. The raw spectra are shown in Fig. 7.39. To compare the effect of increasing the proton current on the events count rate, it was attempted first to evaluate the region of the spectra above the noise level. Fig. 7.40 shows the raw spectra in the low channel region (0 to 100). The cut of lineal energy was set to $10 \mathrm{keV} / \mu \mathrm{m}$ as before and the channels corresponding to the lineal energy lower than $10 \mathrm{keV} / \mu \mathrm{m}$ were determined using the proper pulser calibration and are reported in table 7-6. The events for channels below to the upper bound of this bin were considered as the noise and were subtracted for each detector. Then, the total count was calculated and divided by acquisition time to determine the count rate for all the detectors. In Fig. 7.41 the count rate of each detector was compared for $\mathrm{I}_{\mathrm{p}}=50 \mu \mathrm{A}$ and $\mathrm{I}_{\mathrm{p}}=150 \mu \mathrm{A}$. As expected the count rate was increased about 3 times by increasing the current from $50 \mu \mathrm{A}$ to $150 \mu \mathrm{A}$. However, for D8, when the current was raised to $150 \mu \mathrm{A}$, an unexpected increase in the count rate and raw spectrum occurred for this THGEM. However, this issue was never observed with any other tested THGEM. 


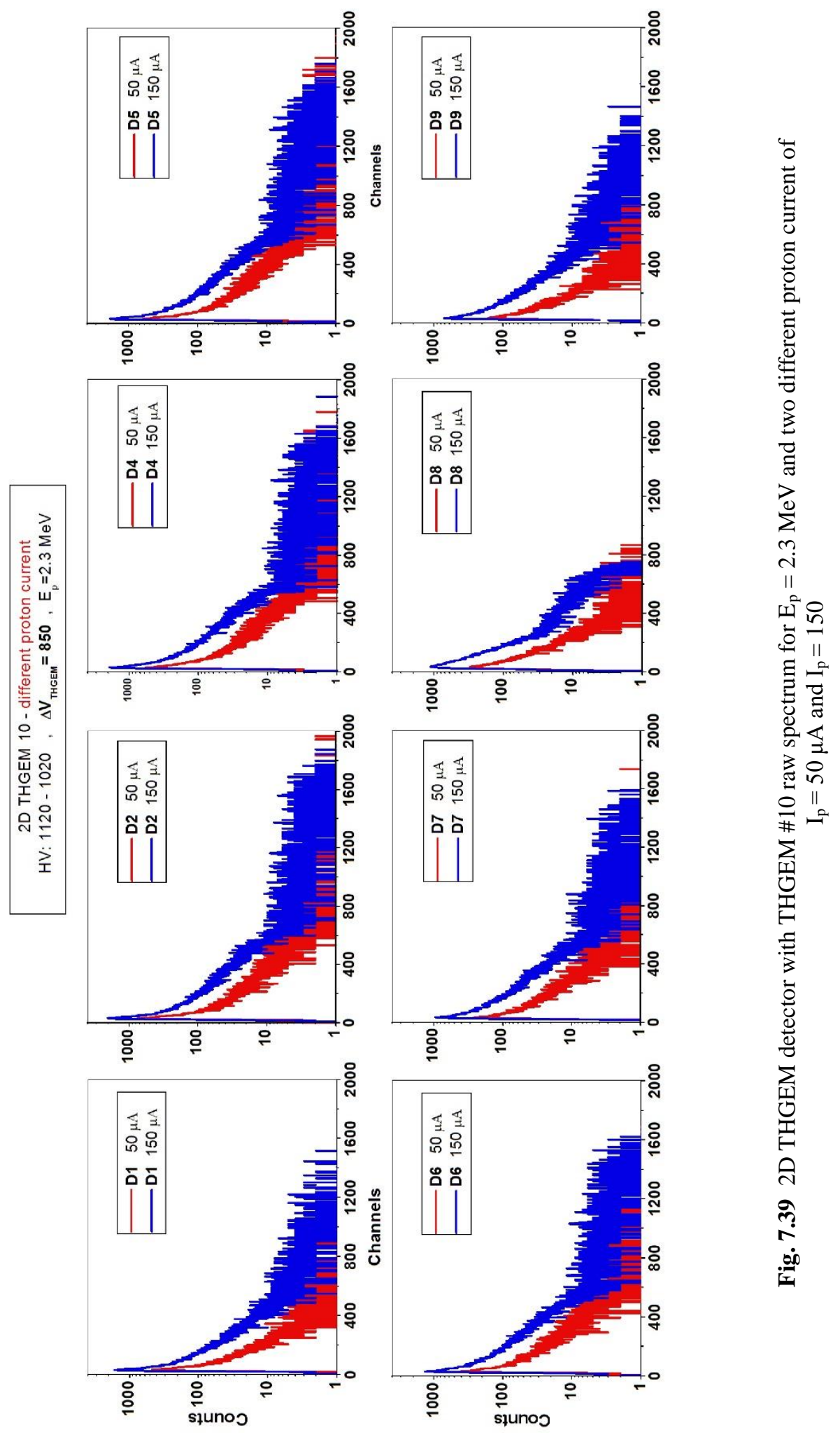




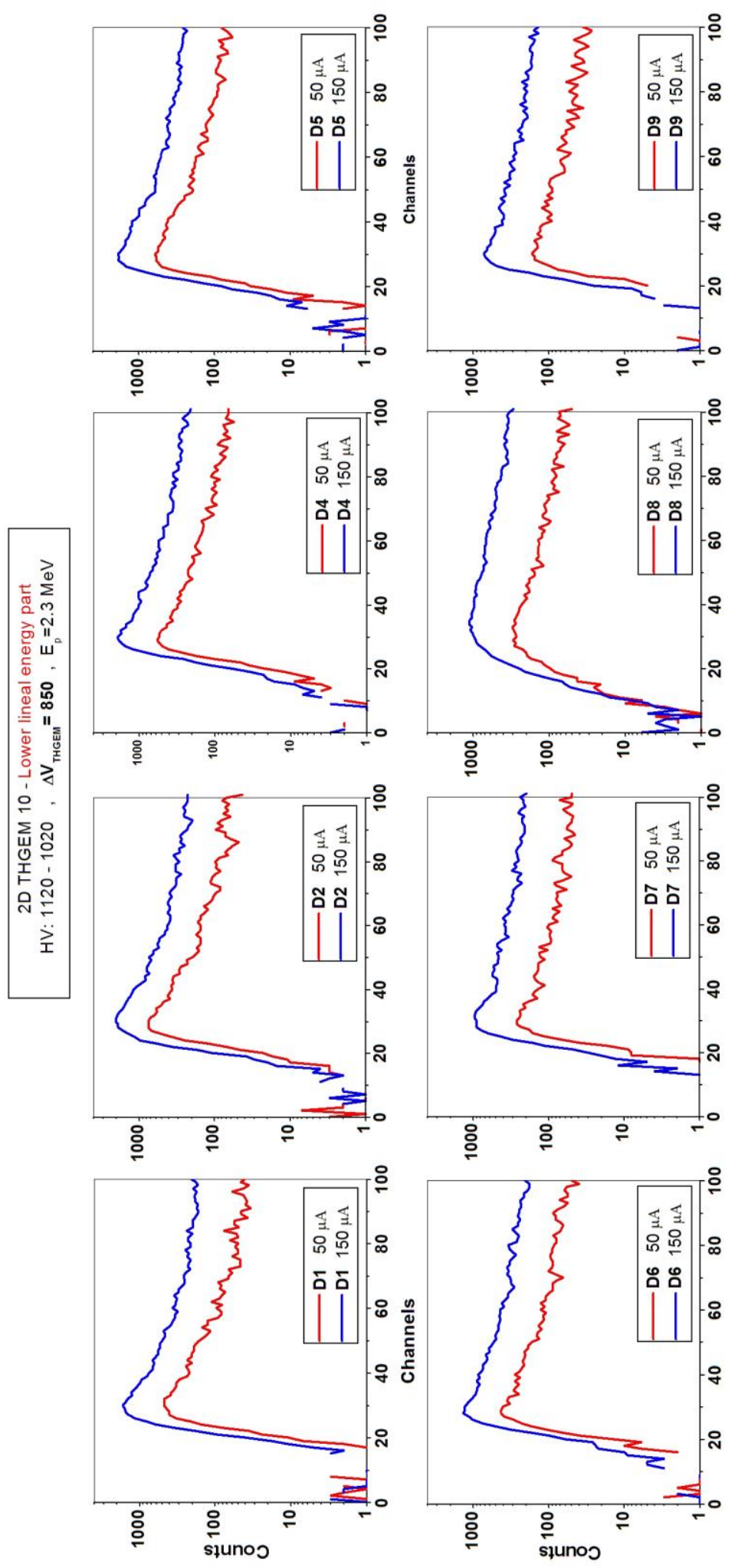

II 
Table 7-6 Channel numbers corresponding to the redistributed y value for each detector based on the related MMI-DSP input pulser calibration

\begin{tabular}{|c|c|c|c|c|c|c|c|c|c|c|c|}
\hline $\mathbf{y}$ & & & & D1 & D2 & D4 & D5 & D6 & D7 & D8 & D9 \\
\hline \multirow{2}{*}{10} & Ylow & 9.81 & Ch low & 29 & 36 & 38 & 40 & 33 & 32 & 12 & 29 \\
\hline & $\mathrm{y}_{\text {up }}$ & 10.96 & Ch up & 33 & 40 & 42 & 45 & 37 & 36 & 13 & 33 \\
\hline \multirow[b]{2}{*}{136} & $\mathrm{y}_{\text {low }}$ & 133 & Ch low & 429 & 524 & 515 & 543 & 476 & 476 & 190 & 429 \\
\hline & $\mathrm{y}_{\text {up }}$ & 149 & Ch up & 478 & 585 & 574 & 606 & 532 & 532 & 213 & 478 \\
\hline \multirow[b]{2}{*}{500} & Ylow & 491 & Ch low & 1582 & 1935 & 1893 & 1997 & 1758 & 1761 & 707 & 1582 \\
\hline & $\mathrm{y}_{\text {up }}$ & 547 & Ch up & 1765 & 2158 & 2111 & 2227 & 1961 & 1964 & 789 & 1765 \\
\hline
\end{tabular}

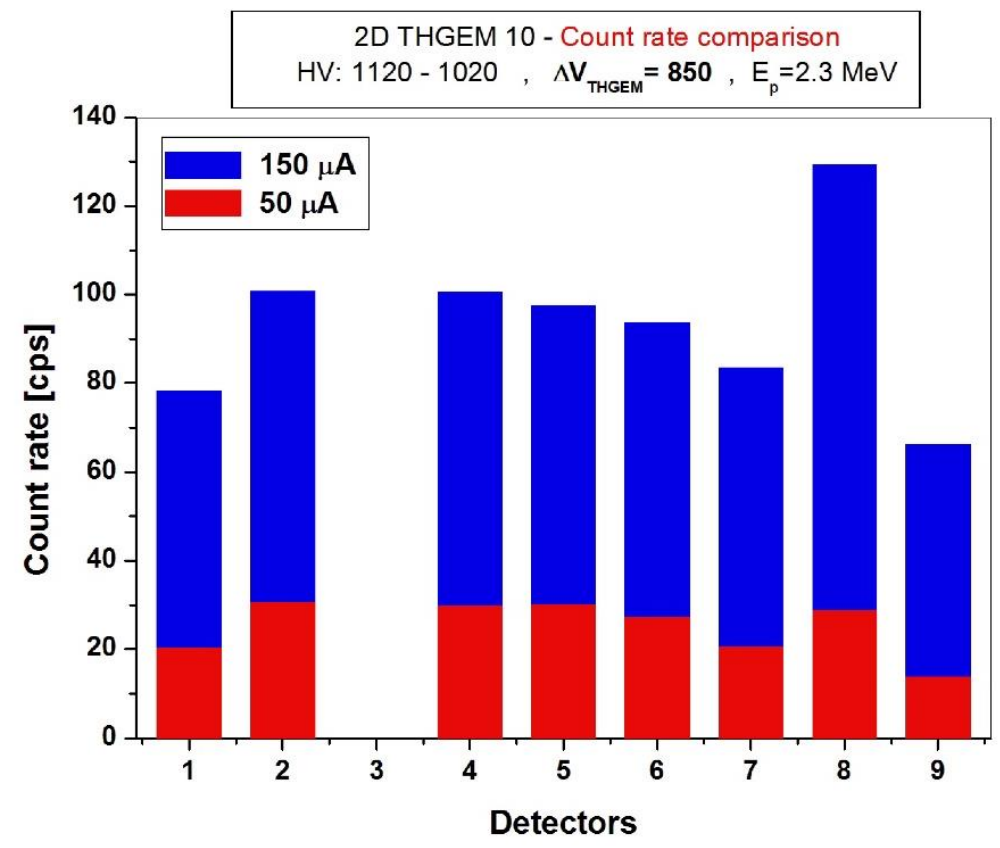

Fig. 7.41 Count rate comparison between $50 \mu \mathrm{A}$ and $150 \mu \mathrm{A}$ proton current $\left(\mathrm{E}_{\mathrm{p}}=2.3 \mathrm{MeV}\right)$ for all detectors (Except D3) 


\section{D Detector Response of THGEM \#10 for $I_{p}=50 \mu \mathrm{A}$ and Different Energies}

Next, the proton current was set to $50 \mu \mathrm{A}$ for different proton energies of 2, 2.3 and 2.5 $\mathrm{MeV}$. The detectors response are compared in Fig. 7.42. likewise, the noise part was subtracted for each detector and the total count rate was calculated and is shown in Fig. 7.43. All data are consistent except for the D8 count rate at $2.5 \mathrm{MeV}$ proton energy. There was an unexpected increase in the raw data and thus count rate for this detector at $2.5 \mathrm{MeV}$. However, the results for the other two energies were normal and followed the expected trend. This issue was never observed for this or any other detector, with any other tested THGEM. 


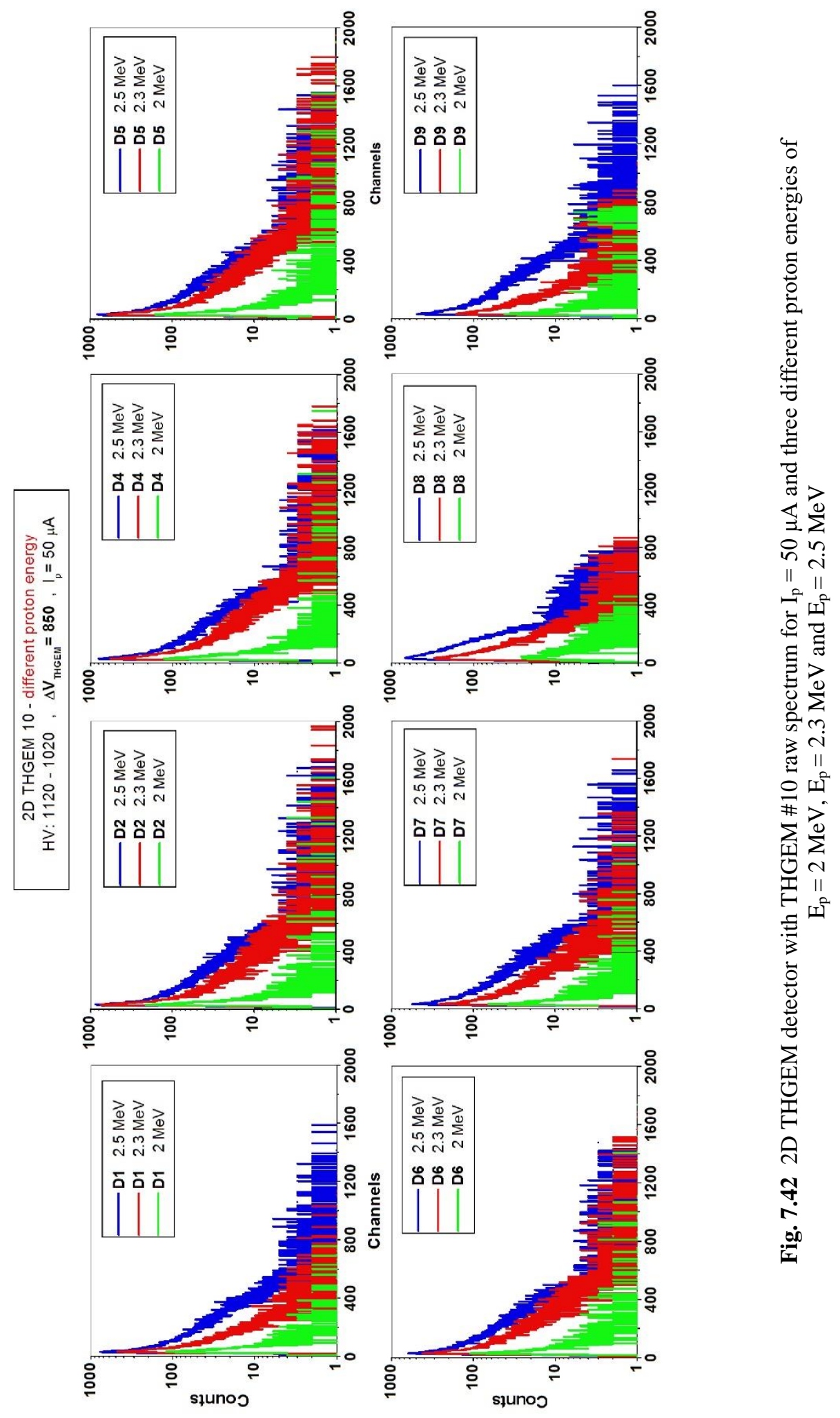




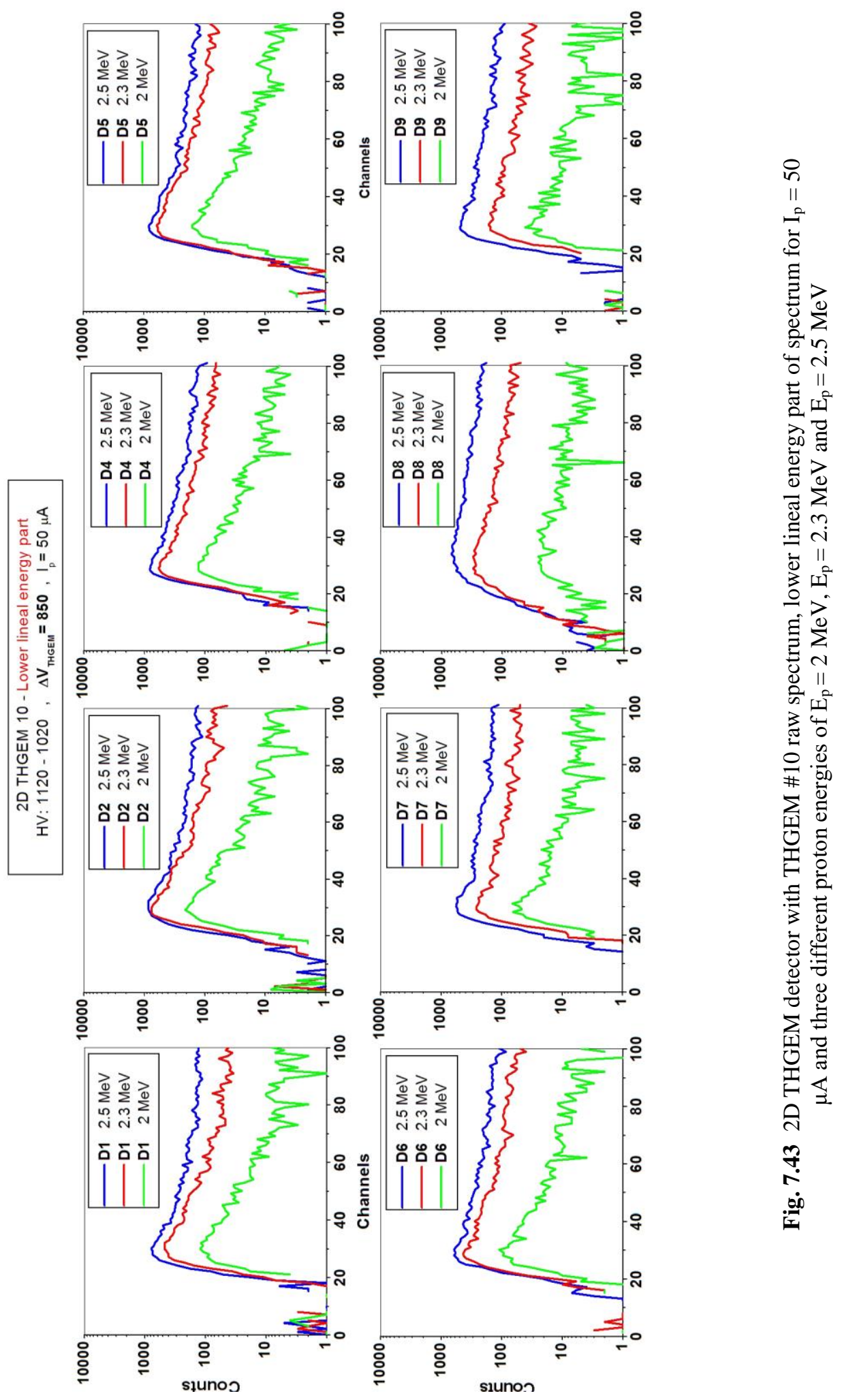




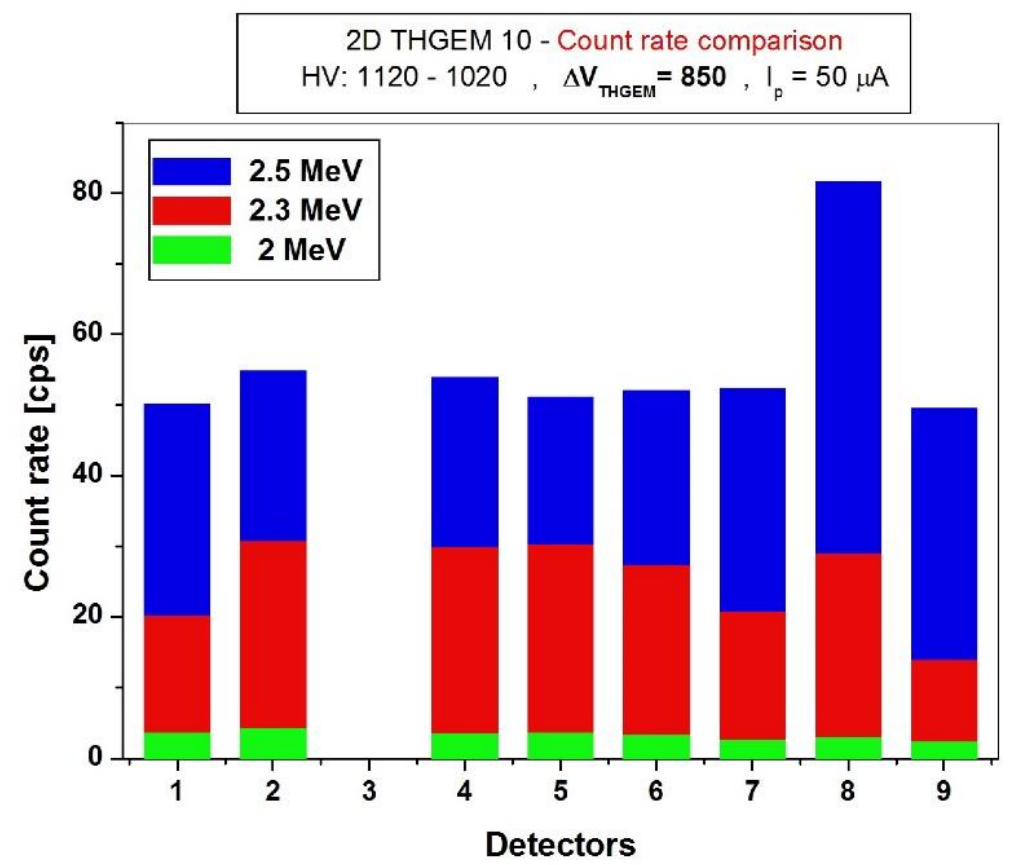

Fig. 7.44 Count rate comparison between $2 \mathrm{MeV}, 2.3 \mathrm{MeV}$ and $2.5 \mathrm{MeV}$ proton energies $\left(\mathrm{I}_{\mathrm{p}}=50 \mu \mathrm{A}\right)$ for all detectors (Except D3) 


\section{D Detector Response with 2 Different Induction Gaps}

In all the previous measurements the $1 \mathrm{~mm}$ Teflon spacers were used to create a gap between the readout board and THGEM layers. In order to check if the detector layers were all straight and no curvature happened due to the 4 single spacers, a new $1 \mathrm{~mm}$ thick square gap was fabricated from Rexolite plastic as shown in Fig. 7.45. In addition, to investigate the effect of the induction gap size on the response of the detector, a $2 \mathrm{~mm}$ thick square gap (double the $1 \mathrm{~mm}$ gap size) was fabricated.

Fig. 7.46 shows the microdosimetric spectrum of THGEM \#11 for both $1 \mathrm{~mm}$ spacer gap and $2 \mathrm{~mm}$ square gap. As can be seen, the spectra follow the same pattern and are consistent. No significant change was observed and thus the presence of the second peak could not be related to the size or type of the induction gap. In all the previous work by Orchard (2010), the $1 \mathrm{~mm}$ spacers were used as well, however, the square gap concept is the better option for the case of using double THGEM layers (which will be presented in the next subsection) to ensure that all the layers are straight.

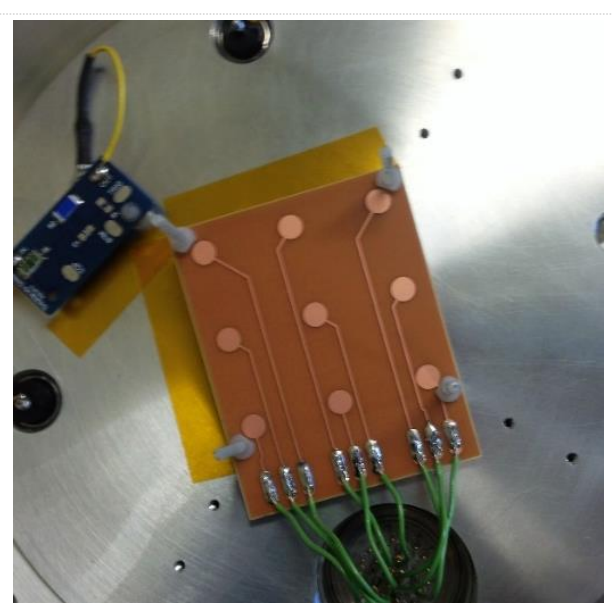

a.

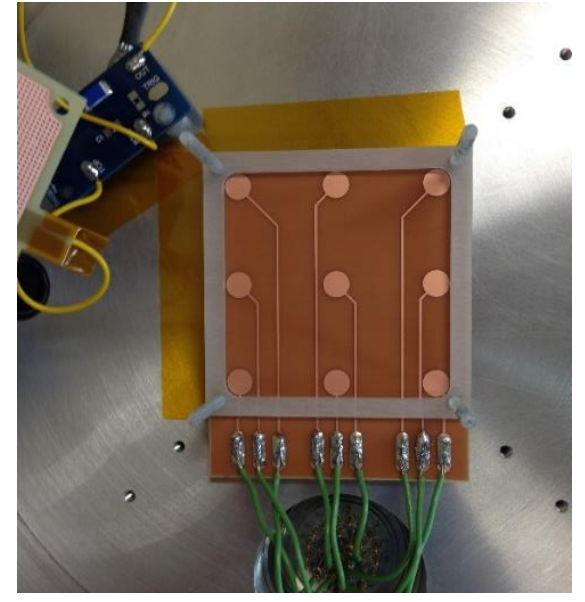

b.

Fig. 7.45 Two different induction gap (between readout board and THGEM layer) a. 1 mm spacer gap, b. $2 \mathrm{~mm}$ square gap 


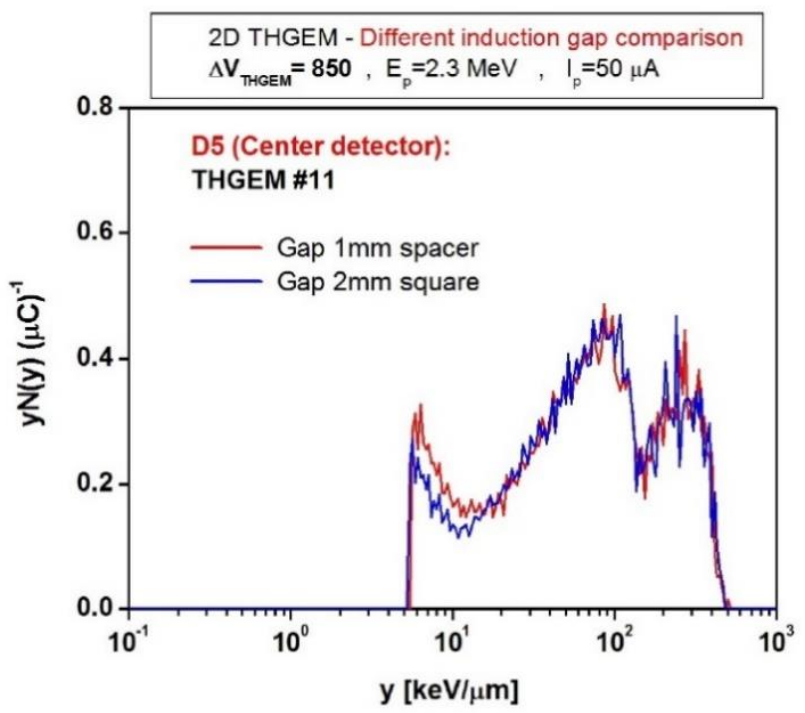

Fig. 7.46 Microdosimetric spectrum of THGEM \#11 acquired with ORTEC DSPEC (16k) for two different induction gap size

\subsubsection{D Double-Layer THGEM Detector Performance}

Having a small size detector sensitive volume requires having a high multiplication gain to achieve a signal with a reasonable signal to noise ratio. It has been shown that using a multiple-GEM arrangement (for different applications) has the advantage of increase in gain without enormous high voltage across the THEGM (Altunbus et al, 2002; Ketzer et al, 2004; Shalem et al, 2006; Mia et al, 2007; Cortesi et al, 2007; Breskin et al, 2010; Ohshita et al, 2010; Hanu et al, 2015). Hence, the double-THGEM arrangement was assembled as explained in chapter $2(2.7 .2 .1)$ and the detector response has been investigated. THGEM \#11, which was used in the last measurement of the single-THGEM arrangement, was assembled together with THGEM \#12 for a double-THGEM detector. The $2 \mathrm{~mm}$ square gap was used between the two THGEMs. Likewise, a $1 \mathrm{~mm}$ square gap fabricated from Rexolite was placed between the bottom THGEM and the readout board. Table 7-7 summarizes the number of measurements that have been carried out for single and double THGEM arrangements with the two types of the gap. The detector was placed inside the irradiation cavity in front of the neutron beam. The signal of the detector (D5) was monitored and compared to the single layer THGEM arrangement as shown in Fig. 
7.47. The applied high voltage was divided between the two THGEMs' top and bottom layers according to the circuit diagram 2.15.

Table 7-7. Measurements carried out for single layer THGEM \#11 and double layers THGEM \#11 \& \#12 for different induction gap size and type

\begin{tabular}{|c|c|c|c|c|c|}
\hline \multirow{2}{*}{\multicolumn{2}{|c|}{ THGEM 11}} & \multicolumn{2}{|c|}{ Collection Gap } & & \\
\hline & & Size & Type & & \\
\hline \multirow{3}{*}{ Single } & 7 Oct 2015 & $1 \mathrm{~mm}$ & spacers & & \\
\hline & 9 Oct 2015 & $1 \mathrm{~mm}$ & spacers & & \\
\hline & 4 Nov 2015 & $2 \mathrm{~mm}$ & square & & \\
\hline \multirow{2}{*}{ THGEM } & \multirow{2}{*}{$11 \& 12$} & \multicolumn{2}{|c|}{ collection Gap } & \multicolumn{2}{|c|}{ Gap between THGEMs } \\
\hline & & Size & Type & Size & Type \\
\hline \multirow{4}{*}{ Double } & 3 Dec 2015 & $1 \mathrm{~mm}$ & square & $2 \mathrm{~mm}$ & square \\
\hline & 10 Dec 2015 & $1 \mathrm{~mm}$ & square & $2 \mathrm{~mm}$ & square \\
\hline & 30 Mar 2016 & $1 \mathrm{~mm}$ & square & $2 \mathrm{~mm}$ & square \\
\hline & 1 April 2016 & $1 \mathrm{~mm}$ & square & $2 \mathrm{~mm}$ & square \\
\hline
\end{tabular}

The maximum high voltage that could be applied across the THGEM layer was $733 \mathrm{~V}$ for double and $850 \mathrm{~V}$ for a single layer. However, the detector signal amplitude was 10 times higher by applying about a $120 \mathrm{~V}$ less high voltage across each THGEM for the double layers. The effective gain of the single and double THGEMs was previously compared in the TE-propane gas at 167 torr, measured with a ${ }^{244} \mathrm{Cm}$ source by Hanu (2013). Three times higher maximum effective gain was reported for the double-THGEM configuration compared to the single THGEM.

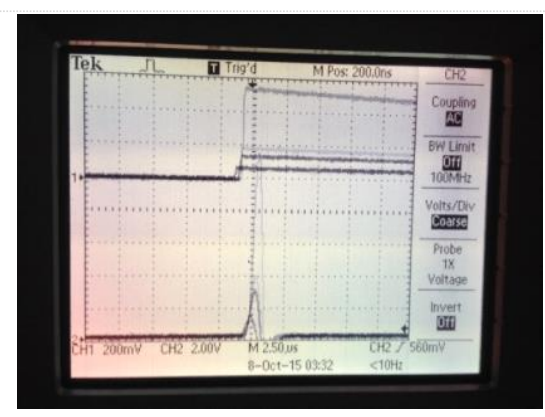

a.

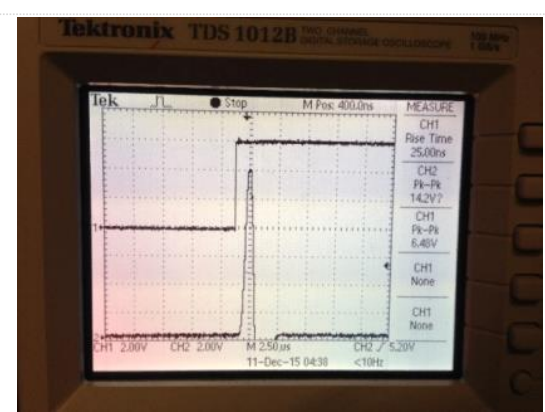

b.

Fig. 7.47 The THGEM detector (D5) signal response for a. single-layer THGEM \#11 with $\Delta \mathrm{V}_{\mathrm{THGEM}}=850$ $\mathrm{V}\left(\mathrm{HV}_{\text {Cathode }}=1120 \mathrm{~V}\right.$ and $\left.\mathrm{HV}_{\text {THGEM }}=1020 \mathrm{~V}\right)$ : preamplifier $(\mathrm{CAEN})($ channel 1) and amplifier $($ gain $=20)$ (channel 2), b. double-layer THGEM \#11 \& \#12 with $\Delta \mathrm{V}_{\text {THGEM1 } \& 2}=733 \mathrm{~V}\left(\mathrm{HV}_{\text {Cathode }}=1760 \mathrm{~V}\right.$ and $\left.\mathrm{HV}_{\text {THGEM }}=1660 \mathrm{~V}\right)$ : preamplifier $(\mathrm{CAEN})$ and amplifier $($ gain $=2)$ 
The microdosimetric spectrum of the central detector (D5) for the single and double layer THGEMs is compared in Fig. 7.48. The 1/2" TEPC spectrum, which was acquired at the position of the 2D THGEM detector inside the irradiation cavity in Oct 2014, was plotted as a reference. The best result with the THGEM detector, which was acquired with THGEM \#5 in April 2015, was also plotted for the comparison of the lower lineal energy region. As can be seen, the shape of the spectrum for the single THGEM \#11 and double THGEMs \#11 and \#12 are consistent above $15 \mathrm{keV} / \mu \mathrm{m}$. The higher lineal energy cut off for the double THGEMs arrangement was due to the fact that the gain was too high to register all the events, even though the DSPEC gain was set to the lowest possible value. This was not a concern at this stage as it was believed that any events registered after the proton edge were not physically possible and were due to a systematic issue, which has to be addressed eventually. The lower lineal energy region of the spectra, which is related to the gamma-ray emission, followed the same pattern but not quantitatively.

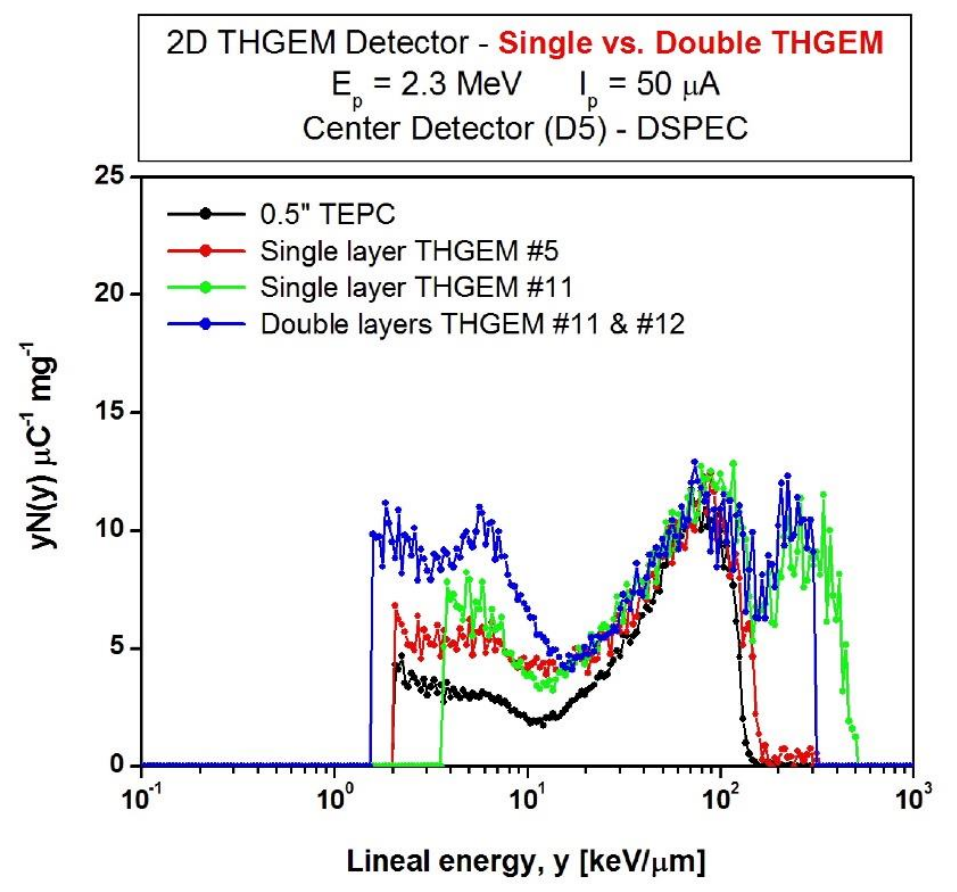

Fig. 7.48 Microdosimetric spectra acquired with ORTEC DSPEC (16k) for single-layer THGEM \#11 vs double-layer THGEM \#11 and \#12 
The discrepancy between the 1/2" TEPC and THGEM detector gamma-ray part could be due to the fact that the data were acquired in different times (about a year after). Also, most of the THGEM detector layers consist of Copper $(\mathrm{Cu})$, which get activated during the irradiation time. The neutron cross section for stable ${ }^{63} \mathrm{Cu}$ is about 4.5 barns and $\mathrm{Cu}$ gets activated through the ${ }^{63} \mathrm{Cu}(\mathrm{n}, \gamma){ }^{64} \mathrm{Cu}$ reaction with a half-life of $12.7 \mathrm{~h}$. This could be the explanation for the increase in the level of gamma-rays in the 2D THGEM detector compared to 1/2" TEPC. Also, the double THGEM arrangement has more $\mathrm{Cu}$ inside the detector and this could justify the higher level of gamma-rays in double THGEM compared to single THGEM configuration.

To investigate this matter, a set of measurements was carried out with double THGEMs and the background radiation was monitored at the beginning, during and at the end of the $4 \mathrm{~h}$ long measurement. Generally, the detector was irradiated with $E_{p}=2.3 \mathrm{MeV}$ and $I_{p}=$ $50 \mu \mathrm{A}$ initially, to observe the detector signal growth on the oscilloscope by increasing the applied high voltage. This procedure may take up to $20 \mathrm{~min}$ until the detector reaches its operating voltage. Therefore, the $\mathrm{Cu}$ could get activated sooner. However, in order to monitor the background level, the background was first collected before the beam was switched on, and then the measurement started with the lower proton energies. Initially, proton energy was set to $1.8 \mathrm{MeV}$, which is below the threshold for neutron production. The detector high voltage was set to the operating high voltage found in previous measurements for double THGEMs $\left(\mathrm{HV}_{\text {Cathode }}=1800 \mathrm{~V}\right.$ and $\left.\mathrm{HV}_{\text {Cathode }}=1700 \mathrm{~V}\right)$. The spectrum was collected for $600 \mathrm{sec}$. The gross count rate was $10 \mathrm{cps}$. Then, the proton energy was increased to $2 \mathrm{MeV}$ and the spectrum was collected for $600 \mathrm{sec}$. Immediately after this measurement, when the beam was off, the background was collected and turned to be 4 cps (BKG 1). Afterward, the background was collected following each measurement with a raised proton energy. BKG 2 was collected after $2.1 \mathrm{MeV}$ data collection and BKG 3 was acquired after $2.3 \mathrm{MeV}$ data acquisition. The raw spectra of background data are shown in Fig. 7.49. The spectrum named BKG and BKGto are related to the case of background check before and after the high voltages applied to the detector and both before any beam was on. 


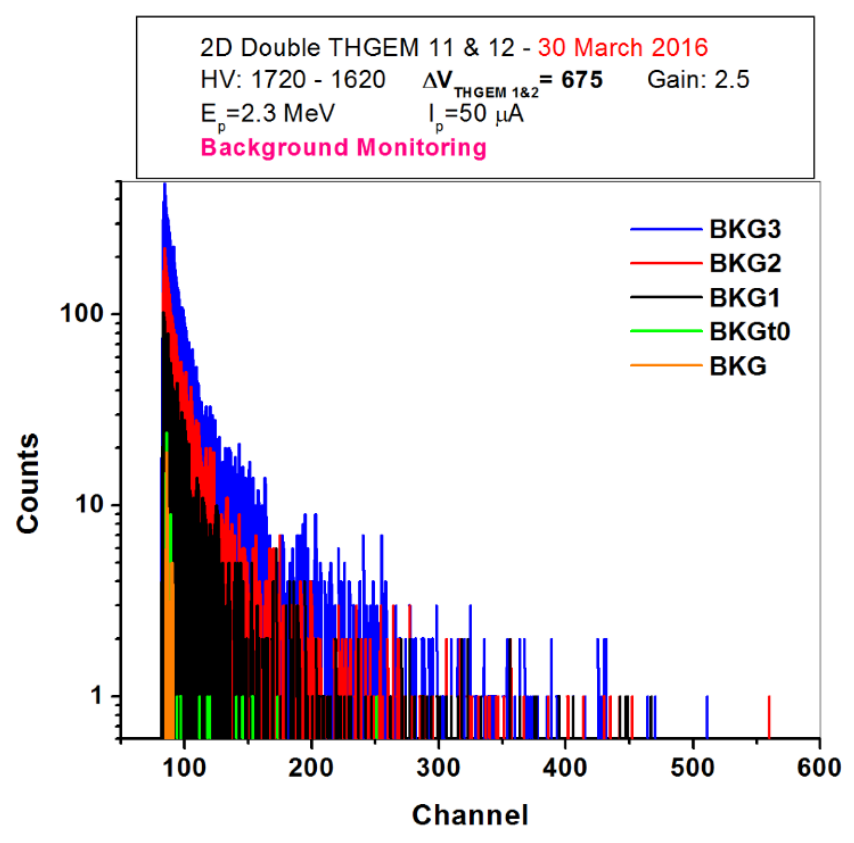

Fig. 7.49 Background Monitoring. Raw spectra acquired with double-layer THGEM \#11 and \#12

The analysis of the background spectra revealed that the background contribution was increased as the measurement proceeded (see table 7-8) and thus confirmed the higher amount of gamma-ray in the microdosimetric spectra of 2D THGEM detector compared to the $1 / 2 "$ TEPC.

Table 7-8. Count rate for background monitoring

\begin{tabular}{cccccc}
\hline & BKG & BKGt $_{0}$ & BKG1 & BKG2 & BKG3 \\
\hline $\begin{array}{c}\text { Count Rate } \\
{[\mathrm{cps}]}\end{array}$ & 0.14 & 0.23 & 4.43 & 9 & 20 \\
\hline
\end{tabular}

Going back to the double-THGEM detector as was discussed above for D5, the raw and microdosimetric spectra for all the other 8 detectors are presented in Fig. 7.50 and 7.51. The microdosimetric spectrum of each detector was plotted in comparison with the central detector (D5) as a reference, which was acquired by DSPEC and 16k ADC channels. The built in LLD level of MMI-DSP was set much higher, so the lower lineal energy region could not be registered. For future work this has to be changed. 


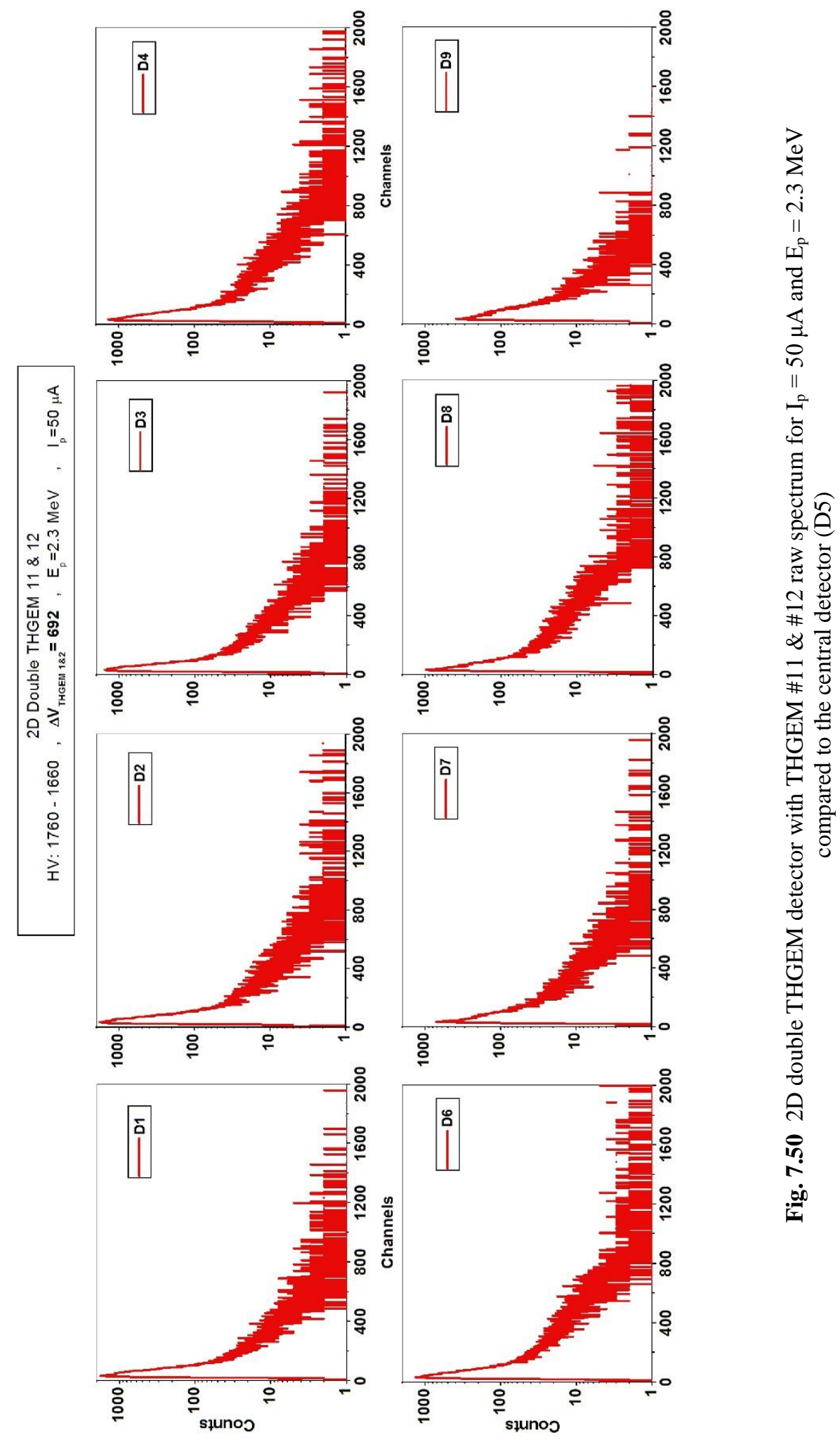




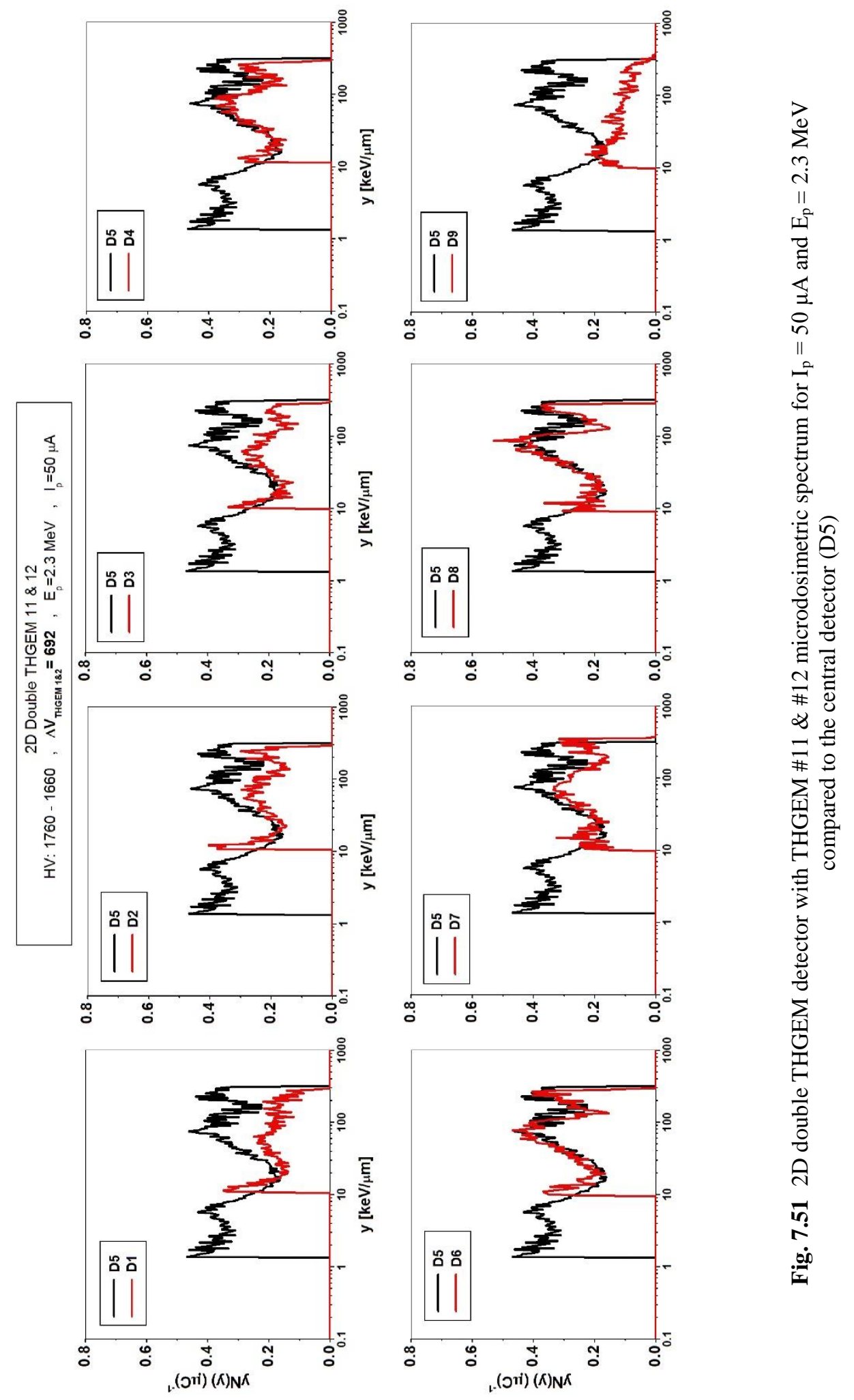




\subsection{Absorbed Dose Spatial Distribution}

The neutron absorbed dose can be calculated according to the methodology that was explained in chapter 5. First, the absorbed dose at the same position of 2D THGEM detector inside the irradiation cavity, measured by the standard 1/2" TEPC was calculated using e.q. 5-2 and table 5-1 data. The absorbed dose rates calculated for the 1/2" TEPC and the central THGEM detector (D5) for $\mathrm{E}_{\mathrm{p}}=2.3 \mathrm{MeV}$ are presented in table 7-9. The absorbed dose rates reported for the THGEM detector were obtained from different THGEMs and also single vs. double layer THGEMs to investigate the consistency of the results. The dose rate

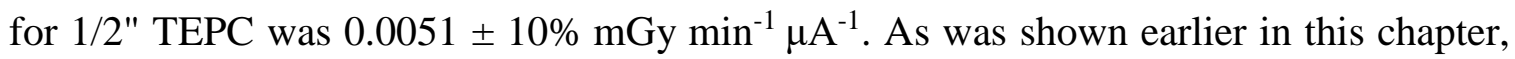
THGEM \#5 from the new batch had the best result significantly consistent with the 1/2" TEPC data. Therefore the dose rate was calculated for this THGEM and was $0.0062 \pm$ $0.0006 \mathrm{mGy} \mathrm{min}^{-1} \mu \mathrm{A}^{-1}$. The dose rate was calculated with THGEM \#11, the double THGEMs \#11 and \#12 from the new batch and THGEM \#1 from the old batch, whose spectra had the second extra peak. The absorbed dose rate was calculated both with and without the second peak to compare with the 1/2" TEPC and THGEM \#5 data.

Table 7-9. Dose rate for D5 obtained with different single and double layer THGEMs for $\mathrm{E}_{\mathrm{p}}=2.3 \mathrm{MeV}$.

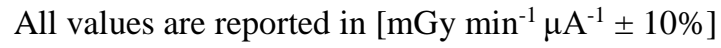

\begin{tabular}{|c|c|c|c|c|c|}
\hline & \multirow{3}{*}{$\begin{array}{c}\text { TEPC } \\
\mathbf{1 / 2} \text { " TEPC }\end{array}$} & \multicolumn{3}{|c|}{ New batch } & Old batch \\
\hline & & \multicolumn{2}{|c|}{ Single THGEM } & \multirow{2}{*}{$\begin{array}{c}\text { Double THGEM } \\
\text { \#11 \& \#12 }\end{array}$} & \multirow{2}{*}{$\begin{array}{c}\text { Single THGEM } \\
\# \mathbf{1}\end{array}$} \\
\hline & & $\# 5$ & \#11 & & \\
\hline $\begin{array}{c}\text { Dose Rate } \\
\text { w/o second peak }\end{array}$ & 0.0051 & 0.0062 & 0.0066 & 0.0064 & 0.0053 \\
\hline $\begin{array}{c}\text { Dose Rate } \\
\text { w second peak }\end{array}$ & - & - & 0.0098 & 0.0088 & 0.0078 \\
\hline
\end{tabular}

As can be seen that the old batch is not significantly different (without considering the second peak) to the TEPC measured dose, whereas the new batch gives dose results $20 \%$ higher than the TEPC. The mean value of the dose rate for all these 4 measurements is $0.00613 \mathrm{mGy} \mathrm{min}^{-1} \mu \mathrm{A}^{-1}$ with a standard deviation of $0.00050 \mathrm{mGy} \mathrm{min}^{-1} \mu \mathrm{A}^{-1}$. Between the two detectors, a percentage difference of $17 \%$ was observed in the measured absorbed 
dose rate. This difference was reported as $26 \%$ by Orchard (2010). The direct comparison of the values is not possible as the position of the detector inside the irradiation cavity in the two studies are different. As it was shown in chapter 6, the neutron dose rate is not uniform across the irradiation cavity and significantly varies by changing the detector position. The 2D THGEM detector in this study has 10-inch diameter (compare to the one in Orchard's study 5 inches), so there was not that much flexibility in terms of positioning the detector.

The THGEM detectors in this study overestimated the dose rate by $17 \%$ however the results reported by Orchard (2010) underestimated the dose rate by $26 \%$. The overestimation of the dose rate by the THGEM detectors in this study makes sense due to the broadening of the proton peak causing by the non-uniform THGEM holes multiplication. This issue can be easily solved if the THGEM fabrication technology improves.

The absorbed dose rate spatial distribution for the 9 positions (array of $3 \times 3$ gas cavities) inside the area of $6 \times 6 \mathrm{~cm}^{2}$ was determined by the dose calculation of each of the side and corner THGEM detectors. The center of each detector was at a $22.5 \mathrm{~mm}$ distance from the center of its adjacent detectors. The percentage difference between each detector and the central detector (D5) was reported in table 7-10 (from the dose rates without the second peak). The dose distribution was plotted in Fig. 7.52. D1 and D9 measured dose rates were significantly different from the center detector. As can be seen in Figs. 7.50 and 7.51 , the count rate for these two detectors is exceptionally low. It is believed that this is probably resulting from a systematic issue inside the detector during the assembly of the detector. As for this set of measurements, the square gap was used between the bottom THGEM and the readout board, so there is a possibility that part of the gap interfered with the collection pads corresponding to D1 and D9. 
Table 7-10. Dose rate distribution for D5 obtained with different single and double layer THGEMs for $\mathrm{E}_{\mathrm{p}}=2.3 \mathrm{MeV}$. All values are reported in $\left[\mathrm{mGy} \min ^{-1} \mu \mathrm{A}^{-1} \pm 10 \%\right]$

\begin{tabular}{cccccccccc}
\hline & D1 & D2 & D3 & D4 & D5 & D6 & D7 & D8 & D9 \\
\hline $\begin{array}{c}\text { Dose Rate } \\
\text { w/o second peak }\end{array}$ & 0.0033 & 0.0043 & 0.0045 & 0.0058 & 0.0064 & 0.0062 & 0.0056 & 0.0062 & 0.0027 \\
\hline $\begin{array}{c}\text { Dose Rate } \\
\text { W second peak }\end{array}$ & 0.0036 & 0.0060 & 0.0053 & 0.0067 & 0.0088 & 0.0074 & 0.0063 & 0.0075 & 0.0033 \\
\hline $\begin{array}{c}\text { \% difference } \\
\text { with D5 }\end{array}$ & 48 & 33 & 29 & 9 & - & 3 & 12 & 3 & 58 \\
\hline
\end{tabular}

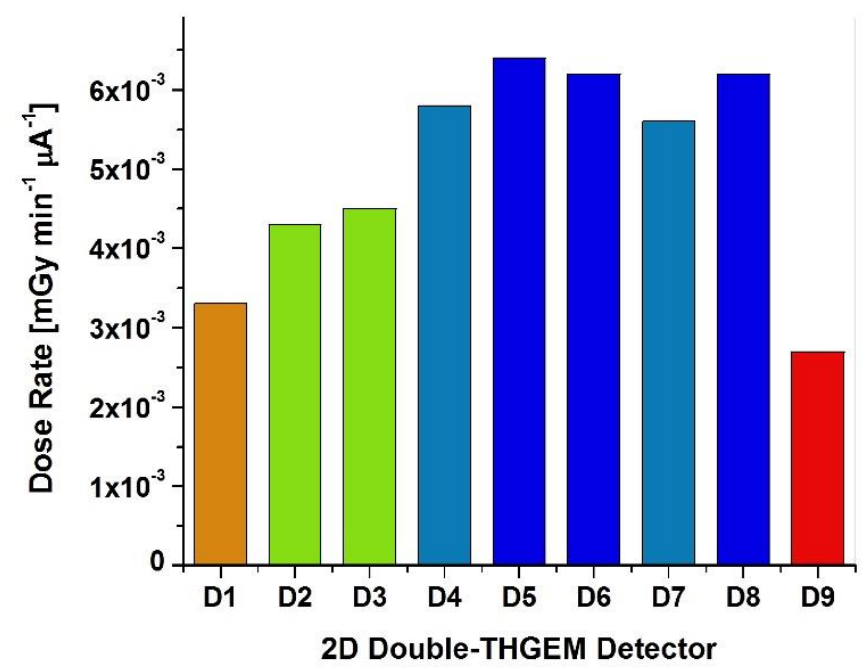

Fig. 7.52 The absorbed dose distribution inside the $6 \times 6 \mathrm{~cm}^{2}$ area of the $2 \mathrm{D}$ THGEM detector (an array of $3 \times 3$ gas cavity)

Featuring the spatial dose distribution over the detector area, the 2D THGEM detector has a unique beneficial advantage in contrast to the conventional TEPCs. This detector can overcome the adversity and tediousness of the neutron monitoring measurements, such as the study that have been carried out in chapter 6 , by measuring the spatial dose distribution in a single measurement. 


\subsection{D THGEM Detector with Smaller Size Sensitive Volume}

To investigate the minimum cavity size that can be employed with this kind of detector, two fabricated sensitive volumes of $2.5 \mathrm{~mm}$ and $1 \mathrm{~mm}$ diameter from Rexolite were used (see section 2.8). These measurements were carried out only for the central detector (D5) and the commercial ORTEC DSPEC with 16k ADC channels was used for data acquisition.

\section{$2.5 \mathrm{~mm}$ diameter gas cavity}

The good working THGEM \#5 from the new batch and the Rexolite layer consisting of an array of $3 \times 3$ gas cavities of $2.5 \mathrm{~mm}$ diameter are installed inside the same detector chamber. The detector was pumped down over night and filled with the TE propane gas at a pressure of 334 torr to simulate $2 \mu \mathrm{m}$ spherical tissue size. The same experimental methodology was employed. The only difference was the level of high voltage which had to be much higher as the gas pressure was about 2 times higher than for the $5 \mathrm{~mm}$ gas cavity. Therefore extra caution was necessary to avoid any sudden discharge due to the excessive applied high voltage. The first indication of the signal was at about $\mathrm{HV}_{\text {Cathode }}=$ $1460 \mathrm{~V}, \mathrm{HV}_{\mathrm{THGEM}}=1360 \mathrm{~V}$. At $\mathrm{HV}_{\text {Cathode }}=1560, \mathrm{HV}_{\mathrm{THGEM}}=1460$ the signal to noise ratio was extremely good for such a small size counter. The preamp and amplifier (gain of 25) signal observed on the oscilloscope is shown in Fig. $7.53 \mathrm{~d}$.

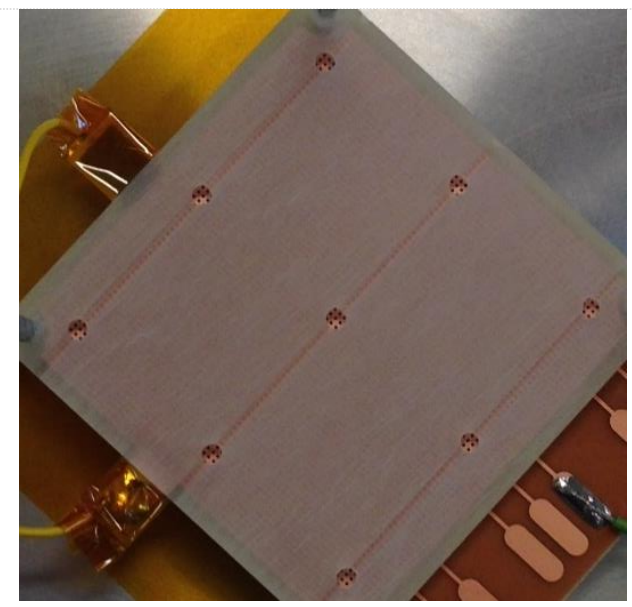

a.

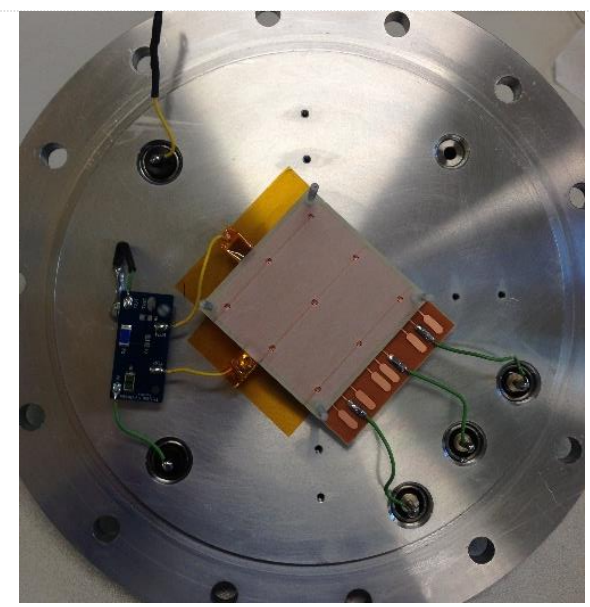

b. 


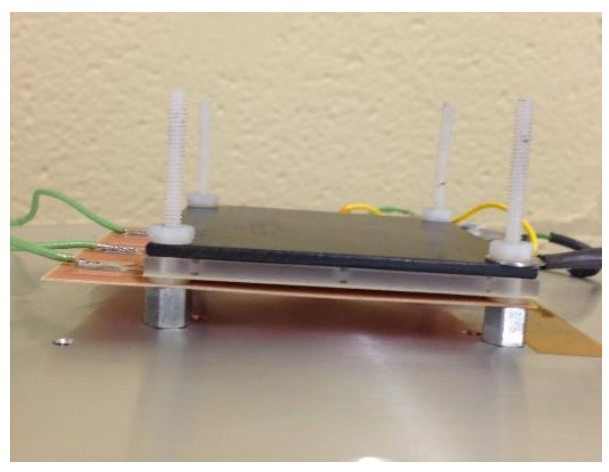

c.

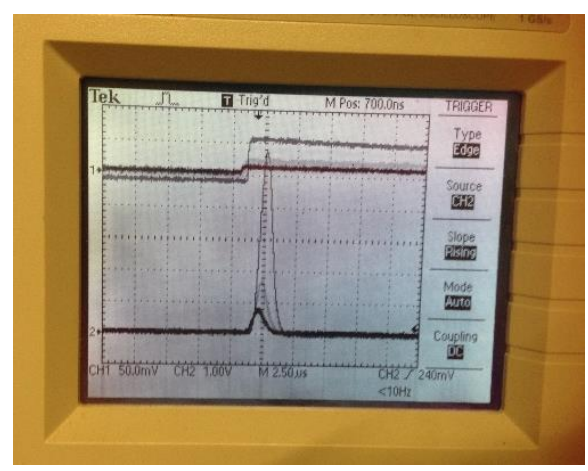

d.

Fig. 7.53 THGEM detector assembly with $2.5 \mathrm{~mm}$ diameter gas cavities a. a number of THGEM holes within the cavities, b. \& c. detector assembly, d. detector signal observed on the oscilloscope

The raw data at $\Delta \mathrm{V}_{\text {THGEM }}=1167 \mathrm{~V}$ are compared to $\Delta \mathrm{V}_{\text {THGEM }}=1200 \mathrm{~V}$ for $\mathrm{E}_{\mathrm{p}}=2.3$ $\mathrm{MeV}$ and $\mathrm{I}_{\mathrm{p}}=50 \mu \mathrm{A}$ and are shown in Fig. $7.54 a$. The data were acquired using the ORTEC DSPEC with the gain of 10 and 16k ADC channels. The count rate for the lower high voltage was $68 \mathrm{cps}$ and for the higher one was $164 \mathrm{cps}$. Fig. $7.54 \mathrm{~b}$ shows the detector response for two different proton energies 2.3 and $2.5 \mathrm{MeV}$. The event count rate for 2.3 $\mathrm{MeV}$ was $76 \mathrm{cps}$ and $160 \mathrm{cps}$ for the $2.5 \mathrm{MeV}$.

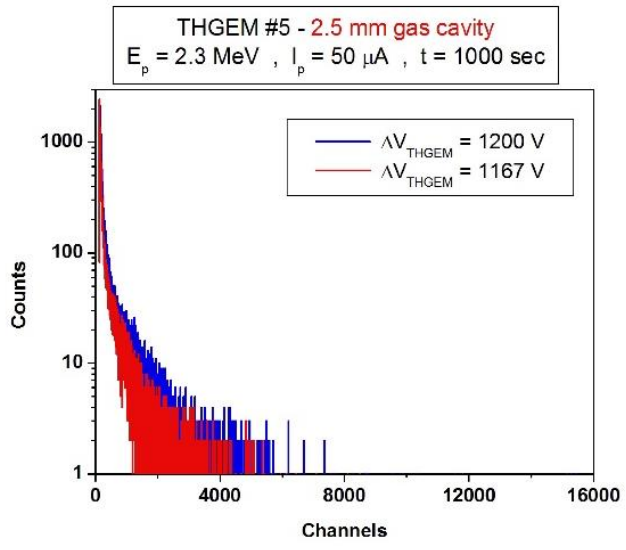

a.

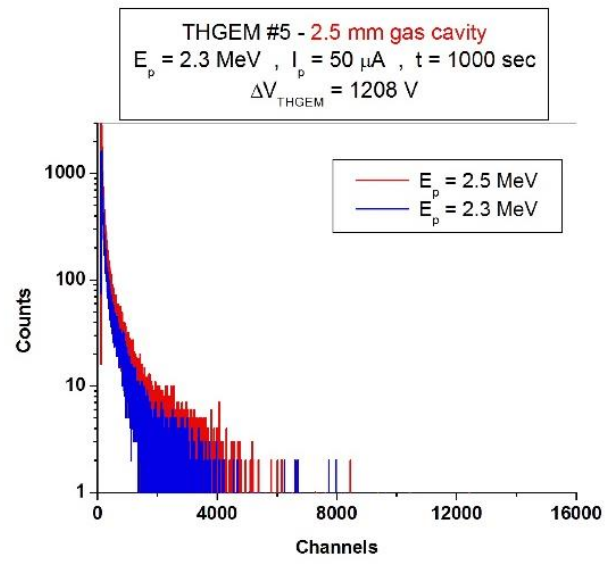

b. 


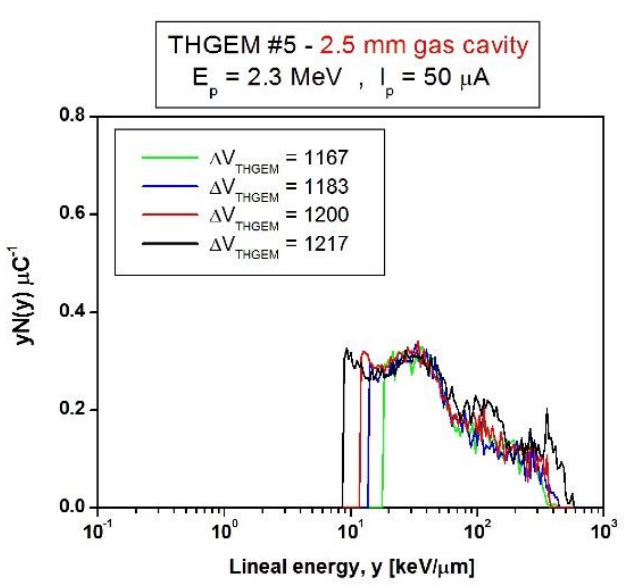

c.

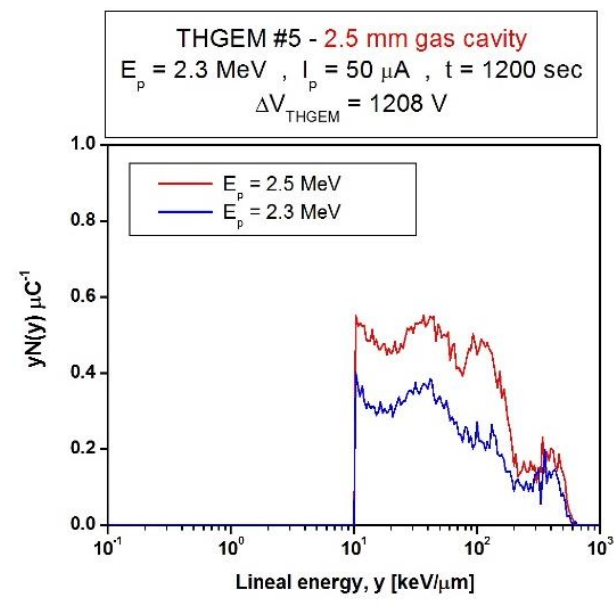

d.

Fig. 7.54 THGEM detector with $2.5 \mathrm{~mm}$ diameter gas cavities response for a. raw spectra for different applied high voltages across the THGEM, b. raw spectra for different proton energies, c. microdosimetric spectra for different applied high voltages across the THGEM, d. microdosimetric spectra for different proton energies

Even though the detector signal to noise ratio was very high, the microdosimetric spectra, shown in Fig. $7.54 c$ and $d$ did not have a consistent pattern compared to the case of $5 \mathrm{~mm}$ gas cavity size. There are multiple peaks and this made the lineal energy calibration harder as finding the right position of proton peak and edge is not feasible. Raising the high voltage up to this range did not have a significant effect on the spectra. However the decrease in the lower cut off end by increasing the high voltage was observed which is reasonable. The applied high voltage to the THGEM, which was set at $1217 \mathrm{~V}$, had to be slightly decreased to $\Delta \mathrm{V}_{\text {THGEM }}=1208 \mathrm{~V}$ as the first indication of spark was observed. There are two possibilities: 1) it seemed that due to the very small physical size of the detector there is a need for a much higher multiplication gain, so there is a need to raise high voltage even more, however the maximum achievable gain, and high voltage, was limited by the onset of discharge. Thus to overcome this issue, the use of doubleTHGEM arrangement is definitely beneficial and highly recommended. 2) As the radiation field was not strong enough, the detector was not efficient for a proton current of $50 \mu \mathrm{A}$. As shown before, it would be much more conclusive to use maximum achievable proton current (e.g $400 \mu \mathrm{A}$ for McMaster Tandetron accelerator facility), or test the detector in 
other intense fields. These two points have been investigated for a $1 \mathrm{~mm}$ diameter sensitive volume next.

\section{1 mm diameter gas cavity}

The double-THGEM arrangement was adopted for $1 \mathrm{~mm}$ diameter gas cavity as shown in Fig. 7.55 using two new THGEMs \#13 and \#14 which were never tested before.

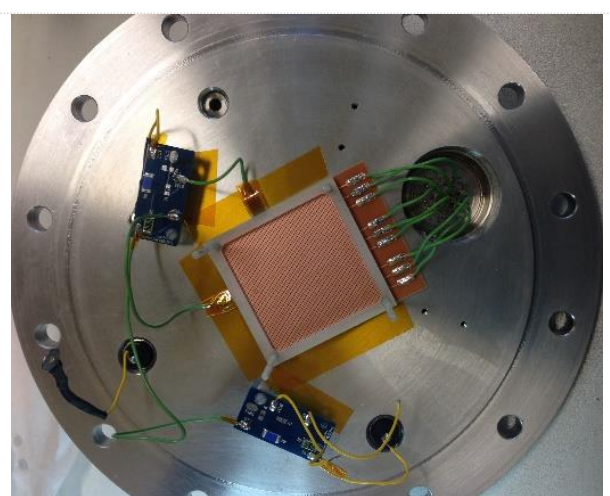

a.

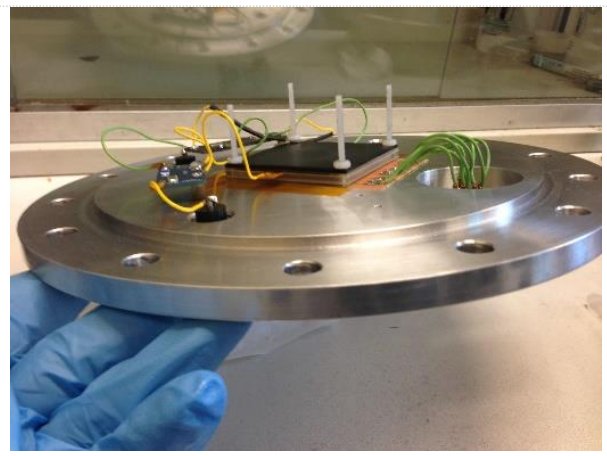

c.

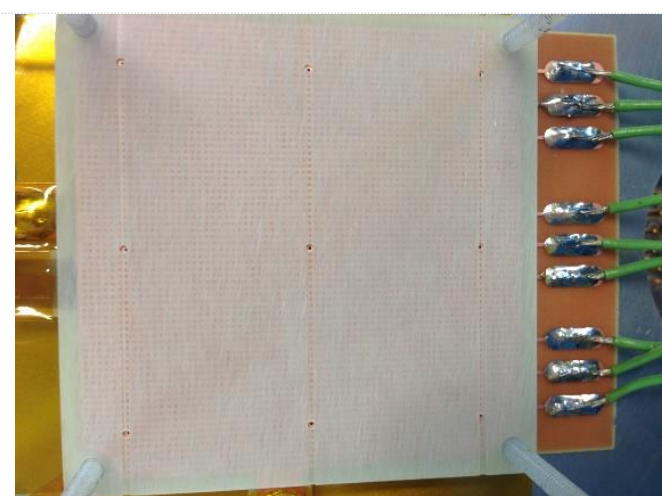

b.

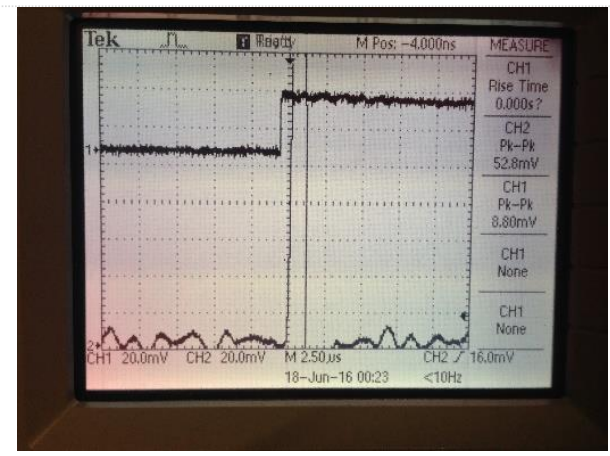

d.

Fig. 7.55 THGEM detector assembly with $1 \mathrm{~mm}$ diameter gas cavities, a. double-THGEM assembly using $2 \mathrm{~mm}$ square gap between THGEMs $\mathbf{b}$. a number of THGEM holes within the cavities, $\mathbf{c}$. detector assembly, d. preamp and amplifier (gain of 25) signals

The detector was pumped down over night and filled with the TE propane gas at a pressure of 834.4 torr. The measurement was carried out with $\mathrm{E}_{\mathrm{p}}=2.1 \mathrm{MeV}$ and $\mathrm{I}_{\mathrm{p}}=300$ $\mu \mathrm{A}$. It was more favourable to use higher proton energy for such a small size detector, however, the Tandetron accelerator could not reach to $2.3 \mathrm{MeV}$ or higher current for a 
while due to technical issues. As the gas pressure is 5 times and 2.5 times higher than in the case of $5 \mathrm{~mm}$ and $2.5 \mathrm{~mm}$ gas cavity sizes, respectively, the applied high voltage should be higher accordingly. On the other hand, as there was a double-THGEM configuration, the voltage across each THGEM was less and much bearable. The high voltage was raised gradually with extra caution and the detector signal was observed on the oscilloscope. Interestingly, the detector signal could reach to $32 \mathrm{mV}$ with an acceptable signal to noise ratio for the first time measurement with this small detector (see Fig. $7.55 d$ ).

The maximum safe high voltage that could be applied was $3200 \mathrm{~V}$ for the cathode and 3100 for THGEM. Thus the high voltage across each THGEM was $1292 \mathrm{~V}$. The count rate was $63 \mathrm{cps}$. The detector had a spark as the cathode and THGEM voltages were raised to $3600 \mathrm{~V}$ and $3500 \mathrm{~V}$ respectively. The raw and microdosimetric spectra are shown in Fig. 7.56. The lineal energy calibration was hard as there was not a specific proton peak there. There is a need for more gain for such a small detector, considering the fact that the radiation field was not intense.

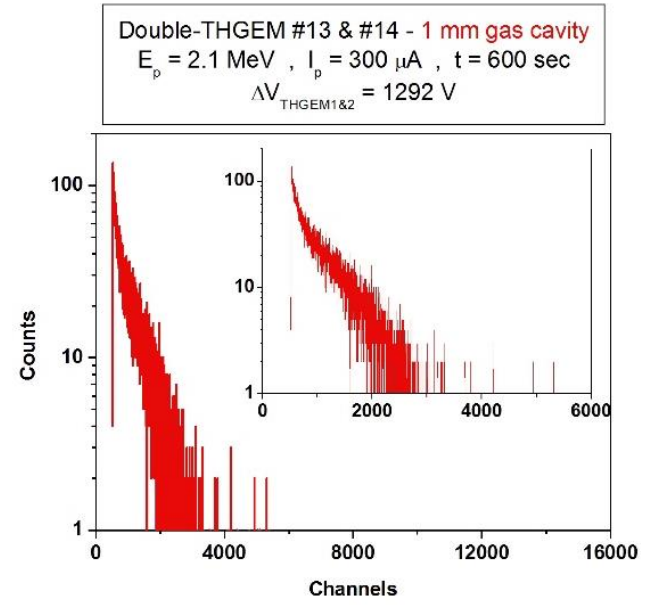

a.

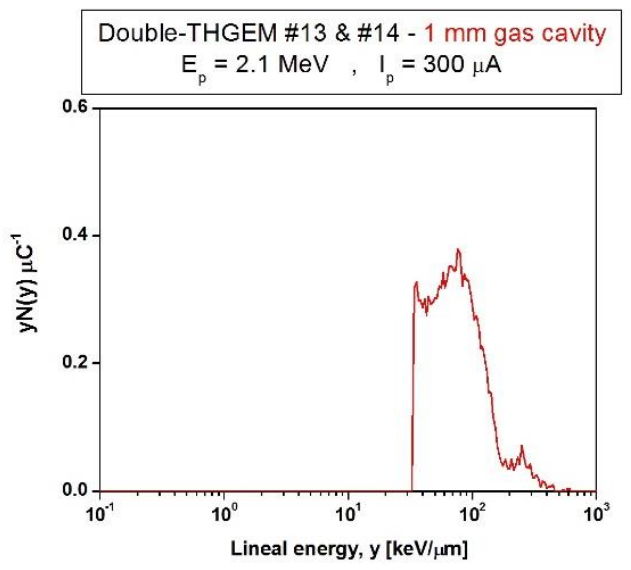

b.

Fig. 7.56 THGEM detector with $1 \mathrm{~mm}$ diameter gas cavity response a. raw spectrum, b. microdosimetric spectrum for $E_{p}=2.1 \mathrm{MeV}$ and $I_{p}=300 \mu \mathrm{A}$ 


\section{Chapter 8}

\section{Future Works and Conclusions}

8.1 Encountered Challenges and Proposed Future Works

8.2 Thesis Conclusion 


\subsection{Encountered Challenges and Proposed Future Works}

During the entire course of this research, the fact that an advanced detector had to be designed, constructed and implemented from scratch, many challenges were encountered that forced the author to gain prominent knowledge, skills, and experiences regarding using softwares, to design the detector layers, chamber and printed circuit boards. Also, the author obtained the student operator certificate for running Tandetron accelerator at McMaster University, which was used as a neutron source for her experimental measurements.

One of the major challenges was to maintain the same condition every time the detector was reassembled. In order to test the performance of each new THGEM the detector had to be opened and the new THGEM replaced. Extra care had to be taken in terms of cleaning the parts and the residues resulting from soldering. The chamber had to be sealed and pumped down and refilled with the TE gas at a specific pressure. Although it was always endeavoured to assemble the detector systematically and under the same condition, it was difficult to ensure that if all the variables were consistent from measurement to measurement. The gas pressure was the most challenging parameter. For the future work, it is proposed to add a pressure gauge to the design of the detector (installed on the chamber) so that the monitoring of the chamber pressure can be feasible over time. This would save much time on resolving some unexpected behaviours of the detector.

Another challenge was dealing with the THGEMs. As it was explained before many endeavours were made for improving the fabrication of the THGEMs. This does not mean that working with THGEM is difficult or time-consuming, but all the challenges are due to the technical fabrication issues by printed circuit board companies, who do not have a precise THGEM fabrication process as their routine like the group in CERN who are specialized for GEM/THGEM fabrication process. The preliminary negotiations have been done with Milplex for the amendments that can be done for the improvement of THGEMs. For the future works, it is worth to spend more time on the improvement of THGEMs and it is recommended to order them from various companies until the best quality is obtained. 
The neutron source was the other challenge that was encountered during the experimental process. First, the Tandetron accelerator at McMaster University which was the main neutron radiation source for this study was not always operating properly and there were periods that it was out of service for a while. This decelerated the progress of the research. Additionally, due to the technical matters and to save the Li target, it was not recommended to have the neutron beam on, for a longer period of time particularly for higher proton energies and currents. Due to the small size of 2D THGEM detector gas cavity, longer acquisition time (more than $1200 \mathrm{sec}$ ) was required for a better statistic fluctuations of the spectra. Therefore, it is recommended, if possible, to use a stronger neutron-gamma radiation field.

The second peak observed above the proton edge of the microdosimetric spectrum was the major challenge that has to be resolved thoroughly for the future works. Many attempts have been made to check all the factors that could affect this systematic issue. Improving the quality of THGEM fabrication process, keeping all the THGEMs inside a vacuum desiccator (Fisher Scientific Company) to prevent the expose of $\mathrm{Cu}$ surface to the air and handling them with extra care using gloves to avoid any moisture effects on the surface. Also, the THGEMs were blown with dry nitrogen gas to get rid of any debris and residues. This was the procedure that was employed and the best result of all was obtained with THGEM \#5 from the new batch. The microdosimetric response was extremely consistent and comparable with the response of the standard spherical 1/2" TEPC at the same position inside the irradiation cavity. This THGEM detector results reproduced from 4 times measurements over a month. However, once the detector was opened and reassembled to replace the $5 \mathrm{~mm}$ sensitive volumes with $2.5 \mathrm{~mm}$ sensitive volume, the increase of the high voltage due to the higher cavity gas pressure led to the breakdown of the THGEM. Although the same systematic assembly procedure was used, this result with one proton peak and sharp edge have not been reproduced ever after with any other THGEMs. Obviously, this requires further exploration. Therefore it is proposed to change the THGEM design with less surface $\mathrm{Cu}$. The THGEM holes that cover the area of each 9 detectors will be kept and the rest of the holes have to be omitted. This has two advantages: 
1) less surface area lead to less $\mathrm{Cu}$ activation when the detector is irradiated with a neutron beam, 2) This may help the printed circuit board manufacturer as they need to drill much fewer holes across the entire area of the THGEM. Hence the quality of THGEM holes can be improved considerably. In addition, the fabrication of bigger size THGEM detectors in terms of the area (like an array of $10 \times 10, \ldots$ ) would be more feasible and much more cost effective.

As it was mentioned before, it is believed that the activation of $\mathrm{Cu}$ inside the detector can cause a discrepancy of the lower lineal energy region of the spectrum between THGEM detector and standard 1/2" TEPC. Thus to confirm this, for the future work it is worth monitoring the activity of $\mathrm{Cu}$ on the THGEM and readout board layers immediately after a long measurement. For instance, at McMaster irradiation facility the $4 \pi$ arrangement of $9 \mathrm{NaI}(\mathrm{Tl})$ detection system (in the Tandetron accelerator control room) is the closest gamma detection system, which can be used for the gamma ray signal to be counted. However, extra care has to be taken in comply with the health physics safety rules and regulations for opening the detector when it is activated.

As it was discussed previously, the physical size of the detector (cavity dimension) is indeed the main controlling parameter in reducing the adverse effects of pulse pileup and therefore dead time occurring in high-intensity radiation fields. Hence it is desirable and beneficial to spend more time to investigate the smaller size detector gas cavity. For future work, it is proposed to employ the multiple-THGEM arrangement to increase the gain considerably without applying a large voltage across each THGEM individually.

Additional future works might include optimization of the MMI-DSP system for a more user-friendly interface that can give the users real-time options that now can be obtained with DSPEC. For instance, it would be much more helpful in terms of understanding the detector response spectrum if MMI-DSP allowed the user to have the spectrum characterization at the time of measurement. Also, the pulser calibration was much more time consuming with MMI-DSP compare to DSPEC, as the user had to save the pulser spectrum at the time of measurement and analyze and fit each peak one by one to get the pulser calibration. As there were 9 detectors involved in each measurement for this study 
(and will be much more in future), it was extremely tedious to fit at least 10 pulser peaks for each detector and then calculate the 9 calibration lines. This burden was resolved by writing a code in MATLAB to do the peak fits and calibration lines. However, it will be much more favorable to add this option to the MMI-DSP programming code itself for the optimization phase of the system.

\subsection{Thesis Conclusion}

An advanced 2D THGEM TEPC was successfully designed, constructed and tested using a mixed neutron and gamma-rays radiation field at McMaster University. In parallel, a multi-input digital pulse processing system, which was designed and constructed specifically for this project (by Kenrick Chin) has been effectively tested and employed throughout this study to process all the signal outputs from the 9 detectors individually and simultaneously. The prototype system was tested using a $\mathrm{NaI}(\mathrm{Tl})$ detector in comparison with a traditional analogue system and a commercial digital system and the results of the comprehensive benchmark tests were shown that its performance is comparable to the conventional signal processors.

From the results presented in this thesis it is evident that even though the THGEM detector size ( $5 \mathrm{~mm}$ gas cavity) is about 2.54 times smaller than the 1/2" TEPC and about 11.38 times smaller than the 2" TEPC, whose efficiencies are much higher, the THGEM detector showed a consistent microdosimetric pattern. The measured mean value of the absorbed dose rate for the central THGEM detector (D5) was measured to be $0.00613 \pm$ $0.00061\left[\mathrm{mGy} \mathrm{min}^{-1} \mu \mathrm{A}^{-1}\right]$. Also, the responses of all the other 8 detectors were extensively investigated, compared to the central detector and the absorbed dose rates distribution was obtained for the positions of $3 \times 3$ array of detectors.

The detector performance was also examined using double THGEMs. The doubleTHGEM arrangement was favoured over a single THGEM as it permits an increase in the gain of the signal without undue high voltage stress on the THGEM.

The detectors with 2 times and 5 times smaller sensitive volumes were fabricated and tested and the preliminary test results were promising and showed the potential for further development. 
Likewise, the ability to confidently employ the McMaster custom made signal processing system with the 2D THGEM detector was ascertained. The microdosimetric spectra from the new system were consistent with those extracted from the conventional system for various fields. However, there were some limitations associated with the prototype system which has to be resolved for the future works.

This study proved that the 2D TEPC based on the THGEM technology, together with the cost effective custom made multi-input digital signal processing system can be used as a promising detector for measuring the absorbed dose rate distribution over an area. It can be concluded that this small cavity counter opens new possibilities in applications for highintensity radiation fields as well as in nanodosimetry (simulate smaller tissue sizes). It would be our future goal to further optimize the detector with a larger area and for a larger number of detector arrays to develop an accurate versatile tool that can be used confidently in measuring dose distribution in any mixed radiation fields. 


\section{REFERENCES}

Aslam, Prestwich, W.V., McNeill, F.E. and Waker, A.J., 2003. Investigating the TEPC radiation quality factor response for low energy accelerator based clinical applications. Radiation protection dosimetry, 103(4), pp.311-322.

Badhwar, G.D., Robbins, D.E., Gibbons, F. and Braby, L.A., 2002. Response of a tissue equivalent proportional counter to neutrons. Radiation measurements, 35(6), pp.551-556.

Benjamin, P.W., Kemshall, C.D. and Redfearn, J., 1968. A high resolution spherical proportional counter. Nuclear Instruments and Methods, 59(1), pp.77-85.

Bhatia, C. et al., 2015. A neutron activation technique for manganese measurements in humans. Journal of Trace Elements in Medicine and Biology, 31, pp.204-208.

Booz, J., 1984. Advantages of introducing microdosimetric instruments and methods into radiation protection. Radiation Protection Dosimetry, 9(3), pp.175-183.

Bordy, J.M., Barthe, J., Lahaye, T., Boutruche, B. and Segur, P., 1995. Improving a multicellular tissueequivalent proportional counter for personal neutron dosimetry. Radiation Protection Dosimetry, 61(1-3), pp.175-178.

Boutruche, B., Bordy, J.M., Barthe, J., Segur, P. and Portal, G., 1994. A new concept of a high sensitivity tissue-equivalent proportional counter for individual neutron dosimetry. Radiation Protection Dosimetry, 52(1-4), pp.335-338.

Braby, L.A., 2015. Experimental microdosimetry: history, applications and recent technical advances. Radiation protection dosimetry, p.ncv137.

Bramlitt, E.T. and Shonka, J.J., 2015. Radiation exposure of aviation crewmembers and cancer. Health physics, 108(1), pp.76-86.

Breskin, A., Alon, R., Cortesi, M., Chechik, R., Miyamoto, J., Dangendorf, V., Maia, J.M. and Dos Santos, J.M.F., 2009. A concise review on THGEM detectors. Nuclear Instruments and Methods in Physics Research Section A: Accelerators, Spectrometers, Detectors and Associated Equipment, 598(1), pp.107-111.

Breskin, A., Cortesi, M., Alon, R., Miyamoto, J., Peskov, V., Bartesaghi, G., Chechik, R., Dangendorf, V., Maia, J.M. and dos Santos, J.M.F., 2010. The THGEM: A thick robust gaseous electron multiplier for radiation detectors.Nuclear Instruments and Methods in Physics Research Section A: Accelerators, Spectrometers, Detectors and Associated Equipment, 623(1), pp.132-134.

Burigo, L., Pshenichnov, I., Mishustin, I. and Bleicher, M., 2014. Microdosimetry spectra and RBE of $1 \mathrm{H}$, ${ }^{4} \mathrm{He},{ }^{7} \mathrm{Li}$ and ${ }^{12} \mathrm{C}$ nuclei in water studied with Geant4. Nuclear Instruments and Methods in Physics Research Section B: Beam Interactions with Materials and Atoms, 320, pp.89-99.

Burmeister, J., Kota, C. and Maughan, R.L., 1999. Paired miniature tissue-equivalent proportional counters for dosimetry in high flux epithermal neutron capture therapy beams. Nuclear Instruments and Methods in Physics Research Section A: Accelerators, Spectrometers, Detectors and Associated Equipment, 422(1), pp.606-610.

Burmeister, J., Kota, C., Maughan, R.L. and Waker, A.J., 2001. Miniature tissue-equivalent proportional counters for BNCT and BNCEFNT dosimetry.Medical physics, 28(9), pp.1911-1925.

Burmeister, J., Kota, C., Maughan, R.L. and Waker, A.J., 2002. Characterization of miniature tissueequivalent proportional counters for neutron radiotherapy applications. Physics in medicine and biology, 47(10), p.1633.

Byun, S.H. et al., 2007. Dosimetric characterization of the irradiation cavity for accelerator-based in vivo neutron activation analysis. Physics in medicine and biology, 52(6), pp.1693-1703. 
Byun, S.H. et al., 2009. Simulation and First Test of a Microdosimetric Detector Based on a Thick Gas Electron Multiplier. IEEE Transactions on Nuclear Science, 56(3), pp.1108-1113.

Chechik, R., Cortesi, M., Breskin, A., Vartsky, D., Bar, D. and Dangendorf, V., 2006. Thick GEM-like (THGEM) detectors and their possible applications. arXiv preprint physics/0606162.

Cortesi, M., Chechik, R., Breskin, A., Guedes, G., Dangendorf, V., Vartzky, D. and Bar, D., 2007. Advances in imaging THGEM-based detectors.Nuclear Instruments and Methods in Physics Research Section A: Accelerators, Spectrometers, Detectors and Associated Equipment, 572(1), pp.175-176.

Darvish-Molla, S., Prestwich, W. V \& Byun, S.H., 2015. A comprehensive radiation dose measurements and Monte Carlo simulation for the ${ }^{7} \mathrm{Li}(\mathrm{p}, \mathrm{n})$ accelerator neutron field. Radiation Protection Dosimetry. DOI: $10.1093 / \mathrm{rpd} / \mathrm{ncv} 428$

De Nardo, L., Cesari, V., Donà, G., Magrin, G., Colautti, P., Conte, V. and Tornielli, G., 2004. MiniTEPCs for radiation therapy. Radiation protection dosimetry, 108(4), pp.345-352.

De Oliveira, R. and Pinto, S.D., CERN—European Organization for Nuclear Research, 2013. Method of manufacturing a gas electron multiplier. U.S. Patent 8,597,490.

Farahmand, M., Bos, a. J.J. \& Van Eijk, C.W.E., 2003. Gas electron multiplier (GEM) operation with tissue-equivalent gases at various pressures. Nuclear Instruments and Methods in Physics Research, Section A: Accelerators, Spectrometers, Detectors and Associated Equipment, 506(1-2), pp.160-165.

Farahmand, M. \& Nardo, L. De, 2015. Microdosimetric measurements of a tissue-equivalent proportional counter based on a gas electron multiplier down to $140 \mathrm{~nm}$ simulated site sizes, pp.1-9.

Gerdung, S., Pihet, P., Grindborg, J.E., Roos, H., Schrewe, U.J. and Schuhmacher, H., 1995. Operation and application of tissue equivalent proportional counters. Radiation protection dosimetry, 61(4), pp.381-404.

Goodhead, D.T., 1987. Relationship of microdosimetric techniques to applications in biological systems. The dosimetry of ionizing radiation, 2, pp.1-89. ACADEMIC PRESS. INC., USA

Goodhead, D.T., 1982. An assessment of the role of microdosimetry in radiobiology. Radiation Research, 91(1), pp.45-76.

Hanu, a., Byun, S.H. \& Prestwich, W. V., 2010. A Monte Carlo simulation of the microdosimetric response for thick gas electron multiplier. Nuclear Instruments and Methods in Physics Research, Section A: Accelerators, Spectrometers, Detectors and Associated Equipment, 622(1), pp.270-275.

Hanu, a. 2013. Development of a Thick Gaseous Electron Multiplier Imaging Detector using a TwoDimensional Delay Line Readout. PhD Thesis. McMaster University

Hanu, a. R., Prestwich, W.V. \& Byun, S.H., 2015. A data acquisition system for two-dimensional position sensitive micropattern gas detectors with delay-line readout. Nuclear Instruments and Methods in Physics Research Section A: Accelerators, Spectrometers, Detectors and Associated Equipment, 780, pp.33-39.

ICRP. 1991. 1990 Recommendations of the International Commission on Radiological Protection. ICRP

Publication 60. Annals of the ICRP 21 (1-3)

ICRU. 1970b. Linear Energy Transfer. ICRU report 16, Bethesda, Maryland

ICRU. 1980. Radiation Quantities and Units. ICRU report 33, Bethesda, Maryland

ICRU. 1983. Microdosimetry. ICRU report 36, Bethesda, Maryland

ICRU. 1986. The quality factor in radiation protection. ICRU report 40, Bethesda, Maryland

ICRU. 1993. Stopping Power and Ranges for Protons and Alpha Particles. ICRU report 49, Bethesda, Maryland

Kellerer, A.M., 1985. Fundamentals of microdosimetry. The dosimetry of ionizing radiation, 1, pp.77-162. ACADEMIC PRESS. INC., USA 
Kellerer, A.M. and Rossi, H.H., 1972. Curr. Topics Radiat. Res. Q, 8(85), p.1978.

Kellerer, A.M. and Rossi, H.H., 1978. A generalized formulation of dual radiation action. Radiation Research, 75(3), pp.471-488.

Kellerer, A.M., 1981. Concepts of geometrical probability relevant to microdosimetry and dosimetry. $7^{\text {th }}$ Symposium on Microdosimetry. Oxford, UK, pp. 1049-1062

Kliauga, P., Waker, A.J. and Barthe, J., 1995. Design of tissue-equivalent proportional counters. Radiation Protection dosimetry, 61(4), pp.309-322.

Kliauga, P., Rossi, H.H. and Johnson, G., 1989. A multi-element proportional counter for radiation protection measurements. Health physics, 57(4), pp.631-636.

Kliauga, P., Onizuka, Y. and Magrin, G., 1996. Microdosimetric analysis of radiation from a clinical mammography

machine using realistic breast phantoms and a miniature proportional counter. Physics in medicine and biology, 41(11), p.2295.

Knoll, G.F., 2010. Radiation detection and measurement. John Wiley \& Sons.

Kowalski, T.Z., 2015. Microdosimetric response of proportional counters filled with different tissue equivalent gas mixtures. Journal of Instrumentation, 10(3).

Lee, C. \& Zhou, X., 1999. Thick target neutron yields for the ${ }^{7} \mathrm{Li}(\mathrm{p}, \mathrm{n}){ }^{7}$ Be reaction near threshold. Nuclear Instruments and Methods in Physics Research ..., 152, pp.1-11.

Maia, J.M., Mörmann, D., Breskin, A., Chechik, R., Veloso, J.F.C.A. and dos Santos, J.M.F., 2007. SingleUV-photon 2-D imaging with multi-GEM detectors. Nuclear Instruments and Methods in Physics Research Section A: Accelerators, Spectrometers, Detectors and Associated Equipment, 580(1), pp.373-376.

Marchiori, E., 2014. Development of a multiple microdosimetric detector based on GEM (Gas Electron Multiplier), for hadrontherapy applications.

Matysiak, W., Prestwich, W. V. \& Byun, S.H., 2008. Measurement of the thick target 7Li(p,n) neutron source spectrum using a $3 \mathrm{He}$ ionization chamber. Nuclear Instruments and Methods in Physics Research, Section A: Accelerators, Spectrometers, Detectors and Associated Equipment, 592(3), pp.316-324.

Matysiak, W. et al., 2013. In-Vivo Neutron Activation Analysis for Aluminium in Bone: System Upgrade and Improve Data Analysis. AECL Nuclear Review, 2(2), pp.27-32.

Ohshita, H. et al., 2010. Development of a neutron detector with a GEM. Nuclear Instruments and Methods in Physics Research Section A: Accelerators, Spectrometers, Detectors and Associated Equipment, 623(1), pp.126-128.

Orchard, G.M. 2010. Development of a thick gas electron multiplier for microdosimetry. PhD Thesis. McMaster University

Orchard, G.M. et al., 2011. Development of a thick gas electron multiplier for microdosimetry. Nuclear Instruments and Methods in Physics Research Section A: Accelerators, Spectrometers, Detectors and Associated Equipment, 638(1), pp.122-126.

Ostling, J., 2006. New Efficient Detector for Radiation Therapy Imaging using Gas Electron Multipliers. PhD Thesis. Karolinska Institutet \& Stockholm University

Pejović-Milić, A. et al., 2006. Development of an irradiation/shielding cavity for in vivo neutron activation analysis of manganese in human bone. Journal of Radioanalytical and Nuclear Chemistry, 269(2), pp.417-420.

Periale, L., Peskov, V., Carlson, P., Francke, T., Pavlopoulos, P., Picchi, P. and Pietropaolo, F., 2002. Detection of the primary scintillation light from dense $\mathrm{Ar}, \mathrm{Kr}$ and Xe with novel photosensitive gaseous detectors. Nuclear Instruments and Methods in Physics Research Section A: Accelerators, Spectrometers, Detectors and Associated Equipment, 478(1), pp.377-383. 
Periale, L., Peskov, V., Carlson, P., Iacobeause, C., Francke, T., Pavlopoulos, N., Pietropaolo, F., Sokolova, T. and Picchi, P., 2003. Evaluation of various planar gaseous detectors with CsI photocathodes for the detection of primary scintillation light from noble gases. Nuclear Instruments and Methods in Physics Research Section A: Accelerators, Spectrometers, Detectors and Associated Equipment, 497(1), pp.242-248.

Peskov, V., Baibussinov, B., Centro, S., Di Mauro, A., Lund-Jensen, B., Martinengo, P., Nappi, E.,

Oliveira, R., Pietropaolo, F., Picchi, P. and Periale, L., 2007. Development and first tests of GEM-like detectors with resistive electrodes. IEEE Transactions on Nuclear Science, 54(5), pp.1784-1791.

Qashua, N. 2010. The effect of high dose rate on tissue equivalent proportional counter measurement in mixed neutron-gamma fields. MSc. Thesis. University of Ontario Institute of Technology.

Rollet, S., Colautti, P., Grosswendt, B., Herault, J., Wind, M., Gargioni, E., Beck, P., Latocha, M. and Moro, D., 2011.Microdosimetric assessment of the radiation quality of a therapeutic proton beam: comparison between numerical simulation and experimental measurements. Radiation protection dosimetry, 143(2-4) pp.

Rossi, H.H. and Rosenzweig, W., 1955. Measurements of neutron dose as a function of linear energy transfer. Radiation research, 2(5), pp.417-425.

Rossi, H.H. and Failla, G., 1956. Tissue-equivalent ionization chambers. Nucleonics (US), 14(2).

Santa Cruz, G.A.S. and Zamenhof, R.G., 2004. The microdosimetry of the 10B reaction in boron neutron capture therapy: a new generalized theory.Radiation research, 162(6), pp.702-710.

Santa Cruz, G.A., 2016. Microdosimetry: Principles and applications. Reports of Practical Oncology \& Radiotherapy, 21, pp. 135-139

Sauli, F., 1997. GEM: A new concept for electron amplification in gas detectors. Nuclear Instruments and Methods in Physics Research Section A: Accelerators, Spectrometers, Detectors and Associated Equipment, 386(2), pp.531-534.

Sauli, F., 2003. Development and applications of gas electron multiplier detectors. Nuclear Instruments and Methods in Physics Research Section A: Accelerators, Spectrometers, Detectors and Associated Equipment, 505(1), pp.195-198.

Seydaliev, M. 2007. Development and Test of a GEM-Based TEPC for Neutron Protection Dosimetry. PhD Thesis. Georgia Institute of Technology

Shalem, C., Chechik, R., Breskin, A. and Michaeli, K., 2006. Advances in Thick GEM-like gaseous electron multipliers-Part I: atmospheric pressure operation. Nuclear Instruments and Methods in Physics Research Section A: Accelerators, Spectrometers, Detectors and Associated Equipment, 558(2), pp.475-489.

Shalem, C.K., Chechik, R., Breskin, A., Michaeli, K. and Ben-Haim, N., 2006. Advances in thick GEMlike gaseous electron multipliers Part II: Low-pressure operation. Nuclear Instruments and Methods in Physics Research Section A: Accelerators, Spectrometers, Detectors and Associated Equipment, 558(2), pp.468-474.

Smathers, J.B., Otte, V.A., Smith, A.R., Almond, P.R., Attix, F.H., Spokas, J.J., Quam, W.M. and Goodman, L.J., 1977. Composition of A-150 tissue-equivalent plastic. Medical physics, 4(1), pp.74-77.

Srdoč, D., 1970. Experimental technique of measurement of microscopic energy distribution in irradiated matter using Rossi counters. Radiation research, 43(2), pp.302-319.

Spirou, G.M., Byun, S.H. \& Prestwich, W. V., 2007. Comparison of three pulse processing systems for microdosimetry. IEEE Nuclear Science Symposium Conference Record, 2(5), pp.1649-1652.

Uno, S. et al., 2012. Two-dimensional Neutron Detector with GEM and its Applications. Physics Procedia, 26, pp.142-152 
Van Eijk, C.W.E., 2004. Neutron detection and neutron dosimetry. Radiation protection dosimetry, 110 (14), pp.5-13.

Veenhof, R., 1998. GARFIELD, recent developments. Nuclear Instruments and Methods in Physics Research, Section A: Accelerators, Spectrometers, Detectors and Associated Equipment, 419(2-3), pp.726-730

Waker, A.J., 1985. Experimental uncertainties in microdosimetric measurements and an examination of the performance of three commercially produced proportional counters. Nuclear Instruments and Methods in Physics Research Section A: Accelerators, Spectrometers, Detectors and Associated Equipment, 234(2), pp.354-360.

Waker, A.J. and Marco, M., 1992. The application of microdosimetry to the metrology of low-energy X rays used in mammography. The British journal of radiology, 65(771), pp.258-261.

Waker, A.J., 1995. Principals of experimental microdosimetry. Radiation protection dosimetry, 61(4), pp.297-308.

Waker, A.J., Schrewe, U., Burmeister, J., Dubeau, J. and Surette, R.A., 2002. Classical microdosimetry in radiation protection dosimetry and monitoring. Radiation protection dosimetry, 99(1-4), pp.311316.

Waker, A.J., 2010. A preliminary study of the performance of a novel design of multi-element tissue equivalent proportional counter for neutron monitoring. Radiation Measurements, 45(10), pp.13091312.

Waker, A.J. and Lori, J., 2011. Design of a multi-element TEPC for neutron monitoring. Radiation protection dosimetry, 143(2-4), pp.463-466.

Wambersie, A., Pihet, P. and Menzel, H.G., 1990. The role of microdosimetry in radiotherapy. Radiation Protection Dosimetry, 31(1-4), pp.421-432.

Wang, C.-K.C., Seidaliev, M. \& Mandapaka, A., 2008. Development and test of a GEM-based TEPC for neutron protection dosimetry. Health physics, 94(5), pp.440-448

Wuu, C.S., Amols, H.I., Kliauga, P., Reinstein, L.E. and Saraf, S., 1992. Microdosimetry for boron neutron capture therapy. Radiation research, 130(3), pp.355-359.

Zaider, M., Rossi, B.H.H. and Zaider, M., 1996. Microdosimetry and its Applications. Springer-Verlag Berlin, Heidelberg, Germany

Zhang, W., Wang, Z., Liu, Y., Li, C., Xiao, X., Luo, H., Chen, J. and Li, W., 2014. TEPC performance for a reference standard. Radiation protection dosimetry, 158(2), pp.246-250. 